\title{
RENORMALIZATION GROUP AND PHASE TRANSITIONS IN SPIN,
} GAUGE, AND QCD LIKE THEORIES

\author{
by \\ Yuzhi Liu
}

A thesis submitted in partial fulfillment of the requirements for the Doctor of Philosophy

degree in Physics in the
Graduate College of The
University of Iowa

August 2013

Thesis Supervisor: Professor Yannick Meurice 
Copyright by

YUZHI LIU

2013

All Rights Reserved 
Graduate College

The University of Iowa

Iowa City, Iowa

CERTIFICATE OF APPROVAL

PH.D. THESIS

This is to certify that the Ph.D. thesis of

\section{Yuzhi Liu}

has been approved by the Examining Committee for the thesis requirement for the Doctor of Philosophy degree in Physics at the August 2013 graduation.

Thesis Committee:

Yannick Meurice, Thesis Supervisor

Andreas S. Kronfeld

Vincent G. J. Rodgers

Wayne N. Polyzou

Craig Pryor 
To the memory of my father, Deliang Liu. 


\section{ACKNOWLEDGMENTS}

Firstly, I would like to thank sincerely my advisor Professor Yannick Meurice for his guidance through the period of my work at University of Iowa. His consistent encouragement and stimulation is invaluable to me. Sharing his creative way of doing physics is one of the most precious experiences I have had at Iowa. Without his extensive discussion and guidance during every stage of the work, this thesis would never have been possible to accomplish.

I am also grateful to Dr. Andreas Kronfeld for helpful discussions and guidance during my one year stay at Fermilab. He has greatly broadened my knowledge on lattice gauge theories and especially lattice flavor physics in QCD. Dr. Ruth Van de Water played an important role on initiating my project at Fermilab and gave me guidance on how the project should proceed.

I thank Professor Anna Hasenfratz for teaching me Monte Carlo renormalization group and two lattice matching methods. I thank Dr. Zhiyuan Xie and Professor Tao Xiang for teaching me tensor renormalization group concepts and technique during my stay at KITPC.

I would like to thank all the professors who have taught me physics and helped me in various occasions, including M. E. Flatté, William H. Klink, Wayne Polyzou, Craig Pryor, Mary-Hall Reno, Vincent Rodgers, and John Schweitzer. I also wish to thank Deborah Foreman, Heather Mineart, Jeanne Mullen and other staff members in the Department for their administrative assistance and help.

Many thanks to my friends and fellow graduate students at the Department, especially to Alan denBleyker, Daping Du, Yan Feng, Ran Lin, Zhenzhen Wang, Shengyi Ye, and Haiyuan Zou. Their friendship have made my life at Iowa a joyful experience. 
Special thanks go to _ who have made my effort more meaningful. I am grateful to my mother for her consistent support and understanding. 


\begin{abstract}
In this thesis, we study several different renormalization group (RG) methods, including the conventional Wilson renormalization group, Monte Carlo renormalization group (MCRG), exact renormalization group (ERG, or sometimes called functional RG), and tensor renormalization group (TRG).

We use the two dimensional nearest neighbor Ising model to introduce many conventional yet important concepts. We then generalize the model to Dyson's hierarchical model (HM), which has rich phase properties depending on the strength of the interaction. The partition function zeros (Fisher zeros) of the HM model in the complex temperature plane is calculated and their connection with the complex RG flows is discussed. The two lattice matching method is used to construct both the complex RG flows and calculate the discrete $\beta$ functions. The motivation of calculating the discrete $\beta$ functions for various HM models is to test the matching method and to show how physically relevant fixed points emerge from the complex domain.
\end{abstract}

We notice that the critical exponents calculated from the HM depend on the blocking parameter $b$. This motivated us to analyze the connection between the discrete and continuous RG transformation. We demonstrate numerical calculations of the ERG equations. We discuss the relation between Litim and Wilson-Polchinski equation and the effect of the cut-off functions in the ERG calculation.

We then apply methods developed in the spin models to more complicated and more physically relevant lattice gauge theories and lattice quantum chromodynamics (QCD) like theories. Finite size scaling (FSS) technique is used to analyze the Binder cumulant of the $S U(2)$ lattice gauge model. We calculate the critical exponent $\nu$ and $\omega$ of the model and show that it is in the same universality class as 
the three dimensional Ising model.

Motivated by the walking technicolor theory, we study the strongly coupled gauge theories with conformal or near conformal properties. We compare the distribution of Fisher zeros for lattice gauge models with four and twelve light fermion flavors. We also briefly discuss the scaling of the zeros and its connection with the infrared fixed point (IRFP) and the mass anomalous dimension.

Conventional numerical simulations suffer from the critical slowing down at the critical region, which prevents one from simulating large system. In order to reach the continuum limit in the lattice gauge theories, one needs either large volume or clever extrapolations. TRG is a new computational method that may calculate exponentially large system and works well even at the critical region. We formulate the TRG blocking procedure for the two dimensional $O(2)$ (or $X Y$ ) and $O(3)$ spin models and discuss possible applications and generalizations of the method to other spin and lattice gauge models.

We start the thesis with the introduction and historical background of the RG in general. 


\section{TABLE OF CONTENTS}

LIST OF TABLES ........................ . . ix

LIST OF FIGURES . . . . . . . . . . . . . . . . . . . . xi

\section{CHAPTER}

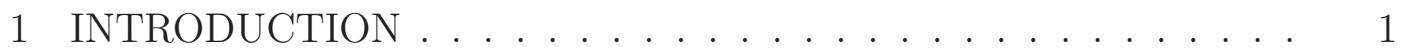

1.1 Introduction . . . . . . . . . . . . . . . . 1

1.1.1 Spin Models . . . . . . . . . . . . . . . . . . 6

1.1.2 Gauge Models . . . . . . . . . . . . . . . . 7

1.2 Research Results . . . . . . . . . . . . . . . . . . 9 9

2 RENORMALIZATION GROUP METHODS ON SPIN MODELS IN THE HIERARCHICAL APPROXIMATION . . . . . . . . . 12

2.1 Ising Model . . . . . . . . . . . . . . . . . . . . . . . . 12

2.2 Dyson's Hierarchical Model . . . . . . . . . . . . . . . . . . 15

2.2.1 Definition Of The Model . . . . . . . . . . . . . . 16

2.2.2 Partition Function Of The hierarchical Model . . . . . . 19

2.2.3 Extension Of The Recursion Formula . . . . . . . . . . 20

2.2.4 Zeros Of The Partition Function . . . . . . . . . . . 21

2.2.5 Distribution Of The Zeros For Different Variables . . . 27

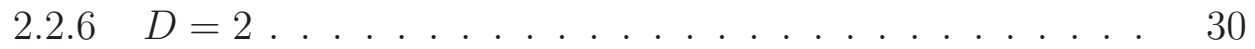

$2.2 .7 \quad D=3 \ldots \ldots \ldots \ldots \ldots \ldots \ldots \ldots \ldots$

2.2 .8 Finite Size Scaling . . . . . . . . . . . . . . . . . . 35

2.2.9 From Discrete To Continuous RG transformation . . . . 45

2.2 .10 Linear Analysis . . . . . . . . . . . . . . . . . . 46

2.2 .11 Window Of Stability . . . . . . . . . . . . . . 51

2.2 .12 Two Lattice Matching . . . . . . . . . . . . . . . 53

3 EXACT RENORMALIZATION GROUP . . . . . . . . . . . 72

3.1 Introduction . . . . . . . . . . . . . . . . . . 72

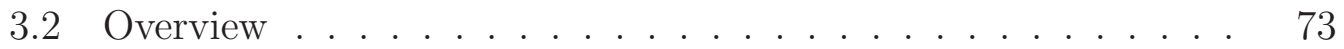

3.3 Numerical Methods To Find The Fixed Points . . . . . . . . 76

3.3.1 Polynomial Expansion of the Potential $u(\rho)$ About the Origin . . . . . . . . . . . . . . . 77

3.3.2 Polynomial Expansion of the Potential $u(\rho)$ About the Minimum . . . . . . . . . . . . . . . 79

3.4 Equivalence between Litim and Wilson-Polchinski equation . . 83

3.5 Effects Of The Cutoff Functions . . . . . . . . . . . 85

4 RENORMALIZATION GROUP AND LATTICE GAUGE THEORIES 92 
4.1 Motivations . . . . . . . . . . . . . . . . 92

4.2 Introduction To Lattice Gauge Theory . . . . . . . . . . . . . . 93

4.3 Finite Size Scaling of The $S U(2)$ Lattice Gauge Theory . . . . 94

4.3.1 Binder cumulant and FSS . . . . . . . . . . . . 95

4.3.2 Determination of the critical exponent $\nu \ldots . . . . .997$

4.3.3 Determination of the critical exponent $\omega . . . . . .103$

4.4 Infrared Fixed Point of the $S U(3)$ Multiflavor Lattice Gauge

Theory ................... . . 104

5 TENSOR RENORMALIZATION GROUP . . . . . . . . . . . 113

5.1 TRG on 2D $O(2)$ model . . . . . . . . . . . . . . . . . . 113

5.2 TRG on 2D $\mathrm{O}(3)$ model . . . . . . . . . . . . . 116

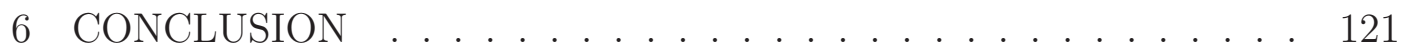

\section{APPENDIX}

A CRITICAL VALUES FOR THE HIERARCHICAL MODEL . . . . 123

B RESCALING OF THE ERG EQUATION . . . . . . . . . . . . . 138

B.1 No A Dependence . . . . . . . . . . . . . . . . . . . . . . 138

B.2 No B Dependence . . . . . . . . . . . . . . . . . . . . . . . 139

B.3 More about $A$ and $B$ dependence . . . . . . . . . . . . . . . . . 140

C LATTICE GAUGE THEORIES . . . . . . . . . . . . . . . . . . 141

D ERROR ANALYSIS OF THE BINDER CUMULANT . . . . . . . . 150

D.1 What We Have . . . . . . . . . . . . . . . . . . . . . 150

D.2 What We Want . . . . . . . . . . . . . . . . . . 150

D.3 What We Have Done . . . . . . . . . . . . . . . . . . 150

D.4 The Proper Way Of Getting The Error . . . . . . . . . . . . . 151

REFERENCES . . . . . . . . . . . . . . . . . . . . 152 


\section{LIST OF TABLES}

3.1 The first six couplings at the fixed point of Eq. (3.14) for the expansion of Eq. (3.21) with $m=45$ and $\rho_{\min }=1.814898403687$. . . .

3.2 The first six critical exponents calculated from the expansion of Eq. (3.21) with $m=42$ and $\rho_{\min }=1.814898403687 . \ldots . . . .$.

A.1 $\gamma$ and $\Delta$ for different $l^{D} \ldots \ldots \ldots \ldots \ldots \ldots \ldots$

A.2 Critical values for different $l^{D} \ldots \ldots \ldots \ldots \ldots \ldots \ldots$

A.3 $\beta_{c}$ for $l^{D}=3$. $l_{\max }$ is the initial and final truncation value; aim is the precision of $\beta_{c} ; t_{\text {val }}$ is the intermediate truncation value. $\ldots \ldots .125$

A.4 $\beta_{c}$ for $l^{D}=3$. $l_{\max }$ is the initial and final truncation value; aim is the precision of $\beta_{c} ; t_{\text {val }}$ is the intermediate truncation value. $\ldots \ldots 126$

A.5 $\gamma$ for $l^{D}=3$. $l_{\max }$ is the initial and final truncation value; aim is the precision of $\beta_{c} ; t_{v a l}$ is the intermediate truncation value. $\ldots \ldots .127$

A.6 $\gamma$ for $l^{D}=3$. $l_{\max }$ is the initial and final truncation value; aim is the precision of $\beta_{c} ; t_{v a l}$ is the intermediate truncation value. $\ldots \ldots 128$

A.7 $\lambda_{1}$ for $l^{D}=3$. $l_{\max }$ is the initial and final truncation value; aim is the precision of $\beta_{c} ; t_{v a l}$ is the intermediate truncation value. . . .

A.8 $\lambda_{1}$ for $l^{D}=3$. $l_{\max }$ is the initial and final truncation value; aim is the precision of $\beta_{c} ; t_{v a l}$ is the intermediate truncation value. . . . .

A.9 $\lambda_{2}$ for $l^{D}=3$. $l_{\max }$ is the initial and final truncation value; aim is the precision of $\beta_{c} ; t_{v a l}$ is the intermediate truncation value. . . .

A.10 $\lambda_{2}$ for $l^{D}=3$. $l_{\max }$ is the initial and final truncation value; aim is the precision of $\beta_{c} ; t_{v a l}$ is the intermediate truncation value. . . . 132

A.11 $\beta_{c}$ for $l^{D}=4$. $l_{\max }$ is the initial and final truncation value; aim is the precision of $\beta_{c} ; t_{v a l}$ is the intermediate truncation value. $\ldots \ldots .133$

A.12 $\gamma$ for $l^{D}=4$. $l_{\max }$ is the initial and final truncation value; aim is the precision of $\beta_{c} ; t_{v a l}$ is the intermediate truncation value. . . . . . .

A.13 $\lambda_{1}$ for $l^{D}=4$. $l_{\max }$ is the initial and final truncation value; aim is the precision of $\beta_{c} ; t_{v a l}$ is the intermediate truncation value. . . .

A.14 $\lambda_{2}$ for $l^{D}=4$. $l_{\max }$ is the initial and final truncation value; aim is the precision of $\beta_{c}$; $t_{v a l}$ is the intermediate truncation value. . . . . 
A.15 $\beta_{c}$ for $l^{D}=5$. $l_{\max }$ is the initial and final truncation value; aim is the precision of $\beta_{c} ; t_{v a l}$ is the intermediate truncation value. . . . . . . 134

A.16 $\gamma$ for $l^{D}=5$. $l_{\text {max }}$ is the initial and final truncation value; aim is the precision of $\beta_{c} ; t_{v a l}$ is the intermediate truncation value. . . . . . 135

A.17 $\lambda_{1}$ for $l^{D}=5$. $l_{\max }$ is the initial and final truncation value; aim is the precision of $\beta_{c} ; t_{v a l}$ is the intermediate truncation value. . . . . 136

A.18 $\lambda_{2}$ for $l^{D}=5$. $l_{\max }$ is the initial and final truncation value; aim is the precision of $\beta_{c} ; t_{v a l}$ is the intermediate truncation value. . . . . 137 


\section{LIST OF FIGURES}

1.1 Real and imaginary parts of Fisher zeros for $D=3$ and $n_{\max }$ going from 2 to 11 and discrete $\beta$ functions for $D=3,2$, and 1.7 . . . .

1.2 RG flows for the $D=3$ hierarchical model in the complex $\beta$ plane obtained by the two lattice method. and close up view of $D=2 \mathrm{RG}$ flows and Fisher zeros for different volumes.

1.3 Density of state related function $h(y)$ together with the expansion in Legendre polynomials up to order 20 and Fisher zeros for the pure gauge SU(2) without an ad-joint term for different volumes [1]. . .

2.1 The partition zeros in the complex $\beta$ plane for the HM with volume $2^{4}$ in 3 dimension.

2.2 The partition zeros in the complex $\beta$ plane for the HM with volume $2^{5}$ in 3 dimension.

2.3 Same system as in Fig. 2.1 but in the complex inverse $\beta$ plane. .

2.4 Same system as in Fig. 2.1 but in the complex inverse $\beta$ plane. .

2.5 The roots of the hierarchical model partition for $D=2$ and $V=2^{4}$ in the $x=e^{\beta}$ plane.

2.6 The roots of the HM partition for $D=2$ and $V=2^{4}$ in the $\log (x)=$ $\beta / 128$ plane.

2.7 The Fisher zeros for the two dimensional HM with different volume $V=2^{N}$; from up to down: $N=2,3,4,5,6,7,8 \ldots \ldots$. . . . .

2.8 The real and imaginary part of the Fisher zeros for the two dimensional HM with different volume $V=2^{N} \ldots \ldots \ldots$

2.9 The Fisher zeros and the critical $\beta$ for the 3 dimensional HM with different volume $V=2^{N}$; from up to down: $\mathrm{N}=2,3,4,5,6,7,8,9$, $10,11$.

2.10 The real and imaginary part of the Fisher zeros for the 3 dimensional HM with different volume $V=2^{N}$

$2.11-\ln L$ versus $\ln \left|\beta(L)-\beta_{c}\right|$ for $n=2-11$; the fitting was done with $n=8-11$.

$2.12-\ln L$ versus $\ln |\operatorname{Im} \beta(L)|$ for $n=2-11$; the fitting was done with $n=8-11$. 
$2.13-\ln L$ versus $\ln \left|\operatorname{Re} \beta(L)-\beta_{c}\right|$ for $n=3-11$; the fitting was done

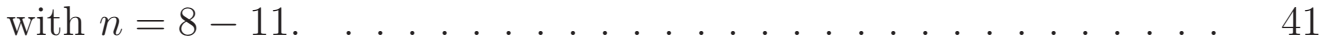

2.14 The density of the zeros is proportional to $\ln V . \quad$. . . . . . . . . . 42

2.15 The real and imaginary parts of the Fisher zeros for $n=2-11$. . . . 44

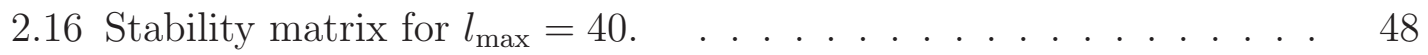

2.17 The fixed point polynomial $R_{0}^{*}$ for different $b^{D}$. . . . . . . 51

2.18 Red dots: $\langle L| R>$; Blue dots: $\langle L| R>$. . . . . . . . . . . . . . 53

2.19 The critical temperature $\beta_{c}$ changes with $b^{D}$. . . . . . . . 54

2.20 The critical exponent $\gamma$ changes with $b^{D}$. . . . . . . . . 55

2.21 An example of blocking for the one dimensional hierarchical model. $\quad 58$

2.22 RG flows obtained by the two lattice matching methods for hierarchical Model. . . . . . . . . . . . . . . . . . . . 61

2.23 Unambiguous $\mathrm{RG}$ flows for the hierarchical model in the complex $\beta$ plane obtained by the two lattice method. . . . . . . . . . . 62

2.24 Three mechanisms for the loss of the fixed point(s) described in [2]. 64

2.25 Discrete $\beta$ function for the hierarchical model with $D=2$ and $f(m)=$ 1. . . . . . . . . . . . . . . . .

2.26 Discrete $\beta$ function for the hierarchical model with $D=3$ and $f(m)=$ 1. ......................... 66

2.27 Discrete $\beta$ function for the hierarchical model with $D=1.9,1.994$, and 2 and $f(m)=\log (m)$. . . . . . . . . . . . . . 67

2.28 Complex RG flows for $D=2 \ldots \ldots \ldots$. . . . . . . . 68

2.29 Complex RG flows for $D=1.994$. . . . . . . . . . . . . . 69

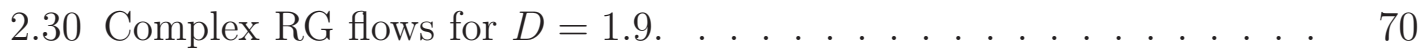

2.31 The density of the zeros is proportional to the $\ln V . \quad \ldots . . . . \quad 71$

3.1 The red dots data were obtained from Fig. 4 in Ref. [3]. . . . . . . 73

3.2 Two $\lambda_{1}$ s are stable with increasing polynomial order $m$. . . . . . . 79 
$3.3 \lambda_{1}$ approaches the asymptotic value $\lambda_{1}(\infty)=-0.18606424947 \cdots$ slowly with increasing polynomial order $m$ by using the expansion around the origin method. . . . . . . . . . . . . . . 80

$3.4 u(\rho)$ versus $\rho . \rho_{\min }$ corresponds to the minimum of the potential $u(\rho) . \quad 81$

$3.5 \lambda_{2}^{\prime}$ approaches the asymptotic value very fast with in creasing polynomial order $m$ by using the expansion around the potential minimum method. . . . . . . . . . . . . . . . . 82

3.6 The convergence of the critical exponent $\nu$. . . . . . . . . . . . . 82

3.7 Critical exponents $\nu$ and $\omega$ change as function of $\epsilon$. . . . . . . . 87

3.8 Critical exponents $\nu$ and $\omega$ for different $\epsilon$. . . . . . . . . . 88

3.9 Show that Litim used formula corresponding to $y^{5 / 2}$ instead of $y^{3 / 2}$ to get the data "DataNuOmega.m". . . . . . . . . . . . . 88

$3.10 \mathrm{y}$ axis is the $\omega$ and $\mathrm{x}$ axis is the $1 / \nu \ldots \ldots$. . . . . . . 89

3.11 Blue points calculated with different $\epsilon_{1}$ and $\epsilon_{2}$; red points are calculated from the hierarchical model with different $b^{D}$. . . . . . . . 90

3.12 Blue points calculated with different $\epsilon_{1}$ and $\epsilon_{2}$; red points are calculated from the hierarchical model with different $b^{D} \ldots \ldots$. . . . . . 91

$4.1 g_{4}$ versus $\beta$ for different volumes. . . . . . . . . . . . . 96

4.2 Linear fit of $g_{4}$ near $\beta_{c}$ for $N_{\sigma}=10,\left(\beta_{c}\right)_{a p p}=2.299$ and $(1 / \nu)_{a p p}=$

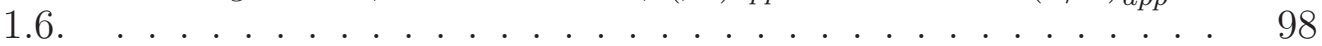

4.3 Determination of $1 / \nu$ from the log-log fit discussed in the text. . . 99

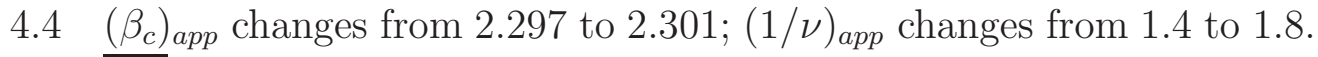
$\overline{1 / \nu}=1.570 ; \sigma=0.027 \ldots \ldots \ldots 10 . \ldots \ldots 1$

$4.5\left(\beta_{c}\right)_{\text {app }}$ changes from 2.298 to $2.300 ;(1 / \nu)_{\text {app }}$ changes from 1.4 to 1.8 . $\overline{1 / \nu}=1.571 ; \sigma=0.028$.

4.6 For $\beta_{c}=2.2991$, the behavior is approximately linear: $g_{4} \simeq g_{4}\left(\beta_{c}, \infty\right)+$

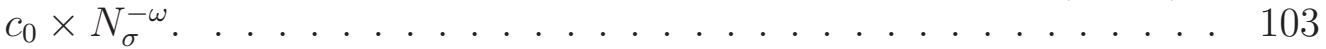

$4.7 \beta_{0}>0$ and $\beta_{1}>0 \ldots \ldots \ldots \ldots$

$4.8 \beta_{0}<0$ and $\beta_{1}<0 \ldots \ldots \ldots \ldots$

$4.9 \beta_{0}>0$ and $\beta_{1}<0 \ldots \ldots \ldots \ldots 6$ 
4.10 Average plaquette for $N_{f}=4$ and $N_{f}=12$ at different volumes. $\quad$. 108

$4.11<\bar{\Psi} \Psi>$ for $N_{f}=4$ and $N_{f}=12$ at different volumes. . . . . . . . 109

4.12 Fisher zeros for $N_{f}=4$ and $N_{f}=12$ at different volumes. . . . . . 110

4.13 Typical plaquette history for the $\mathrm{V}=4^{4}$ system. . . . . . . . . . . 111

4.14 Typical plaquette history for the $\mathrm{V}=6^{4}$ system. . . . . . . . . . . 112

5.1 Tensor representation of the two dimensional $O(2)$ model. . . . . . 114

5.2 Tensor representation of the two dimensional $O(3)$ model. . . . . . 120

C.1 Test for the rational approximation. . . . . . . . . . . . . . . 147

C.2 Test for the rational approximation. . . . . . . . . . . . . . . 147

C.3 Test for the rational approximation. . . . . . . . . . . . . . . . 148

C.4 Test for the rational approximation. . . . . . . . . . . . . . . . 148

C.5 Test for the rational approximation. . . . . . . . . . . . . . 149 


\section{CHAPTER 1 INTRODUCTION}

\subsection{Introduction}

The renormalization group ( $R G$ ) has played an important role in the development of quantum field theories in a variety of settings. It provides an effective way of analyzing which short-distance feature effects long-distance physics. On the other hand, renormalization group methods have also been developed for statistical mechanical systems in order to deal with systems with many degrees of freedom and of strong correlations. It provides a universal language that can be applied over a broad range of areas, i.e., particle physics, condensed matter physics, atomic physics, etc.

The basic idea of RG was introduced by Stueckelberg and Petermann [4] in 1951 in the form of the group transformation of the $S$-matrix $S$

$$
\mathbf{P}_{\mathbf{M} \alpha} S=\frac{\partial S}{\partial \log \epsilon_{M}},
$$

where $\epsilon_{M}$ is the coupling constant and $\mathbf{P}_{\mathbf{M} \alpha}$ is the infinitesimal operator. Two years later, in 1953, the same authors [5] (see also [6]) formulated their ideas more explicitly and introduced the function $h(x, \mu, e)$ in quantum electrodynamics (QED), which can be considered as a first version of the $\beta$ function

$$
\mathbf{P}_{\mathbf{i}} S=h_{i_{e}}(x, \mu, e) \frac{\partial S(x, \mu, e, \cdots)}{\partial e} .
$$

They were able to relate different reparametrizations of QED mass $(x, \mu)$ and electric charge $(e)$ via some transformations. They called them "group of normalization", which is given by infinitesimal operations $\mathbf{P}_{\mathbf{i}}$. Gell-Mann and Low [7] in 1954 obtained the functional equations for QED propagators in the low wavelength/highenergy, or in another word the ultraviolet (UV) limit. They related the physical charge $e$ with the bare charge $e_{0}$ via the family of parameters $e_{\lambda}$ in different $(\lambda \rightarrow 0$, 
$\lambda \rightarrow \infty$ ) limits. Bogoliubov and Shirkov [8] soon after in 1955 - 1956 gave a clearer picture on the connection between the above two groups work and they developed a simple way to analyze the UV and infrared (IR) asymptotic (see also [9]). Not many RG conceptional ideas on the filed theory were developed until the beginning of the 1970s, Politzer [10] and Gross and Wilczek [11] showed that the non-Abelian gauge theories are asymptotically free by showing that the Callan [12] Symanzik $[13,14] \beta$ function has an opposite sign comparing to the QED case. This explained why quarks acted like free particles at small distances. The discovery of the asymptotic freedom strengthened the foundations of the quantum field theory (QFT) and solved the "inconsistency" of QED, as argued by Landau [15]. Those were the early development of the RG in particle physics. More references can be found in $[16,17,18]$.

Wilson $[19,16,20]$ generalized the idea of RG to the theory of the critical phenomena. It provided a general framework to deal with systems with many coupled degrees of freedom, including many interesting systems in the condensed matter physics, statistical physics, and many other different areas. Numerical methods coupled with RG makes the analysis of the properties of the systems very near to the critical points possible.

The Monte Carlo renormalization group (MCRG) method is one of the powerful numerical tools to study critical properties of the spin and gauge systems $[21,22,23,24,25,26,27,28,29,30,31,32,33,34,35,36,37,38,39,40,41,42$, 43, 44, 45]. Applying Monte Carlo methods to the RG study was first suggested by Shang-Keng Ma [46] in the 1970s. The method was extensively used in the 1980s. The two lattice matching method has been proved to be a very useful technique to calculate the non-perturbative $\beta$ function of gauge and spin models. Recently, Hasenfratz [40] has applied the two lattice matching method to many flavor SU(3) 
gauge theories and analyzed critical properties of the theory. This motivated us to study more carefully the MCRG methods on both spin and lattice gauge models. Along this line, we carefully analyzed critical properties of Dyson's hierarchical model, discussed the connection between the continuous and discrete RG method, went through the functional RG, the tensor RG and finally the multi-flavor lattice gauge models.

The plan of the thesis is first to apply the two lattice matching method to the hierarchical model, where simple integral relation is exact. We will try to match a series of couplings and draw flow lines in the coupling space. Moreover, we will extend the flow to the complex coupling space and try to support the idea that Fisher Zeros, the partition function zeros in the complex temperature plane, (see section 2.2.4) form a boundary of the flow. We will eventually apply the technique developed to the lattice gauge models and lattice QCD like models. The structure of the thesis is the following: in the remaining of this chapter, I will briefly introduce some basic ideas used in the following chapters, and give a brief summary on what we have done so far. In Chapter 2, I will start with the conventional Ising model and then move on to Dyson's hierarchical Ising model. We will spend a certain among of time on the hierarchical model. Most of the concepts and techniques used later on will be introduced here. Section 2.2 gives a brief introduction of the hierarchical model, shows the calculation of the critical properties, and discuss the linear analysis; section 2.2.4 summarizes the Fisher zeros calculations done in the hierarchical model; Finite size scaling techniques are discussed in section 2.2.8 and applied to the gauge model. In Chapter 3, I will show some exact renormalization group (ERG) results. The motivation for this chapter is to analyze the discrete and continuous RG transformations. In Chapter 4, I will switch our attention to the more complicated and more interesting lattice gauge models. I will first analyze 
the connection between the Ising model and the $S U(2)$ lattice gauge model by calculating the critical exponents. I will then work on the $S U(3)$ lattice gauge model with many light flavors. Due to the existence of the matter field, the computation is more tricky and time consuming. I will not show the most up-to-date result but only some results shown in 2012 lattice conference. New results will be published soon later. Tensor renormalization group (TRG) method will be discussed in Chapter 5. The advantages of the TRG is that it can be done for arbitrary large volume as long as one can perform the RG transformation once and the accuracy can be systematically improved.

I have been focusing my work on the field of theoretical high energy physics, with particular emphasis on phase transitions of gauge and spin models, and physics beyond the standard model. One of the purpose of my research was to figure out the phase structure of various gauge models and to unambiguously determine the existence of nontrivial infrared fixed point (IRFP) for those models. In order to do so, we have developed new techniques to prove the existence/nonexistence of the IRFP by monitoring the complex zeros of the partition function, which is conventionally called Fisher zeros, as the volume increases. The method was first constructed and tested on spin models and then extended to lattice gauge models, such as pure gauge $S U(2)$ and $U(1)$ case (I was not directly involved in the $U(1)$ case though). During this process, I have been trained to solve problems both analytically and numerically. On the analytical side, RG and especially finite size scaling (FSS) technique has been used through out my whole research period. I have been able to estimate the leading and sub-leading critical exponents for particular spin and gauge models. Finite volume effect of Fisher zeros and discrete $\beta$ functions has also been carefully analyzed. The most important part is that I managed to construct the RG flows in the complex plane and showed that Fisher zeros separate 
flows going into different fixed points.

In the following, I will briefly present my current and further research. With the upcoming experimental effort at Large Hadron Collider (LHC), there has been a lot of recent activities in the lattice community regarding the existence of the non-trivial IRFP in lattice models. The analysis involves non-perturbative dynamics like in QCD. Therefore lattice calculations are essential. Despite the intrinsic theoretical interest of the realization of conformal symmetry near an IRFP, it seems that phenomenologically, the preferred option is a "walking" coupling constant for extended technicolor interactions $[47,48]$.

Recently, many groups have been doing simulations on different systems in order to locate possible IRFP. Most of the simulations were done on $S U(2)$ and $S U(3)$ with different numbers of fermion flavors $N_{f}$ in the fundamental representation. It seems to be uncontroversial that the $\beta$ function is always negative in the range of couplings measured for $S U(3)$ with $N_{f} \leq 8$. The corresponding continuum theory is asymptotically free, chirally broken and confining. For $N_{f}>8$, the situation is somewhat controversial. The Yale collaboration claimed an IRFP for $S U(3)$, $N_{f}=12$, which contradicts the Wuppertal-Budapest collaboration's observation that the systems with $N_{f}=8$ and 12 are chirally broken and confining. For $S U(3)$ with $N_{f}=2$ flavors of sextet fermions, the Colorado-Tel Aviv collaboration first claimed indications for an IRFP but was then disproved by the same authors. For $S U(2)$ with 2 ad-joint representations, there are also indications for the existence of an IRFP in the strong coupling region, but it is not completely uncontroversial either.

Given this situation, it is necessary to develop new methods that would lead to an unambiguous determination of the existence of IRFP or of a non-perturbative "walking" coupling constant. 
On the theoretical side, developing new methods and applying existing RG ideas, which are usually tested on relative simple spin models, to the complicated gauge models are essential. Functional RG has been very successfully applied to many branches of physics. It is demanding to seek its application to every field of physics, especially the newly emerged ultra-cold atoms (optical lattice).

\subsubsection{Spin Models}

The Hierarchical Ising model (HM) is an ideal laboratory to test various ideas before one applies them to the more complicated full QCD case. Depending on the dimensionality and the interaction strength among blocks, the HM can have second order phase transition, no phase transition, or have a Thouless effect like in the Anderson model [49]. Fisher zeros and critical exponents of the HM can be calculated with very high accuracy. Figure 1.1 (Left Panel) shows the real and imaginary part of the lowest Fisher zeros for $D=3 \mathrm{HM}$. The lowest Fisher zeros accumulate toward the critical value $\beta_{c}$ as the volume increases. The departure from a linear behavior is significant and requires sub leading corrections. The dotted lines are the fittings with sub leading corrections included.

The role of Fisher zeros can be clearly seen from the construction of the RG flows in the complex parameter plane. Complex RG flows can be constructed by using a two-lattice matching method. This method is a way of measuring the running of the bare couplings based on the fact that all the observables will have the same value if the models have the same effective action. Figure 1.2 shows complex flows for different dimensions. It can be clearly seen that Fisher zeros form the basin of attraction of the IRFP $[50,51]$. That means that one only needs to

get Fisher zeros of the system in order to determine whether there is a IRFP or not [52]. Figure 1.2 (Right Panel) also shows that Fisher zeros for larger volumes move approximately "backward" along the separation flow lines. This behavior 

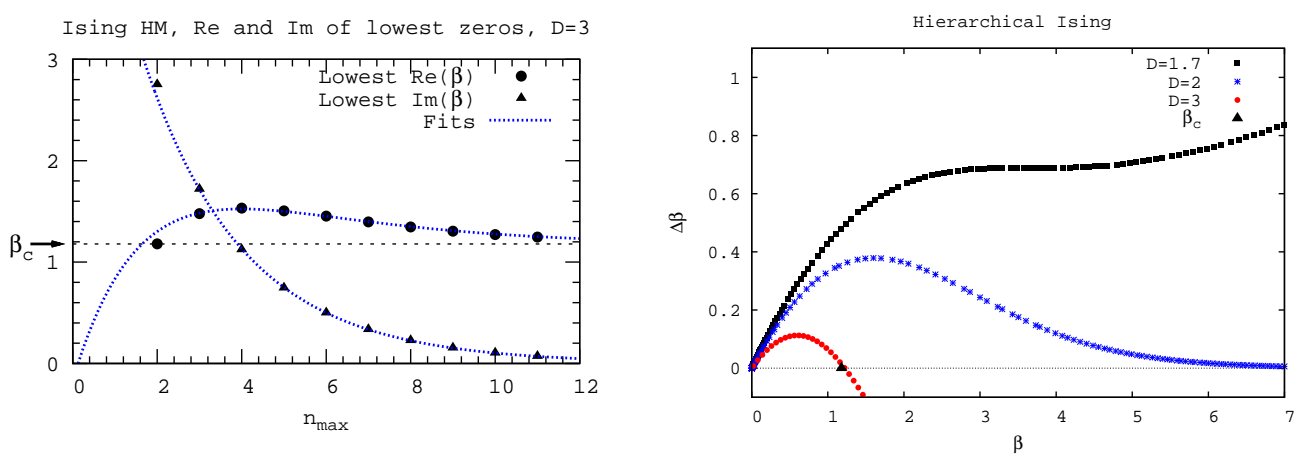

Figure 1.1: Real and imaginary parts of Fisher zeros for $D=3$ and $n_{\max }$ going from 2 to 11 and discrete $\beta$ functions for $D=3,2$, and 1.7 .

approximately agree with the argument that Fisher zeros for $2^{n}$ sites should map into Fisher zeros for $2^{n-1}$ sites.

Calculating the discrete $\beta$ function is one of the methods used to look for or exclude IRFP. Discrete $\beta$ function of the HM can be calculated numerically and semi-analytically. The zero of the discrete $\beta$ function signals the appearance of a fixed point. However, sometimes finite volume effect may generate pseudo fixed point. This has been discussed extensively recently [49]. If one is interested in the corresponding continuous Callan-Symanzik $\beta$ function, for example in the gauge model, one could use functional conjugation method to construct it from the discrete one $[53,49]$.

\subsubsection{Gauge Models}

In order to locate Fisher zeros for gauge models, we start from the inverse Laplace transform of the partition function, which is also called the density of state (DOS). The DOS can be constructed from plaquette configurations generated from the Monte Carlo simulation. The DOS of pure gauge $S U(2)$ on $L^{4}$ lattice has been successfully constructed $[54,55]$, see Fig. 1.3 (Left Panel). Once the DOS is known, 

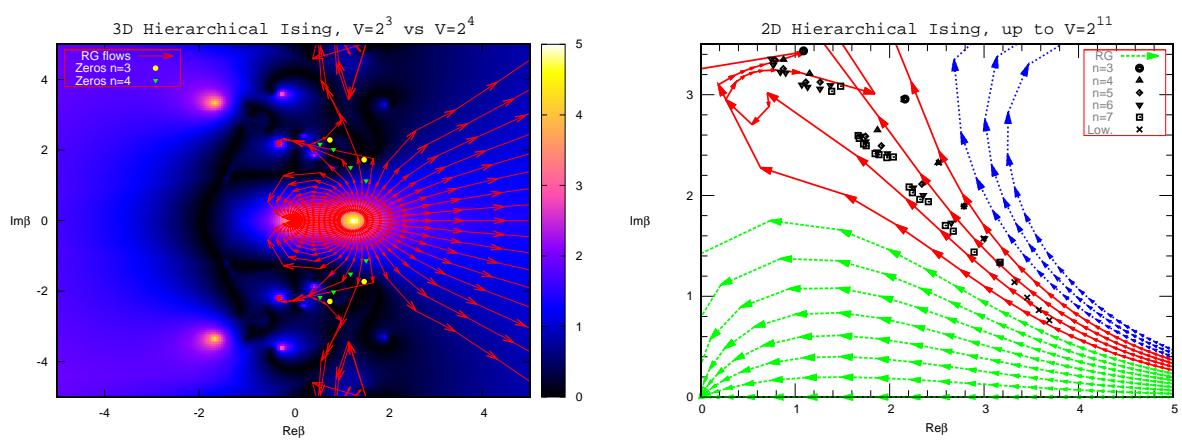

Figure 1.2: RG flows for the $D=3$ hierarchical model in the complex $\beta$ plane obtained by the two lattice method. and close up view of $D=2 \mathrm{RG}$ flows and Fisher zeros for different volumes.
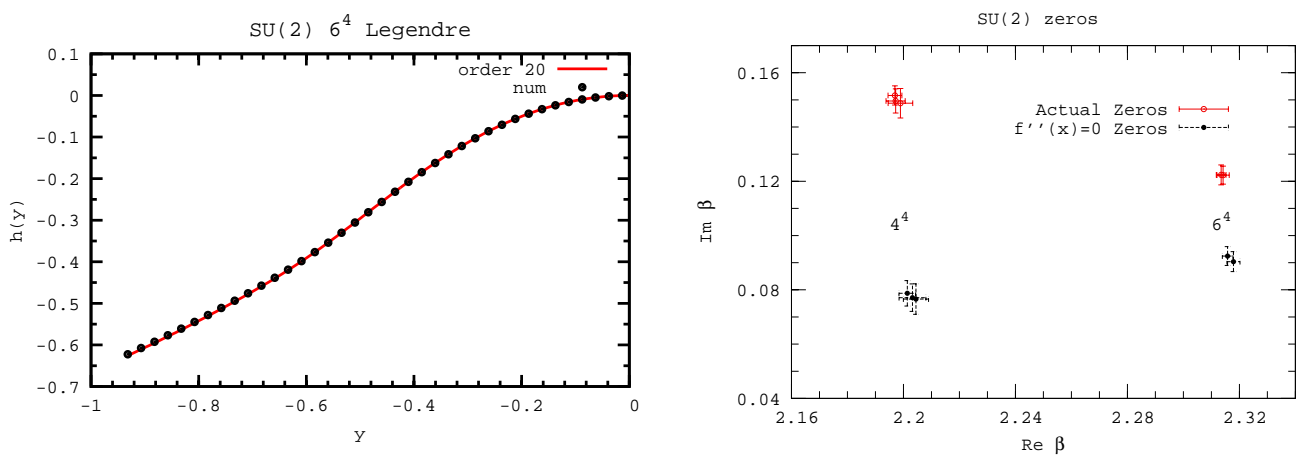

Figure 1.3: Density of state related function $h(y)$ together with the expansion in Legendre polynomials up to order 20 and Fisher zeros for the pure gauge SU(2) without an ad-joint term for different volumes [1].

it is possible to calculate the partition function for any complex value of $\beta$, where $\beta=2 N / g^{2}$. Fisher zeros can then be calculated by using the re-weighting method. Figure 1.3 (Right Panel) shows Fisher zeros for pure $S U(2)$ gauge models on two different volumes. $S U(2)$ with ad-joint term and $U(1)$ cases have also been done [1]. The fact that Fisher zeros stabilizes away from the real axis for $S U(2)$ and pinches the real axis for $U(1)$ signals different cases of phase transitions. Similar phenomena have also been well tested in spin models [52]. 
Since we are ultimately interested in the thermodynamic limit, finite size scaling (FSS) technique is essential in analyzing the data and extrapolating relevant quantities to the infinite volume limit. Considerable experience on FSS technique has been acquired and already been applied to the analysis of the spin and gauge models $[52,56]$. Leading and sub leading critical exponents of the gauge model have been extrapolated from the data by calculating the 4th order Binder cumulant.

\section{$1.2 \quad$ Research Results}

- Gauge Models

I have applied the techniques discussed above to $S U(3)$ lattice gauge model with various fermion flavor numbers and further located the partition function zeros of these models. This will provide an alternative way to locate the conformal window non-pertubatively.

We have a working parallel code with Dr. Don Sinclair to simulate $S U(3)$ with 2 and 3 staggered fermions. I have modified this existing code to simulate with $N_{f}$ going from 4 to 16 and possibly non integer $N_{f}$ by using rational hybrid Monte Carlo (RHMC) algorithm. The $N_{f}=8$ and 16 case will be dealt with HMC algorithm separately. I started from small volume $4^{4}$ with $N_{f}=3$, where the rough location of $\beta_{c}$ is known, and gradually increased the number of flavors to monitor how the $\beta_{c}$ changes with the number of flavors. I have generated data for higher volumes. All the codes developed for this purpose are working well now and have adaptive routines. It would be good if we could compare results with other fermion actions. Therefore, we also use Professor Anna Hasenfratz group's code, which is based on the publicly available MILC code, to investigate the role of $\mathrm{nHYP}$ and APE spearing on the effects of the phase transitions. 
- Spin Models

I have two undergoing projects in hand. One is the "numerical instabilities associated with block spinning non-integer numbers of sites", where we block spin non-integer number of sites at each step and try to push the block spinning number to the continuum limit 1, where Functional RG methods work. We found out that the result is numerically unstable which may due to the logarithmic divergent term. Another one relates to the "exact renormalization group" (ERG) equations. I found out that some ERG equation are invariant under some particular rescaling.

Up to now, we have constructed complex RG flows for models with second order phase transition $(D=3 \mathrm{HM})$ and with no phase transition $(D=2 \mathrm{HM})$. I would like to find a suitable model with first order phase transition and see how the flows behave.

\section{- Tensor Renormalization Group}

The RG is a beautiful theory in dealing with systems with different length scales. It has inspired new concepts and computational methods in many branches of physics. In the lattice gauge theory, however, we recently found out that the conventional Migdal-Kadanoff (MK) method does not serve the purpose of high accuracy calculations due to its crude "bond sliding" approximation [57]. This inspired us to to develop new method to "block spin" the system accurately and provide a tool to study lattice models near conformality. We started out noticing the density matrix renormalization group (DMRG) [58] methods which are widely used in the condensed matter community. We realized that despite its success in dealing with quantum systems in one space and one time dimension, the method quickly fails in dealing with higher dimensions. Recently, we developed the tensor renormalization group 
(TRG) $[59,60]$ to deal with classical discrete spin models away from criticality. This inspired us to develop new methods to deal with continuous spin and lattice gauge models based on TRG. A variant of that, second renormalization of tensor-network state (SRG), which takes into account the effect of the environment and improves the accuracy at the criticality is also under development. Our goal is to apply the method to four dimensional lattice gauge models with fermions.

This is a long term project partially due to its complexity in four space-time dimension. Up to now, we have successfully written down the tensor state and figured out how to perform contractions for the two and three dimensional $O(N)$ models, and three dimensional $U(1)$ model. New mathematical and computational tools may be needed to deal with such complicated cases. 


\section{CHAPTER 2 \\ RENORMALIZATION GROUP METHODS ON SPIN MODELS IN THE HIERARCHICAL APPROXIMATION}

\section{$2.1 \quad$ Ising Model}

Before discussing about the hierarchical model, let us start with the usual Ising model. The Ising model was first proposed by Lenz [61] to his student Ising [62] in the early 1920s in order to analyze ferromagnetism. The hope was to devise a model with inter-particle interaction to display a magnetic phase transition. Ising managed to solve the model on a one-dimensional lattice and found that the model actually had no phase transition at finite temperature. We now sometimes say that the one dimensional Ising chain has a phase transition at zero temperature since the system only has long range order at $T_{c}=0$. The 'failure' of describing the phase transition made Ising conclude that the model would not have any phase transition in higher dimension either. Of course, we now know that the Ising model has phase transitions in two and higher dimensions. In 1936, Peierls [63] proved that the Ising model should have phase transitions in two and three dimension at sufficiently low temperatures. Later in 1941, Kramers and Wannier [64] successfully located the exact critical temperature of the two dimensional Ising model. They argued that the nearest neighbor Ising model partition function at temperature $T_{1}$ could be transformed into another Ising model at a different temperature $T_{2}$. They identified the critical temperature $T_{c}$ by assuming that there is only one critical temperature. In 1944, Onsager[65], derived the explicit expression of the free energy without external magnetic field in two dimension and further got the singularity behavior of the specific heat, which put Van der Waals mean field theories $[66,67,68]$ into question and led to the discovery of the $\lambda$ transition of the liquid helium [69] and the liquid vapor critical point [70] in Argon. After Onsager's tremendous success, 
many other two dimensional lattice models have been solved exactly. However, none of those different approaches can be generalized to the more physically interesting three dimensional case. In 1952, Lee and Yang [71, 72] gave a rigorous mathematical description of the phenomena of the phase transition. They studied the lattice gas model, which can be mapped into Ising model, in an external magnetic field. They discovered that the complex zeros of the partition function lie on a unit circle in the complex activity plane, or in another word on the imaginary external magnetic field $h$ plane. They could relate the phase transition to the pinching of the zeros onto the real $h$ axis. It was natural to generalize Lee-Yang's theorem to the complex temperature plane. There is no known general theorem to constrain the distribution of the partition function zeros in the complex temperature plane. However, Fisher [73] first observed that the zeros of the two dimensional Ising model on the square lattice distribute on two circles in the complex $\tanh (\beta)$ plane. We usually call the the zeros in the $h$ plane Lee-Yang zeros and the ones in the $\beta$ plane Fisher zeros. In the following, we will briefly discuss the Ising model and use that to introduce important concepts and quantities discussed later in the thesis.

The Hamiltonian of the nearest neighbor Ising model on a regular lattice with external magnetic field $h$ is defined as

$$
H=-J \sum_{<i, j>} \sigma_{i} \sigma_{j}-h \sum_{i} \sigma_{i}
$$

where $\sigma_{i}= \pm 1$, and the first sum is over all the nearest neighbor pair $\langle i, j\rangle$. $J$ is the coupling constant, which controls the interaction strength of the neighboring sites. We usually consider $J>0$ case and the interaction is ferromagnetic. The 
partition function can then be written as

$$
\begin{aligned}
Z[\beta, h] & =\sum_{\left\{\sigma_{i}\right\}} e^{-\beta H} \\
& =\sum_{E=0}^{N_{1}} \sum_{M=0}^{N_{2}} \alpha(E, M) x^{E} y^{M},
\end{aligned}
$$

where $\beta$ is the inverse temperature $T, x=e^{-2 \beta J}$ and $y=e^{-2 \beta h} . N_{1}$ and $N_{2}$ are total number of sites and bonds of the lattice, and $\alpha(E, M)$ is the number of the configuration with $E$ sites and $M$ bonds [74].

The lattice gas Hamiltonian is the following

$$
H=-4 J \sum_{<i, j>} \rho_{i} \rho_{j}-\mu \sum_{i} \rho_{i},
$$

where $\rho_{i}=0,1$, and $\mu$ is the chemical potential. The lattice gas model can be transformed into the Ising model by the following variable changes [71] :

$$
\begin{aligned}
\rho_{i} & =\frac{1}{2}\left(\sigma_{i}+1\right), \\
\mu & =2 h-4 q J,
\end{aligned}
$$

where $q$ is the total lattice site number.

In the zero magnetic field case, $h=0$, the Ising model is in the ferromagnetic phase for $T<T_{c}$, where there is a spontaneous symmetry breaking, and in the paramagnetic phase for $T>T_{c} . T_{c}$ is the so-called critical temperature of the system. For the lattice gas model, there are also gas and liquid phases for different temperatures for $\mu=-4 q J$. I will not go through the exact solution of the two dimensional Ising model here. They can be found in original papers $[65,75]$ and text books $[76,77,78,79]$. Despite the tremendous success of Onsager's exact solution [65], the Ising model and its variants were still considered as the pure mathematical trick which had no physical meaning. Until the 1960s, people realized that the lattice gas model can reasonably well describe the critical behavior of the real liquid gas transition. Combining with the "universality class" and finite size scaling (FSS) 
concepts, Ising model and other 'simple' modes have since been considered seriously as approximation of the real world.

\subsection{Dyson's Hierarchical Model}

In the previous section, we introduced the Ising model and explained that the model can only have phase transitions in the two or higher dimensions. From the universality point of view, the properties of the transition is only related to the dimensionality of the system and the internal symmetries. but not to the detailed structure of the model. Keep in mind that the model we introduced has only nearest neighbor interactions. It has been proved by Peierls [63] that for the ferromagnetic system with short range interactions between the spins, the order-disorder transition requires at least two dimensions. Colin Thompson and Mark Kac conjectured in 1967-1968 that there could be a phase transition for the one dimensional system with interaction strength proportional to $d^{-\alpha}$ with $d$ to be the separation between two spins and $1<\alpha \leq 2$. The conjecture has been proved by Frohlich and Spencer [80] in 1987. But at that time it was still a mystery [81]. Dyson happened to know the conjecture and wanted to prove it. To achieve that, he constructed a special model with long range interaction. He was able to prove that with the interaction strength $d^{-\alpha}$, the system could have a phase transition for $1<\alpha<2$ but he could not prove the $\alpha=2$ case $[82,83,84]$. The $\alpha=2$ case is in fact very interesting because it corresponds to the Anderson model with inverse square interaction $[85,86,87]$. Thouless [88] predicted that the Anderson model should have a very unusual phase transition with discontinuous spontaneous magnetization, which is usually called "Thouless effect". I will discuss about that later in this chapter.

The tool Dyson used was called the hierarchical model (HM). It was first in-

troduced by Freeman J. Dyson [89] in order to mimic a one dimensional Ising model with long-range interactions. It has been proved that the model has a second order 
phase transition under certain conditions. Related models were re-investigated by George A. Baker [90]. His motivation was to find a model where Kenneth G. Wilson's [91] approximate recursion formula was exact. He also extended the model to higher dimensions. Because of its complexity (it has phase transition) and simplicity (it has an exact recursion formula), the model has been investigated intensively by P. M. Bleher and Ja. G. Sinai [92, 93], G. Gallavotti etc [94, 95], P. Collet and J. -P. Eckmann [96, 97, 98], and some others [99, 100, 101, 102, 103, 104, 105, 106, $107,108,109,110,111,112,113,114,115,116,117]$ in the past forty years. For a recent review, see Ref. [118] and the references therein.

\subsubsection{Definition Of The Model}

The partition function of the HM is defined as:

$$
Z=\int e^{-\beta H}
$$

where $H$ is the Hamiltonian of the model $[82,90]$

$$
H=-\frac{1}{2} \sum_{m=1}^{N}\left(\frac{c}{4}\right)^{m} \sum_{p_{N}, \ldots, p_{m+1}}\left(\sum_{p_{m}, \ldots, p_{1}} \phi_{\left(p_{N}, \ldots p_{1}\right)}\right)^{2}
$$

The meaning of the parameters and the notations used in the following sections are defined as the following:

$D$ : Dimension of the system.

$\phi:$ Scalar field in the configuration space.

$\frac{c}{4}=b^{-2-D}:$ Control the decay of the interaction.

$b^{D}$ : Number of sites blocked in each RG iteration.

$a$ : Lattice Spacing.

$\Lambda:$ UV cutoff.

where $\phi$ is the scalar field in the configuration space. For the Ising model 
discussed below, $\phi$ can only be \pm 1 . The index $p_{i}$ is set to 0 or 1 , depending on which block we are referring to, and the summation is over all the combination of the 0 and 1 [118]. The parameter $c$ controls the strength of the interaction. In order to have a phase transition, we require the parameter $\mathrm{c}$ to be positive and smaller than 2. That means that the interaction strength decreases with the distance. The model was initially developed as a one dimensional chain model. It is very easy to extended the original model to higher dimensions. We can include the dimension $D$ to the strength parameter $c: c / 4=b^{-2-D}$, where $b$ is the rescaling factor. For reasons why we can do so, please refer to [118]. Usually we will take $b^{D}=2$ without further notice. One of the properties of the model is that the partition function $Z=\sum e^{-\beta H}$ can be calculated iteratively. In order to get the action, we need to sum up all the spin configuration of the system, or equivalently integrate over a local measure. For the Ising case, the spins can only have the value \pm 1 . Therefore, the local measure for the Ising case is

$$
W_{0}(\phi)=\delta\left(\phi^{2}-1\right) .
$$

In the following, we always work with the Ising measure. The model can also be constructed with Landau-Ginsburg measure

$$
W_{0}(\phi)=\exp \left(-\frac{1}{2} m^{2} \phi^{2}-\lambda \phi^{4}\right) .
$$

In the rest of this section, we will discuss the local measure in both the configuration $\phi$ space and the Fourier transformed $k$ space. Suppose we start from the initial Ising measure in $\phi$ space

$$
\begin{aligned}
W_{0}(\phi) & =\delta\left(\phi^{2}-1\right) \\
& =\frac{1}{2}(\delta(\phi-1)+\delta(\phi+1)) .
\end{aligned}
$$

When we do the renormalization group (RG) transformation, we keep the sum of the spin in one block constant. We can easily get the new measure from the old one 
due to the hierarchical structure of the model:

$$
\begin{aligned}
W_{n+1}(\phi)= & {\left[\int d \phi_{1} d \phi_{2} \delta\left(\phi_{1}+\phi_{2}-\phi\right) *\right.} \\
& \left.W_{n}\left(\phi_{1}\right) W_{n}\left(\phi_{2}\right)\right] * e^{\frac{\beta}{2}\left(\frac{c}{4}\right)^{n+1} \phi^{2}},
\end{aligned}
$$

where $W_{n}$ is the unnormalized probability distribution of $\phi$ in a block of size $2^{n}$.

In order to get rid of the delta function in equation 2.13, we introduce a new variable

$$
\xi=\frac{\phi_{1}-\phi_{2}}{2}
$$

and write

$$
\begin{aligned}
& \phi_{1}=\frac{\phi+2 \xi}{2} \\
& \phi_{2}=\frac{\phi-2 \xi}{2} .
\end{aligned}
$$

Then we can change variable from $\phi_{1}$ and $\phi_{2}$ to $\phi$ and $\xi$. Equation 2.13 becomes

$$
W_{n+1}(\phi)=\left[\int d \xi W_{n}\left(\frac{\phi}{2}+\xi\right) W_{n}\left(\frac{\phi}{2}-\xi\right)\right] * e^{\frac{\beta}{2}\left(\frac{c}{4}\right)^{n+1} \phi^{2}} .
$$

This is the iteration formula for the local measure in $\phi$ space. If we introduce the Fourier transform of the measure $W_{n}(\phi)$

$$
W_{n}(\phi)=\int \frac{d k}{2 \pi} e^{\mathrm{i} k \phi} R_{n}(k),
$$

we will get

$$
\begin{aligned}
R_{n+1}(k)= & \int d \phi e^{-\mathrm{i} k \phi} W_{n+1}(\phi) \\
= & \int d \phi e^{-\mathrm{i} k \phi} e^{\frac{\beta}{2}\left(\frac{c}{4}\right)^{n+1} \phi^{2}} * \\
& {\left[\int d \xi \int \frac{d k_{1} d k_{2}}{(2 \pi)^{2}} e^{\mathrm{i} k_{1}\left(\frac{\phi}{2}+\xi\right)} R_{n}\left(k_{1}\right)\right.} \\
& \left.e^{\mathrm{i} k_{2}\left(\frac{\phi}{2}-\xi\right)} R_{n}\left(k_{2}\right)\right] .
\end{aligned}
$$


Equation 2.19 can be rewritten as

$$
\begin{gathered}
\\
\int d \phi e^{-\mathrm{i} k \phi} e^{\frac{\beta}{2}\left(\frac{c}{4}\right)^{n+1} \phi^{2}} \ldots \\
=\quad \int d \phi e^{-\frac{\beta}{2}\left(\frac{c}{4}\right)^{n+1} \nabla_{k}^{2}} e^{-\mathrm{i} k \phi} \ldots
\end{gathered}
$$

Integrating out $\xi$ in equation 2.19 , the rest becomes

$$
\begin{aligned}
& \int d \xi \int \frac{d k_{1} d k_{2}}{(2 \pi)^{2}} e^{\mathrm{i} k_{1}\left(\frac{\phi}{2}+\xi\right)} R_{n}\left(k_{1}\right) e^{\mathrm{i} k_{2}\left(\frac{\phi}{2}-\xi\right)} R_{n}\left(k_{2}\right) \\
= & \int \frac{d k_{1} d k_{2}}{(2 \pi)^{2}} 2 \pi \delta\left(k_{1}-k_{2}\right) e^{\mathrm{i}\left(k_{1}+k_{2}\right) \frac{\phi}{2}} R_{n}\left(k_{1}\right) R_{n}\left(k_{2}\right) \\
= & \int \frac{d k_{1}}{2 \pi} R_{n}^{2}\left(k_{1}\right) e^{\mathrm{i} k_{1} \phi} .
\end{aligned}
$$

Combining the above two equations, the recursion formula for the Fourier transform of the measure can be written as

$$
R_{n+1}(k)=e^{-\frac{\beta}{2}\left(\frac{c}{4}\right)^{n+1} \nabla_{k}^{2}} R_{n}^{2}(k)
$$

And the Fourier transform of the initial measure $W_{0}$ (Eq: 2.12) is

$$
R_{0}(k)=\cos (k)
$$

We can get the measure for volume $V=2^{N}$ by starting from $R_{0}(k)$ and applying the recursion formula $2.22 \mathrm{~N}$ times. The nice property of the HM is that the block spin transformation only changes the local measure:

$$
W_{n+1}(\phi)=C_{n+1} e^{\frac{\beta}{2}\left(\frac{c}{4}\right)^{n+1} \phi^{2}} \int d \phi^{\prime} W_{n}\left(\frac{\left(\phi-\phi^{\prime}\right)}{2}\right) W_{n}\left(\frac{\left(\phi+\phi^{\prime}\right)}{2}\right) .
$$

And the recursion formula for the $R_{n}(k)$ is then

$$
R_{n+1}(k)=C_{n+1} \mathrm{e}^{-\frac{1}{2} \beta \frac{\partial^{2}}{\partial k^{2}}}\left(R_{n}\left(\sqrt{\frac{c}{4}} k\right)\right)^{2} .
$$

\subsubsection{Partition Function Of The hierarchical Model}

In the following, we will give an example on how to construct the partition function with volume $V=2^{2}$ from that with volume $V=2^{1}$. The Hamiltonian in 
equation 2.8 with $N=2$ is

$$
\begin{aligned}
H_{N=2}= & -\frac{1}{2}\left[\frac{c}{4} \sum_{p_{2}}\left(\phi\left(p_{2}, 0\right)+\phi\left(p_{2}, 1\right)\right)^{2}\right. \\
& \left.+\left(\frac{c}{4}\right)^{2}(\phi(0,0)+\phi(0,1)+\phi(1,0)+\phi(1,1))^{2}\right] \\
= & -\frac{1}{2}\left[\frac{c}{4}\left((\phi(0,0)+\phi(0,1))^{2}+(\phi(1,0)+\phi(1,1))^{2}\right)\right. \\
& \left.+\left(\frac{c}{4}\right)^{2}(\phi(0,0)+\phi(0,1)+\phi(1,0)+\phi(1,1))^{2}\right],
\end{aligned}
$$

and the corresponding partition function is

$$
\begin{aligned}
Z_{N=2}= & \sum e^{-\beta H_{N=2}} \\
= & \sum \exp \left[\frac{\beta}{2} \frac{c}{4}(\phi(0,0)+\phi(0,1))^{2}\right] * \\
& \exp \left[\frac{\beta}{2} \frac{c}{4}(\phi(1,0)+\phi(1,1))^{2}\right] * \\
& \exp \left[\frac{\beta}{2}\left(\frac{c}{4}\right)^{2}(\phi(0,0)+\phi(0,1)+\phi(1,0)+\phi(1,1))^{2}\right],
\end{aligned}
$$

where the summation is over all configurations with $\phi\left(p_{2}, p_{1}\right)= \pm 1$. The partition function for $N=1$ is

$$
\begin{aligned}
Z_{N=1} & =\sum e^{-\beta H_{N=1}} \\
& =\sum_{\phi\left(0, p_{1}\right)= \pm 1} \exp \left[\frac{\beta}{2} \frac{c}{4}(\phi(0,0)+\phi(0,1))^{2}\right] .
\end{aligned}
$$

We notice that equation 2.33 has the same form as equation 2.30 and 2.31. Suppose we have $Z_{N=1}$ already, we only need to calculate equation 2.32 in order to get $Z_{N=2}$. Furthermore, we can construct $Z_{N=i+1}$ from $Z_{N=i}$. That enables us to calculate the partition function exactly for relatively large volume system without running over all the possible configurations.

\subsubsection{Extension Of The Recursion Formula}

The recursion formula 2.25 can be extended for an arbitrary scale transformation. The number of sites integrated for the HM, namely 2, appears as the exponent in the recursion formula. In order to generalize the formula to arbitrary dimension 
$D$, we only need to do the following replacements

$$
\begin{aligned}
& 2 \rightarrow b^{D} \\
& \frac{c}{4} \rightarrow b^{-2-D} .
\end{aligned}
$$

And under the renormalization group ( $R G$ ) transformation, the lattice spacing $a$ and the the momentum cutoff becomes

$$
\begin{aligned}
a & \rightarrow b a \\
\Lambda & \rightarrow \frac{\Lambda}{b} .
\end{aligned}
$$

The recursion formula 2.25 becomes

$$
R_{n+1}(k)=C_{n+1} \mathrm{e}^{-\frac{1}{2} \beta \frac{\partial^{2}}{\partial k^{2}}}\left(R_{n}\left(b^{-(D+2) / 2} k\right)\right)^{b^{D}} .
$$

The usual recursion formula is obtained for $b=2^{1 / 3}$ and $D=3$.

\subsubsection{Zeros Of The Partition Function}

Before starting to address the technical details of how to locate the zeros of the partition function, we would like to discuss the importance of finding the zeros of the partition function, or more generally the zeros of some analytic function.

The first thing coming out of my mind when talking about the zeros of the analytic function is Riemann hypothesis. It states that all the nontrivial zeros of the Riemann zeta function $\zeta(z)$ lies on the critical line $\operatorname{Re}(z)=1 / 2$. Although trillions of zeros have been calculated and all of them lie in the critical line, the hypothesis is still one of the most important unsolved mathematical problems. Another example relating to the zeros of the analytic function is the famous Lee-Yang theorem $[119,120]$. The theorem states that the partition function zeros of the Ising ferromagnet are purely imaginary in the complex magnetic field plane, or lie on the unit circle in the complex $e^{h}$ plane. They also pointed out the relationship between the distribution of the zeros and the order of the phase transition. The theorem has 
been tested on numerous models by different authors. Years later in 1964, M. E. Fisher studied the zeros of the partition function in the complex temperature plane [121]. As mentioned in the previous section, we called these two types of partition function zeros Lee-Yang zeros and Fisher zeros. In this section, we will discuss the later one.

In general, the partition function of the Dyson's hierarchical model (HM) can not be expressed as a finite order polynomial of the variable $e^{-2 \beta}$ or others as can be done in the regular Ising model. This property makes the analysis of distribution and scaling of the partition function zeros more difficult. Whether there is a regular distribution function $g(\theta)$ or not is still an open question.

The partition function of the hierarchical Ising model has the following form

$$
Z[\beta]=\sum_{k=0}^{l} a_{k} e^{\lambda_{k} \beta} .
$$

The coefficient $a_{k}$ are non-negative integers and $\lambda_{k}$ are non-negative real numbers. Equation (2.39) is not an ordinary polynomial but a quasi-polynomial [122]. The general form of the quasi-polynomial is

$$
Z[\beta]=\sum_{j=0}^{m} \sum_{k=0}^{l} a_{j k} \beta^{j} e^{\lambda_{k} \beta} .
$$

Equation (2.39) is a special case with $m=0$. Obviously, there is no real solution for the equation $Z[\beta]=0$ because all the coefficients $a_{k}$ in equation 2.39 are nonnegative. In order to find the partition function zeros, it is necessary to extend $\beta$ to the complex plane. These zeros are the Fisher zeros mentioned above.

We have discussed how to get the exact expression for the partition function in section 2.2.2. In principle, the problem of finding the Fisher zeros is just the problem of finding the roots of the quasi-polynomial 2.39. If all the $\lambda_{k}$ were integers, we could write the partition function 2.39 as a finite order polynomial with the variable $e^{a \beta}$, where $a$ is an integer depending on the actual form of the partition. Many system 
studied have the above properties, i. e. [74]. Finding the roots of the finite order polynomial is not too difficult as long as the order is not too large. The model we are interested in here, however, is not so simple. Most of the times $\lambda_{k}$ is not integer and not even an rational number for the HM. This makes the calculation of the Fisher zeros not so trivial. Even though we could get the expression of the partition function, it is impractical to calculate its roots analytically. We developed two methods to locate the Fisher zeros. The first method is by calculating both the real and imaginary part of the partition function, finding the zeros of each part, and locating their crossing. The second one is the "logarithmic residue" method which is based on the Cauchy's Residue Theorem. The method relies on the following fact

$$
\frac{1}{2 \pi \mathrm{i}} \oint_{C} \beta^{n} \frac{Z^{\prime}(\beta)}{Z(\beta)}=\sum_{i=1}^{k} \beta_{i}^{n},
$$

where $\beta_{i}$ are all the zeros in the integration region $C$. It should be noted that Riemann used similar techniques to estimate the number of zeros in the particular region in his original paper on Riemann hypothesis. The "logarithmic residue" method works well if we know the expression of the function and if we only need to find one or several roots in a specified region, which is just the case we studied here. We usually start from $n=0$ and the right hand side of the equation 2.41 gives the number of roots located in the specified region $C$

$$
\frac{1}{2 \pi \mathrm{i}} \oint_{C} \frac{Z^{\prime}(\beta)}{Z(\beta)}=k \text {. }
$$

We can always adjust the integration region $C$ and make sure that there is only one zero in the region. And then we can set $n=1$ to calculate the location of the zero.

For the lattice gauge models that will be discussed in the following chapters, one usually can not get the exact expression of the partition function. In this case, one can first construct the density of state and get the zeros of the partition function out of the numerical Laplace transformation. 
Let us look at some examples of the first zero searching method. In order to locate the complex zeros of the partition function, we extend the parameter $\beta$, which corresponds to the inverse temperature of the system, to the complex plane. We scan the the complex $\beta$ plane in some region. For every complex $\beta$, we calculate the real and imaginary part of the partition function $Z$. We record the points where the partition function changes sign. These points correspond to the zeros of the real or imaginary part of the partition function. Only the points with zeros in both real and imaginary parts of the partition function are the complex Fisher zeros. Because of the special structure of the equation 2.8, we do not need to sum up all the spin configuration of the system. We can build the Hamiltonian iteratively and calculate the partition function precisely.

Figure 2.1 shows the zeros for $D=3$ and the volume $V=2^{4}$. The Fisher zeros correspond to the crossing of the red and the blue lines. In the region we plotted, there are four Fisher zeros. They are not scattered everywhere into the complex plane. Instead, there is a clear tendency that they follow in some curve. As we increase the volume to $2^{5}$, the Fisher zeros in the same region double (see Fig. 2.1) and the lowest zero moves closer to the real axis. This is in agreement with the fact that there will be no zero in the real axis for a finite volume system. When we go to the infinite volume limit, the zeros pinch to the real temperature axis, which is the indication of the phase transition.

In order to see all the the zeros in the complex temperature plane, we also search the partition function zeros in the complex $\frac{1}{\beta}$ plane. The result for $V=2^{4}$ is shown in Figure 2.3, 2.4. Clearly, all the Fisher zeros accumulate in the small region near the origin. From the complex inverse mapping on the extended complex plane, we know that the line in the complex $\frac{1}{\beta}$ plane corresponds to a circle in the complex $\beta$ plane. The accumulation in the complex $\frac{1}{\beta}$ plane means there is no zero 


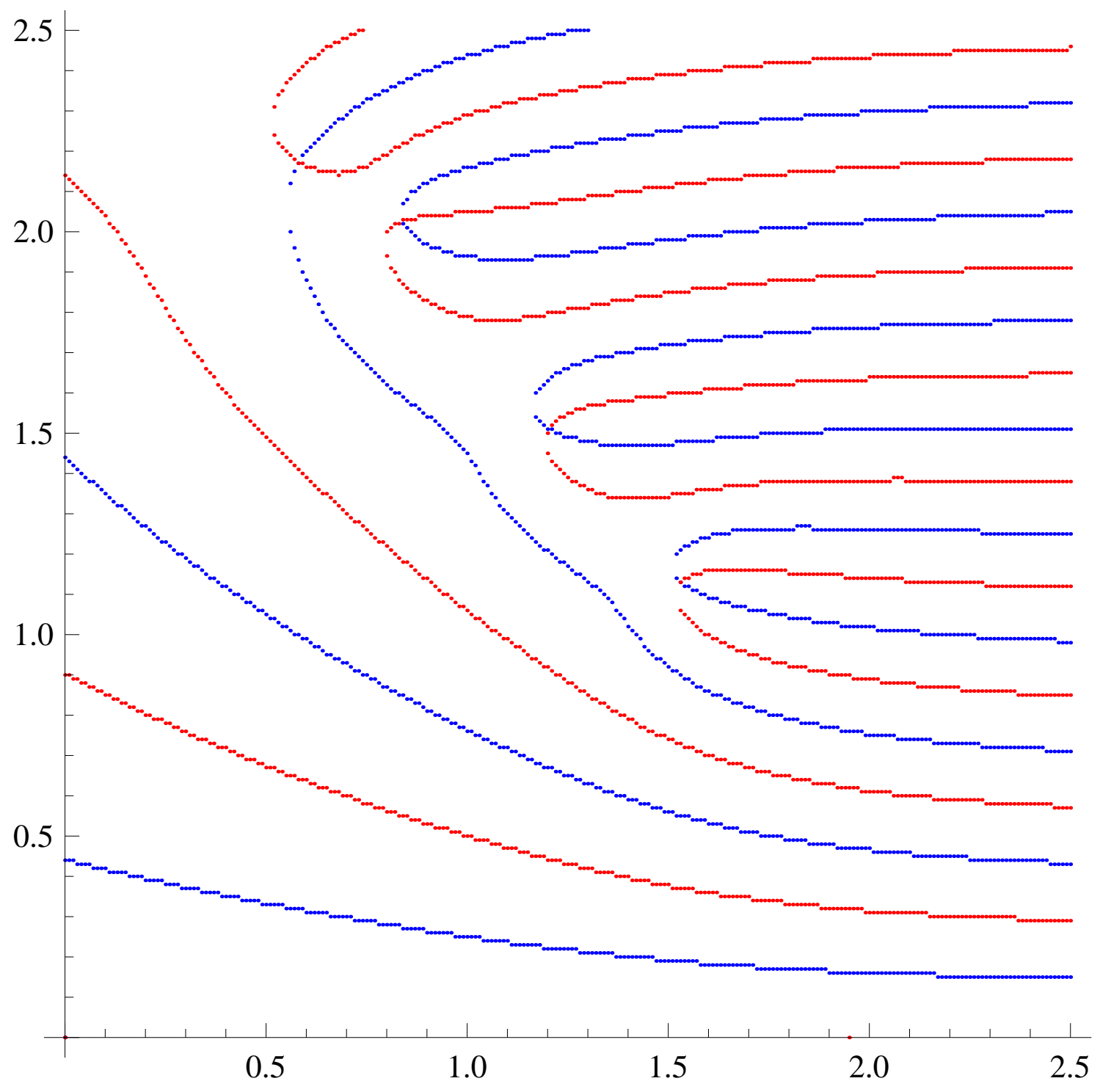

Figure 2.1: The partition zeros in the complex $\beta$ plane for the HM with volume $2^{4}$ in 3 dimension. 


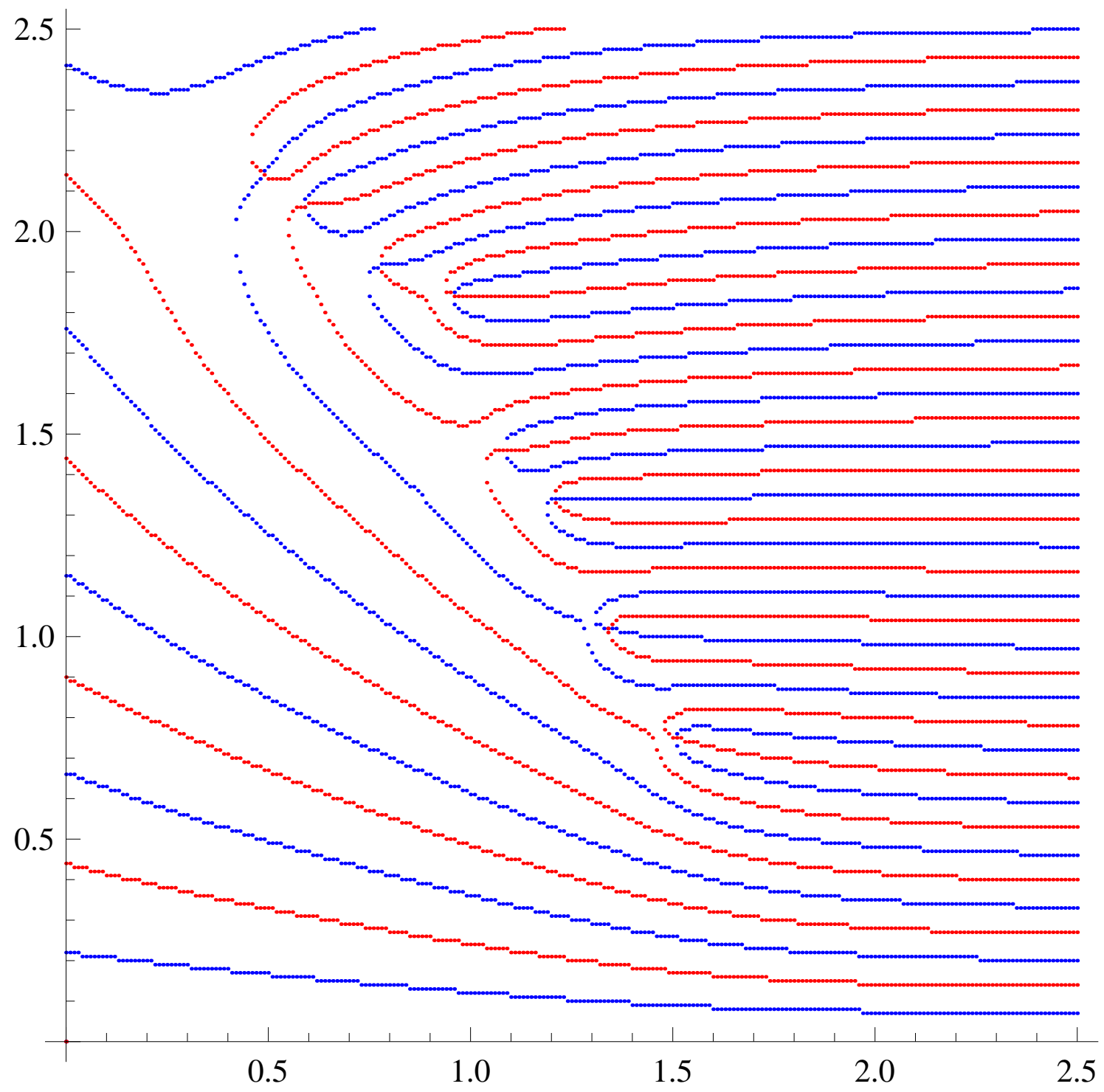

Figure 2.2: The partition zeros in the complex $\beta$ plane for the HM with volume $2^{5}$ in 3 dimension. 


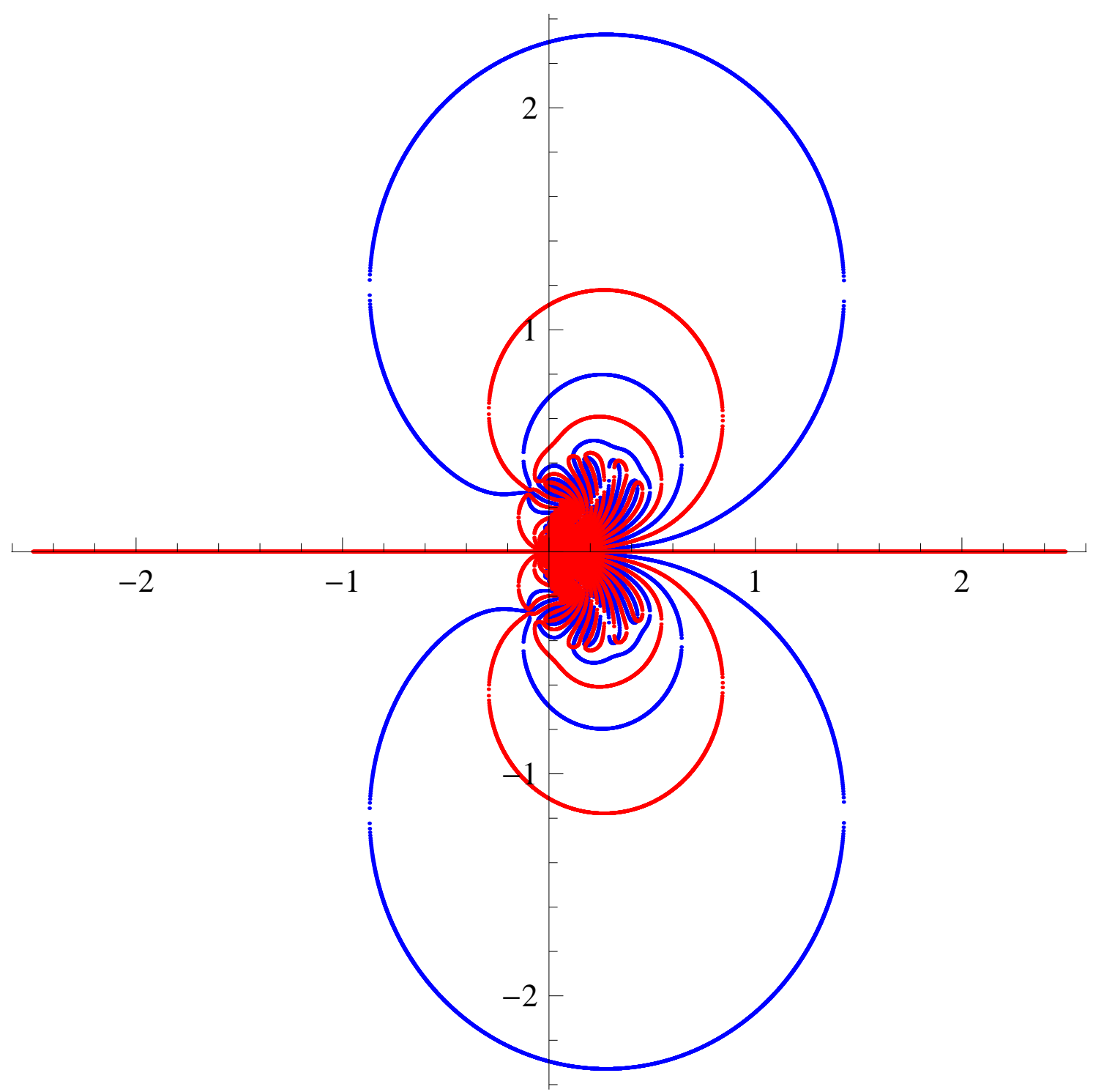

Figure 2.3: Same system as in Fig. 2.1 but in the complex inverse $\beta$ plane.

near the origin in the complex $\beta$ plane. We can also see the corresponding zeros of Figure 2.1 from the Figure 2.4.

\subsubsection{Distribution Of The Zeros For Different Variables}

At the end of this section, we want to briefly discuss the distribution of the Fisher zeros. From figure 2.14, we can clearly see that the number of zeros in the 


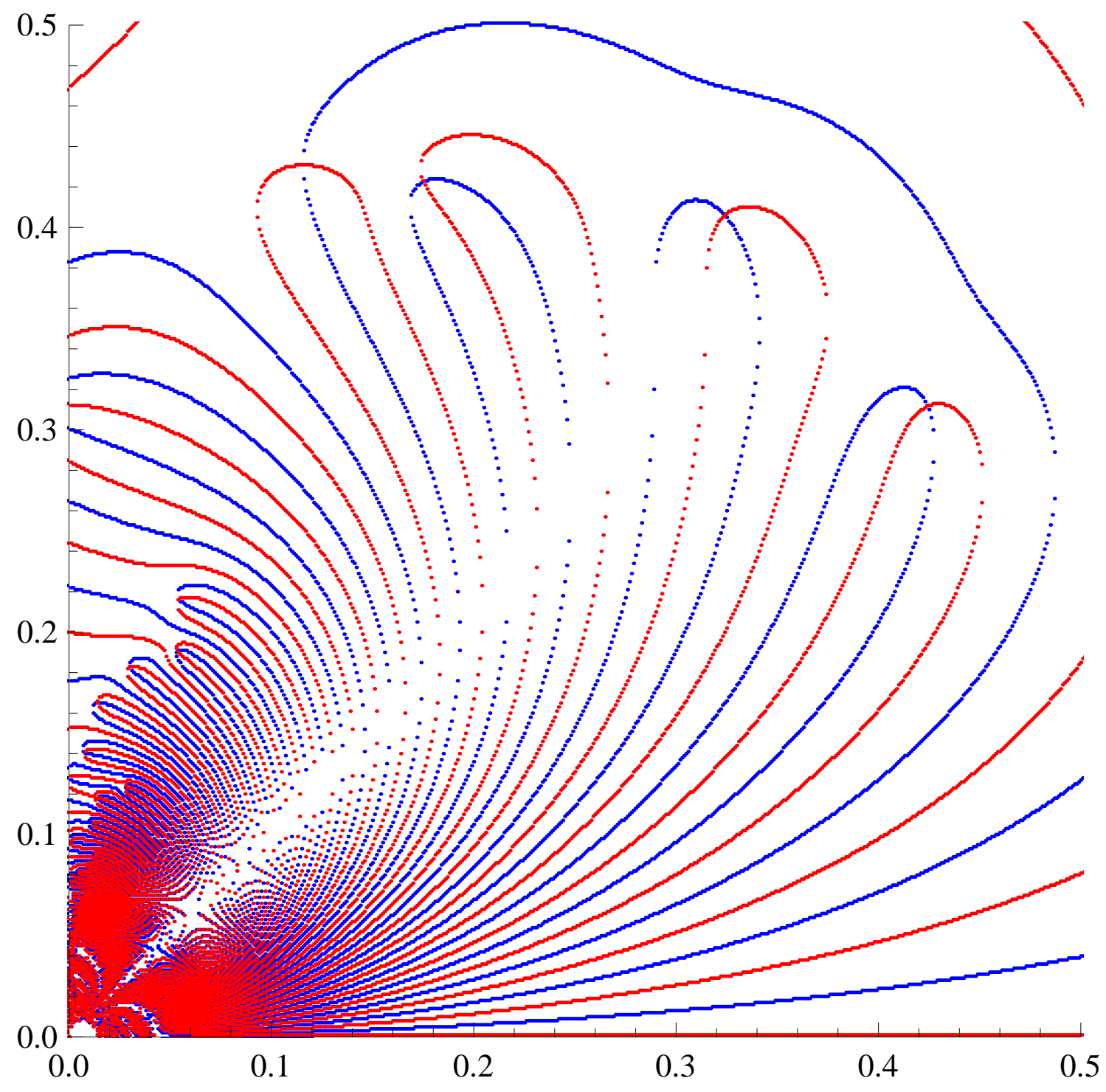

Figure 2.4: Same system as in Fig. 2.1 but in the complex inverse $\beta$ plane. 
specific region is proportional to the logarithm of the volume.

Sometimes the distribution of the partition function zeros is considered in the complex tanh $\beta$ plane or the complex $e^{-2 \beta}$ plane. The zeros is usually distributed on the circles. In the following, we will try to connect different distribution patterns with different choices of variables.

Suppose the Fisher zeros in the complex $\beta$ plane has the form $\beta=\beta_{r}+\mathrm{i} \beta_{i}$. Then if we express these zeros in the complex $\tanh \beta$ plane, we will get

$$
\begin{aligned}
\tanh \beta & =\tanh \left(\beta_{r}+\mathrm{i} \beta_{i}\right) \\
& =\frac{\sinh \left(2 \beta_{r}\right)}{\cos \left(2 \beta_{i}\right)+\cosh \left(2 \beta_{r}\right)}+\mathrm{i} \frac{\sin \left(2 \beta_{i}\right)}{\cos \left(2 \beta_{i}\right)+\cosh \left(2 \beta_{r}\right)} .
\end{aligned}
$$

We further assume $\beta_{r}$ is a constant, which was observed in the hierarchical Ising model in the infinite volume limit. That means the distribution of the Fisher zeros form a line which is parallel to the imaginary $\beta$ plane (Actually there are two lines, one crosses the positive $\beta$ and another crosses the negative $\beta$. We are only interested in the real $\beta$ or ferromagnetic region.). Then in the $e^{-2 \beta}$ or tanh $\beta$ plane, the Fisher zeros will distribute in a circle. The reason is that both $f(\beta)=e^{-2 \beta}$ and $f(\beta)=\tanh \beta$ are conformal mapping and they will map the lines parallel to the imaginary axis in the $\beta$ plane to circles in the $\beta$ and $\tanh \beta$ plane.

The following are examples of the distributions of zeros in different variables. The partition function for $D=2$ and $V=2^{3} \mathrm{HM}$ is

$$
\begin{aligned}
Z(\beta)= & 64 e^{21 \beta / 32}+16 e^{\beta}+32 e^{5 \beta / 4}+32 e^{11 \beta / 8} \\
& +16 e^{13 \beta / 8}+32 e^{53 \beta / 32}+4 e^{2 \beta}+16 e^{69 \beta / 32}+ \\
& 16 e^{77 \beta / 32}+8 e^{21 \beta / 8}+2 e^{3 \beta}+2 e^{7 \beta / 2}+16 .
\end{aligned}
$$

It is easy to change the variable from $e^{\beta}$ to $x^{3} 2$ and then write the partition as a polynomial of variable $x$. We can then get all the roots of the polynomial by 
conventional methods. Figure 2.6 is a plot of all the roots in the complex $x=e^{\beta / 32}$ plane for $V=2^{4}$ case. We can see "clear" pattern that all the roots are located around the unit circle. However, if we plot the roots in the complex $\log (x)$ plane, then the roots will scatter around the plane and no obvious pattern persists.

Things become more difficult when we switch to $D=3$ case. The following is the partition function for $D=3$ and $V=2^{3}$,

$$
\begin{aligned}
& Z(\beta)=16 e^{\sqrt[3]{2} \beta}+4 e^{2 \sqrt[3]{2} \beta}+64 e^{\frac{\beta}{4 \sqrt[3]{2}}+\frac{\beta}{2^{2 / 3}}+\frac{\beta}{16}} \\
& +32 e^{\sqrt[3]{2} \beta+\frac{\beta}{4 \sqrt[3]{2}}+\frac{\beta}{2^{2 / 3}}+\frac{\beta}{16}} \\
& +32 e^{\sqrt[3]{2} \beta+\frac{\beta}{2 \sqrt[3]{2}}}+32 e^{\sqrt[3]{2} \beta+\frac{\beta}{2 \sqrt[3]{2}}+\frac{\beta}{4}} \\
& +16 e^{\sqrt[3]{2} \beta+\frac{\beta}{\sqrt[3]{2}}+\frac{\beta}{4}}+16 e^{\sqrt[3]{2} \beta+\frac{5 \beta}{4 \sqrt[3]{2}}+\frac{\beta}{2^{2 / 3}}+\frac{\beta}{16}} \\
& +16 e^{\sqrt[3]{2} \beta+\frac{5 \beta}{4 \sqrt[3]{2}}+\frac{\beta}{2^{2 / 3}}+\frac{9 \beta}{16}}+8 e^{2 \sqrt[3]{2} \beta+\frac{\beta}{\sqrt[3]{2}}+\frac{\beta}{4}} \\
& +2 e^{2^{2 / 3} \beta+2 \sqrt[3]{2} \beta}+2 e^{2^{2 / 3} \beta+2 \sqrt[3]{2} \beta+\beta}+16 .
\end{aligned}
$$

\subsection{6 $\quad D=2$}

In Ref. [123], we try to argue that the Fisher zeros form the "gate" to control the RG flows. In the following, we will consider two different cases: the first case has no phase transition and the other one has a second order phase transition. We will see how we can extract the information about the phase transition from the location of the Fisher zeros.

In the $D=2$ case, the HM has no phase transition at finite temperature [82]. That means that the partition function should not be zero on the real axis even in the infinite volume limit. In figure (2.7) we plot the lowest Fisher zeros of the HM with different volume. The distribution of the Fisher zeros is symmetric about the real $\beta$ plane so that we only need to show the zeros in the upper half plane. 


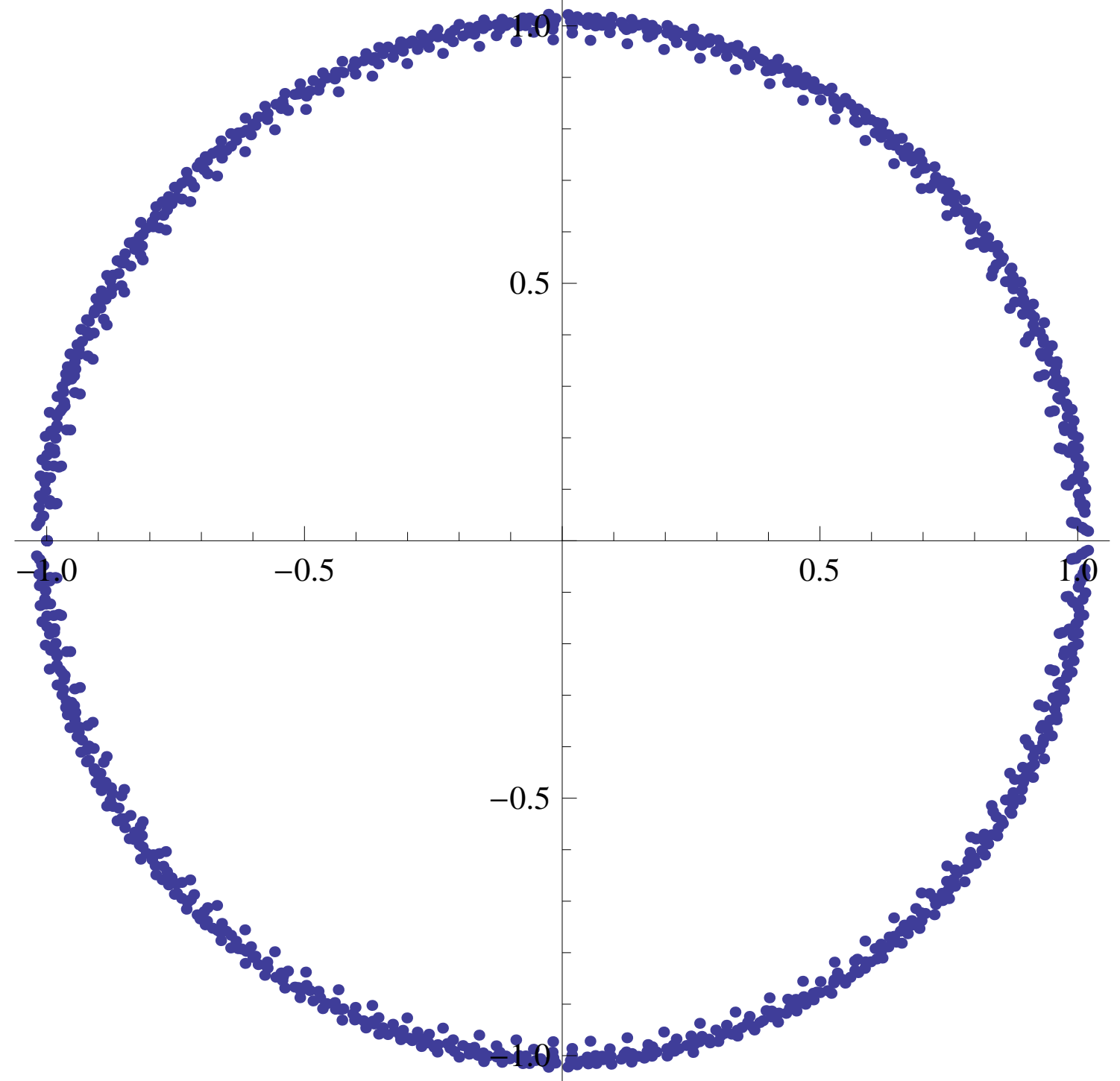

Figure 2.5: The roots of the hierarchical model partition for $D=2$ and $V=2^{4}$ in the $x=e^{\beta}$ plane. 


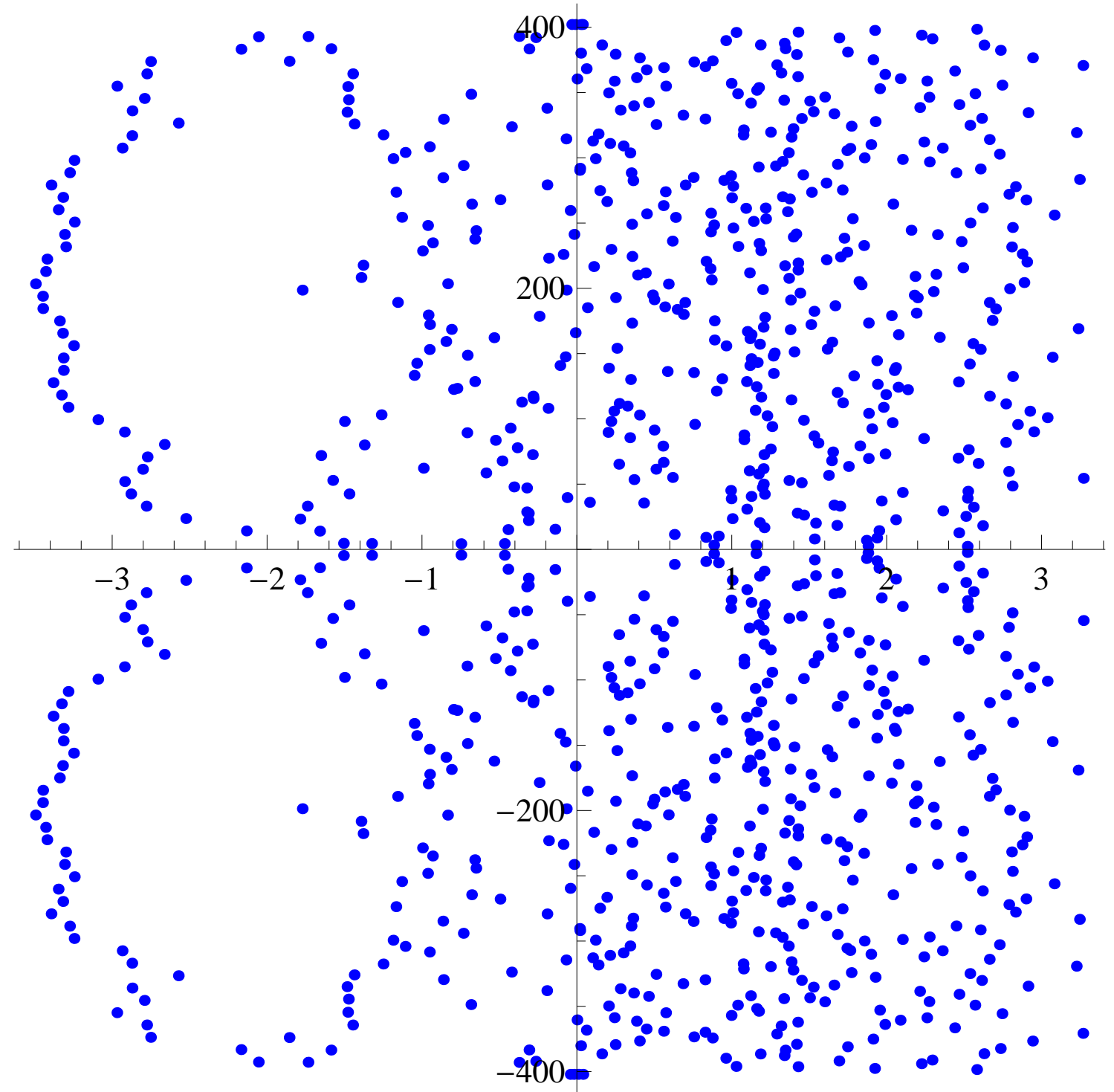

Figure 2.6: The roots of the HM partition for $D=2$ and $V=2^{4}$ in the $\log (x)=$ $\beta / 128$ plane. 


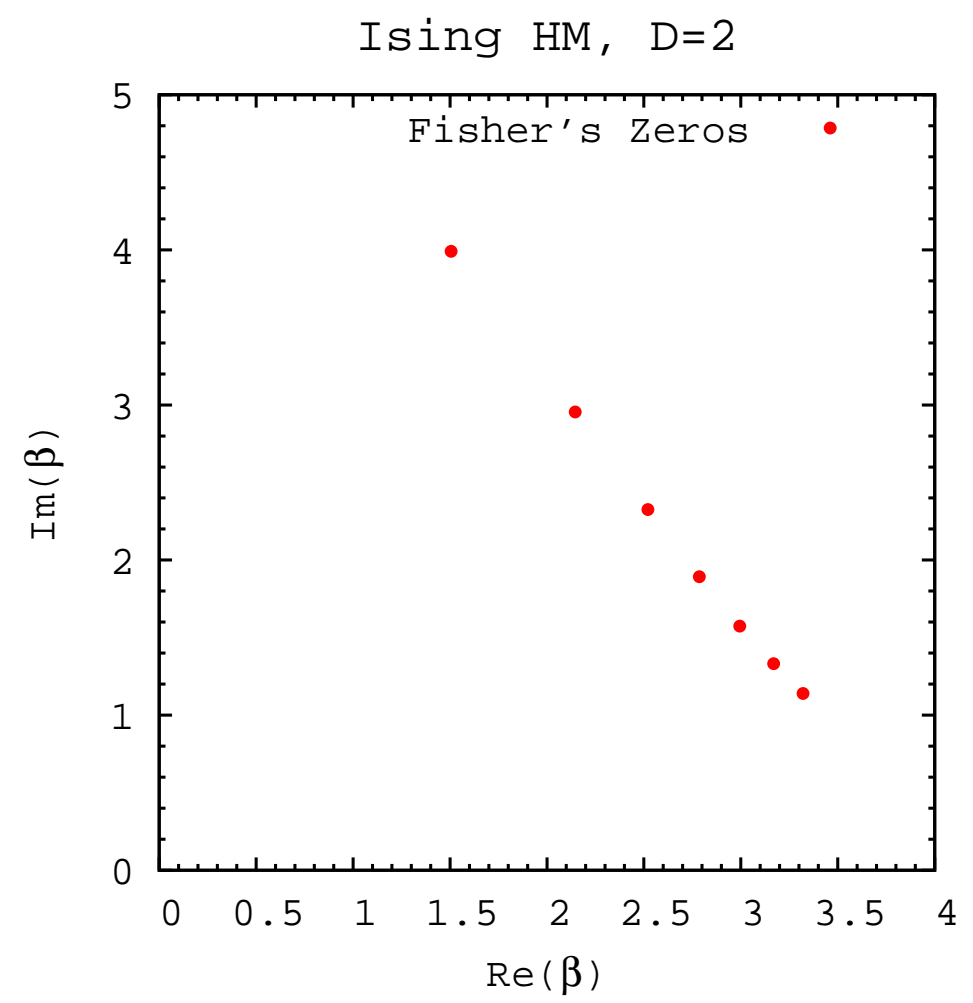

Figure 2.7: The Fisher zeros for the two dimensional HM with different volume $V=2^{N}$; from up to down: $N=2,3,4,5,6,7,8$.

The partition function has infinite number of complex zeros due to the periodicity of the exponential function. Here we are only interested in the zero that is closest to the real axis, or the "lowest" zero in the point of view of the absolute value. Because only through these zeros we can decide the order of the phase transition. For $D=2$, the lowest Fisher zeros get closer and closer to the real axis as the volume increases and at the same time, they move to the right with a slower rate. There is no tendency for the zeros to pinch the real axis. In figure (2.8), we plot the real and imaginary part of the lowest zeros with the same data as in Fig. (2.7). 


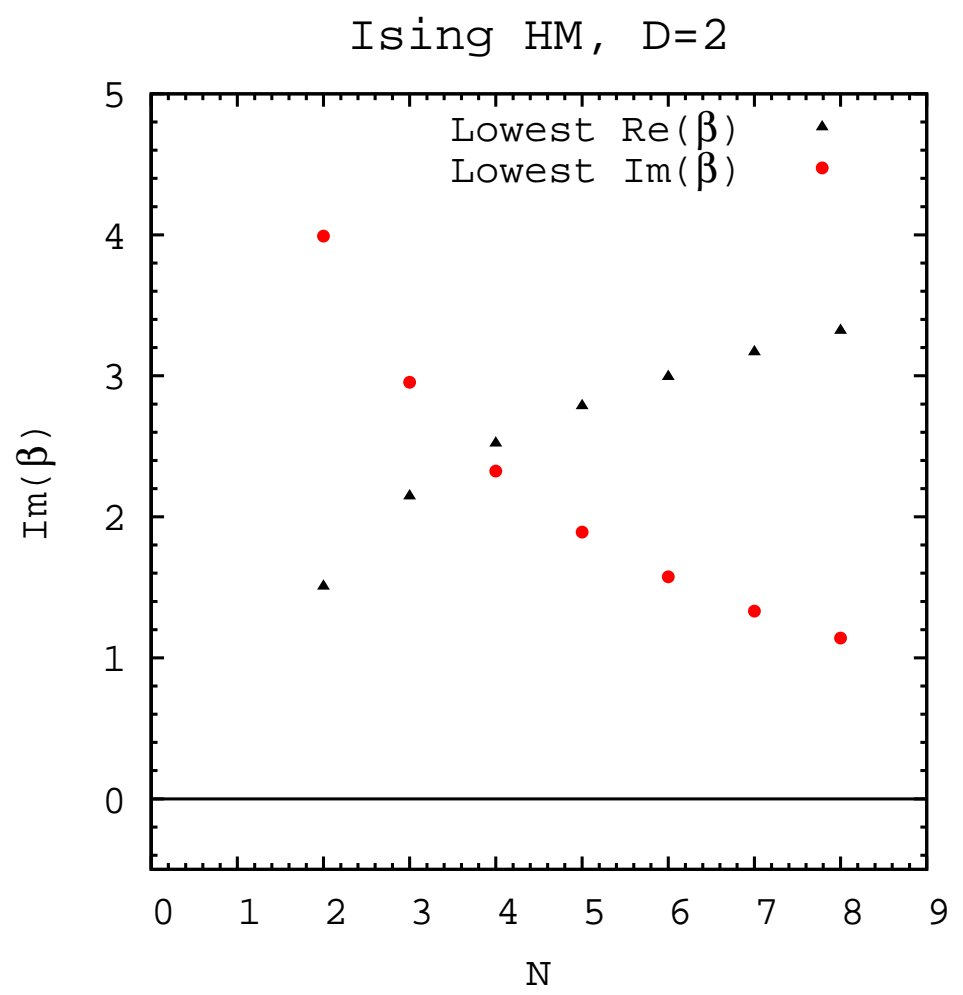

Figure 2.8: The real and imaginary part of the Fisher zeros for the two dimensional HM with different volume $V=2^{N}$. 


\section{$2.2 .7 \quad D=3$}

For $D=3$ case, the system has a second order phase transition. The second derivative of the free energy diverges at the some particular temperature (or inverse temperature $\beta$ ) in the thermodynamic limit. This temperature is called the critical temperature. The partition function must be zero at the critical temperature. We know that the critical $\beta$ for the three dimensional HM is around 1.179 [118]. In figure (2.9), we can see that the lowest Fisher zeros really accumulate around 1.179 for the large volume and they move faster to the real axis than the two dimensional case. Another interesting phenomena can be observed if we compare Fig. 2.7 with Fig. 2.9 is that the lowest zeros go all the way to the right for the two dimensional case but they curve back to the critical $\beta$ for the three dimensional case. That is a characteristic to distinguish the order of the phase transition. That means that we might only need to calculate the lowest Fisher zeros for several small volumes and "guess" the order of the phase transition by looking at the tendency of the zeros to move. Figure 2.10 again is the real and imaginary part of the lowest zeros with the same data as in Fig. 2.9.

\subsubsection{Finite Size Scaling}

Up to now, we only have Fisher zeros up the volume $V=2^{11}$, which is still a small system compared to the Avogadro constant $2^{23}$. For a system with a second order phase transition, the phase transition only occurs in the thermodynamic limit, in which the volume $(V)$ and the number of $\operatorname{particles}(N)$ are infinite while the density $\rho=V / N$ stays finite $[124,125]$. For the finite volume system, the intensive quantities are continuous functions of the temperature $(T)$, magnetic field $(H)$, etc. That means that there is no true critical point for a finite system. While in the 


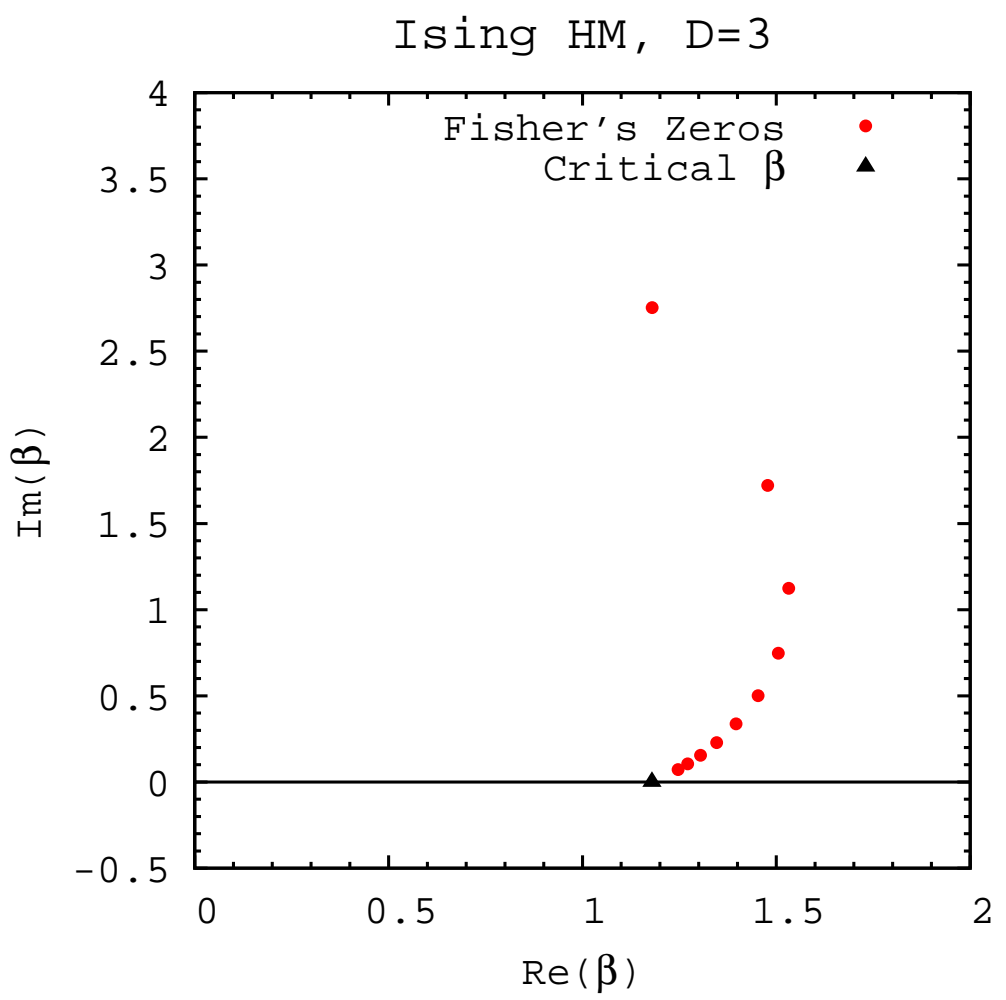

Figure 2.9: The Fisher zeros and the critical $\beta$ for the 3 dimensional HM with different volume $V=2^{N}$; from up to down: $\mathrm{N}=2,3,4,5,6,7,8,9,10,11$. 


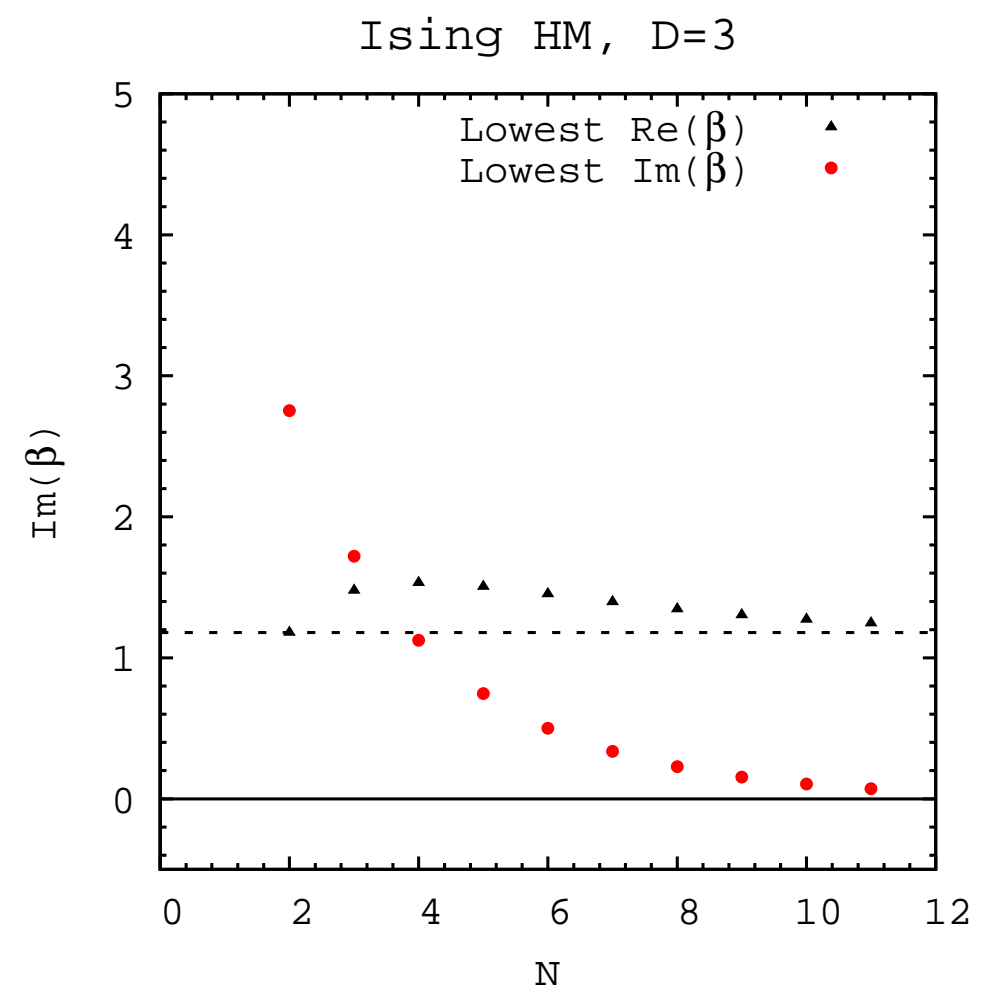

Figure 2.10: The real and imaginary part of the Fisher zeros for the 3 dimensional HM with different volume $V=2^{N}$. 
thermodynamic limit, the divergence may occur. In order to obtain the thermodynamic limit information from the finite volume system, we will need finite size scaling (FSS) technique.

In finite size system, there are three length scales: $a, L$ and $\xi$. Near the critical point, $a$ dependence is very weak. The thermodynamic quantities depend on $\frac{\xi}{L}$. We can treat $L^{-1}$ as a scaling variable, with eigenvalue $y=1$. The FSS assumes that: $<O>_{L}=L^{\alpha / \nu} \phi\left(L^{\frac{1}{\nu}} t\right)$ if $<O>_{\infty}(t) \propto|t|^{-\alpha}$ when $t \rightarrow \infty$, where $t=\left(T-T_{c}\right) / T_{c}$. The above relation can be proved by using renormalization group (RG) transformation.

Itzykson et al. [74] pointed out that the leading zeros $\left(\beta_{1}(L)\right)$ of the partition function obey the finite size scaling relation. One could extract the critical exponents from the leading zeros. More precisely, one would have

$$
\beta_{1}(L)-\beta_{c} \approx A L^{-\frac{1}{\nu}}
$$

where $L=V^{\frac{1}{D}}$ is the linear size of the system and $\nu$ is the critical exponent associated with the correlation length. We note that $L$ here might not be integers in the system studied, although the total number of the sites $V$ is always integer. For example, we could have $V=2^{2}, D=3$ and $L=V^{\frac{1}{D}}=4^{\frac{1}{3}}$. As mentioned above, the critical temperature $\beta_{c}$ has been known for a while [118]: $\beta_{c}=1.17903017 \cdots$ and in principle could be calculated to any significant digits. If the relation 2.46 holds, it is straight forward to get the critical exponent $\nu$ by performing a linear fit of the leading zeros(See Fig. 2.11). Comparing with the known value $\nu=$ $0.64957[126,127]$, the critical exponent we extracted from the Fisher zeros is $1.3 \%$ larger. It has been suggested that the scaling of the imaginary part of the Fisher zeros corresponds to the critical exponent $\nu$ and the real part of the lowest zeros corresponds to the shift exponent $\lambda$ [128], where the shift exponent is defined as $\beta_{L}-\beta_{c} \mid \approx L^{-\lambda}$. However, we found out that the distance scaling, where the 


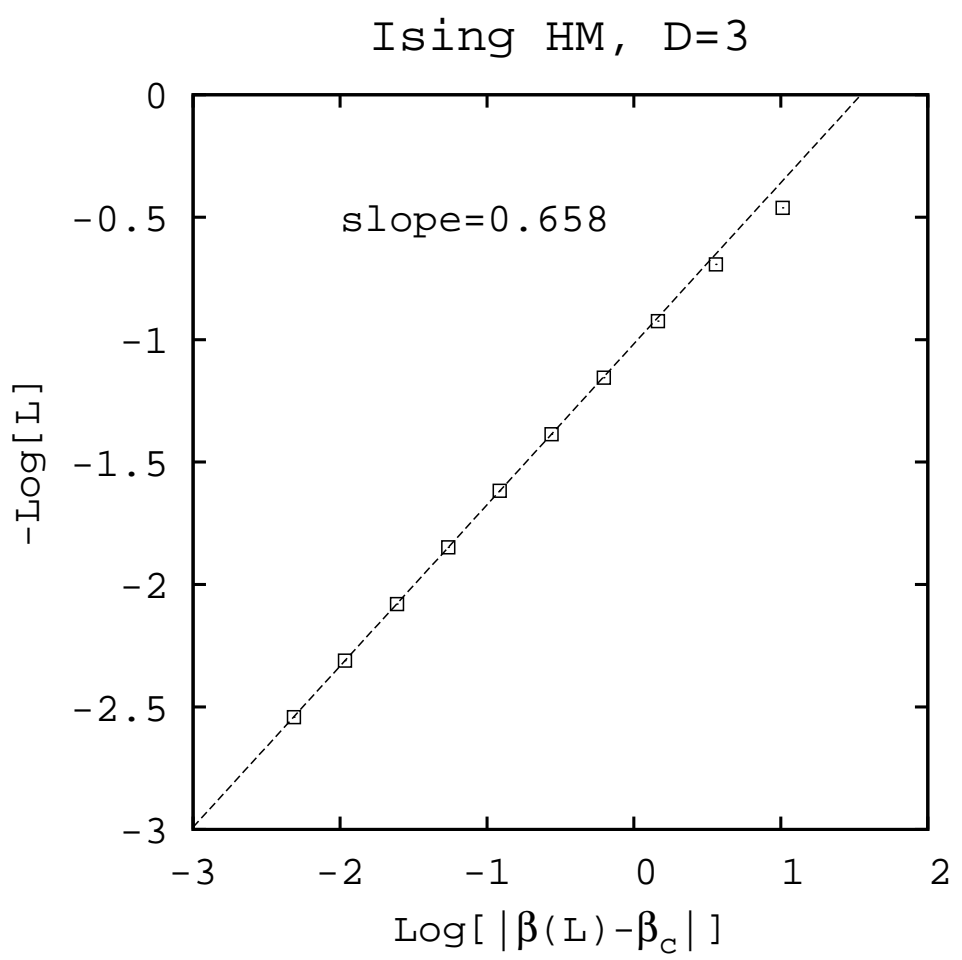

Figure 2.11: $-\ln L$ versus $\ln \left|\beta(L)-\beta_{c}\right|$ for $n=2-11$; the fitting was done with $n=8-11$.

information of both the real and imaginary part of the zeros is used, is optimum for the extraction of the critical exponent $\nu$. See Fig. 2.12 and 2.13 for the scaling of the imaginary and real part of the zeros. We notice that the exponent extracted from the imaginary part of the zeros is smaller than $\nu$ while the corresponding real part is larger.

Following Itzykson et al. [74], we could also build up the relationship between the zeros of the partition function and the sub-leading critical exponent $\omega$. We know that the zeros of the partition function is the same as the zeros of the scaling function $Q$

$$
Z_{1 / \lambda}\left(K_{1} L^{y_{1}} \lambda^{y_{1}}, \cdots\right)=Q\left(x_{1}, x_{2}, \cdots\right)
$$




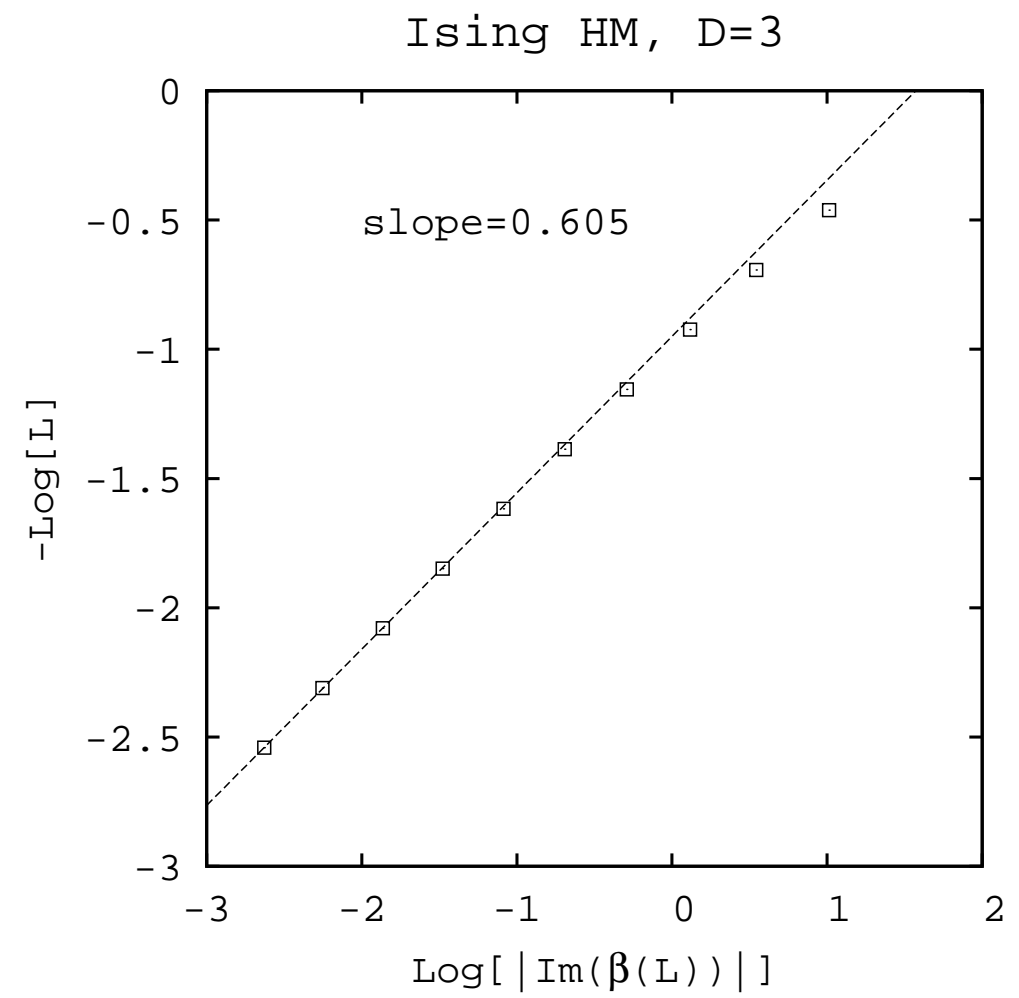

Figure 2.12: $-\ln L$ versus $\ln |\operatorname{Im} \beta(L)|$ for $n=2-11$; the fitting was done with $n=8-11$. 


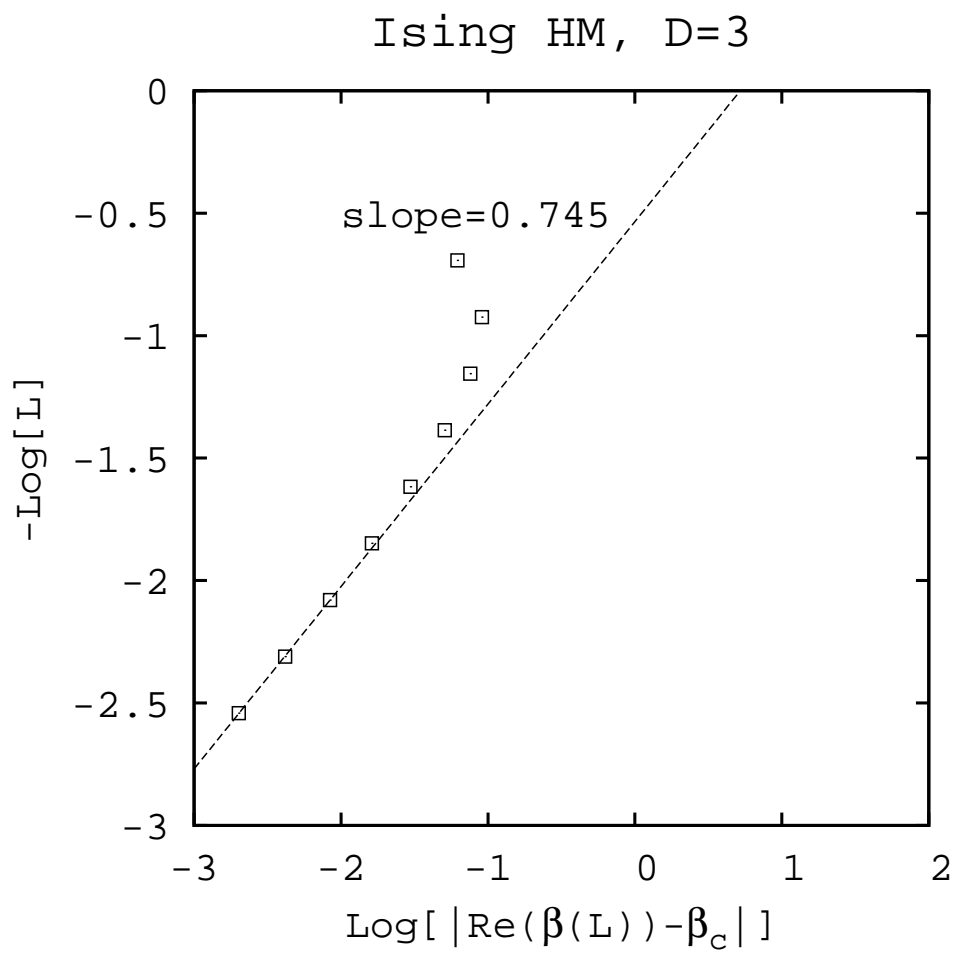

Figure 2.13: $-\ln L$ versus $\ln \left|\operatorname{Re} \beta(L)-\beta_{c}\right|$ for $n=3-11$; the fitting was done with $n=8-11$. 


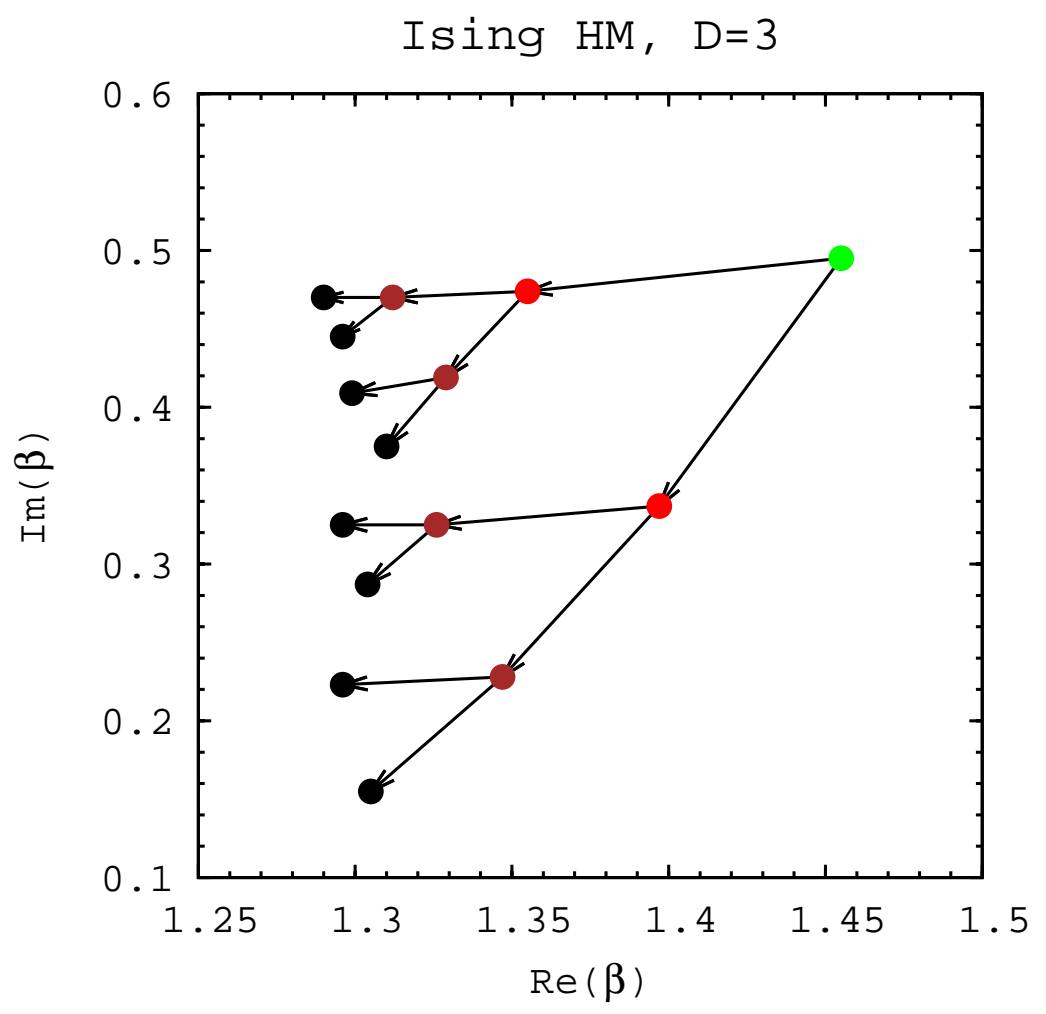

Figure 2.14: The density of the zeros is proportional to $\ln V$. 
where $\lambda=L / b$ and $x_{i}=K_{i} L^{y_{i}}$ are the scaling variables. Here we will consider the zero field case $(h=0)$. In this case, if we consider the leading thermal scaling variable and the sub-leading variable, we will have the following scaling relation for the zeros of the $Q$

$$
Q\left(x_{t}, x_{\omega}\right)=Q\left(K L^{1 / \nu}, U L^{-\omega}\right)
$$

where $K=\beta-\beta_{c}$ and $\omega$ corresponds to the exponent of the sub-leading correction. We further assume that we could solve the above equation and get

$$
K L^{1 / \nu}=f^{-1}\left(U L^{-\omega}\right),
$$

If we expand the right hand side of the equation 2.49 and only keep the linear term in $L^{-\omega}$, we will get

$$
\begin{aligned}
K L^{1 / \nu} & =\left(\beta-\beta_{c}\right) L^{1 / \nu} \\
& =f^{-1}\left(U L^{-\omega}\right) \\
& =A+B L^{-\omega}+O\left(L^{-2 \omega}\right)
\end{aligned}
$$

where $A$ and $B$ are in general both complex numbers. Notice that we have absorbed the coefficient $U$ into $B$.

From equation 2.52 , we can get the scaling relation of both real and imaginary part of the partition function zeros

$$
\begin{array}{r}
\operatorname{Re}(\beta(L))-\beta_{c}=\operatorname{Re}(A) L^{-1 / \nu}+\operatorname{Re}(B) L^{-1 / \nu-\omega} \\
\operatorname{Im}(\beta(L))=\operatorname{Im}(A) L^{-1 / \nu}+\operatorname{Im}(B) L^{-1 / \nu-\omega} .
\end{array}
$$

We have the values for $\beta_{c}, \nu$, and $\omega$ already so that we could use these values to test the above scaling relations. In figure 2.15, the real and imaginary part of the zeros with volume ranging from $V=2^{2}$ to $V=2^{11}$ are shown along with the fitting curves based on the equation 2.53 and 2.54 .

The fittings agree with the data except for the smallest volume. This opens 
Ising $H M, R e$ and Im of lowest zeros, $D=3$

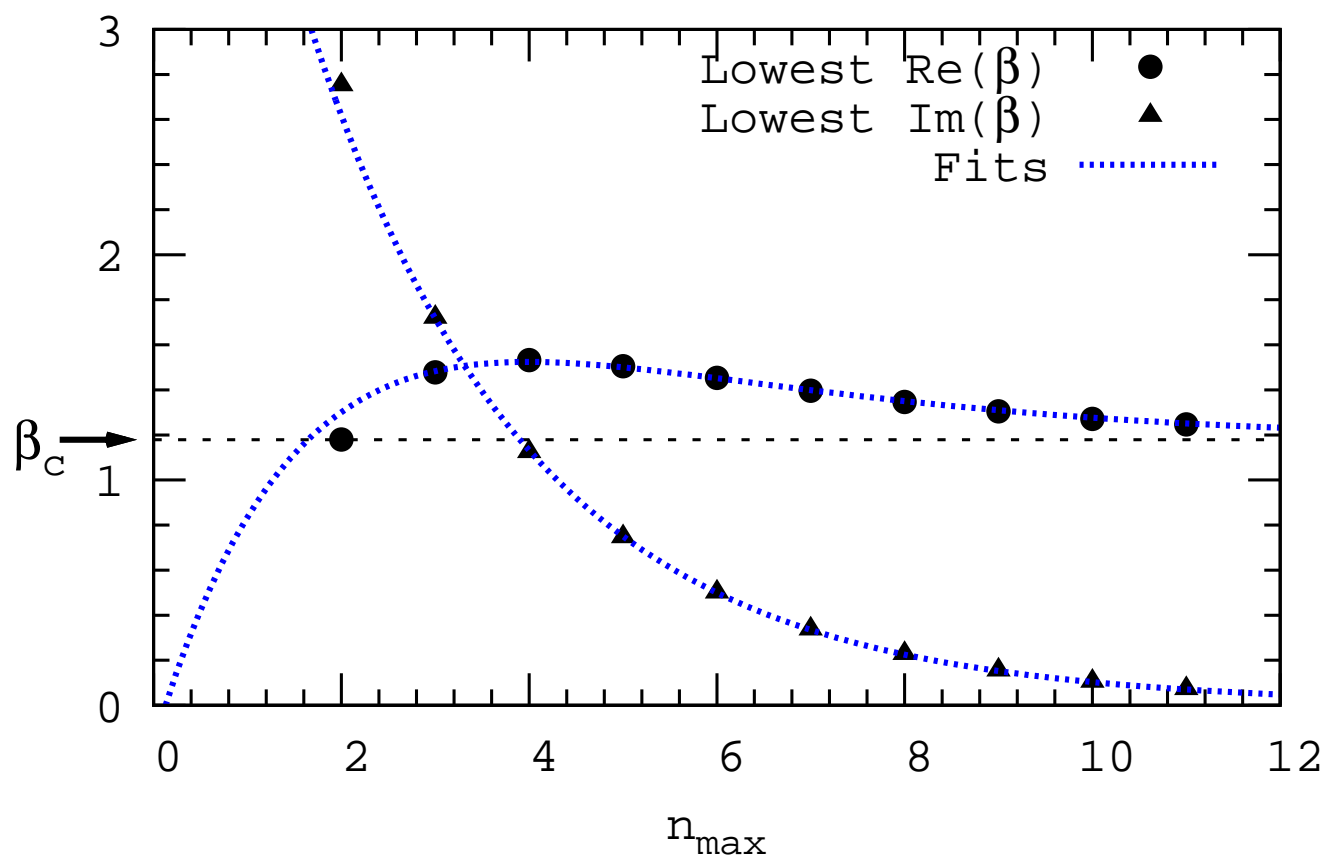

Figure 2.15: The real and imaginary parts of the Fisher zeros for $n=2-11$. 
up the possibility of extracting the sub-leading exponent from the Fisher zeros.

\subsubsection{From Discrete To Continuous RG transformation}

Up to now, we only considered $b^{D}=2$ case. Actually, we are also interested in the limit $b \rightarrow 1$ for the following reasons. In recent years, the local potential approximation (LPA) has been widely used in the context of Exact Renormalization Group Equations (ERGE), see next section, and has generated a lot of interest. We recommend references $[129,130]$ for reviews of the recent progress. ERGE allow in principle the study of global aspects of the RG flows of field theoretical or statistical models. However, truncation methods are necessary in order to make practical calculations. One particularly popular choice is to combine Polchinski's ERGE [131] with the LPA [132]. This results in a simple partial differential equation (called the "Polchinski's equation" below) for the effective potential. Polchinski's equation can also be obtained [133] as an infinitesimal version of the RG equation for the HM. This suggests that the linearized theories should be close to each other. Many accurate calculations of the critical exponents $[134,135,136,137,138]$ show that the exponents differ only in the fifth significant digit. This small difference is significant. We find that the critical exponents of the HM depends on the blocking scheme. There is also a logarithmic term preventing one going from discrete RG transformation to the continuous one. These will be shown in section 2.2.10 and 2.2.11.

For $b=2$ and $D=3$, the value $\gamma=1.30033 \ldots$ was obtained numerically in Ref. [139]. This value is significantly different from the value $\gamma=1.2991407 \ldots$ obtained in references $[126,127]$ for $b=2^{1 / 3}$ and $D=3$. For $D=3$, the limit $b \rightarrow 1$ was studied in Ref. [140] with the result $\gamma=1.299124$. The difference in the fifth digit is significant and was confirmed by new calculations [138]. This shows that again different values of $b$ correspond to different classes of universality and also 
that for a given $b \neq 2^{1 / D}$, the extensions given in equations are in-equivalent. In addition we see that the $b$ dependence is much weaker and the slope is apparently opposite to the slope found analytically. We calculated the the critical exponent $\gamma$ for integer $b^{D}$ up to 8. We try to extend the calculation to non-integer $b^{D}$. However, we found there is a stability window where everything converges only within some window of $l_{\max }$ (see section 2.2.11 for definition of $l_{\max }$ ). This prevents us from making connections between discrete block spinning(integer $b^{D}$ ) transformation and the continuous one $\left(b^{D} \rightarrow 1\right)$.

\subsubsection{Linear Analysis}

\subsubsection{Fixed Point Equation}

Usually we normalize the recursion formula (2.38) by requiring the constant term in $R_{n}(k)$ to be 1 in order for $R_{n}(k)$ to have a direct probabilistic interpretation. In this case, the fixed point equation is :

$$
R^{*}(k)=C_{n+1} \mathrm{e}^{-\frac{1}{2} \beta_{c} \frac{\partial^{2}}{\partial k^{2}}}\left(R^{*}\left(b^{-(D+2) / 2} k\right)\right)^{b^{D}} .
$$

We can get the stability matrix $L$ by linearizing the RG transformation right about the fixed point $R^{*}$. In the above case, we will only get one eigenvalue that is larger than 1 which corresponds to the relevant direction. The critical exponent $\gamma$ can be obtained from $\gamma=\ln (2 / c) / \ln \lambda_{1}$, where $\lambda_{1}$ is the largest eigenvalue.

However, we can also absorb the normalization coefficient into the $R^{*}(k)$ by multiplying both sides of (2.55) by $C_{n+1}^{1 /\left(b^{D}-1\right)}$. The new fixed point equation becomes

$$
R_{0}^{*}(k)=\mathrm{e}^{-\frac{\beta_{c}}{2} \frac{\partial^{2}}{\partial k^{2}}}\left(R_{0}^{*}\left(b^{-(D+2) / 2} k\right)\right)^{b^{D}} .
$$

The corresponding polynomial expansion becomes

$$
R_{n}(k)=a_{n, 0}+a_{n, 1} k^{2}+a_{n, 2} k^{4}+\ldots+a_{n, l_{\max }} k^{2 l_{\max }},
$$

or equivalently (only even power of $k$ is involved due to $\mathrm{Z}(2)$ symmetry of the $\phi$ 
field),

$$
R_{n}(u)=a_{n, 0}+a_{n, 1} u+a_{n, 2} u^{2}+\ldots+a_{n, l_{\max }} u^{l_{\max }} .
$$

The ${ }_{0}$ under the $R^{*}$ in (2.56)means the 0th order in the following small $\zeta$ expansion about integer $b^{D}$. If we use the above unnormalized fixed point to get the stability matrix, we will get two eigenvalues larger than 1. The first one is just $\lambda_{0}=b^{D}$. The reason we use the unnormalized fixed point equation is that we do not need to expand the coefficient $C_{n+1}$ when we do the small $\zeta$ expansion which considerably simplifies the calculation.

\subsubsection{Stability Matrix}

Let us make a small variation $\delta R$ around fixed point $R^{*}$

$$
R_{0}^{*}+\delta R^{\prime}=R G_{0}\left[R_{0}^{*}+\delta R\right]=R_{0}^{*}+L[\delta R]
$$

where $R G_{0}$ stands for the 0th order RG transformation. If we only keep the first order in $\delta R$, we will get the stability matrix $L$

$$
L \approx \mathrm{e}^{-\frac{1}{2} \beta_{c} \frac{\partial^{2}}{\partial k^{2}}}\left[2 R_{0}^{*}\left(b^{-(D+2) / 2} k\right)\right]
$$

In general, the stability matrix is not symmetric and involves very large and very small numbers. Fig.2.16 shows a typical stability matrix. The matrix elements range from $10^{-109}$ to $10^{+42}$ for $l_{\max }=40$. That requires us to use very high precision for the calculations. The non-symmetric property also requires the consideration of the left and right eigenvectors separately (see section 2.2.10.3 for details).

For the special $b^{D}=2$ case, the stability matrix can be calculated in the following way

$$
\begin{aligned}
\delta a_{n+1, \sigma} & =\sum_{\nu=0}^{l_{\max }} L_{\sigma, \nu} \delta a_{n, \nu} \\
L_{\sigma, \nu} & =\frac{\partial a_{n+1, \sigma}}{\partial a_{n, \nu}} \\
a_{n+1, \sigma} & =\Gamma_{\sigma}^{\mu \nu} a_{n, \mu} a_{n, \nu}
\end{aligned}
$$




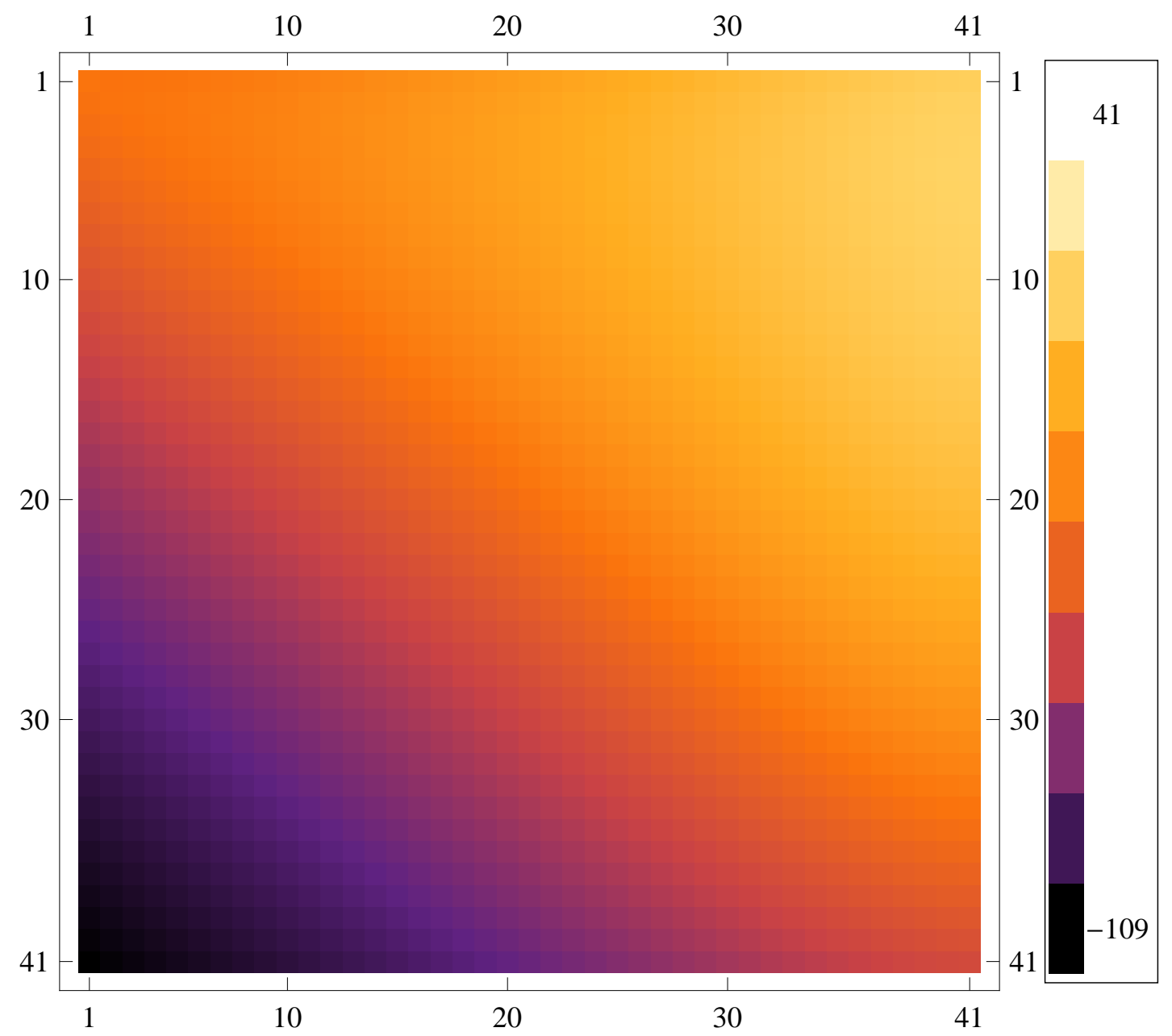

Figure 2.16: Stability matrix for $l_{\max }=40$. 


$$
\Gamma_{\sigma}^{\mu \nu}=(c / 4)^{\mu+\nu} \frac{(-1 / 2)^{\mu+\nu-\sigma}(2(\mu+\nu)) !}{(\mu+\nu-\sigma) !(2 \sigma) !} .
$$

\subsubsection{Small $\zeta$ Expansion}

In order to investigate the non-integer $b^{D}$ behavior of the recursion formula, we expand $b^{D}$ right around integer values. This is similar to the $\epsilon$ expansion extensively used in statistical mechanics and quantum field theory. Often, people expand dimension $D$ around integer values, usually 4 or 2 . It has been proved that this theoretical method is quite useful although there is no significant physical meaning. In the following, we will show that we can do a similar expansion for $b^{D}$ in our system instead of $D$. We will fix the dimension of the system to be $D=3$. The physical meaning of $b^{D}$ in our system is the number of sites blocked in each RG transformation. For example, for the system with $b^{D}=2$ and $D=3$, we block 2 sites in each iteration. One should notice that the "number of sites" blocked in each direction is $2^{1 / 3}$. Blocking non-integer number of sites seems impossible in the position space. However, this is very natural if we consider it in the $k$ space. One should also notice that we not only change the power in the formula (2.56) but also the rescaling factor when we do the $b^{D}$ expansion

$$
\begin{gathered}
b^{D}=2+\zeta, \\
\frac{c}{4}=b^{-(D+2)}=\frac{c_{0}}{4}\left(1-\frac{5}{6} \zeta\right),
\end{gathered}
$$

where $c_{0}$ corresponds to the $\zeta=0$ case. We suppose the first order correction to the fixed point $R_{0}^{*}$ is $R_{1}^{*}$. The new fixed point equation to the first order in $\zeta$ becomes

$$
R G_{\zeta}\left[R_{0}^{*}+\zeta R_{1}^{*}\right]=R_{0}^{*}+\zeta R_{1}^{*}
$$

We can again make a small variation around the new fixed point(set $\delta R=-\zeta R_{1}^{*}$ in equation 2.59)

$$
R G_{\zeta}\left[R_{0}^{*}\right]=R_{0}^{*}+\zeta R_{1}^{*}-\zeta L^{\prime}\left[R_{1}^{*}\right]
$$


In the limit $\zeta \rightarrow 0, L^{\prime}$ approaches $L$,

$$
\frac{R G_{\zeta}\left[R_{0}^{*}\right]-R_{0}^{*}}{\zeta} \rightarrow R_{1}^{*}-L\left[R_{1}^{*}\right]
$$

On the other hand, if we expand the left hand side of equation (2.67) to first order in $\zeta$ and compare with the right hand side, we obtain

$$
R_{1}^{*}=L\left[R_{1}^{*}+G\right]
$$

where

$$
G=-\frac{5}{6} k^{2} \frac{\partial R_{0}^{*}}{\partial k^{2}}+\frac{1}{2} R_{0}^{*} \ln R_{0}^{*}
$$

From (2.69) and (2.70), we obtain

$$
\frac{R G_{\zeta}\left[R_{0}^{*}\right]-R_{0}^{*}}{\zeta} \rightarrow L[G]
$$

in the $\zeta \rightarrow 0$ limit. The above relation has been tested numerically for $b^{D}=2$ with different $l_{\max }$.

\subsubsection{Linearization}

If we can construct $R_{1}^{*}$ numerically, we can construct the fixed point for noninteger $b^{D}$ from $R_{0}^{*}$, which interpolate among the fixed points on Fig. 2.17 for $b^{D}=2, \cdots 8$. One might have already noticed that everything we have done is very similar to the variational method used in Quantum Mechanics. The only difference is that we are dealing with non-Hermitian matrix here. Actually the matrix $L$ is not symmetric (see Fig. 2.16). For the non symmetric matrix, the left and right eigenvectors are not each others transpose. In the following, we will choose the right eigenvectors as the basis to expand $R_{1}^{*}$ and $G$. We will also normalize left and right eigenvectors individually. Suppose the right eigenvectors of $L$ are $v_{n}$ and the corresponding eigenvalues are $\lambda_{n}$. Then we can write

$$
\begin{aligned}
& R_{1}^{*}=\sum \alpha_{n} v_{n} \\
& G=\sum \beta_{n} v_{n} .
\end{aligned}
$$


Fixed Point $R_{0}{ }^{*}$
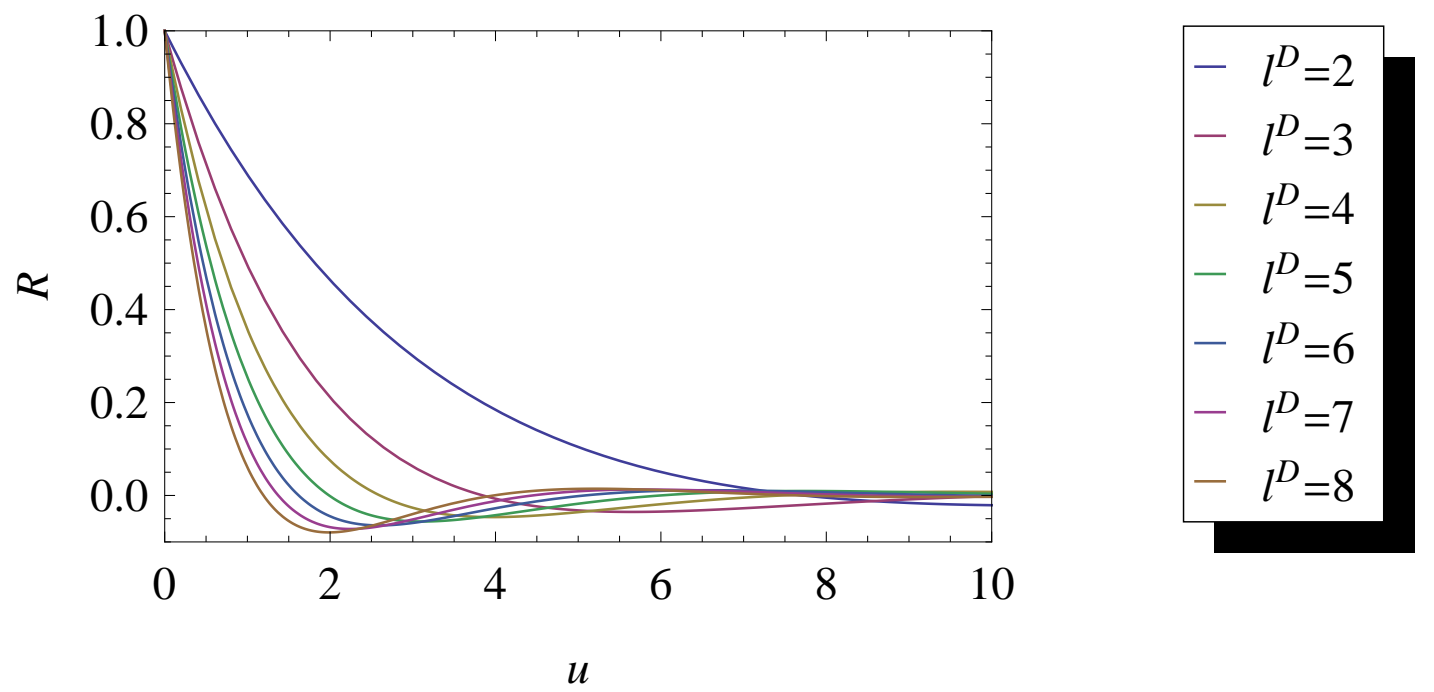

Figure 2.17: The fixed point polynomial $R_{0}^{*}$ for different $b^{D}$.

By using the orthogonality relations among the left and right eigenvectors, we multiply the left eigenvectors on both sides of equation (2.70) and then normalize. We obtain

$$
\alpha_{n}=\frac{\beta_{n} \lambda_{n}}{1-\lambda_{n}}
$$

We have already have $\mathrm{G}$ (see equation $(2.71)$ ). We can easily get $\beta_{n}$ by multiplying both sides of equation (2.74) by the corresponding left eigenvectors. Then $\alpha_{n}\left(R_{1}^{*}\right)$ is obtained from equation (2.75).

\subsubsection{Window Of Stability}

From Eq. 2.71 and 2.74, we obtain

$$
G=-\frac{5}{6} k^{2} \frac{\partial R_{0}^{*}}{\partial k^{2}}+\frac{1}{2} R_{0}^{*} \ln R_{0}^{*}=\sum \beta_{n} v_{n} .
$$

Therefore,

$$
\beta_{n}=\frac{<v_{L} \mid G>}{<v_{L} \mid v_{R}>}
$$

where $v_{L n}$ and $v_{R n}$ are the left and right eigenvectors of $L$. 
In order for $\beta_{n}$ to be stable with increasing $l_{\max }$ (although there is no physical reason to require $\beta_{n}$ to be stable, we should at least avoid singularity.), we require both $<v_{L} \mid G>$ and $<v_{L} \mid v_{R}>$ decrease or increase with the same rate as we increase $l_{\max }$. However, this is not the case. We observe that when we increase $l_{\max }<v_{L} \mid G>$ and $\left\langle v_{L}\right| v_{R}>$ are only parallel within some range. There is a maximum $l_{\max }$ where everything is normal within this range. If we further increase $l_{\max },\left\langle v_{L}\right| G>$ decreases much slower than $\left\langle v_{L}\right| v_{R}>$. See Fig. 2.18 for details. That is to say, when we increase $l_{\max }$, which in principle is equivalent to getting better approximation to the original measure, we will inevitably get very large (if not infinity) $\beta_{n}$. That prevents us from getting accurate critical temperature and critical exponents. We also notice that the stability window is smaller with smaller $b^{D}$. The reason for the diverging property of $\beta_{n}$ is that there is a logarithm term $\ln R_{0}^{*}$ in the expression $G$. The right eigenvectors are not good basis for this logarithm term.

In Fig. 2.19, we show the result $\beta_{c}$ obtained from different $b^{D}$. The red dots correspond to the values for integer $b^{D}$. We can calculate them up to arbitrary precision, which is equivalent to say that we can use arbitrary $l_{\max }$ to approximate the initial measure. The blue dots are for non-integer $b^{D}$. The results for non-integer $b^{D}$ are unstable. That confirms our analysis above.

In Fig. 2.20, the critical exponent $\gamma$ is showed with different $b^{D}$ and $l_{\max }$. All the calculations were performed with maximum $l_{\max }$ in the stability window. We can see that the stability window decreases with decreasing $b^{D}$. The $\gamma$ we can get for non-integer $b^{D}$ can not reach the accuracy acquired for our comparison with LPA (5th digit). 


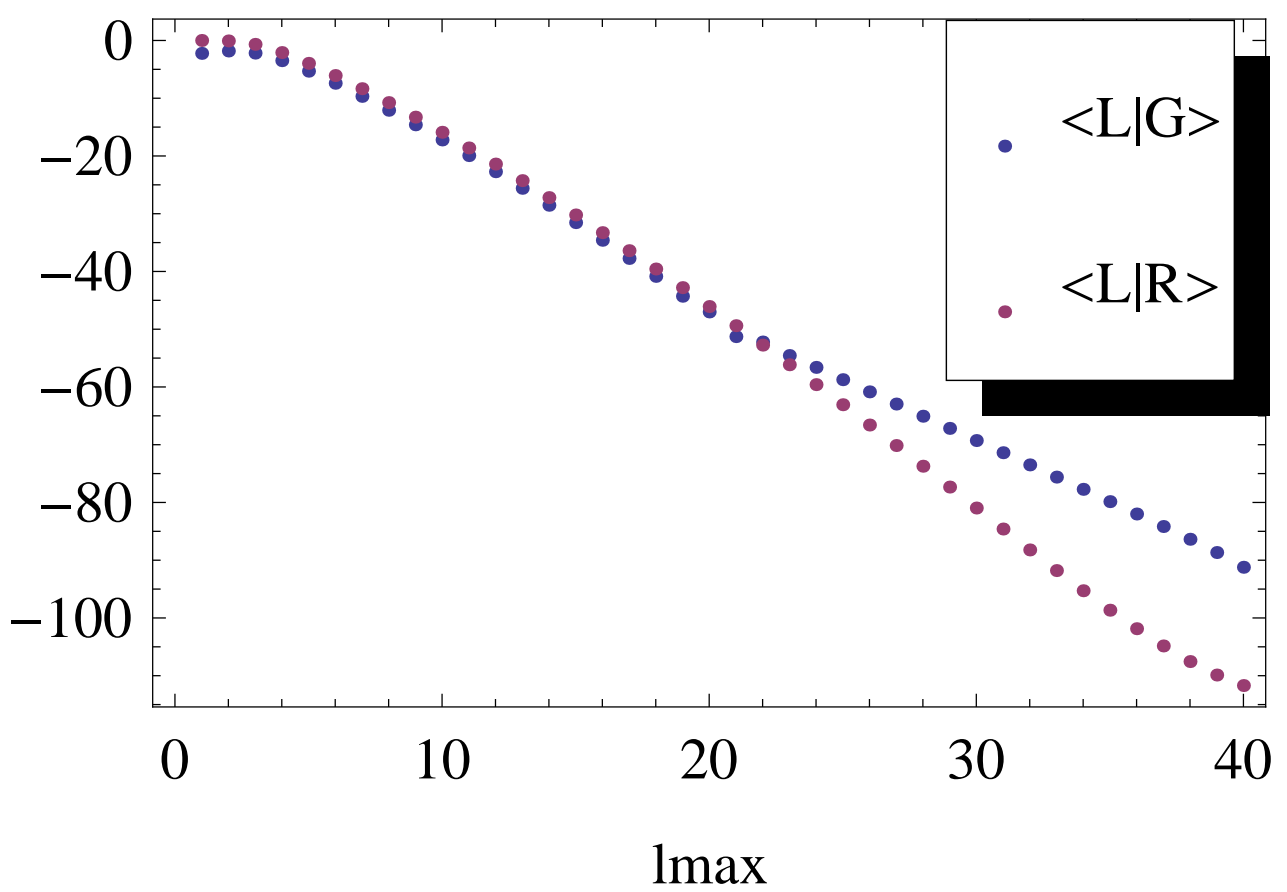

Figure 2.18: Red dots: $\langle L \mid R\rangle$; Blue dots: $\langle L \mid R\rangle$.

\subsubsection{Two Lattice Matching}

In this section, we will discuss how to construct the renormalization group (RG) flow in the complex $\beta$ plane by using the two lattice matching method.

Now, let me briefly introduce the basic idea of the two lattice matching method. For simplicity, we assume there is only one relevant direction for the system studied. We start the RG transformation at one coupling $\beta_{1}$ near the phase transition, after several transformations, the system will go along the relevant direction and follow the flow thereafter. We can try to search for another coupling $\beta_{2}$, where we only need to do one less time the transformation to reach the relevant direction and match the same point as the previous case in the parameter space. Suppose the scaling factor for the transformation is $b$, lattice spacing a $\rightarrow$ ba, the 


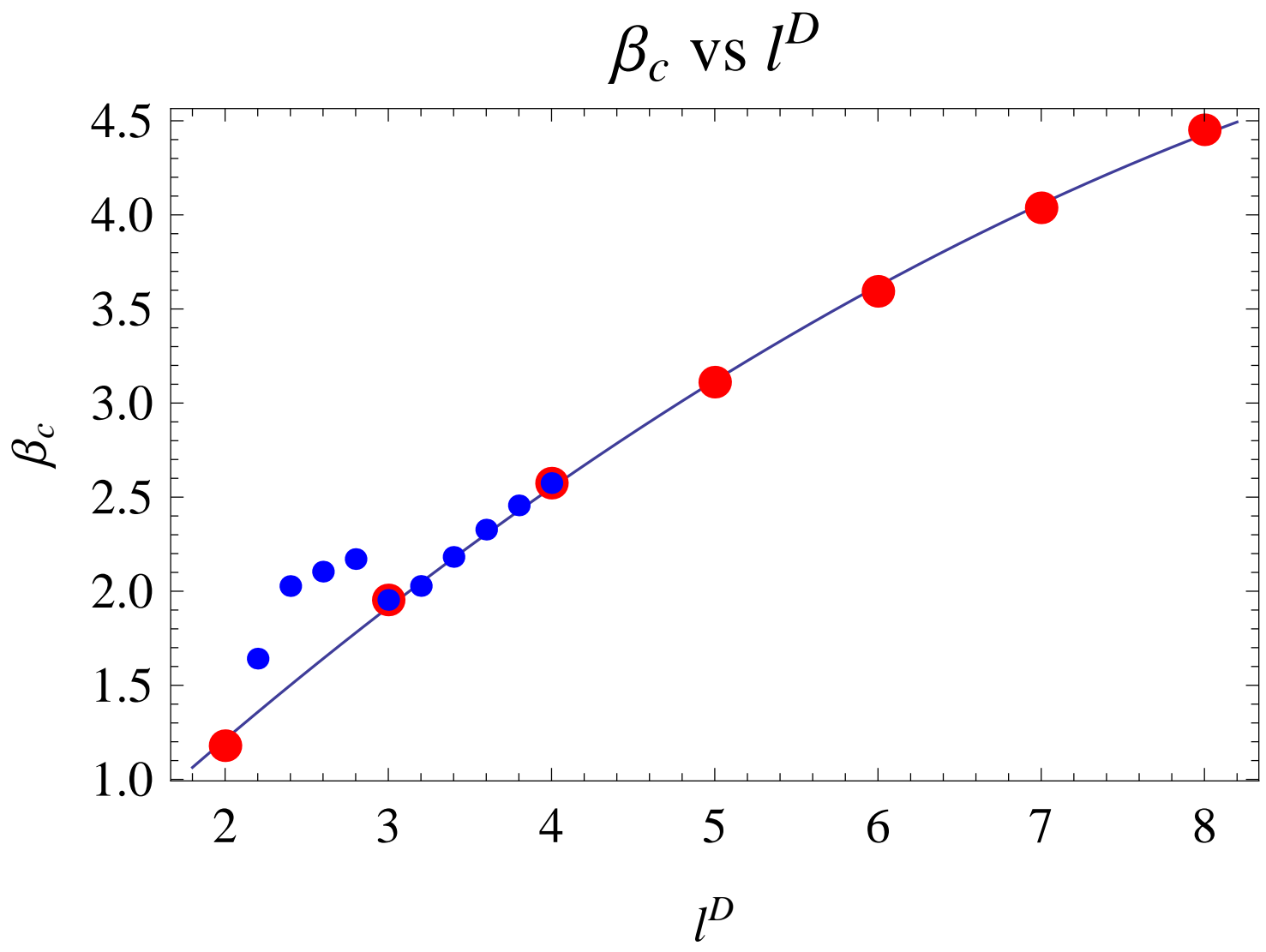

Figure 2.19: The critical temperature $\beta_{c}$ changes with $b^{D}$. 


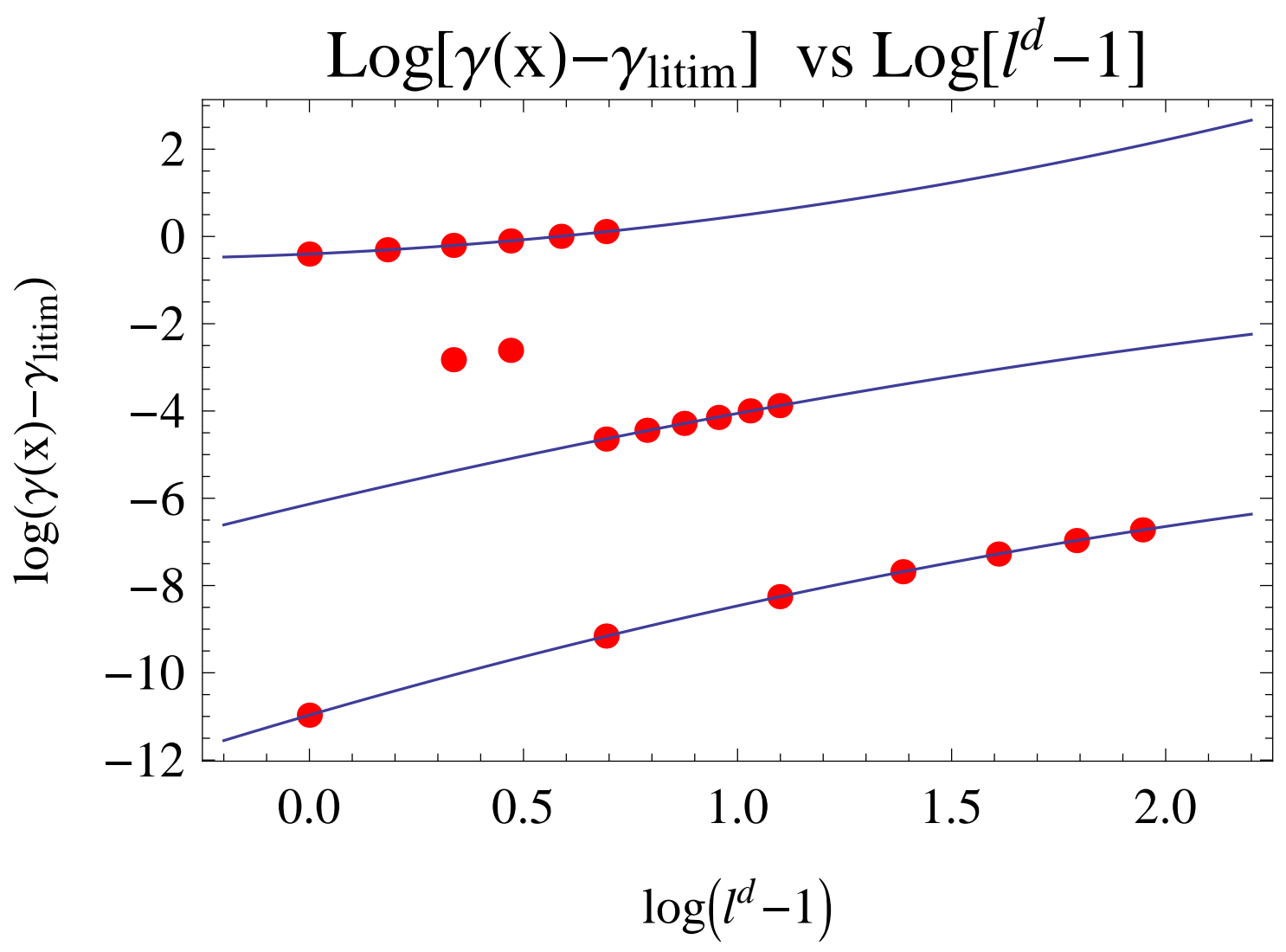

Figure 2.20: The critical exponent $\gamma$ changes with $b^{D}$. 
two $\beta$ 's have the following relationship

$$
\beta_{2}-\beta_{c}=b^{\frac{1}{\nu}}\left(\beta_{1}-\beta_{c}\right)
$$

Usually, one will choose the scaling variable $b=2$. In the hierarchical model described below, $b$ can be non-integer, i.e., $2^{\frac{1}{3}}$. In practice, we will choose two systems with different volume, where the linear size of the two systems differ by a factor of $b$. We want these two systems describe the same long distance physics at $\beta_{1}$ and $\beta_{2}$. For example, we can keep the correlation length $\xi$ in physical units to be constant. We start with one system with volume $L^{D}$, lattice spacing $a$, and coupling $\beta_{1}$. After $n$ iteration, the lattice spacing changes from $a$ to $b^{n} a$. The correlation length in lattice units changes from $\frac{\xi}{a}$ to $\frac{\xi}{b^{n} a}$. $\xi$ is the physical length divided by a, $\xi=\frac{1}{m a}$ where $\mathrm{m}$ is a dimensionful physical mass. We can simulate another system with volume $\frac{L}{b}^{D}$, lattice spacing $b a$, and coupling $\beta_{2}$. This time we iterate $n-1$ times. The lattice spacing changes from $b a$ to $b^{n-1} b a=b^{n} a$. The correlation length in the lattice unit changes from $\frac{\xi}{b a}$ to $\frac{\xi}{b^{n} a}$. They both end up with the same correlation length if we measure them in the lattice unit. We further assume they have the same action in the blocked lattice. Usually the blocked action will involve a large number of couplings and it is impractical to calculate it. However, we do not need to calculate it directly. We use the fact that when the action of the system is the same, all the observables should also be the same. We only need to measure a set of different observables. For details, please see [141].

The observable we use to match is the spin-spin correlation, which is a little different from that of Ref. $[23,25]$. We will see it in the following that the observable $R\left(\beta, 2^{N}\right)$ defined in 2.79 does not depend on the lattice rescaling.

Suppose we have $2^{N}$ sites in the whole volume. We can split the system into two blocks $B_{1}$ and $B_{2}$. Each of them has $2^{N-1}$ sites. Figure $(2.21)$ is an illustration 
for $D=1$ case. The observable we considered is

$$
R\left(\beta, \mathcal{V} / a^{D}\right) \equiv \frac{\left\langle\left(\sum_{x \in B_{1}} \boldsymbol{\phi}_{x}\right)\left(\sum_{y \in B_{2}} \boldsymbol{\phi}_{y}\right)\right\rangle_{\beta}}{\left.\left\langle\left(\sum_{x \in B_{1}} \boldsymbol{\phi}_{x}\right)\left(\sum_{y \in B_{1}} \boldsymbol{\phi}_{y}\right)\right)\right\rangle_{\beta}}
$$

where $\mathcal{V}$ is the physical volume of the system and $a$ is the lattice spacing in the physical unit(i.e., in the unit of meter, centimeter, etc.). The observable $R$ has the following advantages: 1), it is relatively easy to calculate for the system considered; 2 ), it does not require to calculate the partition function; 3), we do not need to consider the field rescaling associated with the full RG transformation. Both 2) and 3) are due to the rational form of the observable $R$. The observable can be calculated both in $\phi$ space and $k$ space. In $k$ space, the model can be very easily extended to the $O(N)$ model with arbitrary $N$ and arbitrary dimension $D$. This enables us to extend the technique developed here over a wide range of interesting questions.

In the following, we will give the details on how to calculate $R$. From the relationship between the measures in the successive hierarchy (see Equation 2.13), we can immediately get the following formula

$$
\begin{aligned}
& \left\langle\left(\sum_{x \in B_{1}} \phi_{x}\right)\left(\sum_{y \in B_{2}} \phi_{y}\right)\right\rangle_{\beta} \\
= & \frac{\int d \phi_{1} d \phi_{2} e^{\frac{\beta}{2}\left(\frac{c}{4}\right)^{N}\left(\phi_{1}+\phi_{2}\right)^{2}} \phi_{1} \phi_{2} W_{N-1}\left(\phi_{1}\right) W_{N-1}\left(\phi_{2}\right)}{Z_{N}},
\end{aligned}
$$

and

$$
\begin{aligned}
& \left\langle\left(\sum_{x \in B_{1}} \boldsymbol{\phi}_{x}\right)\left(\sum_{y \in B_{1}} \boldsymbol{\phi}_{y}\right)\right\rangle_{\beta} \\
= & \frac{\int d \phi_{1} d \phi_{2} e^{\frac{\beta}{2}\left(\frac{c}{4}\right)^{N}\left(\phi_{1}+\phi_{2}\right)^{2}} \phi_{1}^{2} W_{N-1}\left(\phi_{1}\right) W_{N-1}\left(\phi_{2}\right)}{Z_{N}} .
\end{aligned}
$$

As mentioned above, we do not need to calculation the partition function $Z_{N}$ in the denominator to calculate the ratio in Eq. 2.79. Only the numerators matter. The local measure $W_{N-1}$ can be constructed easily from $W_{0}$ and hence the 


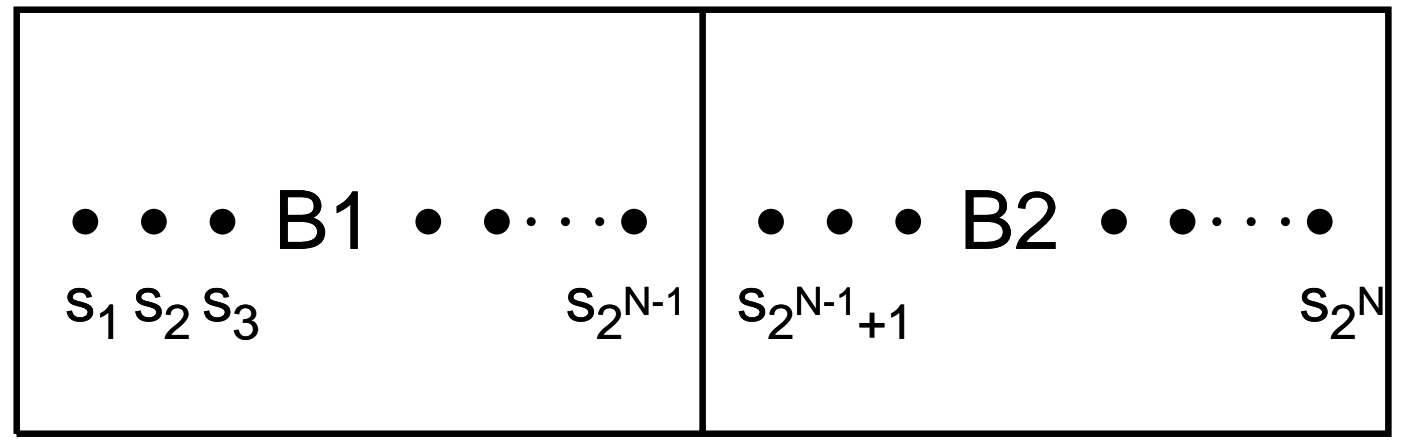

Figure 2.21: An example of blocking for the one dimensional hierarchical model. 
observable $R$.

In the $k$ space, the calculation is a little tricky. We notice that the calculation of $\left\langle\left(\sum_{x \in B_{1}} \phi_{x}\right)\left(\sum_{y \in B_{2}} \boldsymbol{\phi}_{y}\right)\right\rangle_{\beta}$ is similar to that of the partition function except that we need to replace $W(\phi)$ by $\phi W(\phi)$ or $\phi^{2} W(\phi)$.

$$
\begin{aligned}
\phi W(\phi) & =\phi \int \frac{d k}{2 \pi} e^{i k \phi} R(k) \\
& =\int \frac{d k}{2 \pi}\left(-i \frac{\partial}{\partial k} e^{i k \phi}\right) R(k) \\
& =\int \frac{d k}{2 \pi} e^{i k \phi}\left(i \frac{\partial}{\partial k} R(k)\right)+\int \frac{d k}{2 \pi}\left(-i \frac{\partial}{\partial k}\left(e^{i k \phi} R(k)\right)\right)
\end{aligned}
$$

where the last term can be set to 0 if $R(k)$ decays fast enough at $\pm \infty$. We can perform the same trick to the $\phi^{2} W(\phi)$ term. After several lines of simple algebra, we will get

$$
R\left(\beta, 2^{N}\right)=\frac{e^{-\frac{\beta}{2}\left(\frac{c}{4}\right)^{N} \nabla_{k}^{2}}\left(-\left(R_{N-1}^{\prime}(k)\right)^{2}\right)}{e^{-\frac{\beta}{2}\left(\frac{c}{4}\right)^{N} \nabla_{k}^{2}}\left(-R_{N-1}^{\prime \prime}(k) R_{N-1}(k)\right)}
$$

In practical calculations, we expand the operator $e^{-\frac{\beta}{2}\left(\frac{c}{4}\right)^{N} \nabla_{k}^{2}}$ up to certain orders and approximate the local measure by polynomials. We need to increase the order of the polynomial until the result of the observable stabilizes.

For the $O(N)$ model, we only need to change the initial measure $W_{0}(\phi)$ from $\delta\left(\phi^{2}-1\right)$ to $\delta\left(\phi^{2}-1\right)$. We have already mentioned that changing the dimensionality $D$ is equivalent to changing the strength parameter $c: c / 4=b^{-2-D}$.

Having figured out how to calculate the observable, we use the two lattice matching method to construct the RG flows. The flows are constructed by the following procedure. We first choose an initial $\beta_{0}$ and then calculate the observable at $\beta_{0}$ with the larger volume, say $2^{5}$. We then use the Newton's method or the "logarithmic residue" method to find the corresponding $\beta_{1}$ which gives the same observable at smaller volume, say $2^{4}$. We can further use $\beta_{1}$ to the larger volume and try to match another $\beta_{2}$ to the smaller volume $\cdots$. By doing this, we can get a sequence of $\beta$ s starting from $\beta_{0}$ and ending to $\beta_{n}$. By choosing different initial 
starting points, we can get different flow lines(connecting $\beta_{i}$ and $\beta_{i+1}$ with arrows). Figure (2.22) shows several flow lines starting from $\operatorname{Re} \beta=5$ and different $\operatorname{Im} \beta$. We can see clearly that all the flows stay in the boundary formed by the Fisher zeros and they also all flow to the trivial strong coupling fixed point.

The above matching procedure can be written as

$$
R\left(\beta, 2^{5}\right)=R\left(\beta^{\prime}, 2^{4}\right)
$$

We should notice that there are usually more than one $\beta^{\prime}$ satisfying the matching condition 2.86 for one single $\beta$. This can be easily seen from the case we will discuss in the following.

\subsubsection{1 $D=2$}

For $D=2$ and $b^{D}=2$, we have $c=1$. In this case, all the $\lambda_{k}$ in equation (2.39) are non-negative rational numbers. In general, the least common multiple of the denominator of $\lambda_{k}$ is $V^{2} / 2$, where $V$ is the volume of the system. The matching condition 2.86 also has the similar property. This nice property allows us to write the matching condition as a polynomial equation by changing the variable $\beta^{\prime}$ to $V^{2} / 2 \log [n]$. Searching for the matching point $\beta^{\prime}$ reduces to solving for the roots of the polynomial equation. The number of the solutions depends on the order of the polynomial. That explains why there are more than one $\beta^{\prime}$ for a fixed $\beta$. In order to get unambiguous flows, we need to develop some strategy to pick the "right" matching $\beta^{\prime}$. A natural way is to compare the distance between $\beta$ and the $\beta^{\prime}$ and choose the closest one to the $\beta$ as the matching point. We found out that most of the time it is easy to determine the closest $\beta^{\prime}$ because the other matching candidates are all very far away from the initial $\beta$. In order to quantify the ambiguity of choosing the closest matching $\beta^{\prime}$, we define the following factor

$$
f(\beta)=\log \left|\beta-\beta_{2}^{\prime}\right|-\log \left|\beta-\beta_{1}^{\prime}\right|,
$$




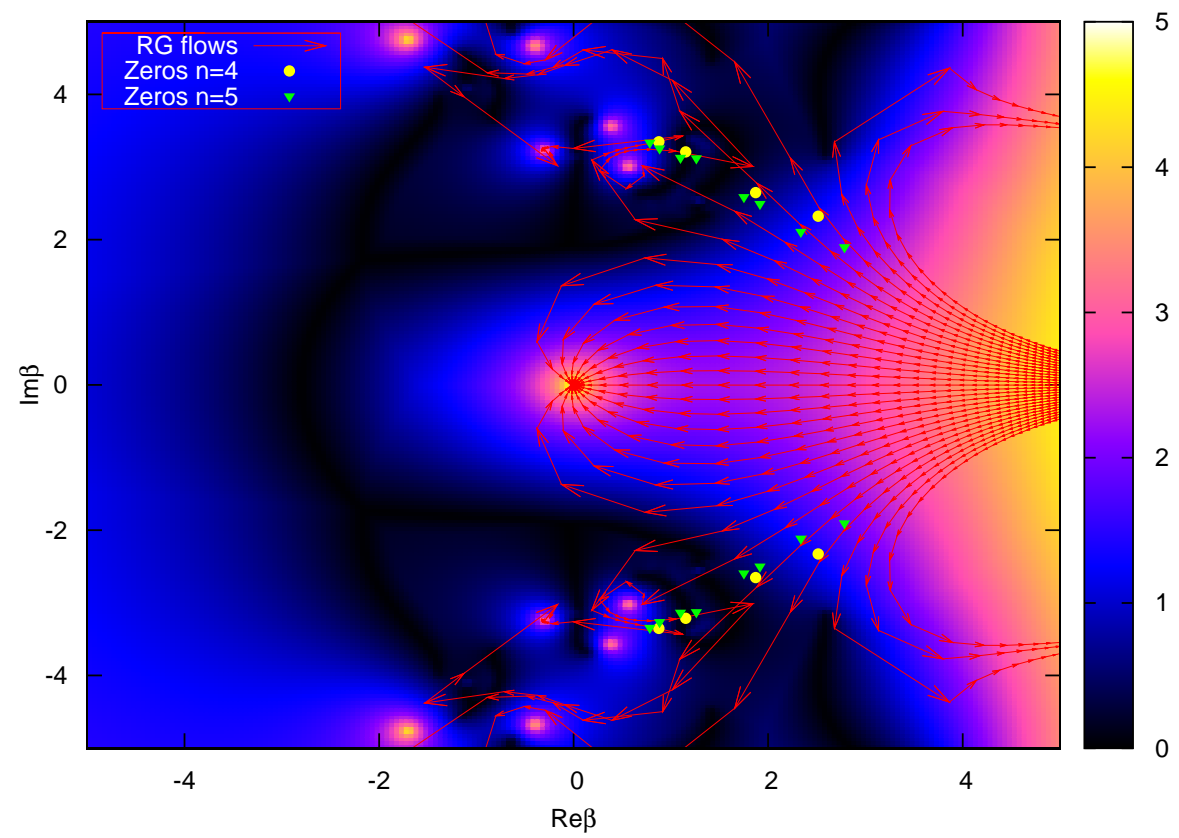

Figure 2.22: RG flows obtained by the two lattice matching methods for hierarchical Model.

where $\beta_{1}^{\prime}$ and $\beta_{2}^{\prime}$ are the closest and next closest $\beta^{\prime}$ to $\beta$. If $f(\beta)$ is very close to zero, $\beta_{1}^{\prime}$ and $\beta_{2}^{\prime}$ are very close to each other. That means that it is more ambiguous to choose the closest $\beta_{1}^{\prime}$ as the matching point. On the other hand, it is less ambiguous if $f(\beta)$ is large. In figure 2.22, we make a contour plot of $f(\beta)$. The darker the color, the closer the $\beta_{1}^{\prime}$ to $\beta_{2}^{\prime}$, in another word, the more ambiguous to pick $\beta^{\prime}$. Notice that the flows near the Fisher zeros are more ambiguous and unpredictable.

\subsubsection{2 $\quad D=3$}

As stated in the previous section, there is a phase transition for the $D=3$ case and the critical $\beta$ is around 1.179 in the infinite volume limit. In Figure 2.23, we can clearly see that the flow goes from the nontrivial fixed point to the trivial fixed points 0 and $\infty$. 


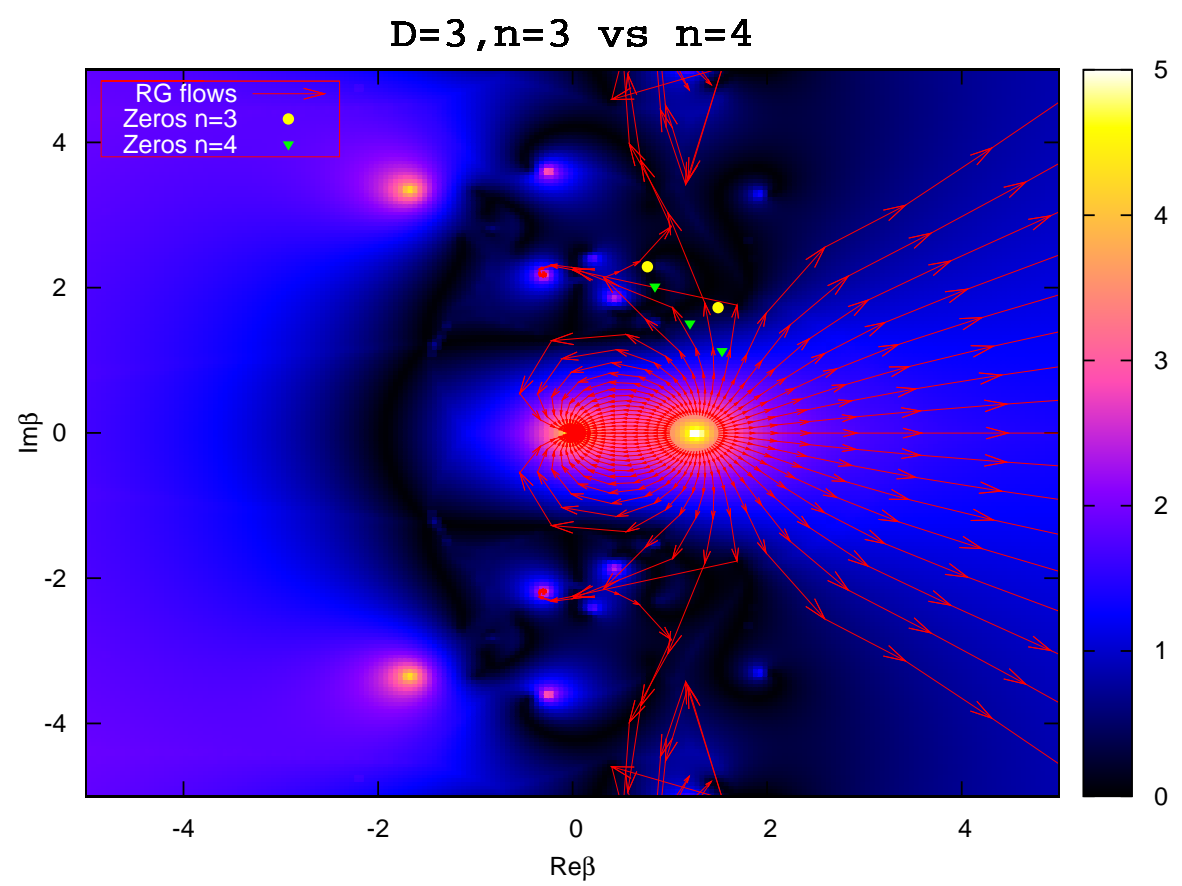

Figure 2.23: Unambiguous RG flows for the hierarchical model in the complex $\beta$ plane obtained by the two lattice method.

One of the motivations of doing the two lattice matching for the hierarchical model was to construct the RG flow in the complex parameter plane and find connections between the flow and the Fisher zeros. One can further construct the $\beta$ function, which is similar to the Callan-Symanzik $\beta$ function used in the field theory language. We will discuss more about that in the lattice gauge theories chapters later.

The matching condition can be chosen as

$$
R\left(\beta, L^{D}\right)=R\left(\beta^{\prime},(L / b)^{D}\right)
$$

We can then start from the bare value $\beta_{0}$ and get the new coupling from Eq. 2.86

$$
\begin{aligned}
& \beta=\beta_{0} \Longrightarrow \beta_{1}^{\prime}, \\
& \beta=\beta_{1}^{\prime} \Longrightarrow \beta_{1}^{\prime \prime},
\end{aligned}
$$


The discrete $\beta$ function is then defined as

$$
\Delta \beta\left(\beta, L^{D} \rightarrow(L / b)^{D}\right)=\beta-\beta^{\prime}
$$

In the following, we will consider the further generalized hierarchical model with $2^{n_{\max }}$ lattice sites

$$
H=-\frac{1}{2} \sum_{n=1}^{n_{\max }}\left(\frac{c}{4}\right)^{n} f(n) \sum_{B^{(n)}}\left(\sum_{x \in B^{(n)}} \phi_{x}\right)^{2} .
$$

All the other parameters are the same as the conventional one except that there is an extra $f(n)$ factor, which further controls the interaction strength. The generalized model has many nice properties and is an ideal laboratory to test various ideas before one applies them to the more complicated full QCD case. Here is a list of properties we used $[82,84,142]$

- For $D>2$ and $f(m)=1$, the model has a second order phase transition, which is similar to $\mathrm{D}=3$ regular Ising model.

- For $D \leq 2$ and $f(m)=1$, the model has no phase transition at finite temperature, which is different from $\mathrm{D}=2$ regular Ising model.

- For $D=2$ and $f(m)=\log (m)$, the model has Thouless effect, which is equivalent to Anderson model.

In the following, we will consider $\mathrm{D}=3,2, f(m)=1$ and various dimensions for $f(m)=\log (m)$. One of the reasons to investigate systems with different parameters is to get better understanding on how infrared fixed point (IRFP) emerges, which plays a key role in understanding the techni-color related theories in the high energy physics field. Fixed points correspond to zeros of the $\beta$ function. It has been pointed out that zeros of the $\beta$ function can disappear in three ways as one or several 


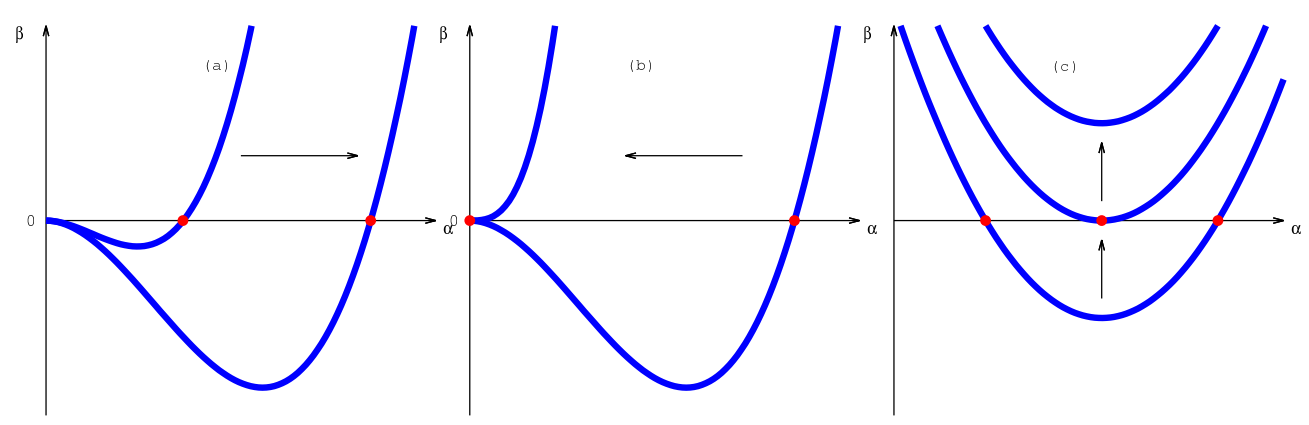

Figure 2.24: Three mechanisms for the loss of the fixed point(s) described in [2].

parameters change [2]. They are schematically described in Fig. 2.24. The first two ways are interchangeable depending on what parameters are used $(\alpha \leftrightarrow 1 / \alpha)$. For the third way, the fixed points that disappeared can be recovered in the complex parameter plane [143]. This third way can be easily seen for the hierarchical model by tuning the dimensionality $D$. Figure 2.27 shows discrete $\beta$ functions for $f(n)=$ $\log (n+1), D=1.9,1.994$, and 2 . The corresponding complex RG flows are shown in Fig. 2.28, 2.29, 2.30. Figure 2.31 shows that Fisher zeros appear to accumulate along lines that separate the flows ending on different fixed points. 


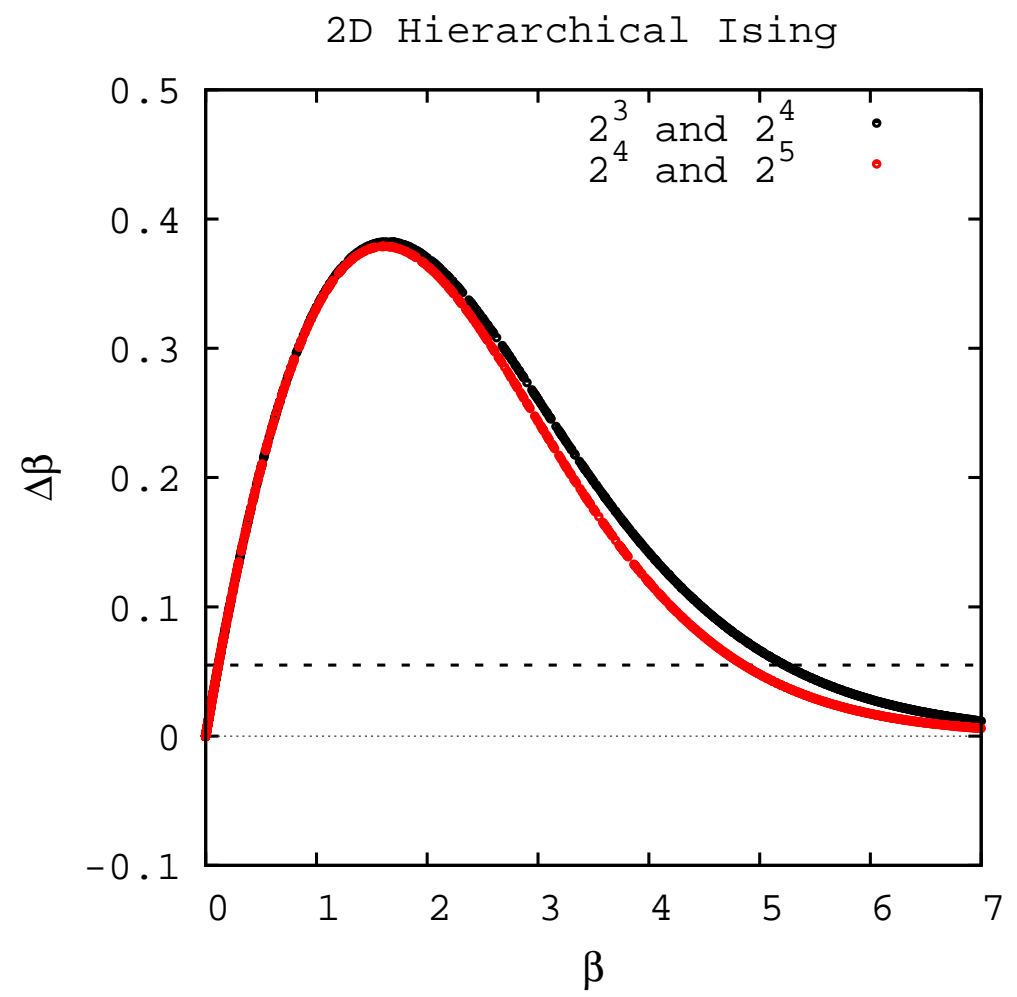

Figure 2.25: Discrete $\beta$ function for the hierarchical model with $D=2$ and $f(m)=$ 1. 


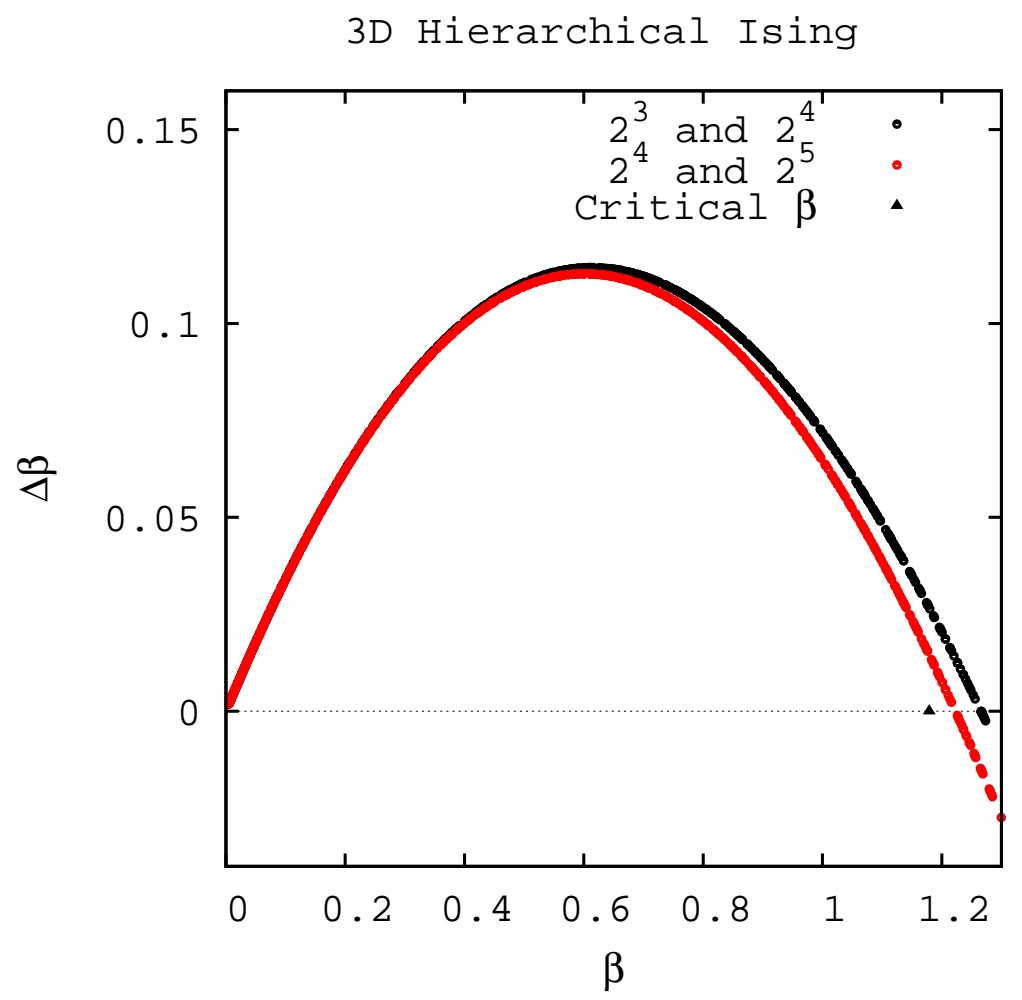

Figure 2.26: Discrete $\beta$ function for the hierarchical model with $D=3$ and $f(m)=$ 1. 


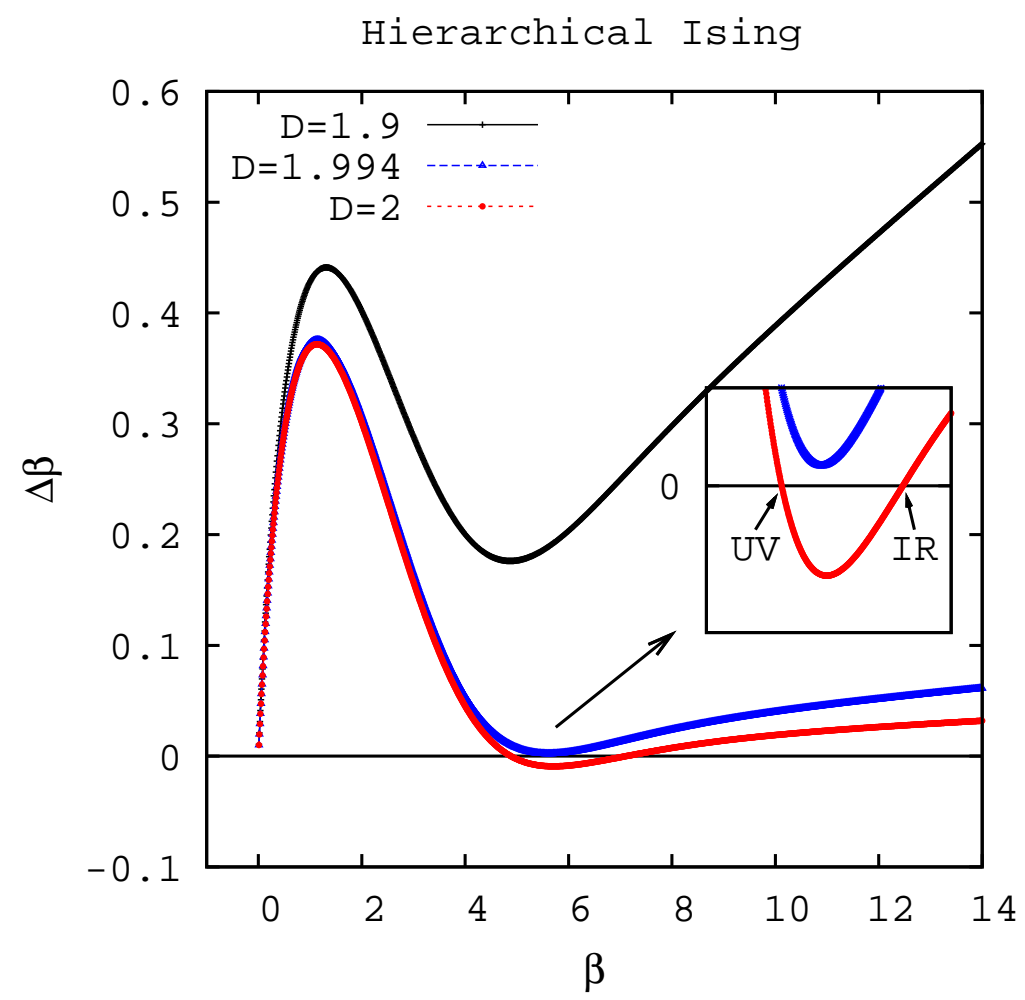

Figure 2.27: Discrete $\beta$ function for the hierarchical model with $D=1.9,1.994$, and 2 and $f(m)=\log (m)$. 


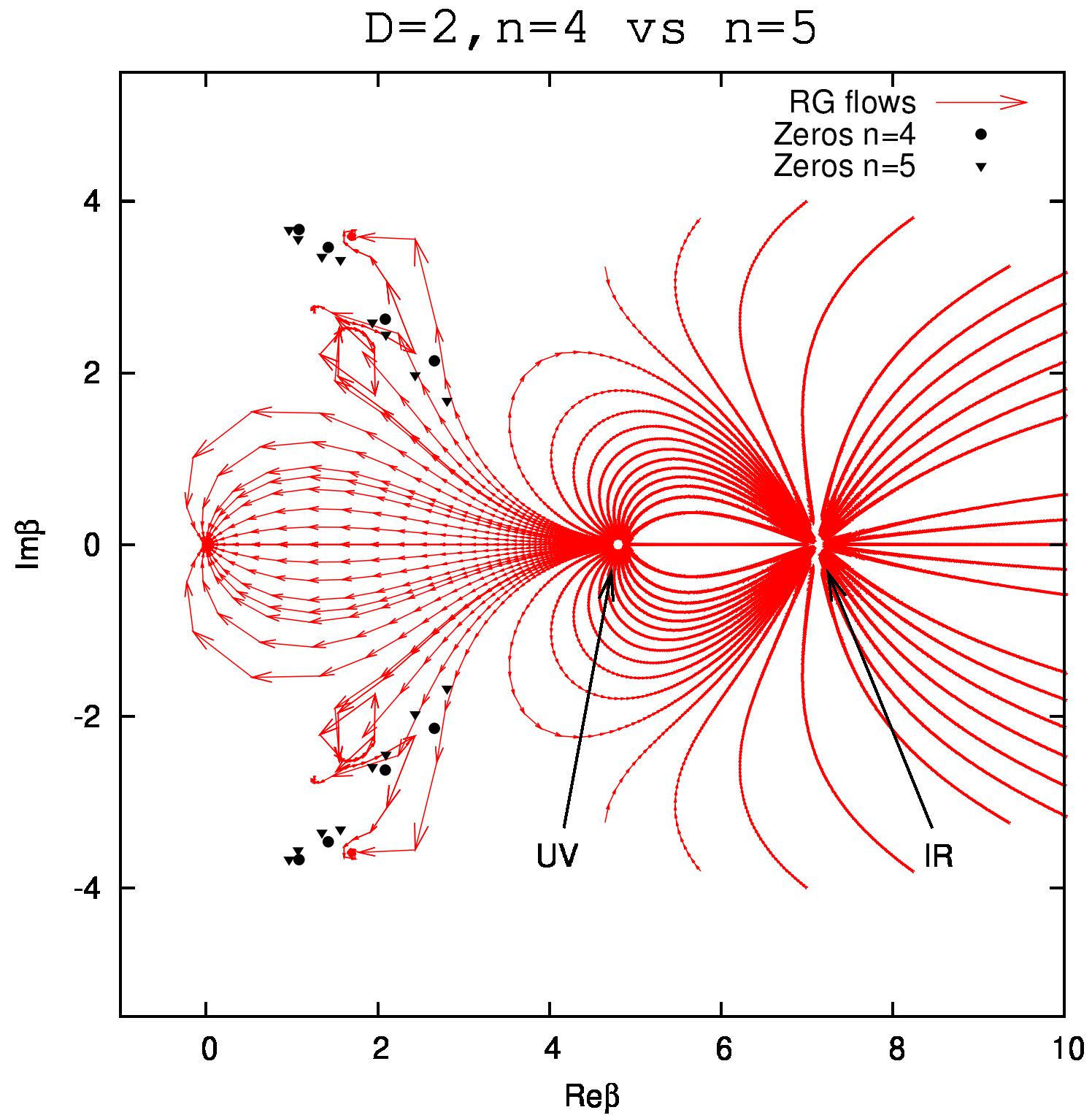

Figure 2.28: Complex RG flows for $D=2$. 


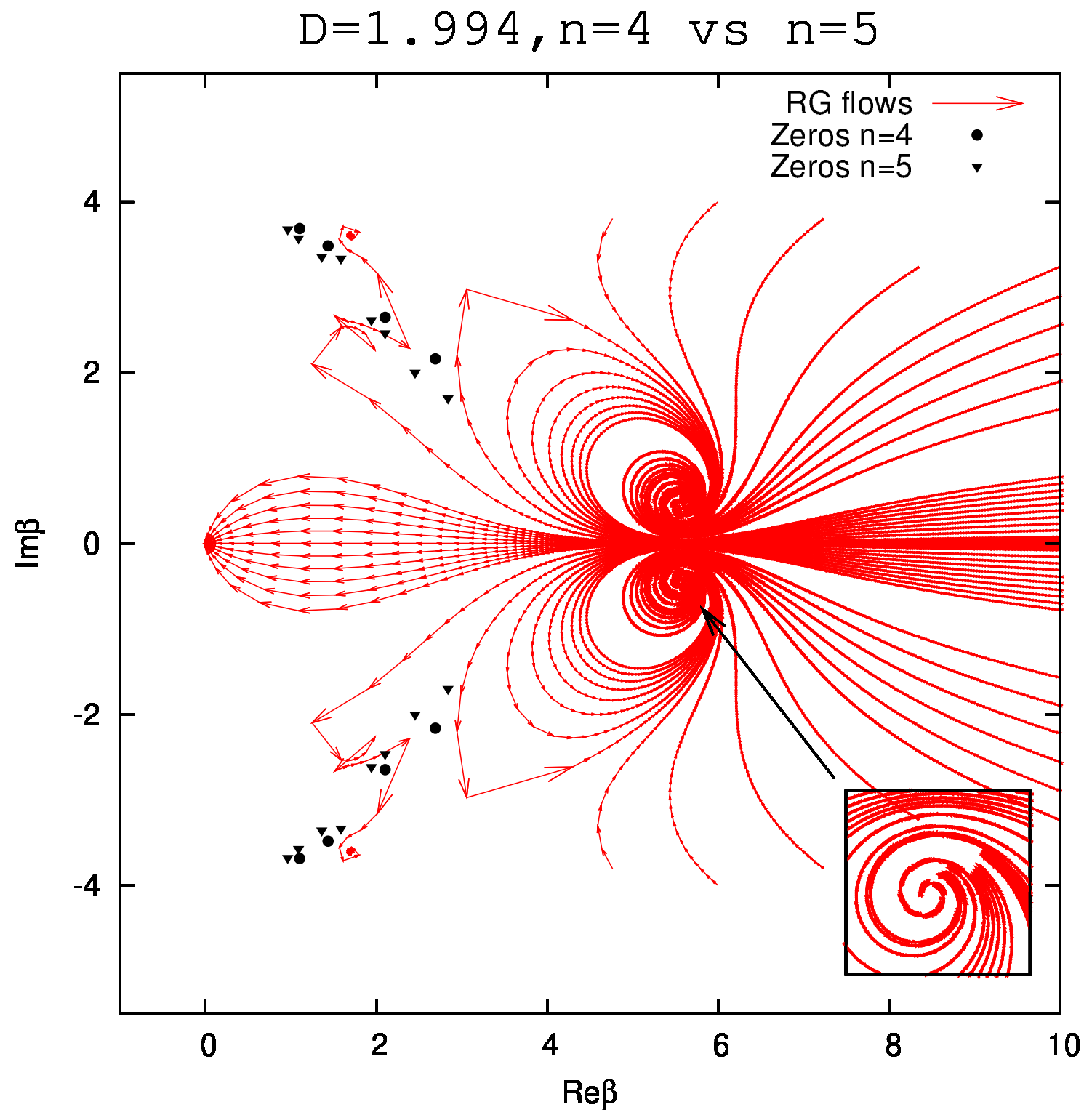

Figure 2.29: Complex RG flows for $D=1.994$. 


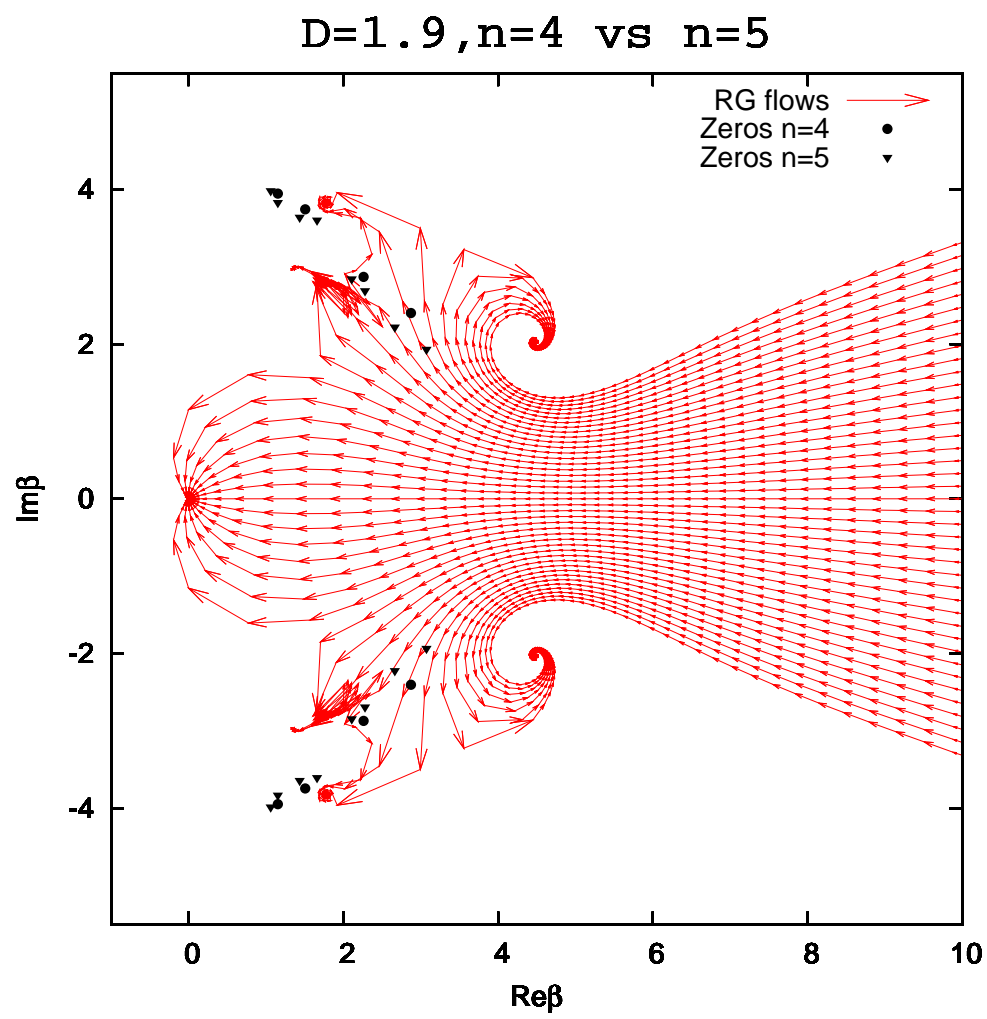

Figure 2.30: Complex RG flows for $D=1.9$. 


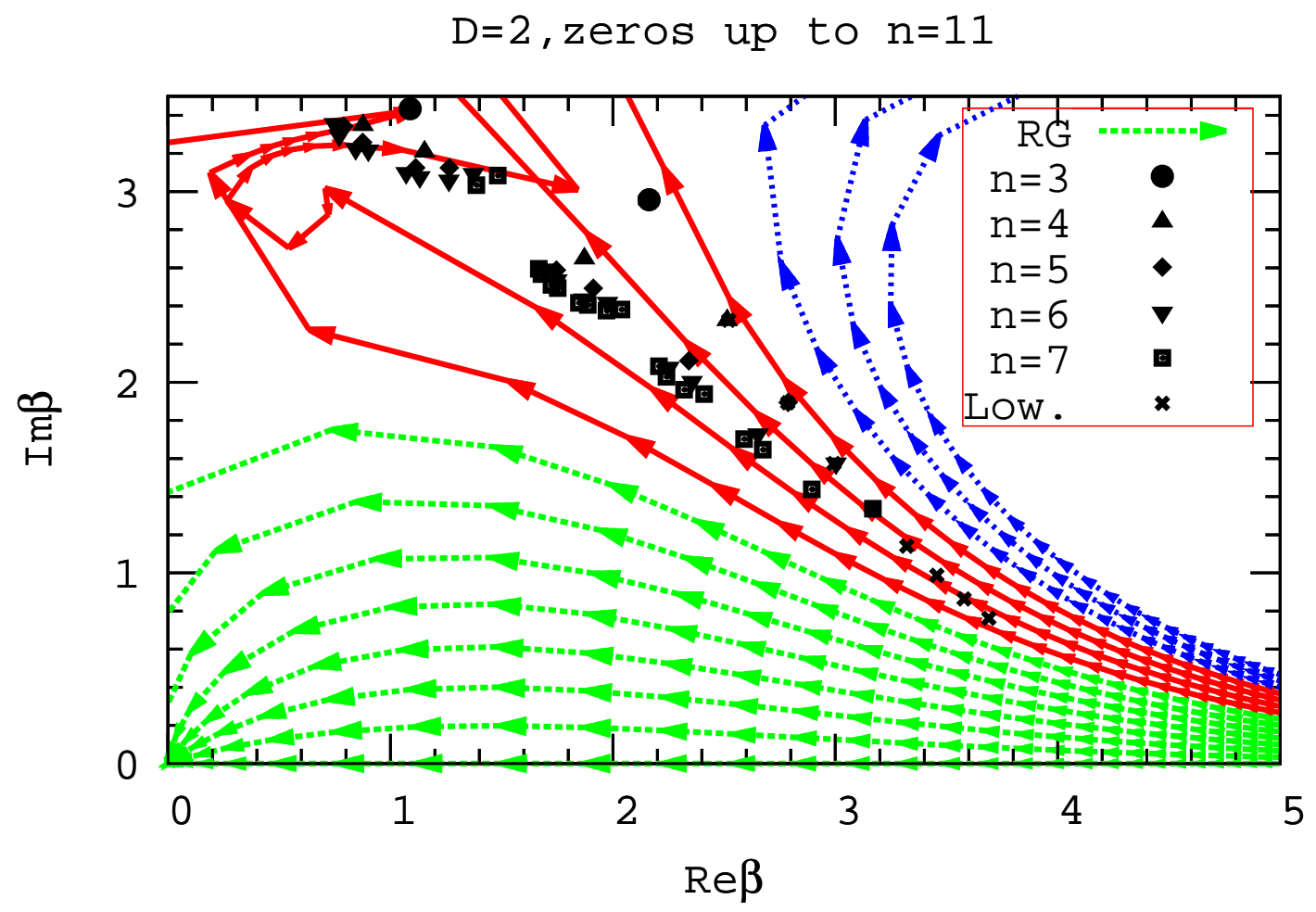

Figure 2.31: The density of the zeros is proportional to the $\ln V$. 


\section{CHAPTER 3 EXACT RENORMALIZATION GROUP}

\subsection{Introduction}

In section 2.2, we see that the critical exponent $\gamma$ of the hierarchical model depends on the blocking scheme. This is a very unpleasant result. In general, the critical exponent should be independent of the blocking. We plan to find methods to improve the hierarchical model. We will start with the hierarchical model and find a scheme independent model which has the same nice properties as the hierarchical model and at the same time can approximate the nearest neighbor model in D dimensions.

On the other hand, Daniel Litim [3] pointed out the critical exponents $\nu$ and $\omega$ calculated by the exact renormalization group (ERGE) method under local potential approximation (LPA) are highly correlated. He showed that the critical exponents of the hierarchical model with $b^{D}=2$ also fell into the same curve (Fig. 3.1). We

further show that all the other results with integer $b^{D}$ also nicely fit into this curve. We are interested in knowing why this is the case. In the following section of this chapter, we will discuss the technique of the ERGE and its connection with the RG discussed in the previous chapter.

The ERGE was first introduced by Wilson [144], and then Wegner and Houghton [145] in the 1970s. Later on, it was reformulated by Polchinski [131] in 1984. ERGE is a continuous realization of the Wilson renormalization group. "Exact" comes from the fact that there is no approximation or expansion involved in the renormalization group (RG) transformation of the action. However, due to the complexity of the formulation, approximation or truncation are usually needed in order to perform practical calculations. Therefore, ERGE calculations are usually not "exact" and some people prefer the term "functional renormalization group". 


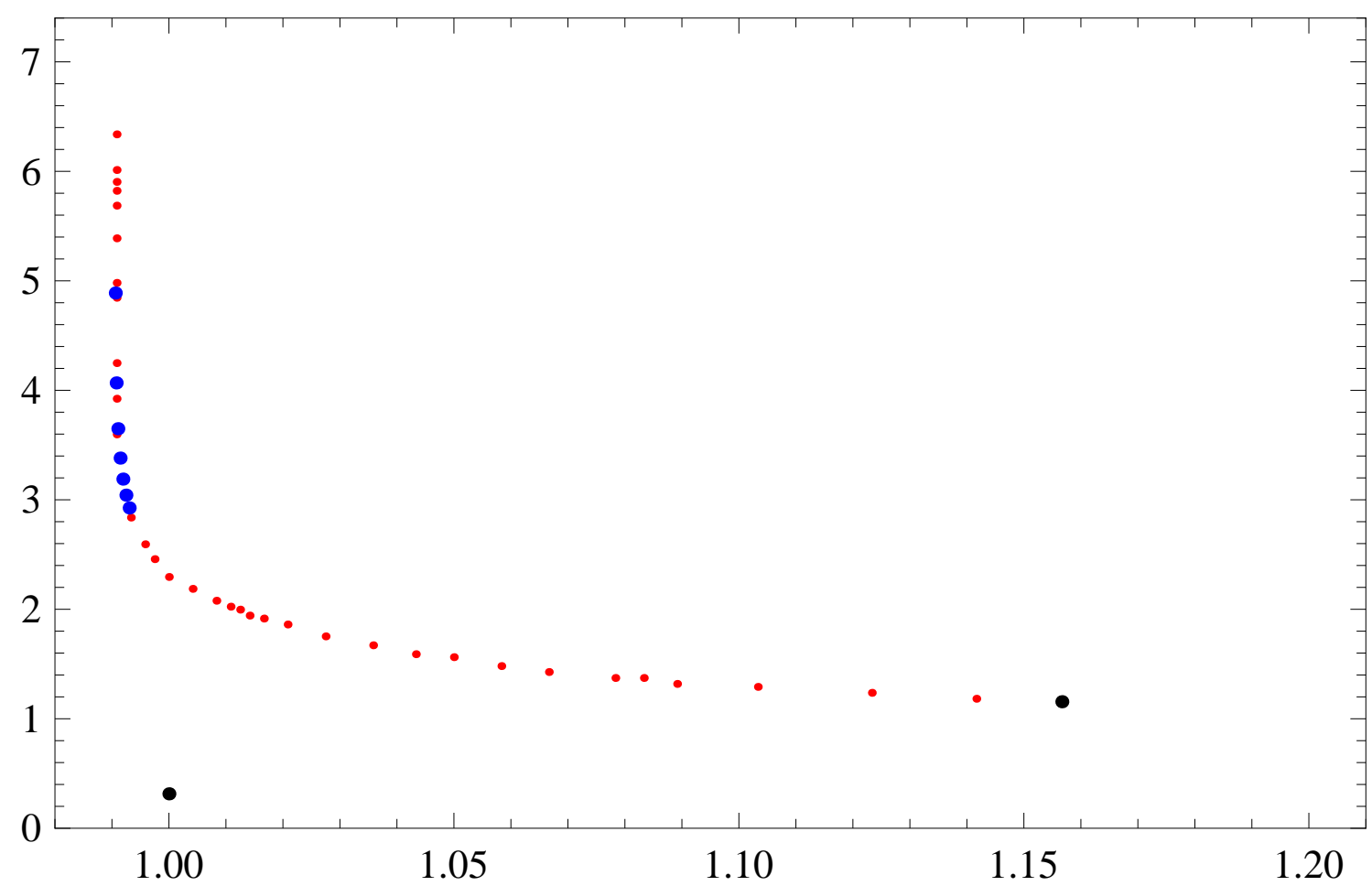

Figure 3.1: The red dots data were obtained from Fig. 4 in Ref. [3].

ERGE has been used in many sub-field of the physics. References can be found in the review articles $[129,146,147,148]$ and textbooks $[149,78,150,151]$.

In this chapter, we will mainly focus on the Wilson-Polchinski ERGE [144, 131], where the cutoff is smooth, under the local potential approximation (LPA) of the derivative expansion. We will also show the equivalence between the WilsonPolchinski equation and the Litim optimized renormalization group equation $[152$, $153,154,155]$.

\subsection{Overview}

Renormalization group method has been used successfully in quantum field theory and condensed matter fields to calculate the critical exponents and other observables near the critical region. Wilson renormalization group $(\mathrm{RG})$ is based on the idea of integrating out the high momentum degrees of freedom successively and 
therefore interpolating between the low momentum and high momentum physics. There are many different realization of this process and the obvious advantage is that one can do various flexible approximations. Furthermore, optimization technique can be used to increase the reliability of the calculation.

For completeness, let us re-derive the formula we are interested in. The partition function with classical action $S$ and a source term $J$ can be written as

$$
Z_{k}[J]=\int \exp \left(-S[\phi]+\int d^{d} x J(x) \phi(x)\right)
$$

where $d$ is the dimensionality of the system and $\phi$ is the scalar field. Let us then add a cutoff term $\Delta S_{k}[\phi]$, which takes into account of the rescaling of the field $\phi$ and keeping the original partition function invariant. One usually only consider the oneloop structure of the flow equation because higher loops will introduced complicated loop integration overlaps. The one-loop requirement constrains the cutoff term to be at most quadratic. Therefore, we can write

$$
\delta S_{k}[\phi]=\frac{1}{2} \int d^{d} x \phi R \phi .
$$

Here $R$ is the cutoff function depending on the infrared scale $k$. The role of $R$ is to suppress the low momentum mode while keep the high momentum mode intact. It also has to keep the correct initial condition $\left.\Gamma_{k}\right|_{k \rightarrow \Lambda}=S_{\Lambda}$. Therefore, we will need $R$ to satisfy the following condition

$$
\begin{aligned}
\lim _{q^{2} / k^{2} \rightarrow 0} R_{k}\left(q^{2}\right) & >0, \\
\lim _{k^{2} / q^{2} \rightarrow 0} R_{k}\left(q^{2}\right) & =0, \\
\lim _{k \rightarrow \Lambda} R_{k}\left(q^{2}\right) & \rightarrow \infty .
\end{aligned}
$$

The flow equation for the Legendre transformed effective action $\Gamma_{K}[\phi]=$ $\int d^{d} x J(x) \phi(x)-\ln Z_{k}[\phi]-\Delta S_{k}[\phi]$ is $[156,157,158,159]$ 


$$
\partial_{t} \Gamma_{k}[\phi]=\frac{1}{2 \operatorname{Tr}\left(\frac{\partial^{2} \Gamma_{k}[\phi]}{\partial \delta \phi(p) \delta \phi(q)}+R_{k}\right)} \partial_{t} R_{k},
$$

where $t=\ln k$ is the logarithmic scale parameter, $\operatorname{Tr}$ means integrating over momentum and summing over indices.

The flow is linear and infrared and ultraviolet finite. Using the derivative expansion [160], to leading order in other words the local potential approximation (LPA) $[144,161,162]$, the effective action of the $O(N)$ symmetric scalar theory has the following form

$$
\Gamma_{k}=\int d^{d} x\left(V_{k}(\phi)+\frac{1}{2} \partial_{\nu} \phi \partial_{\nu} \phi\right)
$$

where $V_{k}(\phi)$ is the local potential. From Eq. 3.6 and 3.7, we can get the flow equation for $V_{k}$ field. If we rewrite the flow equation in terms of the dimensionless variables $u(\rho)=V_{k} / k^{d}$ and $\rho=1 / 2 \phi^{2} k^{2-d}$ and taking the proper trace [163], we obtain

$$
\partial_{t} u(\rho)=-d u(\rho)+(d-2-\eta) \rho u^{\prime}+\frac{K_{d}}{2}(N-1) l\left(u^{\prime}\right)+\frac{K_{d}}{2} l\left(u^{\prime}+2 \rho u^{\prime \prime}\right),
$$

where $K_{d}=\frac{2 \pi^{d / 2}}{(2 \pi)^{d} \Gamma(d / 2)}$ is the surface of the $d$ dimensional unit sphere divided by $(2 \pi)^{d}$. The function $l(\omega)$ is defined as

$$
l(\omega)=\frac{1}{2} \int_{0}^{\infty} d y y^{d / 2} \frac{\partial_{t} r(y)}{y(1+r)+\omega},
$$

where $y \equiv q^{2} / k^{2}$ and $\partial_{t} r(y)=-2 y r^{\prime}(y)$. Here $r(y)$ is the dimensionless regulator function

$$
R\left(q^{2}\right)=q^{2} r\left(\frac{q^{2}}{k^{2}}\right)
$$

As stated before, one can choose different cutoff functions $r(y)$ as long as they satisfy the proper conditions. Different cutoff functions correspond to different coarse-graining process. Unfortunately, solutions to the truncated flow equation 3.6 depend on the choice of the cutoff function. This is similar to the perturbative QCD [164] case, where at finite order in perturbation theory different regularization 
schemes gives different physical observables. It was found that there is an optimized cutoff function which leads to faster convergence and stable flow [165]. The function has the following form

$$
r(y)_{\mathrm{opt}}=\left(\frac{1}{y}-1\right) \theta(1-y) .
$$

In the following sections, we will mainly focus on the optimized cutoff function 3.11 and its variants.

\subsection{Numerical Methods To Find The Fixed Points}

Let us start from the flow equation 3.8 and 3.9 For $d=3, \eta=0$ and $N=1$, we obtain

$$
-3 u+\rho u^{\prime}+\int_{0}^{\infty} d y \frac{-y^{\frac{5}{2}} r^{\prime}(y)}{y(1+r)+u^{\prime}+2 \rho u^{\prime \prime}}=\partial_{t} u .
$$

Note that in Eq. (2) of [166] we believe that it should be $y^{5 / 2}$ instead of $y^{3 / 2}$. If we choose the cutoff function to be $r(y)=b\left(\frac{1}{y}-1\right) \theta(1-y)$, where $\theta(x)$ is Heaviside step function, after proper rescaling of the field we obtain

$$
-3 u+\rho u^{\prime}+\frac{1}{4 \pi^{2}} \frac{2 b\left(\sqrt{\frac{b+w}{b-1}} \operatorname{ArcTanh} \sqrt{\frac{b-1}{b+w}}-1\right)}{b-1}=\partial_{t} u \text { for } b>1,
$$

and

$$
-3 u+\rho u^{\prime}+\frac{1}{1+u^{\prime}+2 \rho u^{\prime \prime}}=\partial_{t} u \quad \text { for } \quad b=1,
$$

with $w=u^{\prime}+2 \rho u^{\prime \prime}$.

Let us focus on the $b=1$ case first and the $b>1$ case can be treated with the same technique after taking the series expansion.

The fixed point equation corresponds to $\partial_{t} u^{\prime}=0$. Since the integration constant does not affect the fixed point properties, we can equivalently write

$$
-3 u+\rho u^{\prime}+\frac{1}{1+u^{\prime}+2 \rho u^{\prime \prime}}=0
$$

or equivalently

$$
\left(-3 u+\rho u^{\prime}\right) *\left(1+u^{\prime}+2 \rho u^{\prime \prime}\right)+1=0 .
$$


Equation (3.14) has two fixed points, one is the trivial Gaussian fixed point $u_{\star}=$ Constant and another one is the interesting non-trivial Wilson-Fisher fixed point. In this section, we will describe different numerical methods we used to explore the non-trivial fixed point solution of equation (3.12), including the special case $b=1$ (3.14) and several other expansions with different parameter $b$.

\subsubsection{Polynomial Expansion of the Potential $u(\rho)$ About the Origin}

It has been suggested $[167,168,169,170]$ that one can expand the effective potential as polynomials [155]. We first Taylor expand the potential $u(\rho)$ about the origin to order $m$

$$
u(\rho)=\sum_{n=0}^{m} \frac{1}{n !} \lambda_{n} \rho^{n} .
$$

Inserting equation (3.17) into left hand side of equation (3.16), we will get a polynomial of order $2 m$ in terms of $\rho$

$$
\begin{aligned}
& f_{0}\left(\lambda_{0}, \lambda_{1}, \cdots, \lambda_{m}\right)+f_{1}\left(\lambda_{0}, \lambda_{1}, \cdots, \lambda_{m}\right) \rho, \\
+ & f_{2}\left(\lambda_{0}, \lambda_{1}, \cdots, \lambda_{m}\right) \rho^{2}+\cdots+f_{2 m}\left(\lambda_{0}, \lambda_{1}, \cdots, \lambda_{m}\right) \rho^{2 m}=0,
\end{aligned}
$$

where all the coefficients $f_{i}$ s are in general functions of $\lambda_{0}, \cdots, \lambda_{m}$. The fixed point condition corresponds to setting all the coefficients of $\rho$ to zero. Since we only expand $u(\rho)$ to order $m$ and the $u^{\prime}$ and $\rho u^{\prime \prime}$ terms bring the power down by 1 , we can only impose the condition that $f_{0}=0, f_{1}=0, \cdots, f_{m-1}=0$. There are $m+1$ variables and $m$ independent equations so that we need to impose one more condition in order to solve the system. In practice, we notice that $f_{i}$ only depends on $\lambda_{0}, \cdots, \lambda_{i+1}$ (this is very easy to understand by looking at equation (3.16): the $u^{\prime}$ and $\rho u^{\prime \prime}$ bring the power down by 1). This nice property enable us to solve the equation iteratively and express all the $\lambda_{\mathrm{s}}$ in terms of $\lambda_{1}$. We can get $\lambda_{0}$ as a function of $\lambda_{1}$ from the condition $f_{0}=0$. And then we can substitute $\lambda_{0}$ by a 
function of $\lambda_{1}$ in $f_{1}, \cdots, f_{m-1}$. We can further get $\lambda_{2}$ as a function of $\lambda_{1}$ from $f_{1}=0$ condition. By doing so, we get the following relations

$$
\begin{aligned}
& \lambda_{0}=\frac{1}{3 \lambda_{1}+3} \\
& \lambda_{2}=-\frac{2}{3} \lambda_{1}\left(\lambda_{1}+1\right)^{2}, \\
& \lambda_{3}=\frac{2}{15} \lambda_{1}\left(\lambda_{1}+1\right)^{3}\left(13 \lambda_{1}+1\right), \\
& \lambda_{4}=-\frac{8}{7} \lambda_{1}^{2}\left(\lambda_{1}+1\right)^{4}\left(7 \lambda_{1}+1\right), \\
& \lambda_{5}=\frac{16}{189} \lambda_{1}^{2}\left(\lambda_{1}+1\right)^{5}\left(623 \lambda_{1}^{2}+121 \lambda_{1}+2\right), \\
& \vdots
\end{aligned}
$$

We further apply the boundary condition $\lambda_{m}=0$ in order to get the value of $\lambda_{1}$. Since $\lambda_{m}$ is a polynomial of $\lambda_{1}$, we will get multiple solutions from $\lambda_{m}=0$ condition. The fixed point value corresponds to the stable one with increasing $m$. See Figure (3.2).

Once we get the fixed point solution, we perturb around the fixed point and get the stability matrix and its the eigenvalues. The negative eigenvalue $\lambda_{1}$ relates to the critical exponent $\nu, \nu=-1 / \lambda_{1}$, and the smallest positive eigenvalue corresponds to the first sub-leading critical exponent $\omega$.

The stability matrix $M_{i j}$ is defined as

$$
M_{i j}=\frac{\partial f_{i}}{\partial \lambda_{j}},
$$

where $i$ and $j$ run from 0 to $m$. The matrix $M$ can be calculated from numerical derivative for large $m$. The method described above (expanding around the origin) is not the most efficient way to get the fixed point since the critical values or the fixed point values oscillate around the asymptotic value when we increase the expansion order $m$. This can be seen from Figure (3.3). In fact, we can only get 8 significant digits with order up to $m=80$. This is clearly not practical for high precision calculations. Therefore, a new method must be introduced. 


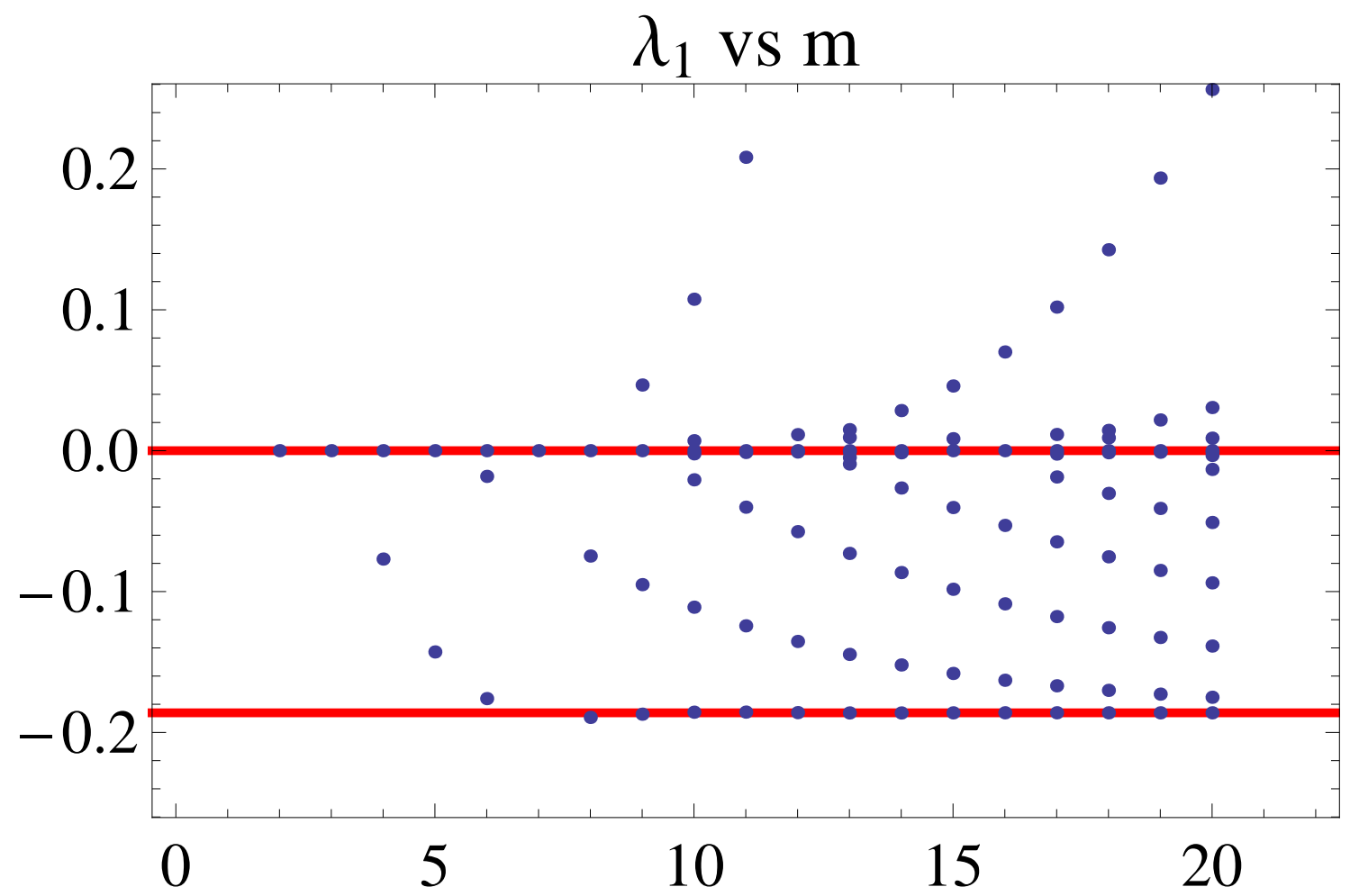

Figure 3.2: Two $\lambda_{1}$ s are stable with increasing polynomial order $m$.

\subsubsection{Polynomial Expansion of the Potential $u(\rho)$ About the Minimum}

It turns out that the convergence is much faster if we use the following expansion Equation (3.21)

$$
u(\rho)=\sum_{n=1}^{m} \frac{1}{n !} \lambda_{n}^{\prime}\left(\rho-\rho_{\text {min }}\right)^{n},
$$

where $\rho_{\min }$ corresponds to the minimum of $u(\rho)$ (See Figure (3.4)) and the expansion coefficients $\lambda_{n}^{\prime}$ are in general different from the coefficients used in Eq. (3.17).

The $\rho_{\min }$ in Eq. 3.21 can be calculated from the method described in the previous section since we do not need very high accuracy for it. The boundary condition can be chosen such that $\lambda_{m-1}^{\prime}=0$ and $\lambda_{m}^{\prime}=0$. All the $\lambda^{\prime}$ s are implicitly 


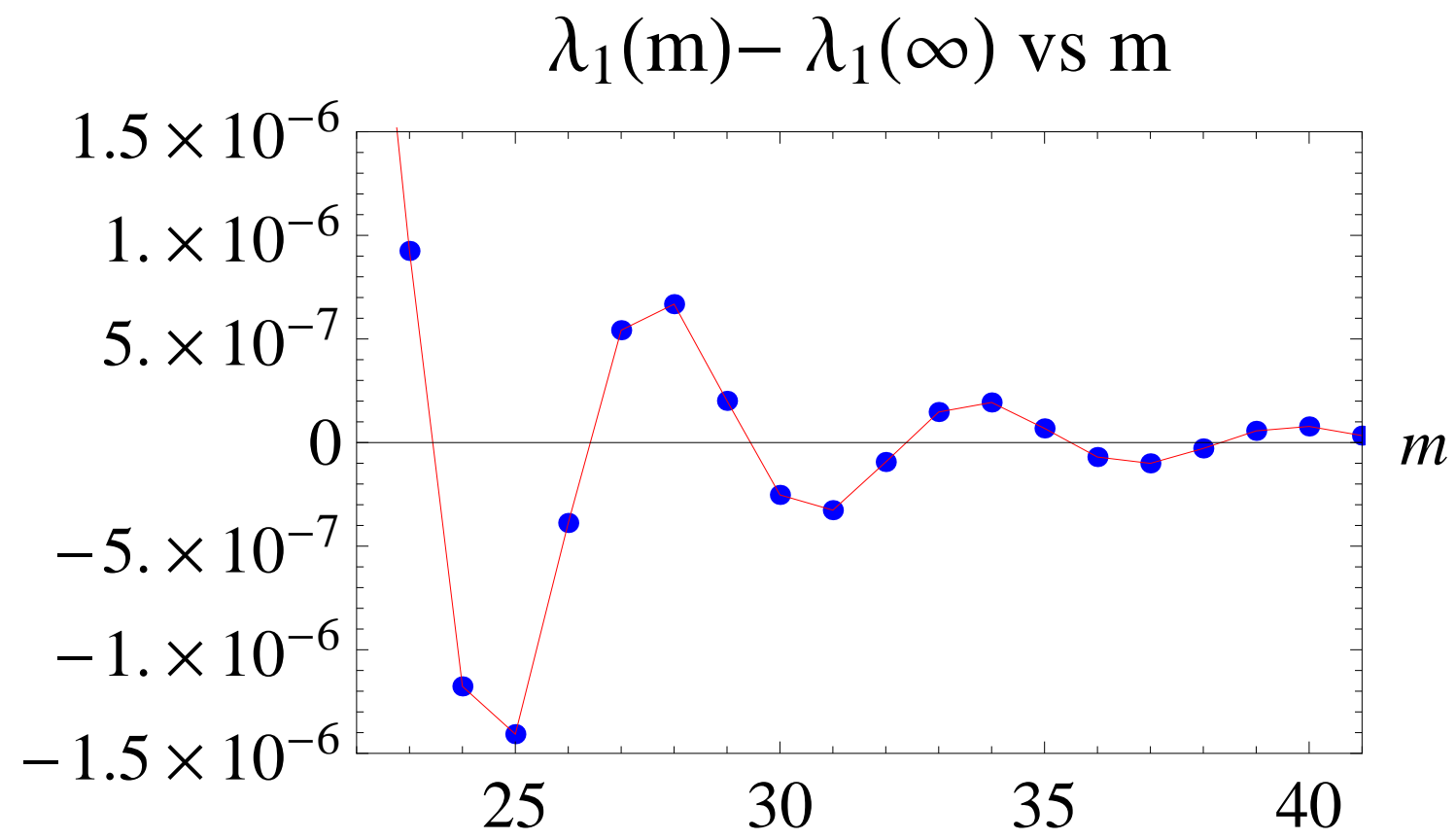

Figure 3.3: $\lambda_{1}$ approaches the asymptotic value $\lambda_{1}(\infty)=-0.18606424947 \cdots$ slowly with increasing polynomial order $m$ by using the expansion around the origin method.

dependent on $\lambda_{1}$ and $\lambda_{2}$. We can express $\lambda_{m-1}^{\prime}$ and $\lambda_{m}^{\prime}$ as functions of $\lambda_{1}$ and $\lambda_{2}$

$$
\begin{gathered}
\lambda_{m-1}^{\prime}=f_{1}\left(\lambda_{1}^{\prime}, \lambda_{2}^{\prime}\right)=0, \\
\lambda_{m}^{\prime}=f_{2}\left(\lambda_{1}^{\prime}, \lambda_{2}^{\prime}\right)=0 .
\end{gathered}
$$

The solution of Eq. (3.22) can be obtained from the two dimensional Newton method. The result is shown in Table (3.1). The method converges fast with increasing polynomial order $m$, see Fig. (3.5). The first six critical exponents are listed in Table 3.2.

We also tried the expansion slightly away from the potential minimum $\rho_{\min }$ and get similar results 


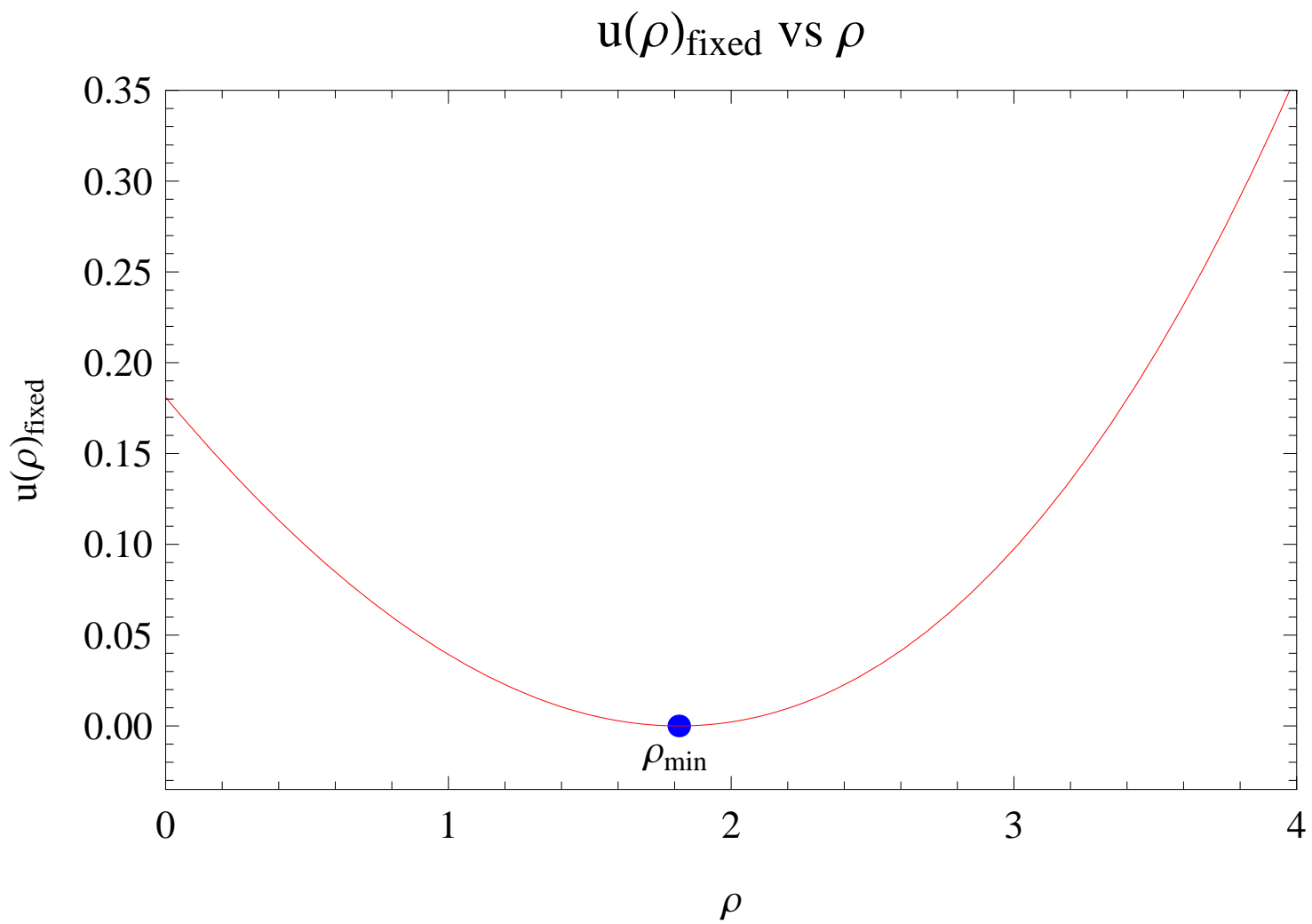

Figure 3.4: $u(\rho)$ versus $\rho$. $\rho_{\text {min }}$ corresponds to the minimum of the potential $u(\rho)$.

\begin{tabular}{rr}
\hline \hline \multicolumn{2}{c}{ Fixed point couplings } \\
\hline$\lambda_{1}^{\prime}$ & $-3.92436863865 * 10^{-14}$ \\
$\lambda_{2}^{\prime}$ & 0.126164421218 \\
$\lambda_{3}^{\prime}$ & 0.0298149647677 \\
$\lambda_{4}^{\prime}$ & 0.00626281638385 \\
$\lambda_{5}^{\prime}$ & -0.000275905517003 \\
$\lambda_{6}^{\prime}$ & -0.00112762344235 \\
\hline
\end{tabular}

Table 3.1: The first six couplings at the fixed point of Eq. (3.14) for the expansion of Eq. (3.21) with $m=45$ and $\rho_{\min }=1.814898403687$. 


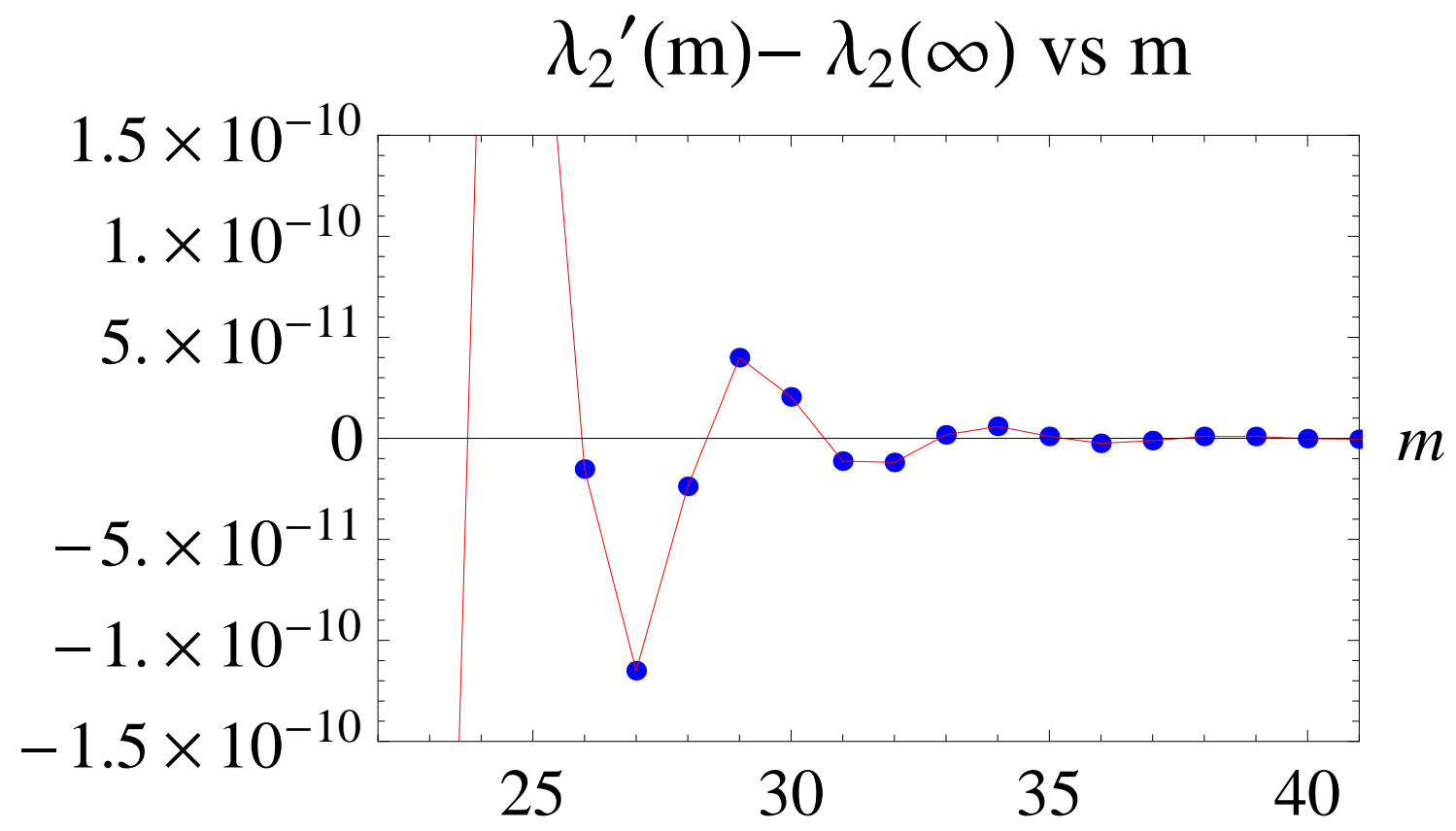

Figure 3.5: $\lambda_{2}^{\prime}$ approaches the asymptotic value very fast with in creasing polynomial order $m$ by using the expansion around the potential minimum method.

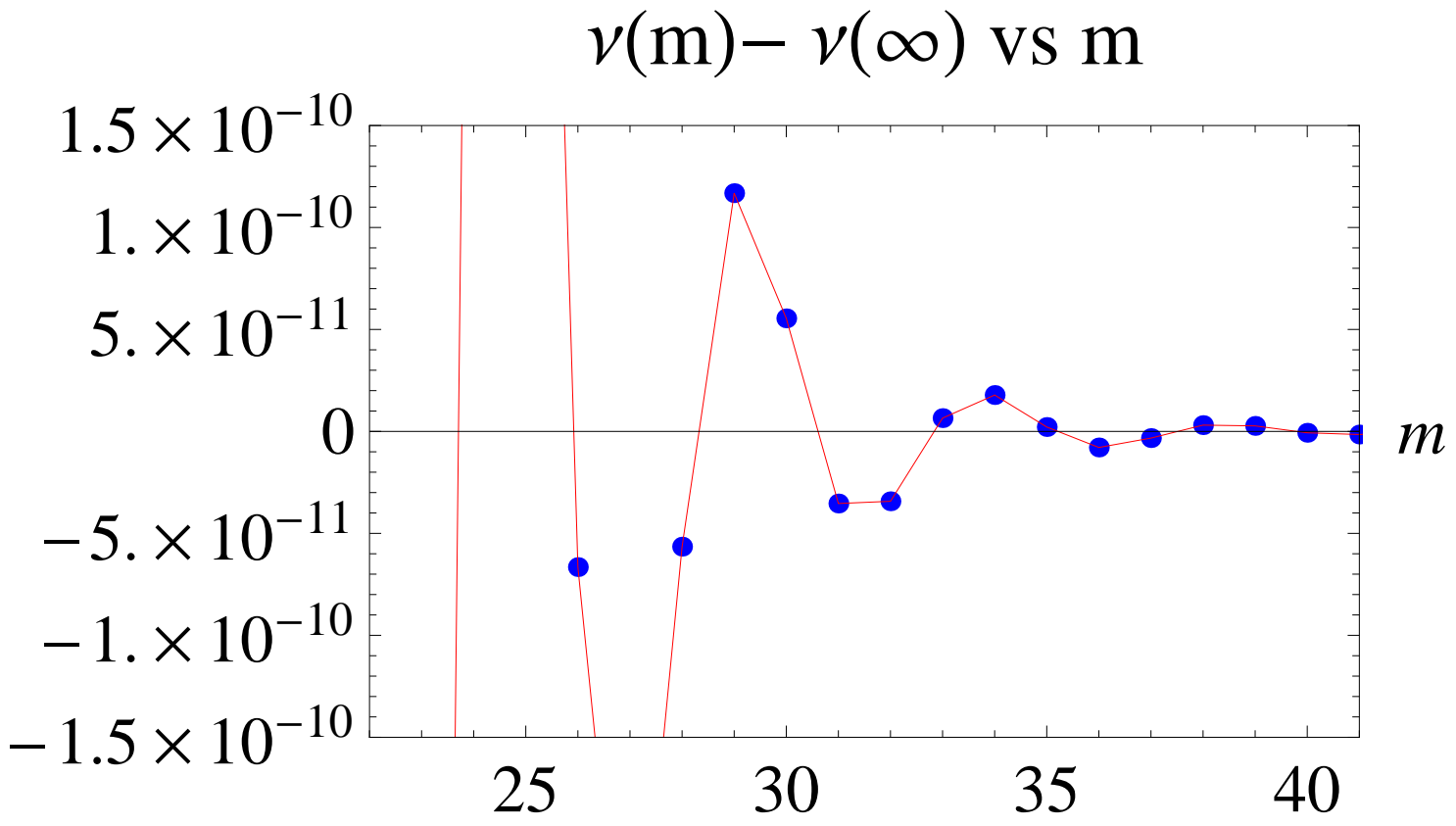

Figure 3.6: The convergence of the critical exponent $\nu$. 


\begin{tabular}{rr}
\hline \hline Critical exponents \\
\hline$\nu$ & 0.64956177388 \\
$\omega$ & 0.6557459391 \\
$\omega_{2}$ & 3.18000651 \\
$\omega_{3}$ & 5.9122305 \\
$\omega_{4}$ & 8.796093 \\
$\omega_{5}$ & 11.79808 \\
\hline
\end{tabular}

Table 3.2: The first six critical exponents calculated from the expansion of Eq. (3.21) with $m=42$ and $\rho_{\min }=1.814898403687$.

\subsection{Equivalence between Litim and Wilson- Polchinski equation}

Before discussing the critical properties of the flow equation under other different cutoff functions, let us look more closely at the Wilson-Polchinski flow equation. Felder [133] has shown that the hierarchical RG is equivalent to the WilsonPolchinski RG in the continuous blocking spin limit, i.e., $l^{D} \rightarrow 1$ limit. Moreover, Morris [154] proved that the ERGE with optimized cutoff function is also equivalent to the Wilson-Polchinski RG.

If we rewrite the Eq. 3.8 in terms of the variable $v(\phi)=V_{k}(\phi) / k^{d}$ and $\psi=$ $\phi / k^{(2-d) / 2}$, we obtain the following flow equation for $d=3$

$$
\partial_{t} v=-3 v+\frac{1}{2} \varphi v^{\prime}+\frac{1}{1+v^{\prime \prime}}
$$

The flow equation for the Wilson-Polchinski flow equation in terms of $v(\psi)_{w}$ is

$$
\partial_{t} v_{w}=-3 v_{w}+\frac{1}{2} \varphi_{w} v_{w}^{\prime}-v_{w}^{\prime \prime}+v_{w}^{\prime 2}
$$

In this section, I will show how the above two equations 3.23 and 3.24 are equivalent. 
The potential $v(\varphi)$ in (3.23) is related to $v_{w} \varphi_{w}$ in (3.24) by a Legendre transformation

$$
\begin{gathered}
v(\varphi)=v_{w}\left(\varphi_{w}\right)-\frac{1}{2}\left(\varphi_{w}-\varphi\right)^{2} . \\
\varphi=\varphi_{w}-v_{w}^{\prime}\left(\varphi_{w}\right)
\end{gathered}
$$

Equation 3.25 corrects a typo in Eq. (9) of [155].

We can further get the relationship between the derivative of $v$ and the that of $v_{w}$

$$
\begin{aligned}
v^{\prime} & =\frac{\partial v}{\partial \varphi} \\
& =\frac{\partial v_{w}}{\partial \varphi_{w}} \frac{\partial \varphi_{w}}{\partial \varphi}-\left(\varphi_{w}-\varphi\right)\left(\frac{\partial \varphi_{w}}{\partial \varphi}-1\right) \\
& =v_{w}^{\prime} \frac{1}{1-v_{w}^{\prime \prime}}-v_{w}^{\prime} \frac{v_{w}^{\prime \prime}}{1-v_{w}^{\prime \prime}} \\
& =v_{w}^{\prime}, \quad v^{\prime \prime}=\frac{\partial v_{w}^{\prime}}{\partial \varphi}=v_{w}^{\prime \prime} \frac{\partial \varphi_{w}}{\partial \varphi}=v_{w}^{\prime \prime} \frac{1}{1-v_{w}^{\prime \prime}},
\end{aligned}
$$

and further

Finally,

$$
\frac{1}{1+v^{\prime \prime}}=\frac{1}{1+\frac{v_{w}^{\prime \prime}}{1-v_{w}^{\prime \prime}}}=1-v_{w}^{\prime \prime} \text {. }
$$

$$
\begin{aligned}
& -3 v+\frac{1}{2} \varphi v^{\prime}+\frac{1}{1+v^{\prime \prime}} \\
= & -3\left(v_{w}-\frac{1}{2} v_{w}^{\prime \prime 2}\right)+\frac{1}{2}\left(\varphi_{w}-v_{w}^{\prime}\right) v_{w}^{\prime}+\left(1-v_{w}^{\prime \prime}\right) \\
= & -3 v_{w}+\frac{1}{2} \varphi_{w} v_{w}^{\prime}+v_{w}^{\prime 2}-v_{w}^{\prime \prime}+1 \\
= & -3 v_{w}+\frac{1}{2} \varphi_{w} v_{w}^{\prime}-v_{w}^{\prime \prime}+v_{w}^{\prime 2}+1 .
\end{aligned}
$$

Since at the fixed point $\partial_{t} v^{\prime}=0$, the extra constant 1 plays no role on the fixed point equation. 


\subsection{Effects Of The Cutoff Functions}

In Sec. 3.3, Eq. 3.13 and 3.14 are derived after proper field rescaling. This can be understood from App. B. In this section, we will use the properties derived in App. B to expand the flow equation and analyze the cutoff dependence of the critical exponents.

The "wrong" flow equation stated in [166] Eq. (2) is

$$
-3 u+\rho u^{\prime}+\int_{0}^{\infty} d y \frac{-y^{\frac{3}{2}} r^{\prime}(y)}{y(1+r)+u^{\prime}+2 \rho u^{\prime \prime}}=0,
$$

with $r(y)=b\left(\frac{1}{y}-1\right) \theta(1-y)$, where $\theta(x)$ is Heaviside step function.

After integrating over $y$, Eq. (3.32) becomes

$$
-3 u+\rho u^{\prime}+\frac{2 b}{\sqrt{(b-1)(b+w)}} \operatorname{ArcTanh} \sqrt{\frac{b-1}{b+w}}=0 \quad \text { for } \quad b>1 .
$$

By applying App. B "No A Dependence" Theorem, we can remove the prefactor $\frac{2 b}{\sqrt{b-1}} \Rightarrow$

$$
-3 u+\rho u^{\prime}+\frac{1}{\sqrt{b+w}} \operatorname{ArcTanh} \sqrt{\frac{b-1}{b+w}}=0 .
$$

Expanding $\operatorname{ArcTanh}(x)=x+\frac{x^{3}}{3}+\frac{x^{5}}{5}+\cdots$, the ArcTanh dependent term will become

$$
\begin{aligned}
& \frac{1}{\sqrt{b+w}}\left(\sqrt{\frac{b-1}{b+w}}+\frac{1}{3}\left(\sqrt{\frac{b-1}{b+w}}\right)^{3}+\cdots\right. \\
= & \sqrt{b-1}\left(\frac{1}{b+w}+\frac{1}{3} \frac{b-1}{(b+w)^{2}}+\frac{1}{5} \frac{(b-1)^{2}}{(b+w)^{3}}+\cdots\right. \\
\stackrel{\text { No A dependence }}{\longrightarrow} & \frac{1}{b+w}+\frac{1}{3} \frac{b-1}{(b+w)^{2}}+\frac{1}{5} \frac{(b-1)^{2}}{(b+w)^{3}}+\cdots \\
\Rightarrow & \frac{1}{b\left(1+\frac{w}{b}\right)}+\frac{1}{3} \frac{b-1}{b^{2}\left(1+\frac{w}{b}\right)^{2}}+\cdots \\
\stackrel{\text { No A dependence }}{\longrightarrow} & \frac{1}{1+\frac{w}{b}}+\frac{1}{3} \frac{b-1}{b} \frac{1}{\left(1+\frac{w}{b}\right)^{2}}+\frac{1}{5}\left(\frac{b-1}{b}\right)^{2} \frac{1}{\left(1+\frac{w}{b}\right)^{3}} \cdots \\
\stackrel{\text { No B dependence }}{\longrightarrow} & \frac{1}{1+w}+\frac{1}{3} \frac{b-1}{b} \frac{1}{(1+w)^{2}}+\frac{1}{5}\left(\frac{b-1}{b}\right)^{2} \frac{1}{(1+w)^{3}}+\cdots .
\end{aligned}
$$


Note that if one "accidentally" used $y^{5 / 2}$ instead of $y^{3 / 2}$, i.e.,

$$
-3 u+\rho u^{\prime}+\int_{0}^{\infty} d y \frac{-y^{\frac{5}{2}} r^{\prime}(y)}{y(1+r)+u^{\prime}+2 \rho u^{\prime \prime}}=0,
$$

then after integration one will get

$$
-3 u+\rho u^{\prime}+\frac{2 b\left(\sqrt{\frac{b+w}{b-1}} \operatorname{ArcTanh} \sqrt{\frac{b-1}{b+w}}-1\right)}{b-1}=0 \text { for } \quad b>1 .
$$

For $y^{3 / 2}$ we will have

$$
-3 u+\rho u^{\prime}+\frac{2 b}{b-1} x\left(x+\frac{x^{3}}{3}+\frac{x^{5}}{5}+\cdots\right)=-3 u+\rho u^{\prime}+\frac{2 b}{b-1}\left(x^{2}+\frac{x^{4}}{3}+\frac{x^{6}}{5}+\cdots\right),
$$

and for $y^{5 / 2}$ we will have

$$
-3 u+\rho u^{\prime}+\frac{2 b}{b-1}\left[\frac{1}{x}\left(x+\frac{x^{3}}{3}+\frac{x^{5}}{5}+\cdots\right)-1\right]=-3 u+\rho u^{\prime}+\frac{2 b}{b-1}\left(\frac{x^{2}}{3}+\frac{x^{4}}{5}+\cdots\right),
$$

where $x=\sqrt{\frac{b-1}{b+w}}$.

Therefore, we can see that the difference between $y^{5 / 2}$ and $y^{3 / 2}$ is just in the coefficients of the $x^{2 n}$. From Eq. 3.39, one can also see that one can start with Eq. 3.16 and consider higher orders of $x^{2}=\frac{b-1}{b+w}$ as perturbations. The first step is to add a $\epsilon x^{4}$ to Eq. 3.16. For $b=1$ case, we add $\epsilon \frac{1}{1+w}$. One will find that the critical exponents $\nu$ and $\omega$ change with different $\epsilon$, see Fig. 3.7. If one plot them in the $\omega-\frac{1}{\nu}$ plane, one would get Fig. 3.8

We can also calculate critical exponents from Eq. 3.34 and 3.38 directly for different values of parameter $b$. Parts of the results are shown in Fig. 3.9 and 3.10. From Fig. 3.9 we can see that the $y^{3 / 2}$ results, which corresponds to the Eq. 3.32, converges to the "exact" Wilson-Polchinski RG result but not the $y^{5 / 2}$. The possible explanations are that either $y^{5 / 2}$ result "jump" to the "exact" result in the $b \rightarrow 1$ limit or $y^{3 / 2}$ is the proper form for the optimized cutoff ERG equation. I have not re-derived the Eq. 3.8 and 3.9 but prefer the later explanation. 
$v$ and $\omega$ VS $\epsilon$

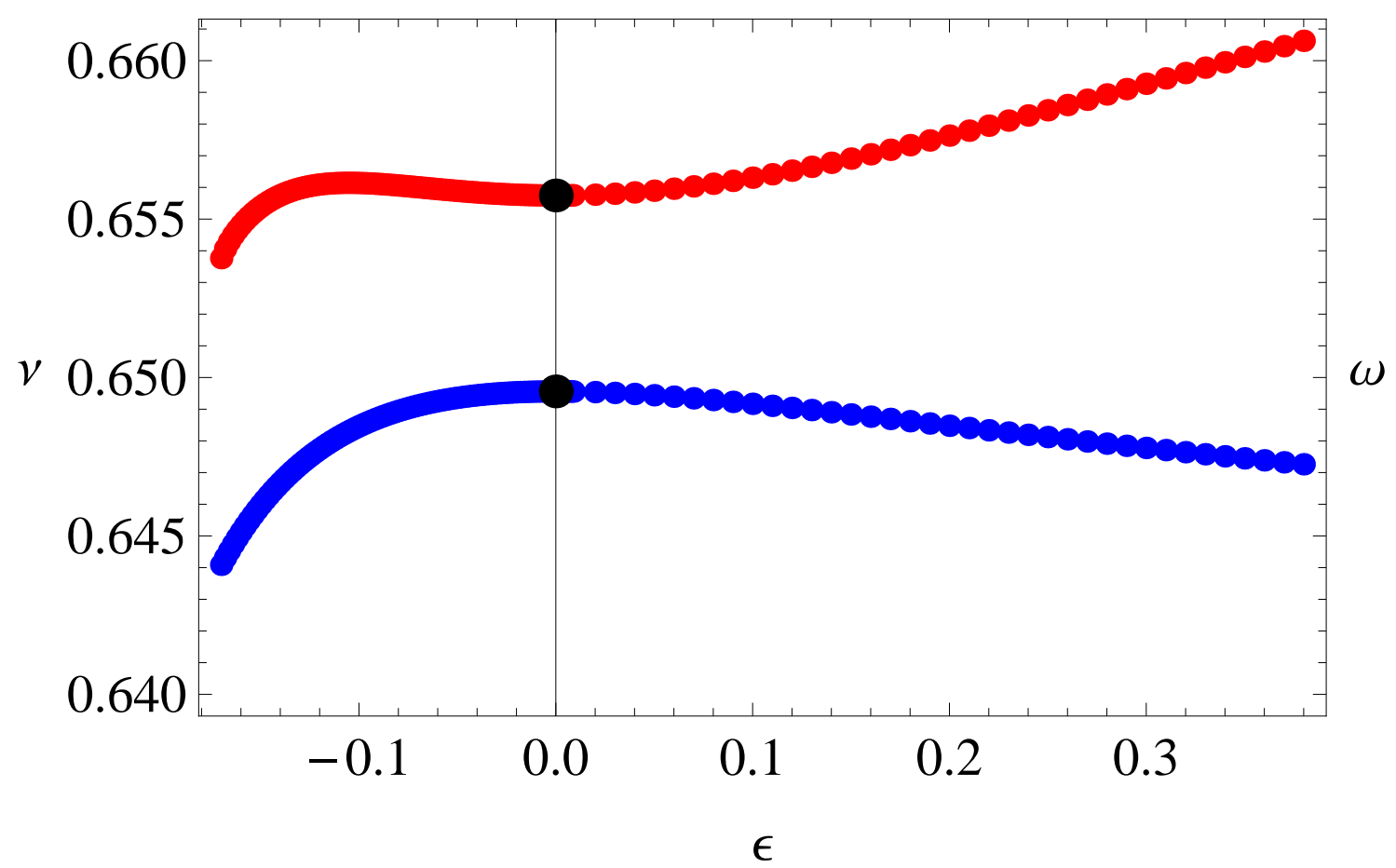

Figure 3.7: Critical exponents $\nu$ and $\omega$ change as function of $\epsilon$. 


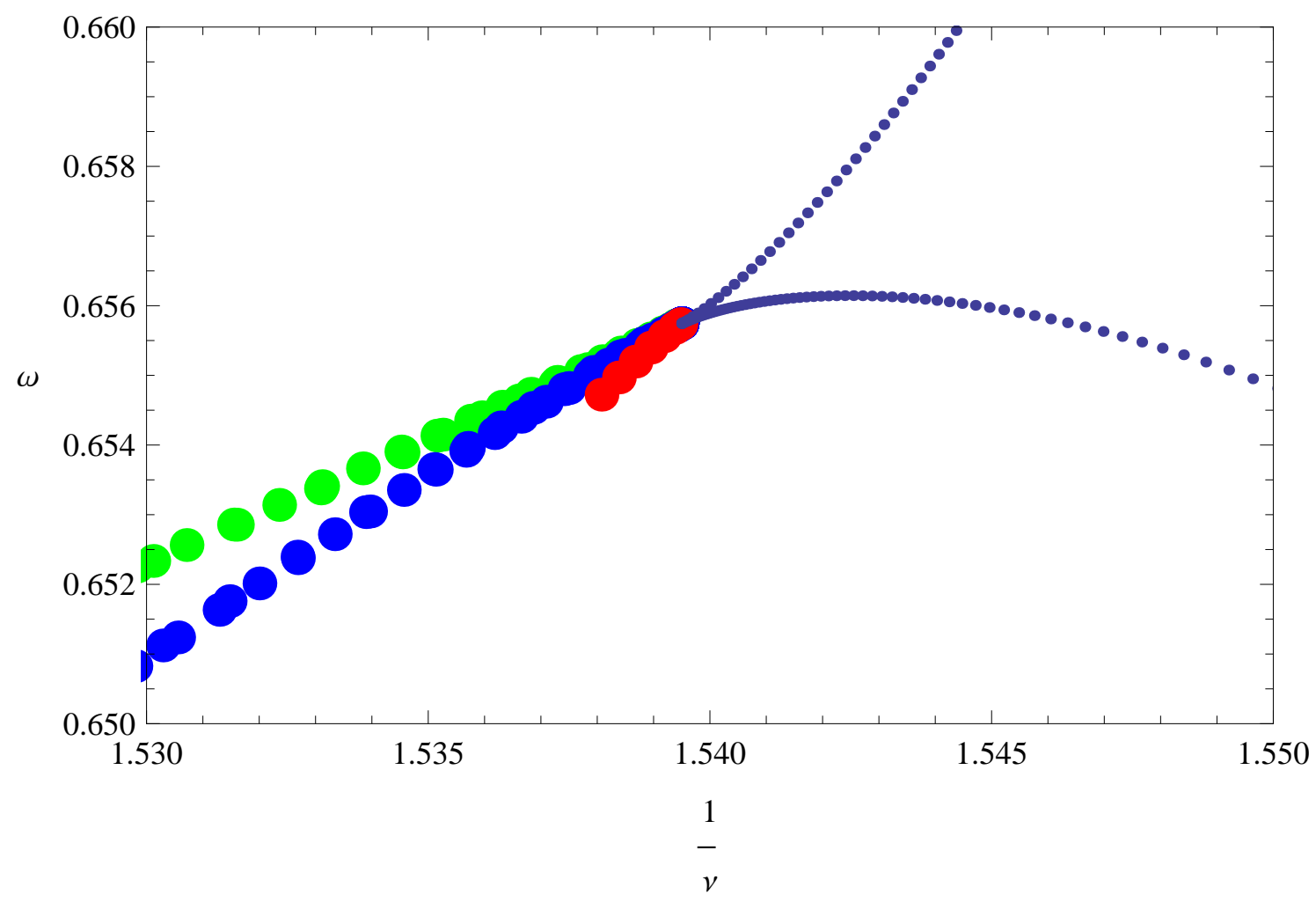

Figure 3.8: Critical exponents $\nu$ and $\omega$ for different $\epsilon$.

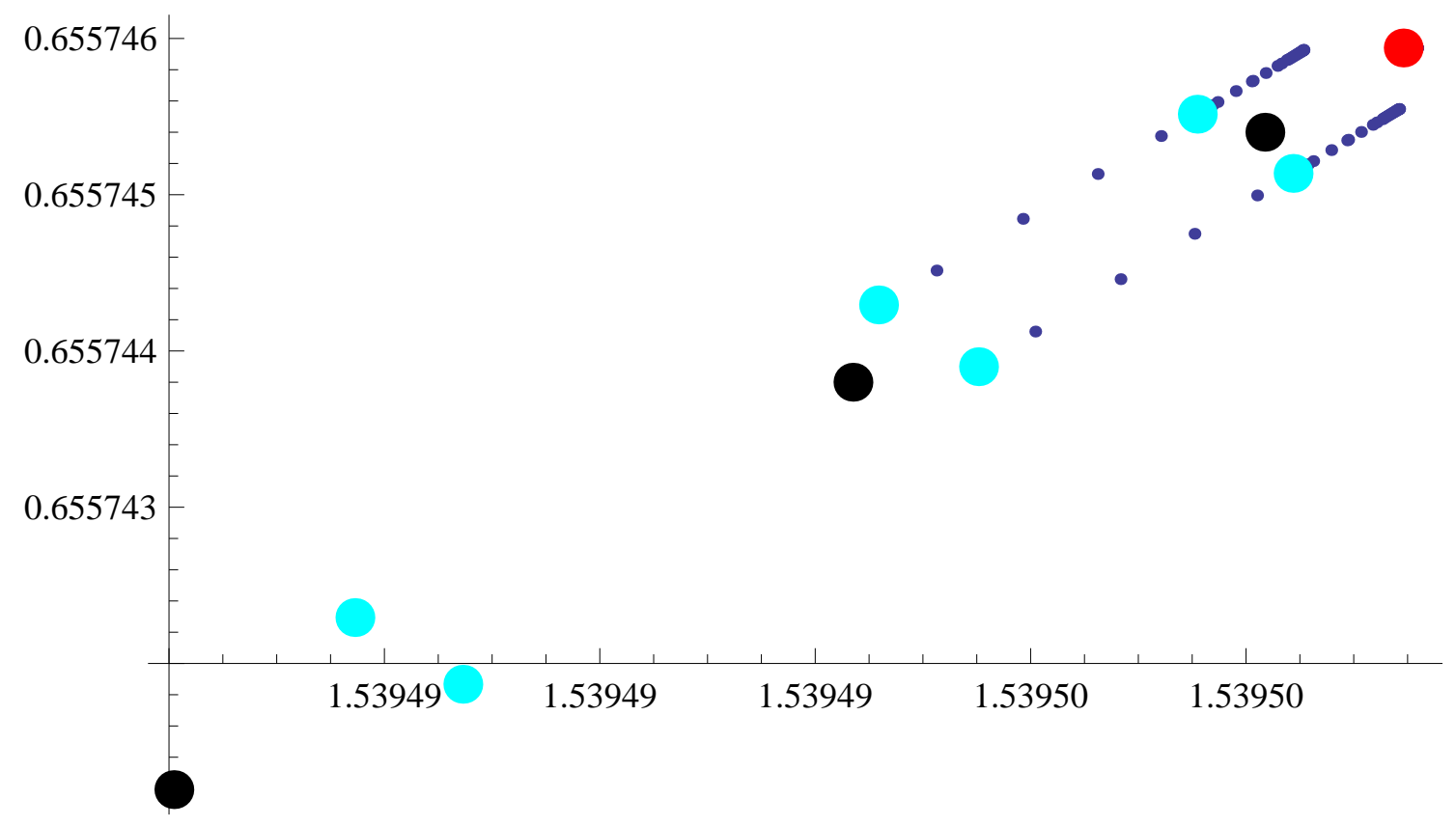

Figure 3.9: Show that Litim used formula corresponding to $y^{5 / 2}$ instead of $y^{3 / 2}$ to get the data "DataNuOmega.m". 


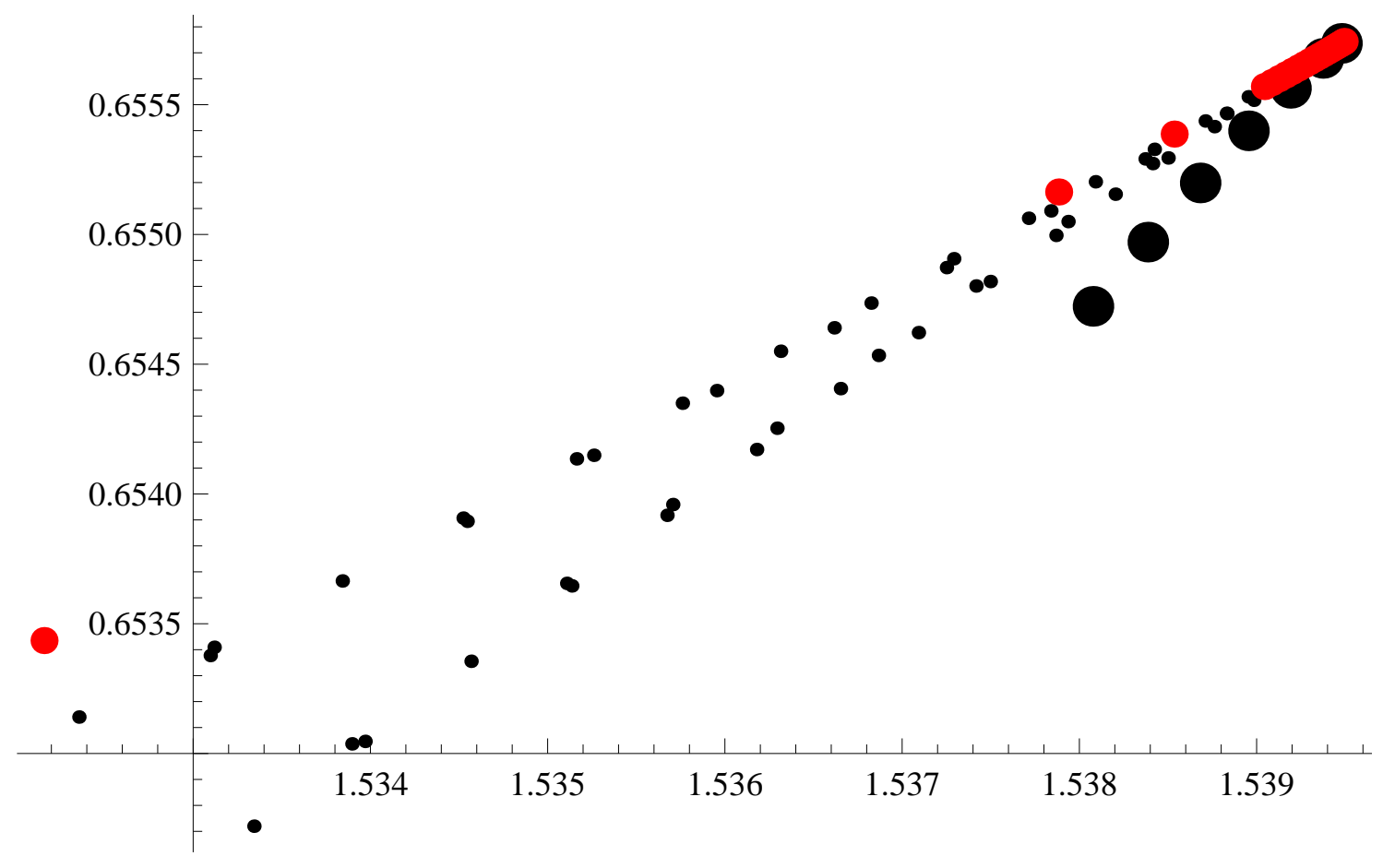

Figure 3.10: $\mathrm{y}$ axis is the $\omega$ and $\mathrm{x}$ axis is the $1 / \nu$. 


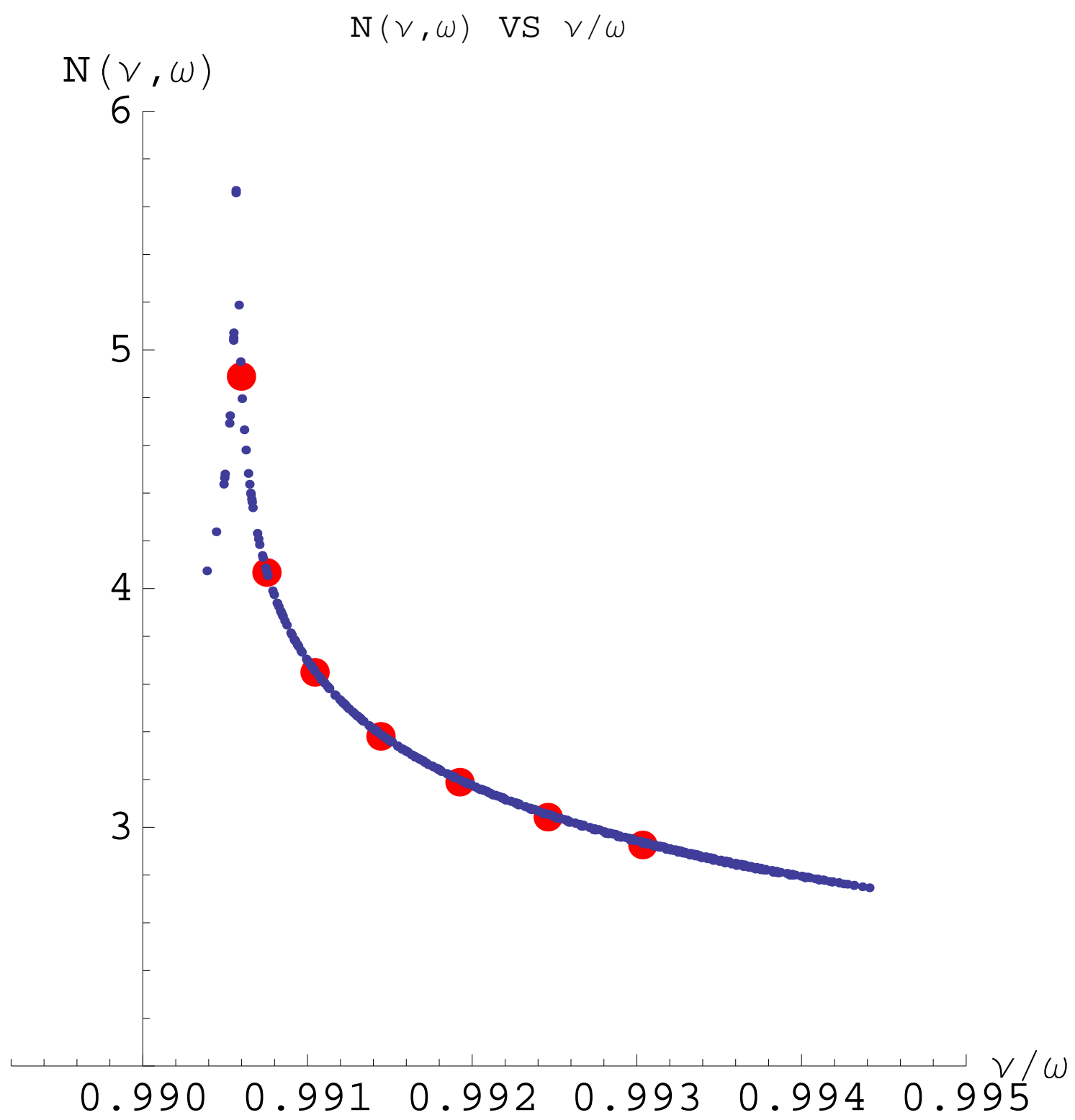

Figure 3.11: Blue points calculated with different $\epsilon_{1}$ and $\epsilon_{2}$; red points are calculated from the hierarchical model with different $b^{D}$. 


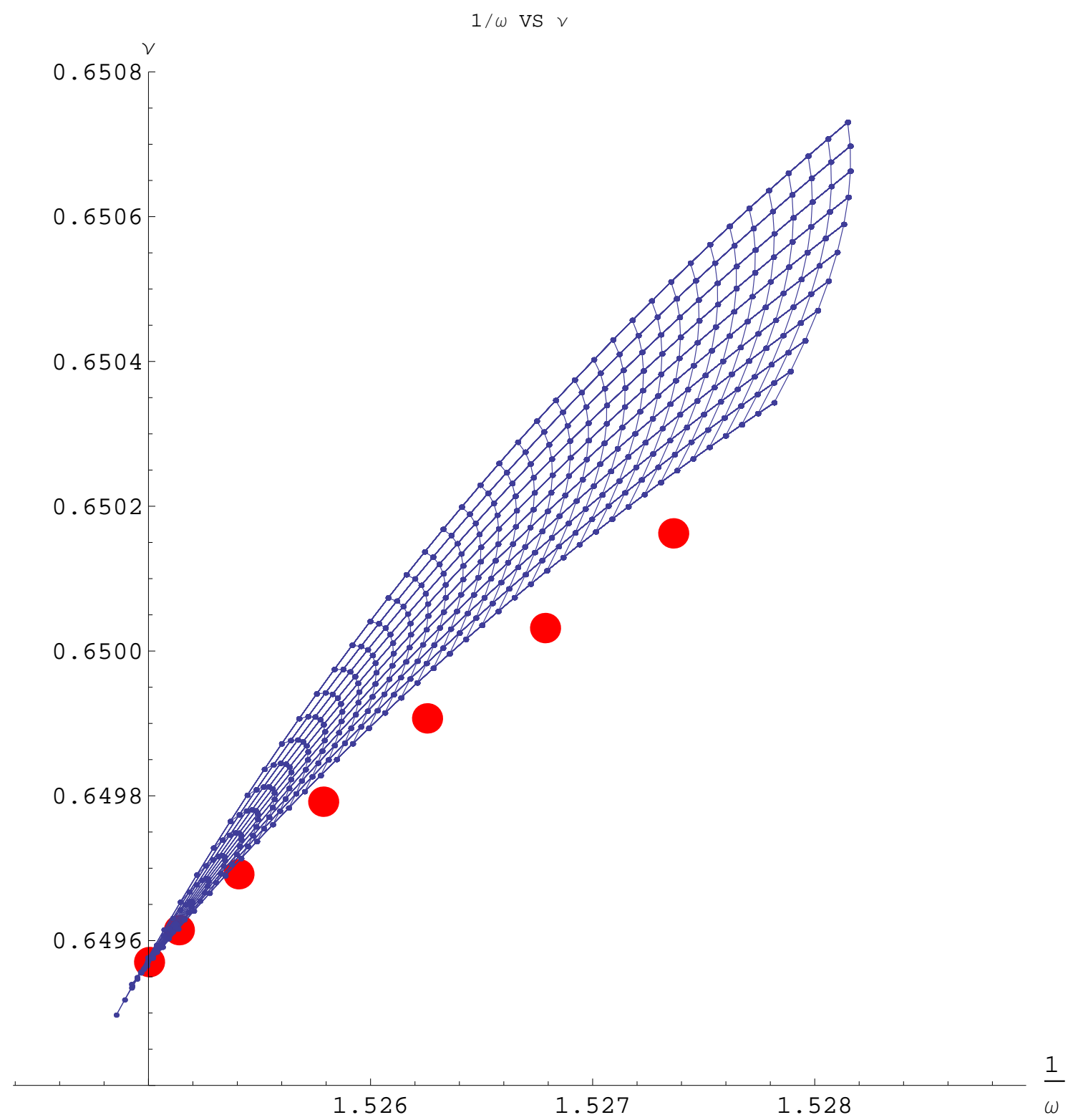

Figure 3.12: Blue points calculated with different $\epsilon_{1}$ and $\epsilon_{2}$; red points are calculated from the hierarchical model with different $b^{D}$. 


\section{CHAPTER 4 RENORMALIZATION GROUP AND LATTICE GAUGE THEORIES}

\subsection{Motivations}

In the previous chapters, we have been focusing on the classical spin models. We detailed the properties of the partition function zeros, Lee-Yang zeros and Fisher zeros, the finite size scaling (FSS) technique, their relations to the critical exponents, discrete and continuous block-spinning, two lattice matching, $\beta$ functions, complex RG flows, and ERGE. In the following two chapters, we will apply the technique developed and switch our attention to the more complicated and more physically important lattice gauge models. In this chapter, I will focus on the pure gauge $S U(2)$ lattice gauge model.

As stated in Sec. 2.2.8, FSS is a powerful tool to extrapolate information from finite system to the thermodynamic limit. It has also be used to study the critical behavior of the system, such as calculating critical exponents. The essence of FSS is the following: when the system is close to the second order phase transition, long distance properties are only determined by some global features of the system, such as the internal symmetries, the dimensionality, and the range of the interaction. The details of the Hamiltonian interactions is not so important compared to those global feature. Universality further enables us to classify seemingly different systems into certain classes. Connection between spin models and lattice gauge models can be seen from the conjecture of Svetitsky and Yaffe [171]. They pointed out that, for $d+1$ dimensional $S U(N)$ pure gauge model, "if any portion of the boundary is second-order, then the critical behavior will be described by some fixed point of $d$-dimensional, $Z(N)$ invariant spin systems."

For a pure gauge $S U(2)$ theory in $3+1$ dimensions, we expect the universality class of the finite temperature transition to be the same as the 3 dimensional Ising 
model. Existing results on FSS for $S U(2)[172,173,174,175,176]$ agree well with this expectation. The above conjecture was based on the assumption that the effective spin system of the gauge theory has only short range interactions. However, there were indications that the effective interaction of the Polyakov loops might be long range [177]. The simulation of $S U(2)$ gauge theory in $2+1$ dimensions [178] also showed that the critical exponent $\beta(0.2 \pm 0.04)$ deviates from the corresponding $2 \mathrm{D}$ Ising exponent(0.125). In this chapter, we would like use FSS to analyze the lattice gauge model and try to extrapolate the critical exponents $\nu$ and $\omega$ and compare those with the three dimensional nearest neighbor Ising model.

\subsection{Introduction To Lattice Gauge Theory}

Classical lattice spin models are familiar to physicists because they are reflections of the lattice structures in materials in the laboratory. Some spin models have been extensively discussed in the previous chapters. Here we are going to discuss some more abstracted systems. In particular, we are going to use space-time lattice to describe the gauge field theory. The goal is to construct solutions of cutoff theories which can be mapped onto the real continuous Minkowski space-time field theory. The trick is to avoid integrating over infinite number of degrees of freedom. One can convert the original field theory problems to statistical mechanics ones.

Non-Abelian gauge theories in four dimensions were discovered to be asymptotically free (AF) $[10,11]$. Roughly speaking, AF means that the coupling "constant" $g(\Lambda)$ decreases with increasing $\Lambda$ and vanishes as $\Lambda \rightarrow \infty$. In another word, the effective coupling $g$ goes to zero at short distance. This has been tested extremely well in the deep-inelastic scattering of electrons or neutrinos off strongly interacting protons and neutrons. This supports the conjecture that the strong interaction can be described by the $S U(3)$ "color" gauge group with quarks in the fundamental representations. Later on, people conjectured that the quarks should 
be confined in such theories, which is consistent with the observation that no free quark has ever been catched in the experiment. Confinement is very natural in the lattice gauge theory in the strong coupling limit [179]. Strong coupling limit in the lattice gauge theory corresponds to the high temperature expansion in the spin system. In the lattice gauge theory, the effective bare coupling will go to zero as one takes the lattice spacing to the continuum, which corresponds to the low temperature domain. The task then would be to obtain a theory that has no phase transition when one goes from the high temperature to low temperature region. This way, one could then construct a computable theory that has the properties of both confinement and AF. However, we will see later on that many lattice gauge models have non-trivial phase transition in the intermediate region. On the one hand, one should make sure that one is in the 'right' phase if one wants to simulate the continuous physics. On the other hand, the phase structure of different lattice gauge theories are interesting on their own. In the following, we will see that the statistical mechanics of lattice gauge theories share many similarities with the classical spin models. More details about the lattice gauge theories and lattice QCD like theories are discussed in the App. C.

\subsection{Finite Size Scaling of The $S U(2)$ Lattice Gauge Theory}

In this section, we work on $S U(2)$ lattice gauge theory in $3+1$ dimensions: $N_{\tau} \times N_{\sigma}^{3}$. We fix the number of site in the time direction $N_{\tau}$ to be 4 , and vary the number of site in the space dimension $N_{\sigma}$ from $2,4,6,8, \cdots 16$. We use periodic boundary conditions in all the directions. We focus on direct estimations of the exponents $\nu$ and $\omega$. For $N_{\tau}=4$, the only direct estimate of $\nu$ we are aware of is 0.65(4), which was obtained in Ref. [172]. This is compatible with the accurate value $0.6298(5)$ obtained in Ref. [180] for the 3-dimensional Ising model. In this 
section, we would like to improve the accuracy of the estimate of Ref. [172] and resolve the corrections due to the irrelevant directions. The second aim is largely unexplored and a better understanding of these corrections could help us design methods to reduce these effects as done in Ref. [180] for the 3 dimensional Ising model. In the existing work for $N_{\tau}=4$ in Refs. $[172,173,176]$, a fixed $\beta$ interval procedure was used. This means that the $\beta$ interval is fixed for different volumes. In the following, we shrink the interval in order to reduce the nonlinear effects [181] and use a finer $\beta$ resolution.

\subsubsection{Binder cumulant and FSS}

We define the 4th order Binder cumulant [182], $g_{4}$, as

$$
g_{4}=1-\frac{\left\langle P^{4}\right\rangle}{3\left\langle P^{2}\right\rangle^{2}}, \quad P=\frac{1}{N_{\sigma}^{3}} \sum_{\boldsymbol{x}} \frac{1}{2} \operatorname{Tr} \prod_{\tau=1}^{N_{\tau}} U_{\tau, \boldsymbol{x} ; 0} .
$$

Related definitions appear in the literature, such as $B_{4}=\frac{\left\langle P^{4}\right\rangle}{\left\langle P^{2}\right\rangle^{2}}$. We assume that there is no external field and that the $g_{4}$ depends on the scaling variables as

$$
g_{4}=g_{4}\left(u_{\kappa} N_{\sigma}^{1 / \nu}, u_{1} N_{\sigma}^{-\omega}, \ldots\right)
$$

with

$$
\begin{aligned}
& u_{\kappa}=\kappa+u_{\kappa}^{(2)} \kappa^{2}+\ldots \\
& u_{1}=u_{1}^{(0)}+u_{1}^{(1)} \kappa+\ldots,
\end{aligned}
$$

where $u_{\kappa}$ is the only relevant scaling variable, $u_{1}$ is the first irrelevant scaling variable, and $\kappa$ is the reduced quantity $\kappa=\left(\beta-\beta_{c}\right) / \beta_{c}$. Figure 4.1 shows the Binder cumulant as function of $\beta$ for different volumes. We can see that as the volume increases, all the curves cross around one $\beta$, which is just the critical $\beta_{c}$. This can be understood by expanding the Binder cumulant up to the first nonlinear corrections. 


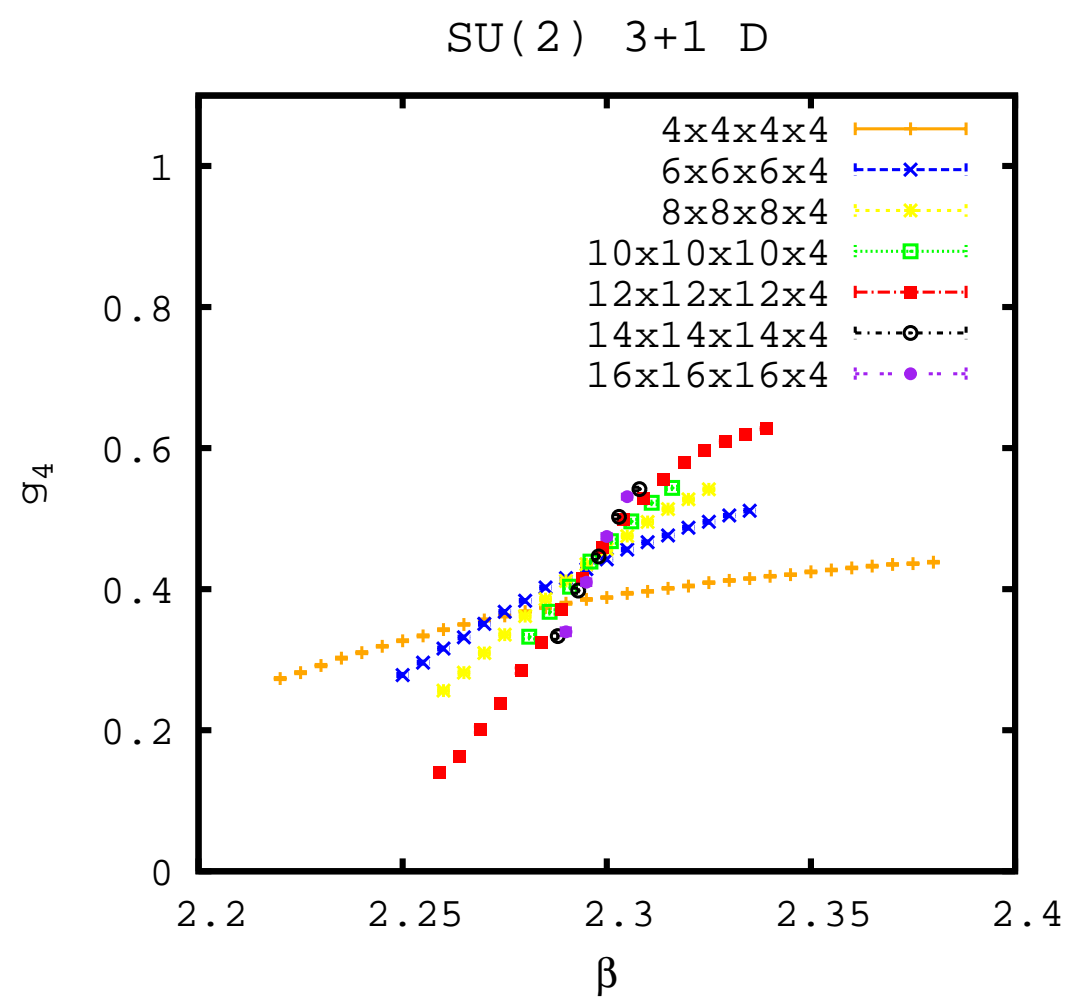

Figure 4.1: $g_{4}$ versus $\beta$ for different volumes.

We obtain

$$
g_{4}\left(\beta, N_{\sigma}\right)=g_{4}\left(\beta_{c}, \infty\right)+f_{1} \kappa N_{\sigma}^{1 / \nu}+f_{2} \kappa^{2} N_{\sigma}^{2 / \nu}+\left(c_{0}+c_{1} \kappa N_{\sigma}^{1 / \nu}\right) N_{\sigma}^{-\omega}+\cdots
$$

This expansion is accurate if $|\kappa| N_{\sigma}^{1 / \nu}$ is small enough. In addition we would like the nonlinear effects of $f_{2}$ and $c_{1}$ to be negligible. It is easy to estimate $f_{2}$ from numerical data for intermediate values of $\kappa[181]$. However, $c_{1}$ is more difficult to resolve from an already small effect and its effect will be ignored. In the following, we will work with values of $|\kappa| N_{\sigma}^{1 / \nu}$ such that the effects of $f_{2}$ are within the numerical errors of $g_{4}$ that we now proceed to discuss. 


\subsubsection{Determination of the critical exponent $\nu$}

We now focus on the estimation of $\nu$. If we are reasonably close to $\beta_{c}$, we can use the linear form:

$$
g_{4}\left(\beta, N_{\sigma}\right) \simeq g_{4}\left(\beta_{c}, \infty\right)+c_{0} N_{\sigma}^{-\omega}+f_{1} \kappa N_{\sigma}^{1 / \nu}
$$

This expression contains 6 unknown parameters: $g_{4}\left(\beta_{c}, \infty\right), c_{0}, \omega, f_{1}, \beta_{c}$ and $1 / \nu$ and we will use a new strategy to attack this difficult problem . A first observation is that the dependence on $\nu$ can be isolated from the other parameters by studying the linear dependence in $\beta$. However, one should keep in mind that the slope of this linear fit depends implicitly on the choice of the center of the interval which should be as close as possible to $\beta_{c}$. In order to guarantee that nonlinear effects are under control, we will consider $\beta$ intervals of the form

$$
\left|\beta-\left(\beta_{c}\right)_{a p p}\right|<0.015 \times\left(4 / N_{\sigma}\right)^{(1 / \nu)_{a p p}} .
$$

We will start with reasonable values for $\left(\beta_{c}\right)_{a p p}$ and $(1 / \nu)_{a p p}$ and then show that the effect of their variations is small. The factor 0.015 has been chosen following a procedure described in Ref. [181] and guarantees an approximate linear relation between $g_{4}$ and $\beta$ at fixed $N_{\sigma}$ :

$$
g_{4} \simeq a_{N_{\sigma}}+b_{N_{\sigma}} \times \beta
$$

This is illustrated in Fig. 4.2 for $\left(\beta_{c}\right)_{a p p}=2.299,(1 / \nu)_{a p p}=1.6$ and $N_{\sigma}=10$. We see that in the chosen interval the deviation from linearity seem mostly due to statistical errors rather than a systematic curvature that would be observed if we had chosen a broader interval. The volume dependence of the parameters of the 


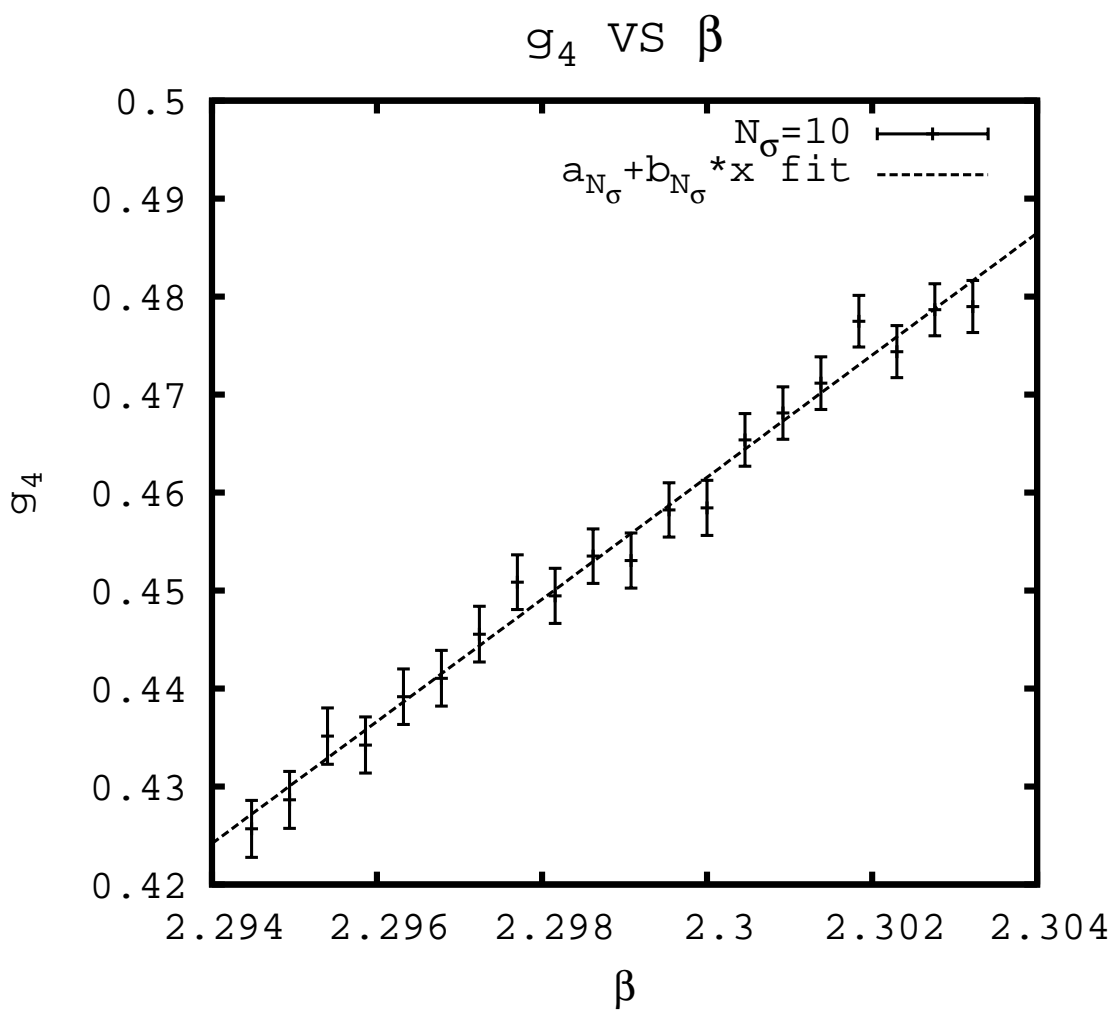

Figure 4.2: Linear fit of $g_{4}$ near $\beta_{c}$ for $N_{\sigma}=10,\left(\beta_{c}\right)_{a p p}=2.299$ and $(1 / \nu)_{a p p}=1.6$. 


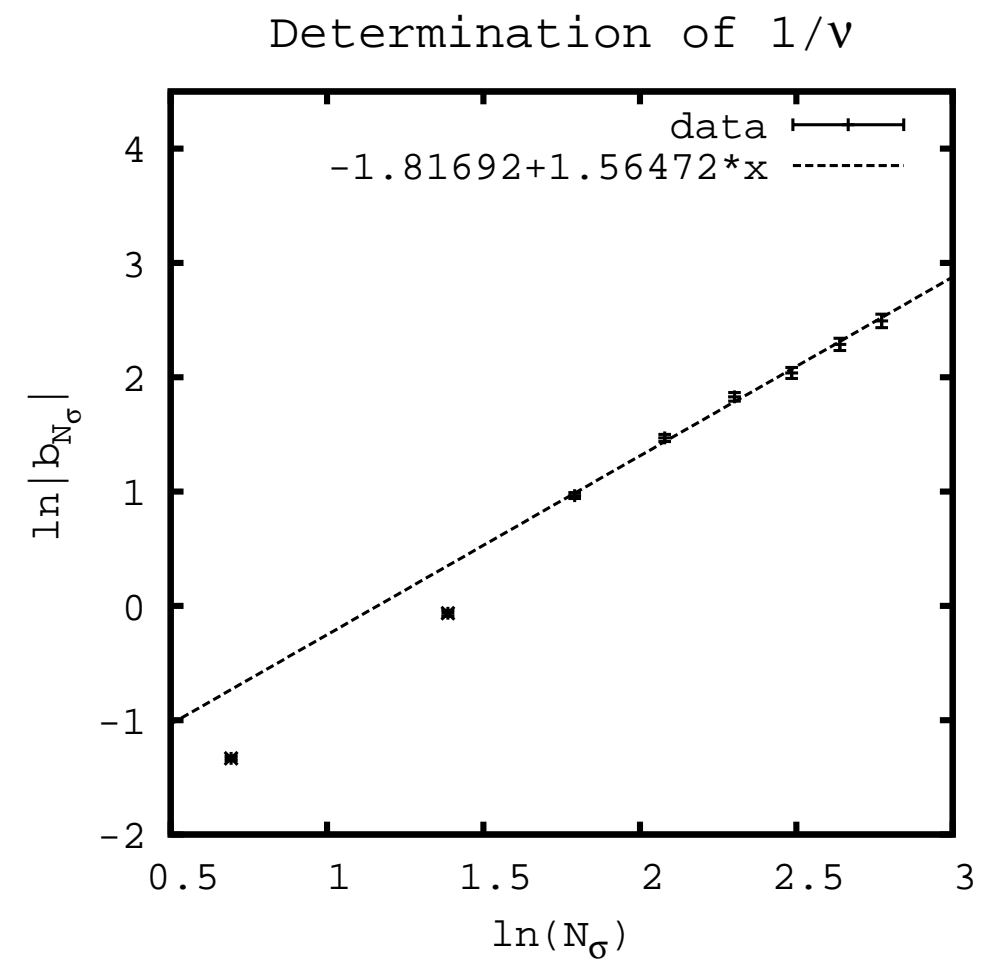

Figure 4.3: Determination of $1 / \nu$ from the $\log -\log$ fit discussed in the text. 
linear fit are

$$
\begin{aligned}
& a_{N_{\sigma}} \simeq g_{4}\left(\beta_{c}, \infty\right)+c_{0} N_{\sigma}^{-\omega}-f_{1} N_{\sigma}^{1 / \nu} \\
& b_{N_{\sigma}} \simeq f_{1} N_{\sigma}^{1 / \nu} / \beta_{c} .
\end{aligned}
$$

Once we have $b_{N_{\sigma}}$ for different $N_{\sigma}$, we can do a log-log fit to determine the inverse critical exponent $1 / \nu$. One should note that the slope can be determined independently of $\beta_{c}$ or $g_{4}\left(\beta_{c}, \infty\right)$. This is illustrated in Fig. 4.3. From the log-log fit with $N_{\sigma} \geq 6$, we obtain $1 / \nu=1.56(4)$. We plan to model and explain the deviations from linearity at low $N_{\sigma}$.

We need to address the dependence on $\left(\beta_{c}\right)_{a p p}$ and $(1 / \nu)_{a p p}$. We used the central value $\beta_{c}=2.2991$ from Ref. [176] as the critical value and changed the center of the interval $\left(\beta_{c}\right)_{a p p}$, between 2.297 and 2.301 and $(1 / \nu)_{a p p}$ between 1.4 and 1.8. We calculated $1 / \nu$ for a set of 189 values of $\left(\left(\beta_{c}\right)_{a p p},(1 / \nu)_{a p p}\right)$. The histogram is shown in Fig. 4.4. We can see that the values appear in a rather large range between 1.468 and 1.631. The average of this set of results is $\overline{1 / \nu}=1.570$ with $\sigma=0.027$ which will be our preliminary result. In the meantime, we collected more data at large $N_{\sigma}$. If we choose the center of the data in a smaller range, namely with $\left(\beta_{c}\right)_{a p p}$ from 2.298 and 2.300 and the same range for $(1 / \nu)_{a p p}$, we get a slightly different distribution shown in Fig. 4.5, but the average is essentially the same. It seems thus possible to average over $\left(\beta_{c}\right)_{a p p}$ and $(1 / \nu)_{a p p}$ in order to get a more accurate value of $1 / \nu$. For reference, $1 / \nu_{\text {Ising }}$ is estimated as 1.5887(85) in Ref. [183] and 1.5878(12) in Ref. [180] and our preliminary result here is consistent with these more accurate values. This is preliminary, we plan to go to larger $N_{\sigma}$ and to determine $\beta_{c}$ independently using the method of Ref. [176]. 


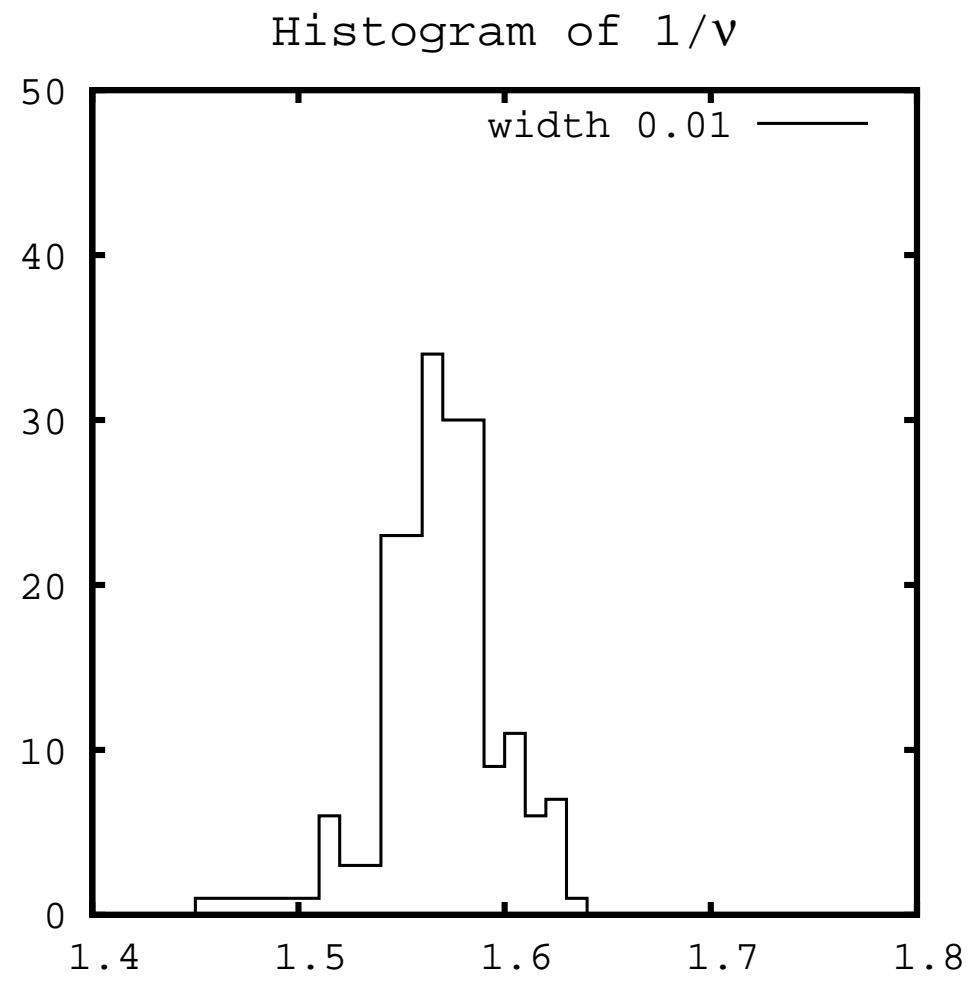

Figure 4.4: $\left(\beta_{c}\right)_{a p p}$ changes from 2.297 to $2.301 ;(1 / \nu)_{a p p}$ changes from 1.4 to 1.8 . $\overline{1 / \nu}=1.570 ; \sigma=0.027$. 


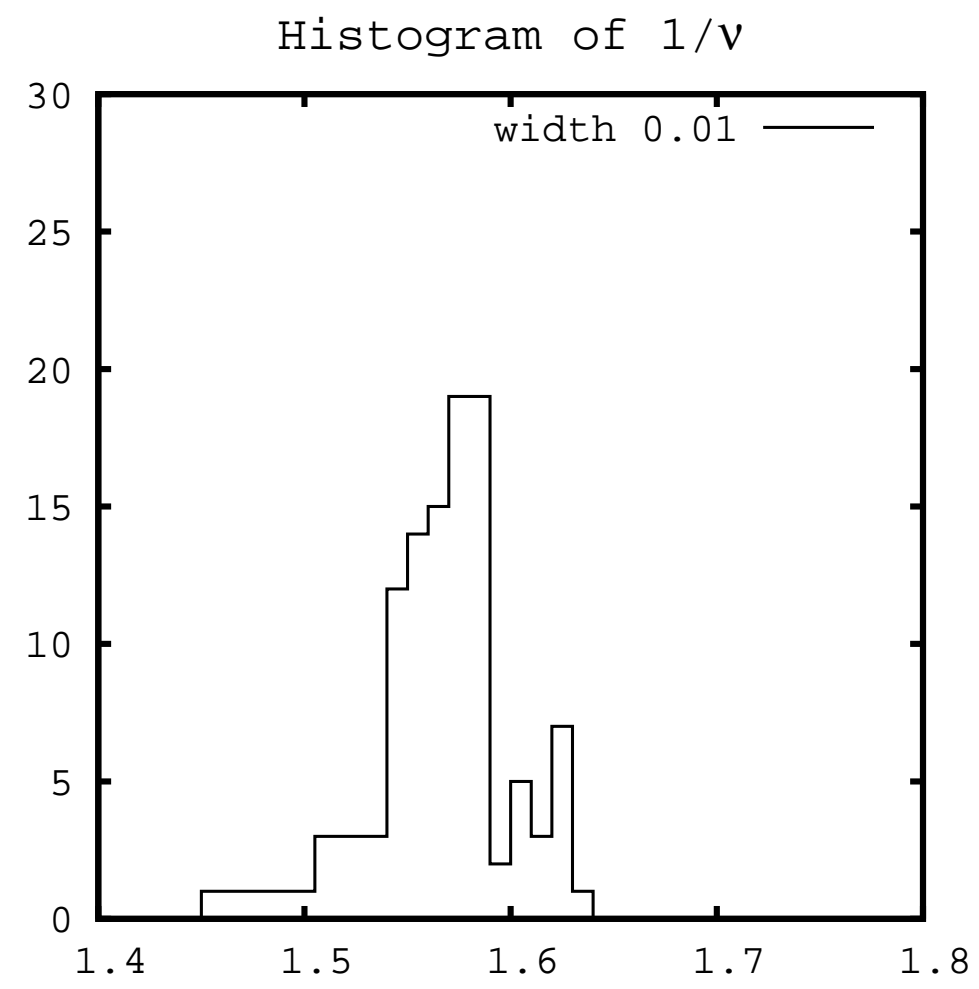

Figure 4.5: $\left(\beta_{c}\right)_{\text {app }}$ changes from 2.298 to 2.300; $(1 / \nu)_{\text {app }}$ changes from 1.4 to 1.8 . $\overline{1 / \nu}=1.571 ; \sigma=0.028$. 


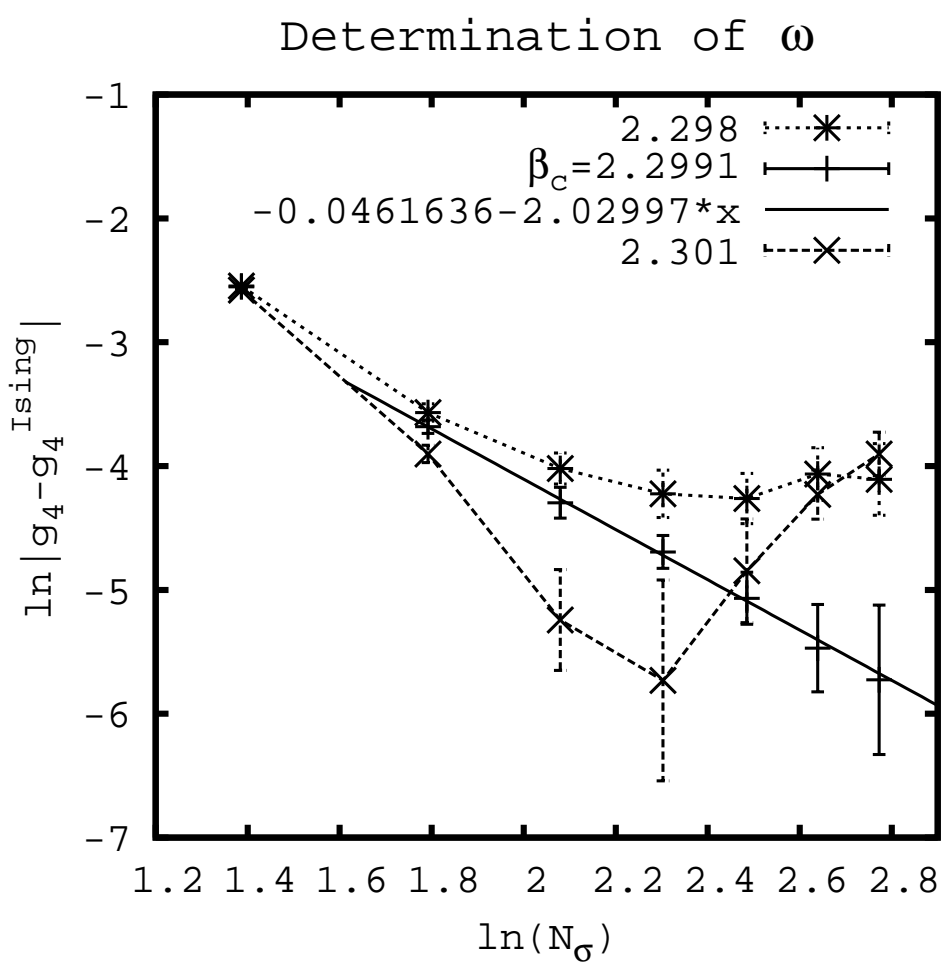

Figure 4.6: For $\beta_{c}=2.2991$, the behavior is approximately linear: $g_{4} \simeq g_{4}\left(\beta_{c}, \infty\right)+$ $c_{0} \times N_{\sigma}^{-\omega}$.

\subsubsection{Determination of the critical exponent $\omega$}

Unless we determine $\beta_{c}$ and $1 / \nu$ very precisely, it is very difficult to subtract the effects of the third term of Eq. (4.4). If we can work at $\beta_{c}$, this term is absent:

$$
g_{4}\left(\beta_{c}, N_{\sigma}\right)=g_{4}\left(\beta_{c}, \infty\right)+c_{0} N_{\sigma}^{-\omega}
$$

Consistently with the previous section and the rest of the literature, we assume the universal value $g_{4}\left(\beta_{c}, \infty\right)=0.46575$ as found in Ref. [180]. $\log \left[\left|g_{4}-g_{4}\left(\beta_{c}, \infty\right)\right|\right]$ vs. $\log \left[N_{\sigma}\right]$ should be linear right at $\beta_{c}$ and nonlinear for all the other $\beta$ s. This is shown in Figure 4.6. At the same time, the slope is $-\omega$. The result we obtained from this analysis is $\omega=2.030(36)$. 
This is very different from $\omega_{\text {Ising }}=0.812$ [183]. It is possible that the coefficient of the $N_{\sigma}^{-\omega}$ is very small and the exponent we extrapolated is a sub-subleading exponent. For a detail discussion of the sub-leading corrections, see Ref. [180]. The most plausible explanation seems that this exponent is related to the irrelevant direction associated with the breaking of rotational symmetry [184] and which is close to 2 .

\subsection{Infrared Fixed Point of the $S U(3)$ Multifla- vor Lattice Gauge Theory}

With the ongoing effort at the LHC, there has been a renewed interest in the phase diagram of lattice gauge theory models. Theories that have an infrared fixed point (IRFP) and are asymptotically free(AF) at high energies are called inside the "conformal window". The location of the conformal windows for several families of models have triggered intense discussions. Different numerical and analytical techniques have been applied to QCD-like models with a large number of fermion flavors or with fermions in higher representations It is important to understand the critical behavior of lattice models from various points of view. It was proposed $[1,123]$ to consider complex extensions of the picture of confinement proposed by Tomboulis. It was observed that the Fisher zeros, the zeros of the partition function in the complex $\beta$ plane, determine the global properties of the complex RG flows. In the case where a phase transition is present, the scaling properties of the zeros allow us to distinguish between a first and second order phase transition. In the following, we briefly review the Finite Size Scaling (FSS) of the Fisher zeros. We then discuss numerical results for $S U(3)$ in $4 \mathrm{D} N_{f}=4$ and 12 flavors.

The Fisher zeros for $S U(3)$ with $N_{f}=4$ and 12 quarks in the fundamental representation have been investigated. We used the standard Wilson gauge action and unimproved staggered fermion action with the Rational Hybrid Monte Carlo 
(RHMC) algorithm $[185,186,187]$. We started with relatively small symmetric lattices and up to 50,000 configurations. The bare quark mass is set to be $m_{q}=0.02$ for now. For $N_{f}=12$, we found a discontinuity for the plaquette near $\beta \simeq 4.1$ for $V=8^{4}$ lattices. The plaquette histories showed the characteristic hysteresis behavior. For comparison, we show the crossover for $N_{f}=4$. Possible hypothesis to be tested are that for $N_{f}=12$, the imaginary part scales like $L^{-4}$, which signals a first order phase transition and that the real part increases like $\log (L)$. Nonlinear effects seem important at small volume and larger volume calculations are in progress. The chiral condensate has a discontinuity near the same value of $\beta$ as the plaquette. More recently, it was shown that improved actions can create a second discontinuity with a broken single-site shift symmetry between the two transitions.

We know that RG flows are defined in the space of couplings: $\left(g_{1}, g_{2}, g_{3}, \cdots\right)$. $\beta_{C S}$ functions describe how the couplings change with energy scales $\Lambda$ :

$$
\beta_{C S}\left(g_{i}\right)=\Lambda \frac{\partial}{\partial \Lambda} g_{i}
$$

In general, the beta function, $\beta(g)$, depends on the renormalization scheme. However, the first two terms are scheme independent under the perturbative expansion. The two loop beta function can be calculated for arbitrary $N_{c}$ and $N_{f}$

$$
\begin{gathered}
\beta(a)=-\beta_{0} a^{2}-\beta_{1} a^{3}+\cdots, \\
\beta_{0}=\frac{11}{3} N_{c}-\frac{2}{3} N_{f}, \\
\beta_{1}=\frac{34 N_{c}^{2}}{3}-\frac{10 N_{c} N_{f}}{3}-\frac{\left(-1+N_{c}^{2}\right) N_{f}}{N_{c}},
\end{gathered}
$$

where $a \equiv \frac{g^{2}}{4 \pi^{2}}=\frac{\alpha}{4 \pi}$ The UV and IR properties of the theory depend on the signs of the coefficients. This is illustrated in Fig. 4.7, 4.8, and 4.9.

The partition function for full QCD with one fermion flavor is

$$
Z=\int[d U][d \bar{\psi}][d \psi] \exp \left[-S_{G}(U)-\bar{\psi} M(U) \psi\right], \quad M=D+m .
$$



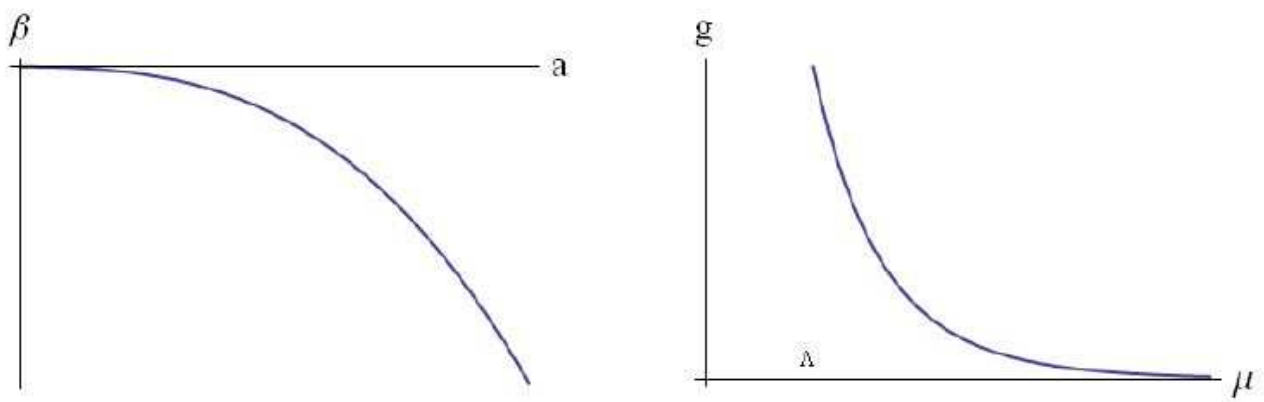

Figure 4.7: $\beta_{0}>0$ and $\beta_{1}>0$.
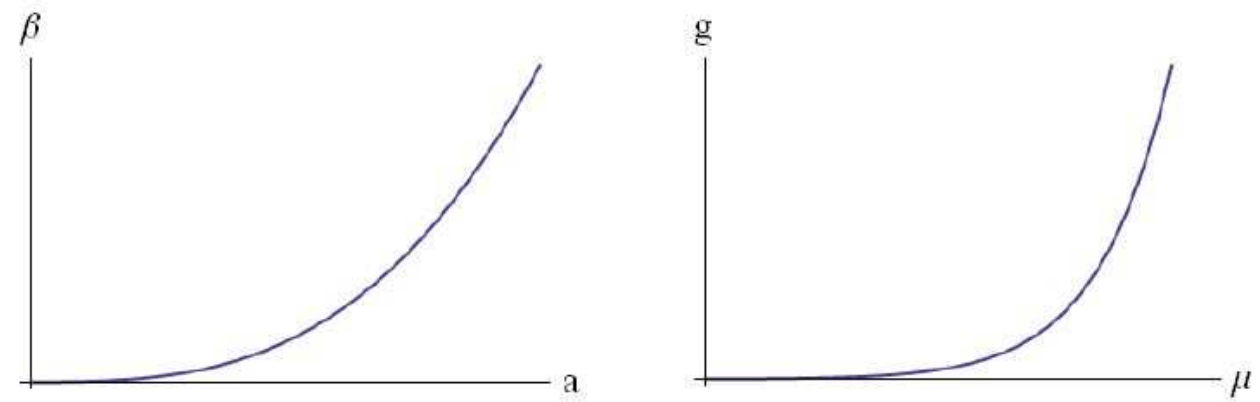

Figure 4.8: $\beta_{0}<0$ and $\beta_{1}<0$.
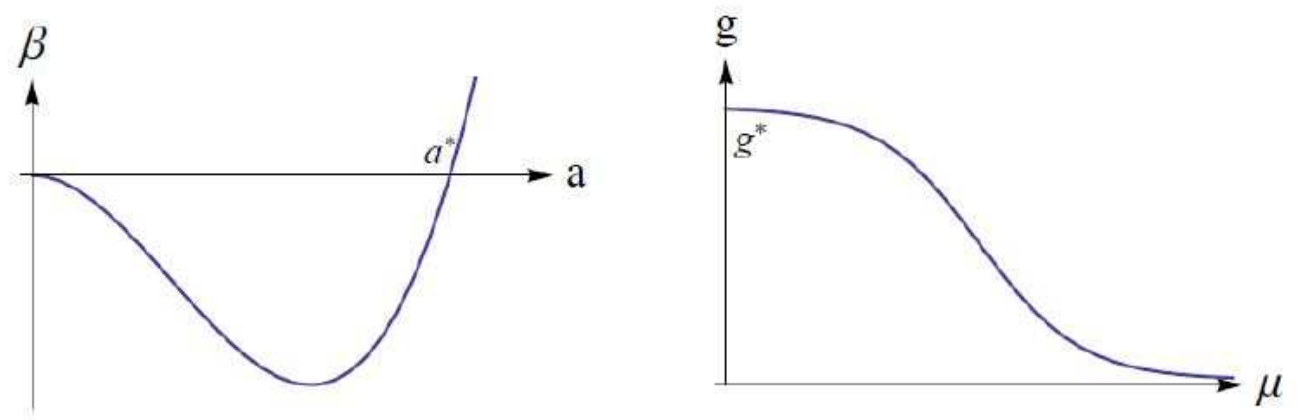

Figure 4.9: $\beta_{0}>0$ and $\beta_{1}<0$. 


\begin{tabular}{|r|r|r|r|}
\hline$N_{f}$ & Volume & Num. of $\beta$ used & Num. of config. per $\beta$ \\
\hline 4 & $4^{4}$ & 21 & 25,000 \\
\hline 4 & $6^{4}$ & 35 & 8,000 \\
\hline 4 & $8^{4}$ & 36 & 25,000 \\
\hline 4 & $12^{4}$ & 21 & 25,000 \\
\hline 4 & $16^{4}$ & 5 & 2,500 \\
\hline 12 & $4^{4}$ & 31 & 50,000 \\
\hline 12 & $6^{4}$ & 41 & 50,000 \\
\hline 12 & $8^{4}$ & 15 & 8,000 \\
\hline
\end{tabular}

Integrating over Grassmann variables

$$
Z=\int[d U] \exp \left[-S_{G}(U)\right] \operatorname{det} M(U)
$$

Introducing a pseudo fermion field $\Phi$

$$
\left.Z=\int[d U]\left[d \Phi^{*}\right][d \Phi] \exp \left[-S_{G}(U)\right]-\Phi^{*} M(U) \Phi\right] .
$$

For 2 flavors, one would have

$$
Z=\int[d U] \exp \left[-S_{G}(U)\right] \operatorname{det}\left[M^{\dagger} M\right], \quad \operatorname{det} D=\operatorname{det} D^{\dagger} .
$$

For staggered fermion

$$
Z=\int[d U] \exp \left[-S_{G}(U)\right] \prod_{f} \operatorname{det}\left[M^{\dagger} M\right]^{N_{f} / 8} .
$$

We use standard Wilson gauge and naive staggered fermion action with the Hybrid Monte Carlo (HMC) algorithm. Focus on $N_{f}=4$ and $N_{f}=12$ with relatively small symmetric lattices. Bare quark mass is set to be $m_{q}=0.02$ for now. The following is the configurations used to calculate Fisher zeros 


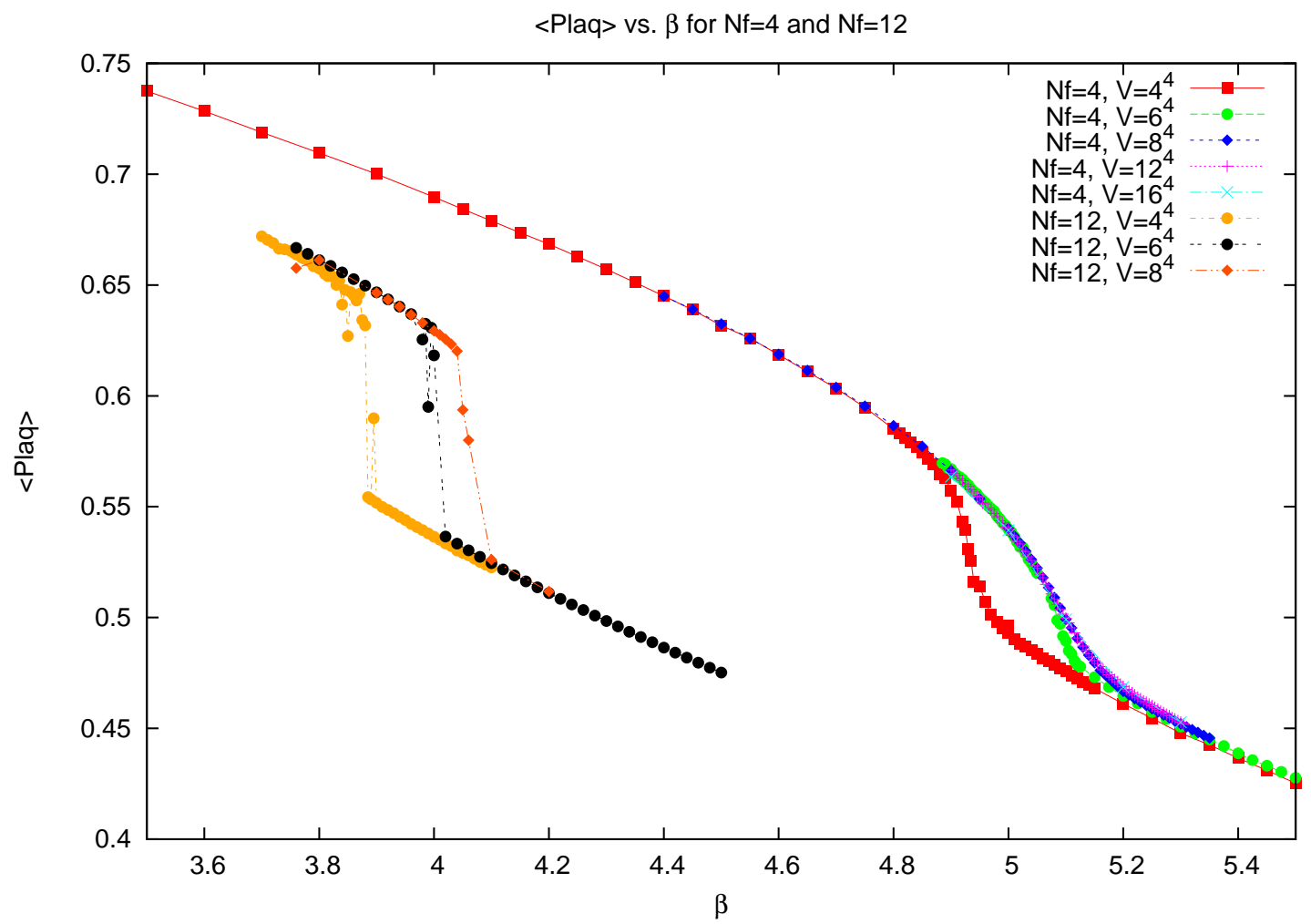

Figure 4.10: Average plaquette for $N_{f}=4$ and $N_{f}=12$ at different volumes. 


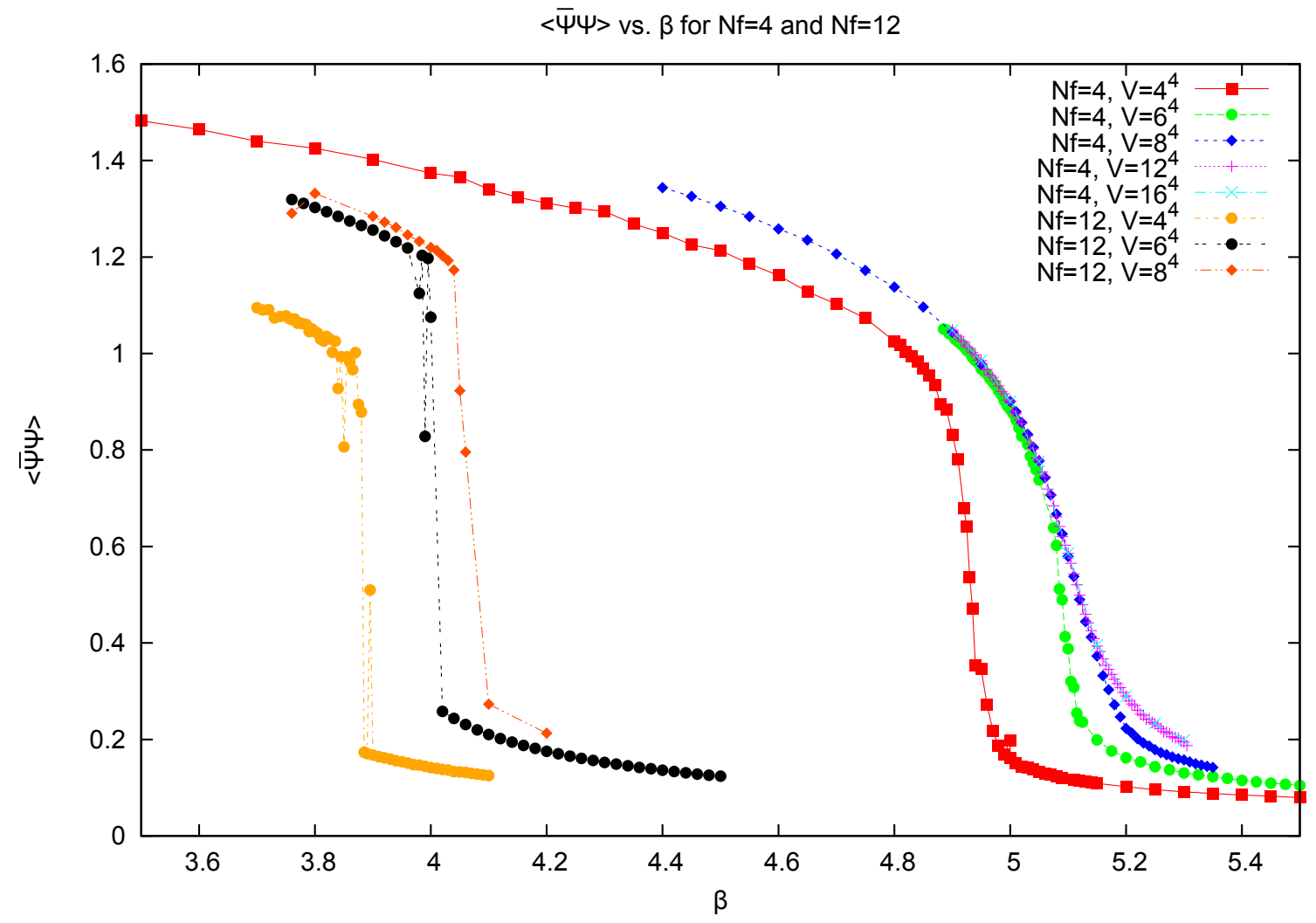

Figure 4.11: $\left\langle\bar{\Psi} \Psi>\right.$ for $N_{f}=4$ and $N_{f}=12$ at different volumes. 
Fisher's zeros for $\mathrm{Nf}=4$ and $\mathrm{Nf}=12, \mathrm{~m}=0.02$

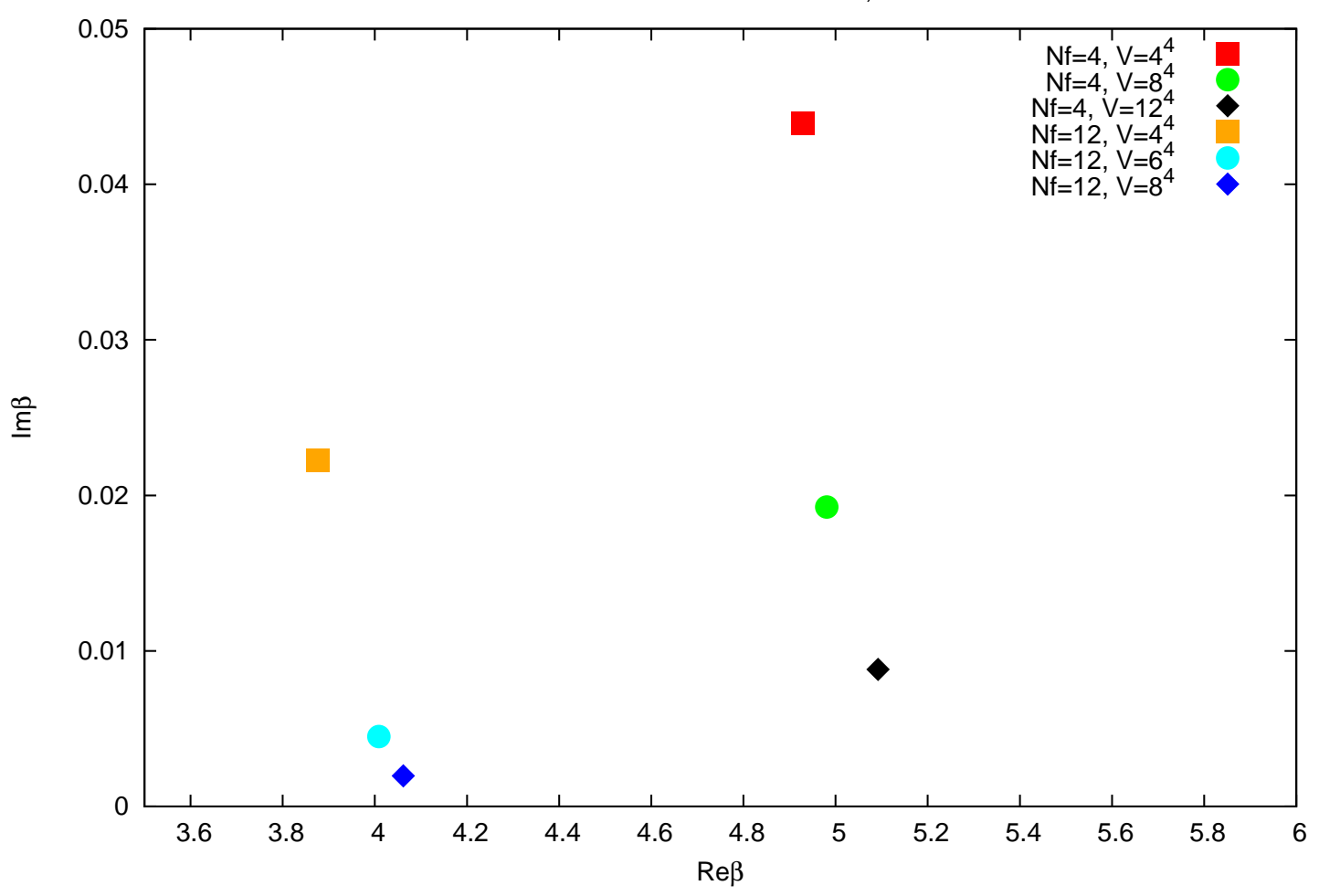

Figure 4.12: Fisher zeros for $N_{f}=4$ and $N_{f}=12$ at different volumes. 


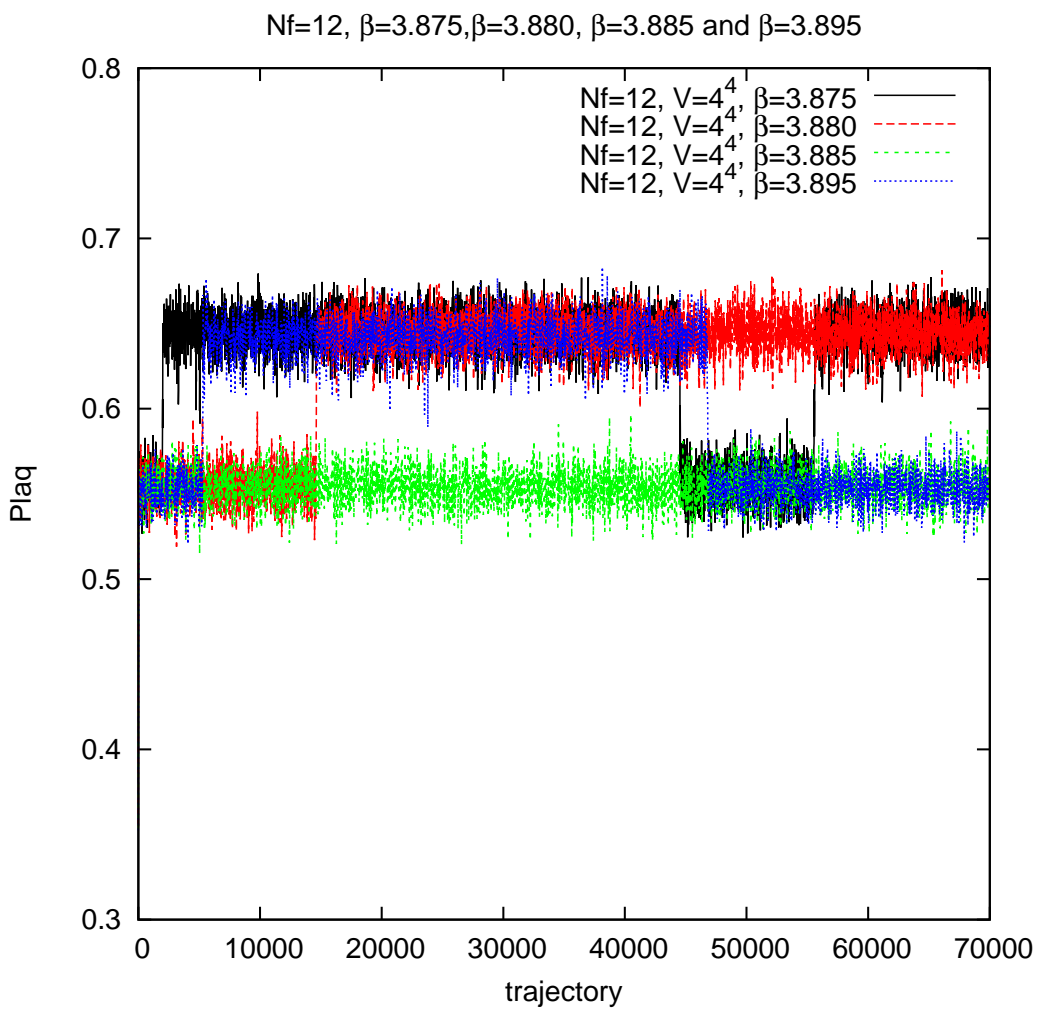

Figure 4.13: Typical plaquette history for the $\mathrm{V}=4^{4}$ system. 


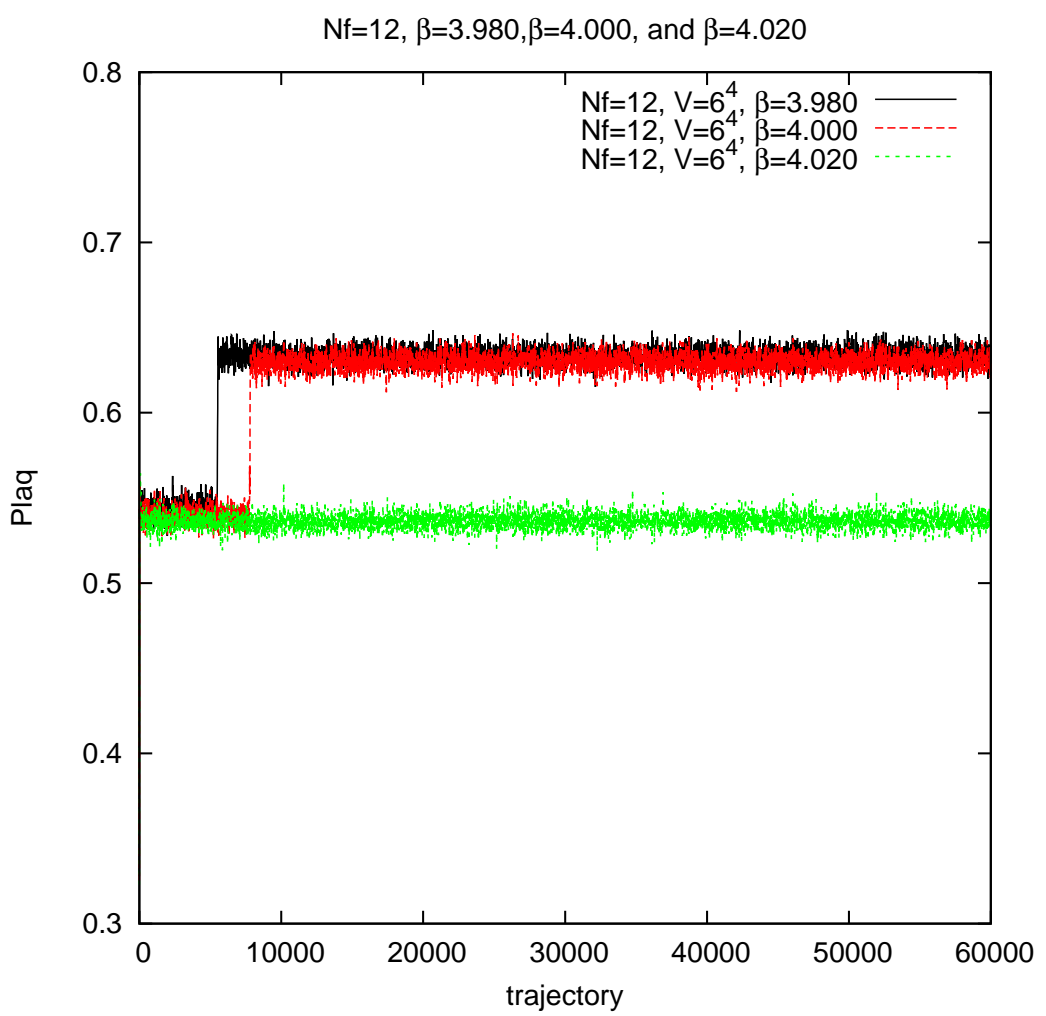

Figure 4.14: Typical plaquette history for the $\mathrm{V}=6^{4}$ system. 


\section{CHAPTER 5 TENSOR RENORMALIZATION GROUP}

\subsection{TRG on 2D $O(2)$ model}

The Hamiltonian for the two dimensional $O(2)$ model can be written as

$$
H=-\sum_{<i j>} \mathbf{S}_{\mathbf{i}} \cdot \mathbf{S}_{\mathbf{j}}
$$

where $\mathbf{S}_{\mathbf{i}}$ is the unit vector staying at each site $i:(\cos (\theta), \sin (\theta))$.

$H$ then can be written as

$$
H=-\sum_{<i j>} \cos \left(\theta_{i}-\theta_{j}\right) \quad\left(\pi<\theta_{i}<\pi\right) .
$$

The corresponding partition function is

$$
Z=\int \prod_{i} \frac{d \theta_{i}}{2 \pi} e^{\beta \sum_{i j>} \cos \left(\theta_{i}-\theta_{j}\right)} .
$$

We can further expand the $e^{\beta \cos \theta}$ term as sum of modified Bessel function of the first kind

$$
\begin{aligned}
e^{\beta \cos \theta} & =I_{0}(\beta)+\sum_{n=1}^{\infty} I_{n}(\beta)\left(e^{i n \theta}+e^{-i n \theta}\right) \\
& =I_{0}(\beta)\left[1+\sum_{n=-\infty, n \neq 0}^{\infty} \frac{I_{n}(\beta)}{I_{0}(\beta)} e^{i n \theta}\right] \\
& =I_{0}(\beta) \sum_{n=-\infty}^{+\infty} \frac{I_{n}(\beta)}{I_{0}(\beta)} e^{i n \theta}
\end{aligned}
$$

Modified Bessel functions listed above have the following asymptotic properties

$$
\frac{I_{n}(\beta)}{I_{0}(\beta)}=\frac{I_{-n}(\beta)}{I_{0}(\beta)} \sim \begin{cases}\frac{\beta^{n}}{2^{n} n !} & \beta \rightarrow 0 \\ e^{-\frac{n^{2}}{2 \beta}} & \beta \rightarrow \infty .\end{cases}
$$

By using Eq. (5.7), one could perform conventional high temperature expansion and so on. However, in the following, we would like to write down the partition function as product of matrices 


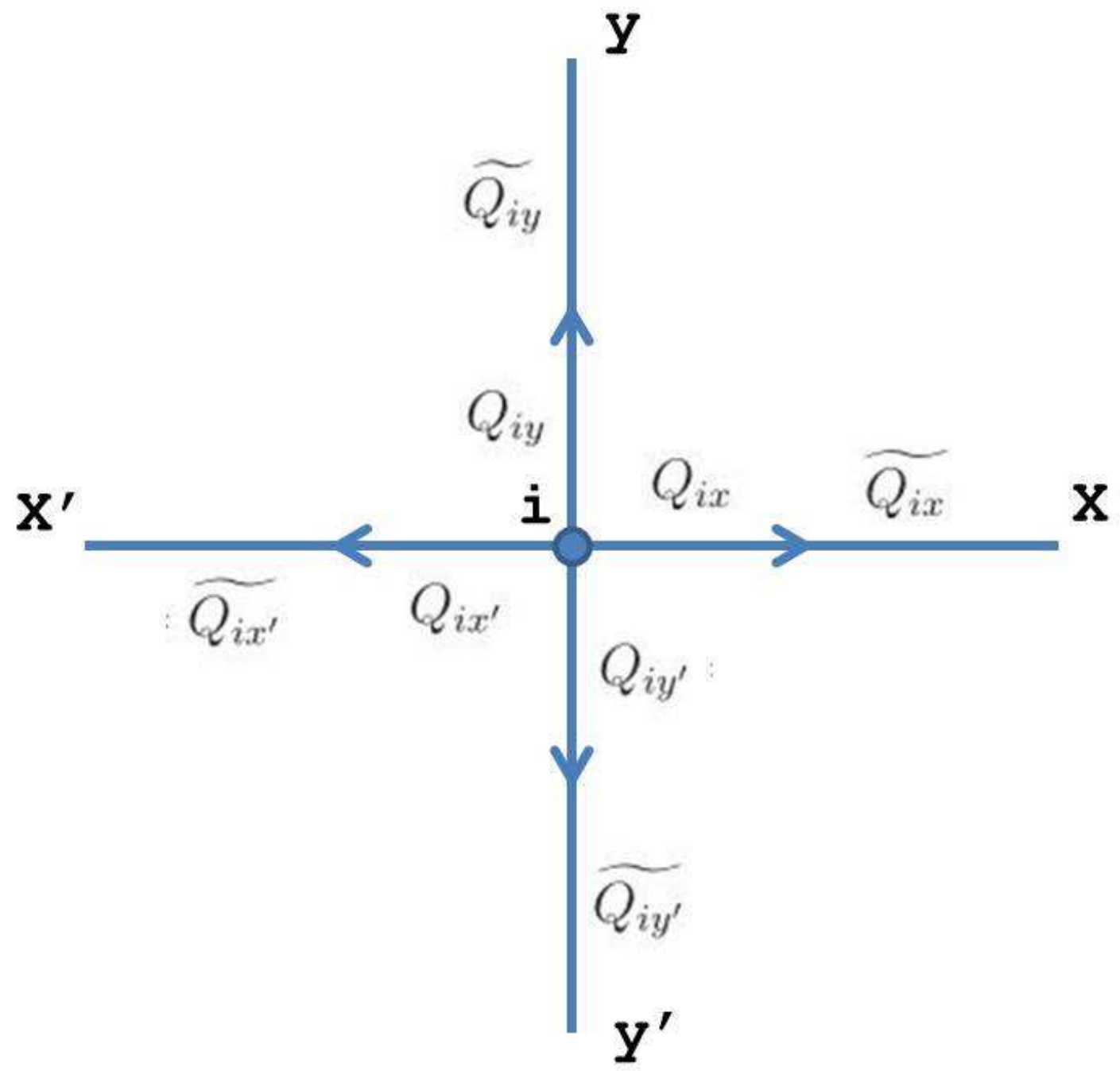

Figure 5.1: Tensor representation of the two dimensional $O(2)$ model. 


$$
\begin{aligned}
Z(\beta) & =\int \prod_{k} \frac{d \theta_{k}}{2 \pi} \prod_{<i j>} e^{\beta \cos \left(\theta_{i}-\theta_{j}\right)} \\
& =\int \prod_{k} \frac{d \theta_{k}}{2 \pi} \prod_{<i j>n_{i j}=-\infty} \sum_{n_{i j}}^{+\infty}(\beta) e^{i n_{i j}\left(\theta_{i}-\theta_{j}\right)} \\
& =\int \prod_{k} \frac{d \theta_{k}}{2 \pi} \prod_{<i j>} W\left(\theta_{i}, \theta_{j}\right) .
\end{aligned}
$$

where $W\left(\theta_{i}, \theta_{j}\right)=e^{\beta \cos \left(\theta_{i}-\theta_{j}\right)}=\sum_{n_{i j}=-\infty}^{+\infty} e^{i n_{i j}\left(\theta_{i}-\theta_{j}\right)} I_{n_{i j}}(\beta)$.

$W\left(\theta_{i}, \theta_{j}\right)$ can be further written as

$$
W\left(\theta_{i}, \theta_{j}\right)=\sum_{n_{i j}} U\left(\theta_{j}, n_{i j}\right) \Lambda_{n_{i j}} V\left(\theta_{i}, n_{i j}\right)
$$

where

$$
\begin{gathered}
U\left(\theta_{j}, n_{i j}\right)=e^{-i n_{i j} \theta_{j}}, \\
\Lambda_{n_{i j}}=I_{n_{i j}}(\beta), \\
V\left(\theta_{i}, n_{i j}\right)=e^{+i n_{i j} \theta_{i}} .
\end{gathered}
$$

We define

$$
\begin{aligned}
& Q_{i j}=\sqrt{I_{n_{i j}}(\beta)} e^{i n_{i j} \theta_{i}} \\
& \widetilde{Q_{i j}}=\sqrt{I_{n_{i j}}(\beta)} e^{-i n_{i j} \theta_{j}},
\end{aligned}
$$

and write $W\left(\theta_{i}, \theta_{j}\right)$ as

$$
W\left(\theta_{i}, \theta_{j}\right)=\sum_{n_{i j}} Q_{i j} \widetilde{Q_{i j}}
$$

We can then assign each link with a summation over product of $Q$ and $\widetilde{Q}$, see Fig.

\section{1 for example.}

The tensor $T$ at site $i$ can be defined as product of all the $Q$ and $\widetilde{Q}$ that connect to $i$ and integrate over the variable $\theta_{i}$. Since $\frac{1}{2 \pi} \int d \theta e^{i \theta\left(n_{1}-n_{2}\right)}=\delta\left(n_{1}-n_{2}\right)$, we will get element of tensor $T$ as sum of delta function times modified Bessel function. In principle, we have infinite terms in the summation. In practice, we choose to keep 
only the first several terms with the constraint $\left|n_{i j}\right| \leq n_{\max }$. The element of $T$ is

$$
\begin{aligned}
T_{x x^{\prime} y y^{\prime}}^{i}\left(n_{i x}, n_{i y}, n_{i x^{\prime}}, n_{i y^{\prime}}\right)= & \sqrt{I_{n_{i x}}(\beta)} \sqrt{I_{n_{i y}}(\beta)} \sqrt{I_{n_{i x^{\prime}}}(\beta)} \sqrt{I_{n_{i y^{\prime}}}(\beta)} \\
& * \delta\left(n_{i x}+n_{i y}+n_{i x^{\prime}}+n_{i y^{\prime}}\right) .
\end{aligned}
$$

We can apply similar trick to higher dimensions.

\subsection{TRG on $2 \mathrm{D} \mathrm{O}(3)$ model}

The Hamiltonian for the two dimensional $O(3)$ model is defined as

$$
H=-\sum_{<i j>} \mathbf{S}_{\mathbf{i}} \cdot \mathbf{S}_{\mathbf{j}}
$$

where $\mathbf{S}_{\mathbf{i}}$ and $\mathbf{S}_{\mathbf{j}}$ are unit vectors staying at each site $i:(\sin \theta \cos \phi, \sin \theta \sin \phi, \cos \theta)$ and the summation is taken over all the nearest neighbor pairs of spins in the lattice.

H can then be written as

$$
H=-\sum_{<i j>} \cos \gamma_{i j}
$$

where $\gamma_{i j}$ is the angle between $\mathbf{S}_{\mathbf{i}}$ and $\mathbf{S}_{\mathbf{j}}$ and $\cos \gamma_{i j}$ can be expressed in terms of $\theta$ and $\phi$

$$
\cos \gamma_{i j}=\cos \theta_{i} \cos \theta_{j}+\sin \theta_{i} \sin \theta_{j} \cos \left(\phi_{i}-\phi_{j}\right) .
$$

The partition function can be written as

$$
\begin{aligned}
Z_{N} & =\int \prod_{k=1}^{N} \frac{d \Omega_{k}}{4 \pi} e^{-\beta H} \\
& =\int \prod_{k=1}^{N} \frac{d \Omega_{k}}{4 \pi} e^{\beta \sum_{<i j>} \cos \gamma_{i j}} \\
& =\int \prod_{k=1}^{N} \frac{d \Omega_{k}}{4 \pi} \prod_{<i j>} e^{\beta \cos \gamma_{i j}} .
\end{aligned}
$$

We can further expand $e^{\beta \cos \gamma}$ in terms of modified Bessel function of the first kind 
as

$$
\begin{aligned}
e^{\beta \cos \gamma} & =I_{0}(\beta)+\sum_{n=1}^{\infty} I_{n}(\beta)\left(e^{i n \gamma}+e^{-i n \gamma}\right) \\
& =I_{0}(\beta)+2 \sum_{n=1}^{\infty} I_{n}(\beta) \cos (n \gamma) .
\end{aligned}
$$

In the following, three more special functions will be involved: Chebyshev polynomials of the first kind $T_{n}(x)$; Legendre polynomials $P_{n}(x)$; and spherical harmonic $Y_{l m}(\theta, \phi)$.

By definition, Chebyshev polynomials of the first kind satisfy

$$
T_{n}(\cos \gamma)=\cos (n \gamma)
$$

and the right hand side of the above equation Eq. 5.23 can be written as a sum of the Legendre polynomials $P_{n}(\cos \gamma)$

$$
\begin{aligned}
T_{n}(\cos \gamma) & =\cos (n \gamma) \\
& =\sum_{l=0}^{n} a_{n l} P_{l}(\cos \gamma) .
\end{aligned}
$$

The coefficient $a_{n l}$ can be obtained by using the orthogonality property of the Legendre polynomials

Therefore,

$$
\int_{-1}^{1} P_{m}(x) P_{n}(x) d x=\frac{2}{2 m+1} \delta_{m n} .
$$

$$
a_{n l}=\frac{2 l+1}{2} \int_{-1}^{1} T_{n}(x) P_{l}(x) d x .
$$

Combining Eq. (5.22) and Eq. (5.24), one would get

$$
e^{\beta \cos \gamma_{i j}}=I_{0}(\beta)+2 \sum_{n=1}^{\infty} I_{n}(\beta) \sum_{l=0}^{n} a_{n l} P_{l}\left(\cos \gamma_{i j}\right) .
$$

By using the following addition theorem for spherical harmonic

$$
P_{l}\left(\cos \gamma_{i j}\right)=\frac{4 \pi}{2 l+1} \sum_{m=-l}^{l} Y_{l m}^{*}\left(\theta_{j}, \phi_{j}\right) Y_{l m}\left(\theta_{i}, \phi_{i}\right) \text {. }
$$


Eq. (5.27) can be written as

$$
e^{\beta \cos \gamma_{i j}}=I_{0}(\beta)+2 \sum_{n=1}^{\infty} I_{n}(\beta) \sum_{l=0}^{n} a_{n l} \frac{4 \pi}{2 l+1} \sum_{m=-l}^{l} Y_{l m}^{*}\left(\theta_{j}, \phi_{j}\right) Y_{l m}\left(\theta_{i}, \phi_{i}\right) .
$$

Finally, we get the expression of $e^{\beta \cos \gamma_{i j}}$ as functions of spherical harmonics

$$
e^{\beta \cos \gamma_{i j}}=\sum_{l=0}^{\infty} A_{l}(\beta) \sum_{m=-l}^{l} Y_{l m}^{*}\left(\theta_{j}, \phi_{j}\right) Y_{l m}\left(\theta_{i}, \phi_{i}\right),
$$

where

$$
\begin{aligned}
A_{0}(\beta) & =I_{0}(\beta)+2 \sum_{n=1}^{\infty} I_{n}(\beta) a_{n 0} 4 \pi \\
A_{1}(\beta) & =2 \sum_{n=1}^{\infty} I_{n}(\beta) a_{n 1} \frac{4 \pi}{3} \\
\vdots & \\
A_{l}(\beta) & =2 \sum_{n=l}^{\infty} I_{n}(\beta) a_{n l} \frac{4 \pi}{2 l+1} .
\end{aligned}
$$

The element of the tensor $T$ can then be written as

$$
\begin{aligned}
& T_{\left(l_{1}, m_{1}\right),\left(l_{2}, m_{2}\right),\left(l_{3}, m_{3}\right),\left(l_{4}, m_{4}\right)}= \\
& \int_{0}^{2 \pi} d \phi \int_{0}^{\pi} d \theta \sin \theta Y_{l_{1} m_{1}}(\theta, \phi) Y_{l_{2} m_{2}}(\theta, \phi) \\
& Y_{l_{3} m_{3}}^{*}(\theta, \phi) Y_{l_{4} m_{4}}^{*}(\theta, \phi) \sqrt{A_{l_{1}} A_{l_{2}} A_{l_{3}} A_{l_{4}}} .
\end{aligned}
$$

The direction convention is shown in Figure 5.2. Equation (5.32) can be further simplified by expanding the product of two spherical harmonics in terms of spherical harmonics themselves

$$
Y_{l_{1} m_{1}}(\theta, \phi) Y_{l_{2} m_{2}}(\theta, \phi)=\sum_{L=l_{\min }}^{l_{\max }} G_{L}^{\left(m_{1}, m_{2}, l_{1}, l_{2}\right)} Y_{L}^{m_{1}+m_{2}}(\theta, \phi),
$$

where $G_{L}^{\left(m_{1}, m_{2}, l_{1}, l_{2}\right)}$ is Gaunt coefficients. Different methods have been developed to effectively calculate these coefficients. The summation bounds are given in the 
following

$$
\begin{aligned}
& l_{\text {max }}=l_{1}+l_{2} \\
& l_{\text {min }}=\left\{\begin{array}{lll}
\lambda_{\text {min }} & \text { if } & l_{\text {max }}+\lambda_{\text {min }} \text { is even } \\
\lambda_{\text {min }}+1 & \text { if } & l_{\text {max }}+\lambda_{\text {min }} \text { is odd }
\end{array}\right. \\
& \lambda_{\text {min }}=\max \left(\left|l_{1}-l_{2}\right|,\left|m_{1}+m_{2}\right|\right) .
\end{aligned}
$$

The angular integration in Eq. (5.32) can be performed in the following

$$
\begin{aligned}
& \int_{0}^{2 \pi} d \phi \int_{0}^{\pi} d \theta \sin \theta Y_{l_{1} m_{1}}(\theta, \phi) Y_{l_{2} m_{2}}(\theta, \phi) Y_{l_{3} m_{3}}^{*}(\theta, \phi) Y_{l_{4} m_{4}}^{*}(\theta, \phi) \\
& =\int_{0}^{2 \pi} d \phi \int_{0}^{\pi} d \theta \sin \theta \sum_{L=l_{\min }}^{l_{\max }} G_{L}^{\left(m_{1}, m_{2}, l_{1}, l_{2}\right)} Y_{L}^{m_{1}+m_{2}}(\theta, \phi) \\
& \sum_{L^{\prime}=l^{\prime} \text { min }}^{l^{\prime} \max } G_{L^{\prime}}^{*}{ }^{\left(m_{3}, m_{4}, l_{3}, l_{4}\right)} Y_{L^{\prime}}^{* m_{3}+m_{4}}(\theta, \phi) .
\end{aligned}
$$

By using the orthonormal property of the spherical harmonics

$$
\int_{0}^{2 \pi} d \phi \int_{0}^{\pi} d \theta \sin \theta Y_{l_{1}, m_{1}} Y_{l_{2}, m_{2}}^{*}=\delta_{l_{1} l_{2}} \delta_{m_{1} m_{2}}
$$

along with Equation (5.36), the element of the tensor T (Eq. (5.32)) can then be simplified as

$$
\begin{aligned}
& \left.T_{(} l_{1}, m_{1}, l_{2}, m_{2}, l_{3}, m_{3}, l_{4}, m_{4}\right) \\
& =\delta_{m_{1}+m_{2}, m_{3}+m_{4}} \sum_{L, L^{\prime}} \delta_{L L^{\prime}} G_{L}^{\left(m_{1}, m_{2}, l_{1}, l_{2}\right)} G_{L^{\prime}}^{*}\left(m_{3}, m_{4}, l_{3}, l_{4}\right) \sqrt{A_{l_{1}} A_{l_{2}} A_{l_{3}} A_{l_{4}}} .
\end{aligned}
$$

To summarize, the expression of the element of the tensor T is Eq. (5.38). $G_{L}^{\left(m_{1}, m_{2}, l_{1}, l_{2}\right)}$ and $G_{L^{\prime}}^{*}\left(m_{3}, m_{4}, l_{3}, l_{4}\right)$ are Gaunt coefficients and can be calculated numerically. The summation bounds are given in Eq. (5.34). $A_{l_{1}}, A_{l_{2}}, A_{l_{3}}$, and $A_{l_{4}}$ are listed in Eq. (5.31), where the coefficients $a_{n l}$ can be obtained from Eq. (5.26). 


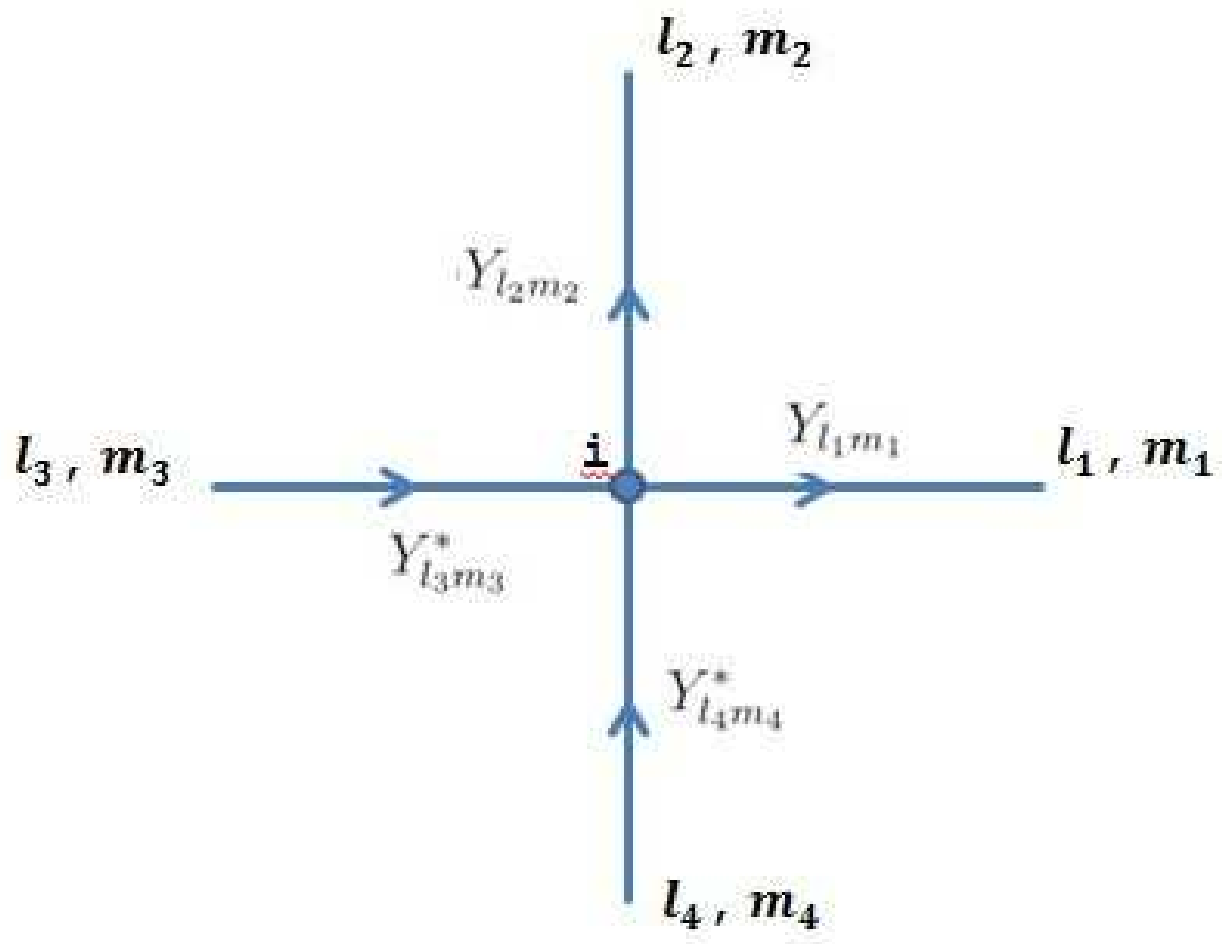

Figure 5.2: Tensor representation of the two dimensional $O(3)$ model. 


\section{CHAPTER 6 CONCLUSION}

In previous chapters, we have discussed several different versions of the renormalization group $(\mathrm{RG})$ methods. These methods have been first explored in spin models and some of them were then used in lattice gauge models.

In Chapter 2, we started with the conventional Ising model and then extensively discussed Dyson's hierarchical model and its variants. The partition function zeros have been calculated and finite size scaling technique has also been applied to get the critical exponents. We also applied the two lattice matching method to construct the RG flows in the complex temperature plane. More importantly, we found out that Fisher zeros, which are partition function zeros in the complex temperature plane, act as a gate controlling the flows. We further discussed the possibility of extending the RG method from discrete block-spinning procedure to the continuous one. We did not succeed here but discussed the obstacle to doing so and tried to quantitatively analyze them.

Inspired by the continuous block-spinning procedure, in Chapter 3, we studied the exact renormalization group (ERG) flow equations and the Wilson-Polchinski equation. We showed that the Wilson-Polchinski equation is equivalent to the ERG equation under the local potential approximation. We also systematically analyzed the cutoff function dependence of the critical exponents. The aim was to find the relation between the ERG equation and the hierarchical approximation and further make the connection between the discrete and continuous transformation.

In Chapter 4, we applied methods developed on spin models to lattice gauge models. We first applied the finite size scaling (FSS) technique to the pure gauge $S U(2)$ lattice gauge model and extrapolated the critical exponents $\nu$ and $\omega$. We then analyzed Fisher zeros of the $S U(3)$ lattice gauge theory with many light flavors. This 
is an on-going project and we are planning on working on it in a more systematic way.

In Chapter 5, we started to formulate the tensor renormalization group on the continuous spin models, $O(2)$ and $O(3)$ (or XY model) in two dimension. We have extended the method to higher dimensions lattice gauge models but I have not included these in the thesis. The advantages of this method are that they can be used to calculate thermodynamic quantities in arbitrary large system, and the systematical uncertainty can be improved systematically.

In summary, RG is an important tool in dealing with many degrees of freedoms, which includes lattice spin and gauge models. Combining with FSS and other numerical techniques, RG becomes an extremely powerful tool in dealing with many interesting but difficult problems in physics. An especially interesting prospect would be to study the conformal and near conformal lattice gauge theories. In those theories, there is more than one scaling variable due to the non-vanishing quark masses. Calculating the running of the coupling and the anomalous dimension associated with the running of the quark masses for those theories will be exciting projects for me in the next couple of years. 


\section{APPENDIX A CRITICAL VALUES FOR THE HIERARCHICAL MODEL}

In order to reliable get the critical exponents, such as $\nu$ and $\omega$, the first step is to locate the critical coupling $\left(\beta_{c}\right)$. It has been shown that the accuracy of $\gamma$ depends on the accuracy of $\beta_{c}$ and the dependence can be approximated by $\delta \gamma \approx\left|\beta-\beta_{c}\right|^{\frac{1}{1+\frac{1}{\Delta}}}=10^{-S D}$, where SD is the accuracy goal for $\gamma$ and $\Delta$ is defined as $\Delta=-\log \left[\lambda_{2}\right] / \log \left[\lambda_{1}\right]$. Table A.1 listed the calculated $\Delta$ for various $l^{D}$. From Table A.1, we can see that $1+1 / \Delta$ is between 3.3 and 3.4. That means we need to calculate $\beta$ up to $10^{-34}$ in order to get an accurate $\gamma$ up to $10^{-10}(S D=10)$.

In our calculation, we always calculate $\beta$ up to $10^{-40}$ or more. Theoretically, we can calculate $\beta$ up to any order. There should be no computer memory problem for this part of calculation.

\begin{tabular}{ccccc}
\hline \hline$l^{D}$ & $\lambda_{1}$ & $\lambda_{2}$ & $\Delta$ & $1+1 / \Delta$ \\
\hline 3 & 1.7572064 & 0.7865396 & 0.42593834 & 3.3477577 \\
4 & 2.0365506 & 0.7386475 & 0.42591392 & 3.3478923 \\
5 & 2.2832894 & 0.7035557 & 0.42587323 & 3.3481166 \\
6 & 2.50674 & 0.67616 & 0.42582453 & 3.3483852 \\
\hline
\end{tabular}

Table A.1: $\gamma$ and $\Delta$ for different $l^{D}$.

The latest result for $\beta_{c}, \gamma$, and $\lambda_{i}$ with $b^{D}=2,3,4,5,6,7$, and 8 are obtained from $\beta_{c}$ which has accuracy up to $10^{-64}$. This has been checked independently by Dr. Bugra Oktay. 


\begin{tabular}{ccc}
\hline \hline$l^{D}$ & $\gamma_{c}$ & $\beta_{c}$ \\
\hline 2 & 1.29914073 & 1.17903017 \\
3 & 1.2992289425 & 1.95360681158131 \\
4 & 1.2993834469 & 2.57436660736656 \\
5 & 1.2995837185 & 3.11114443002212 \\
6 & 1.2998 & 3.5936743173223 \\
\hline
\end{tabular}

Table A.2: Critical values for different $l^{D}$.

All the digits shown for $\beta_{c}$ are exact. The digits for $\gamma$ and $\lambda$ are just for reference. 


\begin{tabular}{cccc}
\hline \hline$l_{\text {max }}$ & aim & $t_{\text {val }}$ & $\beta_{c}$ \\
\hline 32 & 40 & 64 & 1.953606811581309802176259103054353836796 \\
32 & 40 & 70 & 1.953606811581309802176259103054353836796 \\
32 & 40 & 76 & 1.953606811581309802176259103054353836796 \\
32 & 40 & 82 & 1.953606811581309802176259103054353836796 \\
32 & 40 & 88 & 1.953606811581309802176259103054353836796 \\
32 & 40 & 94 & 1.953606811581309802176259103054353836796 \\
36 & 40 & 72 & 1.953606811581309802176259103053851550042 \\
36 & 40 & 79 & 1.953606811581309802176259103053851550042 \\
36 & 40 & 86 & 1.953606811581309802176259103053851550042 \\
36 & 40 & 93 & 1.953606811581309802176259103053851550042 \\
36 & 40 & 100 & 1.953606811581309802176259103053851550042 \\
36 & 40 & 107 & 1.953606811581309802176259103053851550042 \\
\hline
\end{tabular}

Table A.3: $\beta_{c}$ for $l^{D}=3$. $l_{\max }$ is the initial and final truncation value; aim is the precision of $\beta_{c}$; $t_{v a l}$ is the intermediate truncation value. 


\begin{tabular}{|c|c|c|c|}
\hline$l_{\max }$ & aim & $t_{\text {val }}$ & $\beta_{c}$ \\
\hline 40 & 40 & 80 & 1.953606811581309802176259103053851542680 \\
\hline 40 & 40 & 88 & 1.953606811581309802176259103053851542680 \\
\hline 40 & 40 & 96 & 1.953606811581309802176259103053851542680 \\
\hline 40 & 40 & 104 & 1.953606811581309802176259103053851542680 \\
\hline 40 & 40 & 112 & 1.953606811581309802176259103053851542680 \\
\hline 40 & 40 & 120 & 1.953606811581309802176259103053851542680 \\
\hline 44 & 40 & 88 & 1.953606811581309802176259103053851542680 \\
\hline 44 & 40 & 97 & 1.953606811581309802176259103053851542679 \\
\hline 44 & 40 & 106 & 1.953606811581309802176259103053851542679 \\
\hline 44 & 40 & 115 & 1.953606811581309802176259103053851542680 \\
\hline 44 & 40 & 124 & 1.953606811581309802176259103053851542680 \\
\hline 48 & 40 & 96 & 1.953606811581309802176259103053851542679 \\
\hline 48 & 40 & 106 & 1.953606811581309802176259103053851542679 \\
\hline 48 & 40 & 116 & 1.953606811581309802176259103053851542680 \\
\hline 48 & 40 & 126 & 1.953606811581309802176259103053851542680 \\
\hline 48 & 40 & 136 & 1.953606811581309802176259103053851542679 \\
\hline 44 & 44 & 88 & 1.953606811581309802176259103053851542679 \\
\hline
\end{tabular}

Table A.4: $\beta_{c}$ for $l^{D}=3$. $l_{\max }$ is the initial and final truncation value; aim is the precision of $\beta_{c} ; t_{v a l}$ is the intermediate truncation value. 


\begin{tabular}{cccc}
\hline \hline$l_{\text {max }}$ & aim & $t_{\text {val }}$ & $\gamma$ \\
\hline 32 & 40 & 64 & 1.299228942539096704751713479952837548239 \\
32 & 40 & 70 & 1.299228942538789997108335021215099666667 \\
32 & 40 & 76 & 1.299228942538615134568811785928293767316 \\
32 & 40 & 82 & 1.299228942538635863061188181038703171005 \\
32 & 40 & 88 & 1.299228942539059636165227923786573772275 \\
32 & 40 & 94 & 1.299228942538904499736457717354803732439 \\
36 & 40 & 72 & 1.299228942538525734311222716384997381804 \\
36 & 40 & 79 & 1.299228942538584986703836967414504578432 \\
36 & 40 & 86 & 1.299228942539040279508918499040205580723 \\
36 & 40 & 93 & 1.299228942538599666527978413932297962763 \\
36 & 40 & 100 & 1.299228942538580056290327303744108375970 \\
36 & 40 & 107 & 1.299228942538615900535154791106016243179 \\
\hline
\end{tabular}

Table A.5: $\gamma$ for $l^{D}=3$. $l_{\max }$ is the initial and final truncation value; aim is the precision of $\beta_{c}$; $t_{v a l}$ is the intermediate truncation value. 


\begin{tabular}{|c|c|c|c|}
\hline$l_{\max }$ & aim & $t_{\text {val }}$ & $\gamma$ \\
\hline 40 & 40 & 80 & 1.299228942538560512974344369671636794173 \\
\hline 40 & 40 & 88 & 1.299228942538997274114849595551652796182 \\
\hline 40 & 40 & 96 & 1.299228942538548445718520535494144766115 \\
\hline 40 & 40 & 104 & 1.299228942538537050896258421727505797021 \\
\hline 40 & 40 & 112 & 1.299228942538591426805662193566184090402 \\
\hline 40 & 40 & 120 & 1.299228942539051596097055241722107819011 \\
\hline 44 & 40 & 88 & 1.299228942538952305920170000502989354029 \\
\hline 44 & 40 & 97 & 1.299228942539069502598778321768812111667 \\
\hline 44 & 40 & 106 & 1.299228942539194839714669287059491647935 \\
\hline 44 & 40 & 115 & 1.299228942538473931879181756076576634668 \\
\hline 44 & 40 & 124 & 1.299228942538880749149786615545974693001 \\
\hline 48 & 40 & 96 & 1.299228942539095015164526158475210808315 \\
\hline 48 & 40 & 106 & 1.299228942539165246170459726584161677860 \\
\hline 48 & 40 & 116 & 1.299228942538602536437857185472522101024 \\
\hline 48 & 40 & 126 & 1.299228942538603869472061544030207426767 \\
\hline 48 & 40 & 136 & 1.299228942539043022801978917931281023475 \\
\hline 44 & 44 & 88 & 1.299228942538617960143482614595703213070 \\
\hline
\end{tabular}

Table A.6: $\gamma$ for $l^{D}=3$. $l_{\max }$ is the initial and final truncation value; aim is the precision of $\beta_{c} ; t_{v a l}$ is the intermediate truncation value. 


\begin{tabular}{cccc}
\hline \hline$l_{\text {max }}$ & aim & $t_{\text {val }}$ & $\lambda_{1}$ \\
\hline 32 & 40 & 64 & 1.757206398881104776634947198492592259406 \\
32 & 40 & 70 & 1.757206398881338622230979649774077358276 \\
32 & 40 & 76 & 1.757206398881471944095176303096928190400 \\
32 & 40 & 82 & 1.757206398881456139902619438635159199953 \\
32 & 40 & 88 & 1.757206398881133039137978780437636844533 \\
32 & 40 & 94 & 1.757206398881251321063569226992441832437 \\
36 & 40 & 72 & 1.757206398881540106258633017023288673202 \\
36 & 40 & 79 & 1.757206398881494929976377339437821323472 \\
36 & 40 & 86 & 1.757206398881147797390437087766913471547 \\
36 & 40 & 93 & 1.757206398881483737518999371120100046028 \\
36 & 40 & 100 & 1.757206398881498689111536654311404640191 \\
36 & 40 & 107 & 1.757206398881471360093245187418656220222 \\
\hline
\end{tabular}

Table A.7: $\lambda_{1}$ for $l^{D}=3$. $l_{\max }$ is the initial and final truncation value; aim is the precision of $\beta_{c}$; $t_{v a l}$ is the intermediate truncation value. 


\begin{tabular}{|c|c|c|c|}
\hline$l_{\max }$ & aim & $t_{v a l}$ & $\lambda_{1}$ \\
\hline 40 & 40 & 80 & 1.757206398881513589680443715631324328457 \\
\hline 40 & 40 & 88 & 1.757206398881180586341823742384974826926 \\
\hline 40 & 40 & 96 & 1.757206398881522790216170569736413107470 \\
\hline 40 & 40 & 104 & 1.757206398881531478062923322335495287328 \\
\hline 40 & 40 & 112 & 1.757206398881490019797311562319092120003 \\
\hline 40 & 40 & 120 & 1.757206398881139169192363052790607742378 \\
\hline 44 & 40 & 88 & 1.757206398881214871807198913757615008738 \\
\hline 44 & 40 & 97 & 1.757206398881125516593027202997292128121 \\
\hline 44 & 40 & 106 & 1.757206398881029954799236710195417258758 \\
\hline 44 & 40 & 115 & 1.757206398881579602407027375276335310787 \\
\hline 44 & 40 & 124 & 1.757206398881269429415989292009118082317 \\
\hline 48 & 40 & 96 & 1.757206398881106064840609488858982612833 \\
\hline 48 & 40 & 106 & 1.757206398881052518045160956292319459983 \\
\hline 48 & 40 & 116 & 1.757206398881481549390334663903625197793 \\
\hline 48 & 40 & 126 & 1.757206398881480533034254925024151311184 \\
\hline 48 & 40 & 136 & 1.757206398881145705799260099596530545119 \\
\hline 44 & 44 & 88 & 1.757206398881469789769363759750127218183 \\
\hline
\end{tabular}

Table A.8: $\lambda_{1}$ for $l^{D}=3$. $l_{\max }$ is the initial and final truncation value; aim is the precision of $\beta_{c} ; t_{v a l}$ is the intermediate truncation value. 


\begin{tabular}{cccc}
\hline \hline$l_{\text {max }}$ & aim & $t_{\text {val }}$ & $\lambda_{2}$ \\
\hline 32 & 40 & 64 & 0.7865396437135632124646513820461519491398 \\
32 & 40 & 70 & 0.7865396437137473349053485006524538461584 \\
32 & 40 & 76 & 0.7865396437137582088903109454796719126079 \\
32 & 40 & 82 & 0.7865396437137181357413199409974844743448 \\
32 & 40 & 88 & 0.7865396437135465423920795604900117811111 \\
32 & 40 & 94 & 0.7865396437136659571574214805271695904763 \\
36 & 40 & 72 & 0.7865396437137944086620201717742327612720 \\
36 & 40 & 79 & 0.7865396437137387364931654600178421334067 \\
36 & 40 & 86 & 0.7865396437135860600599816895114634170213 \\
36 & 40 & 93 & 0.7865396437138244031794673946506184545514 \\
36 & 40 & 100 & 0.7865396437137724127159748242931991702469 \\
36 & 40 & 107 & 0.7865396437137262189270360569547320945806 \\
\hline
\end{tabular}

Table A.9: $\lambda_{2}$ for $l^{D}=3$. $l_{\max }$ is the initial and final truncation value; aim is the precision of $\beta_{c}$; $t_{v a l}$ is the intermediate truncation value. 


\begin{tabular}{|c|c|c|c|}
\hline$l_{\max }$ & aim & $t_{v a l}$ & $\lambda_{2}$ \\
\hline 40 & 40 & 80 & 0.7865396437137486463462731885157631508033 \\
\hline 40 & 40 & 88 & 0.7865396437136034737172745593040480607004 \\
\hline 40 & 40 & 96 & 0.7865396437138297283746857789288758433631 \\
\hline 40 & 40 & 104 & 0.7865396437137898263732676997543989045536 \\
\hline 40 & 40 & 112 & 0.7865396437137361287801437848213971505008 \\
\hline 40 & 40 & 120 & 0.7865396437135814777699735559420856758198 \\
\hline 44 & 40 & 88 & 0.7865396437136465995740842830943387319001 \\
\hline 44 & 40 & 97 & 0.7865396437135425472957373162902920662189 \\
\hline 44 & 40 & 106 & 0.7865396437134917960439593542434177736157 \\
\hline 44 & 40 & 115 & 0.7865396437138403018244167119981935273125 \\
\hline 44 & 40 & 124 & 0.7865396437136951727405705670621413629338 \\
\hline 48 & 40 & 96 & 0.7865396437135638966088823900835585835474 \\
\hline 48 & 40 & 106 & 0.7865396437135037790020999267775010197373 \\
\hline 48 & 40 & 116 & 0.7865396437137633101000482823479583475013 \\
\hline 48 & 40 & 126 & 0.7865396437138348258652061309343931866066 \\
\hline 48 & 40 & 136 & 0.7865396437135849492512972835102014507718 \\
\hline 44 & 44 & 88 & 0.7865396437138697473782302056330013043077 \\
\hline
\end{tabular}

Table A.10: $\lambda_{2}$ for $l^{D}=3$. $l_{\max }$ is the initial and final truncation value; aim is the precision of $\beta_{c} ; t_{v a l}$ is the intermediate truncation value. 


\begin{tabular}{cccc}
\hline \hline$l_{\text {max }}$ & aim & $t_{\text {val }}$ & $\beta_{c}$ \\
\hline 32 & 40 & 64 & 2.574366607366560276233393764322237051524 \\
32 & 40 & 128 & 2.574366607366560276233393764322237048475 \\
40 & 40 & 80 & 2.574366607366560276233393764322236128484 \\
\hline
\end{tabular}

Table A.11: $\beta_{c}$ for $l^{D}=4$. $l_{\max }$ is the initial and final truncation value; aim is the precision of $\beta_{c} ; t_{v a l}$ is the intermediate truncation value.

\begin{tabular}{cccc}
\hline \hline$l_{\text {max }}$ & aim & $t_{\text {val }}$ & $\gamma$ \\
\hline 32 & 40 & 64 & 1.299383446968990648082420597389185027744 \\
32 & 40 & 128 & 1.299383446969440744025709126999082084684 \\
40 & 40 & 80 & 1.299383446969352427087654937834492881873 \\
\hline
\end{tabular}

Table A.12: $\gamma$ for $l^{D}=4$. $l_{\max }$ is the initial and final truncation value; aim is the precision of $\beta_{c} ; t_{v a l}$ is the intermediate truncation value.

\begin{tabular}{cccc}
\hline \hline$l_{\text {max }}$ & aim & $t_{v a l}$ & $\lambda_{1}$ \\
\hline 32 & 40 & 64 & 2.036550640841265654441039283550142975455 \\
32 & 40 & 128 & 2.036550640840763901588513926490994222893 \\
40 & 40 & 80 & 2.036550640840862354543391085542627312959 \\
\hline
\end{tabular}

Table A.13: $\lambda_{1}$ for $l^{D}=4$. $l_{\max }$ is the initial and final truncation value; aim is the precision of $\beta_{c} ; t_{v a l}$ is the intermediate truncation value.

\begin{tabular}{cccc}
\hline \hline$l_{\text {max }}$ & aim & $t_{\text {val }}$ & $\lambda_{2}$ \\
\hline 32 & 40 & 64 & 0.7386474756161715818979884554824340680415 \\
32 & 40 & 128 & 0.7386474756159031757269478294318271174266 \\
40 & 40 & 80 & 0.7386474756159942496409988023234663687984 \\
\hline
\end{tabular}

Table A.14: $\lambda_{2}$ for $l^{D}=4$. $l_{\max }$ is the initial and final truncation value; aim is the precision of $\beta_{c} ; t_{v a l}$ is the intermediate truncation value. 


\begin{tabular}{cccc}
\hline \hline$l_{\text {max }}$ & aim & $t_{v a l}$ & $\beta_{c}$ \\
\hline 16 & 40 & 32 & 3.111144430022622778922915294803883518779 \\
16 & 40 & 44 & 3.111144430022123178568317034525175980765 \\
16 & 40 & 56 & 3.111144430022123178453278705116288715924 \\
16 & 40 & 68 & 3.111144430022123178453278700620123711324 \\
16 & 40 & 80 & 3.111144430022123178453278700620123700812 \\
20 & 40 & 40 & 3.111144430022121482846073290482461542807 \\
20 & 40 & 55 & 3.111144430022121460055934935111232744852 \\
20 & 40 & 70 & 3.11114443002212146005593491051657647209 \\
20 & 40 & 85 & 3.11114443002212146005593491051657646914 \\
20 & 40 & 100 & 3.11114443002212146005593491051657646914 \\
24 & 40 & 48 & 3.111144430022121460029837242613994484801 \\
24 & 40 & 66 & 3.111144430022121460029336989460676552477 \\
\hline
\end{tabular}

Table A.15: $\beta_{c}$ for $l^{D}=5$. $l_{\max }$ is the initial and final truncation value; aim is the precision of $\beta_{c} ; t_{v a l}$ is the intermediate truncation value. 


\begin{tabular}{cccc}
\hline \hline$l_{\text {max }}$ & aim & $t_{\text {val }}$ & $\gamma$ \\
\hline 16 & 40 & 32 & 1.299583719032215803258708342850073978291 \\
16 & 40 & 44 & 1.299583718512967899988772557264971016965 \\
16 & 40 & 56 & 1.299583718513015961760844982956631026984 \\
16 & 40 & 68 & 1.299583718513000258998879319229193303839 \\
16 & 40 & 80 & 1.299583718512884449786487925987916981462 \\
20 & 40 & 40 & 1.299583718510391233336485024341542227967 \\
20 & 40 & 55 & 1.299583718510885508385786028682654337013 \\
20 & 40 & 70 & 1.299583718510860503268783721605417769867 \\
20 & 40 & 85 & 1.299583718510772405623681749852427845554 \\
20 & 40 & 100 & 1.299583718510866691792733336514085761198 \\
24 & 40 & 48 & 1.299583718510494428821083077019940044704 \\
24 & 40 & 66 & 1.299583718510317145590856700363726361095 \\
\hline
\end{tabular}

Table A.16: $\gamma$ for $l^{D}=5$. $l_{\max }$ is the initial and final truncation value; aim is the precision of $\beta_{c} ; t_{v a l}$ is the intermediate truncation value. 


\begin{tabular}{cccc}
\hline \hline$l_{\text {max }}$ & aim & $t_{\text {val }}$ & $\lambda_{1}$ \\
\hline 16 & 40 & 32 & 2.283289466193888103702553954133792956019 \\
16 & 40 & 44 & 2.283289466947087857534438970978204392471 \\
16 & 40 & 56 & 2.283289466947018141095169520848258742954 \\
16 & 40 & 68 & 2.283289466947040918878920234229090506686 \\
16 & 40 & 80 & 2.283289466947208906982029741595735355549 \\
20 & 40 & 40 & 2.28328946695082546471029663501288085322 \\
20 & 40 & 55 & 2.283289466950108489555796570981572785119 \\
20 & 40 & 70 & 2.283289466950144760954992961127449982234 \\
20 & 40 & 85 & 2.283289466950272551792902701444930541026 \\
20 & 40 & 100 & 2.283289466950135784135464694263289243455 \\
24 & 40 & 48 & 2.283289466950675773564409213192212199554 \\
24 & 40 & 66 & 2.283289466950932933361493133742513498114 \\
\hline
\end{tabular}

Table A.17: $\lambda_{1}$ for $l^{D}=5$. $l_{\max }$ is the initial and final truncation value; aim is the precision of $\beta_{c}$; $t_{v a l}$ is the intermediate truncation value. 


\begin{tabular}{cccc}
\hline \hline$l_{\text {max }}$ & aim & $t_{\text {val }}$ & $\lambda_{2}$ \\
\hline 16 & 40 & 32 & 0.7035557328641656688813538819335051668311 \\
16 & 40 & 44 & 0.7035557367803160850675204604737219646894 \\
16 & 40 & 56 & 0.7035557367802940352394934374136841685974 \\
16 & 40 & 68 & 0.7035557367803026871247587308613526214851 \\
16 & 40 & 80 & 0.7035557367804147050569049496755473998989 \\
20 & 40 & 40 & 0.703555736808953377405725899832537745963 \\
20 & 40 & 55 & 0.7035557368091700380359497409605427778522 \\
20 & 40 & 70 & 0.7035557368091838153216202672322253857599 \\
20 & 40 & 85 & 0.70355573680928056477538767565833223638 \\
20 & 40 & 100 & 0.7035557368091804055777899888779504020749 \\
24 & 40 & 48 & 0.7035557368094688695865932830722341836117 \\
24 & 40 & 66 & 0.7035557368095326614060477192309255003617 \\
\hline
\end{tabular}

Table A.18: $\lambda_{2}$ for $l^{D}=5$. $l_{\max }$ is the initial and final truncation value; aim is the precision of $\beta_{c}$; $t_{v a l}$ is the intermediate truncation value. 


\section{APPENDIX B RESCALING OF THE ERG EQUATION}

In the following, we will show that the equation (B.1) is invariant under some rescaling.

$$
-3 u+\rho u^{\prime}+\frac{1}{1+u^{\prime}+2 \rho u^{\prime \prime}}=0
$$

\section{B.1 No $A$ Dependence}

Let us start with the following equation:

$$
-3 u+\rho u^{\prime}+\frac{A}{1+u^{\prime}+2 \rho u^{\prime \prime}}=0
$$

We will show that the prefactor A can be eliminated after the rescaling of $\rho$ and $u$.

$$
-3 \frac{u}{A}+\rho \frac{u^{\prime}}{A}+\frac{1}{1+u^{\prime}+2 \rho u^{\prime \prime}}=0
$$

We first rescale the variable $\rho$

$$
\begin{aligned}
& \alpha \equiv \frac{\rho}{A} \\
& u^{\prime} \equiv \frac{\partial u}{\partial \rho}=\frac{1}{A} \frac{\partial u}{\partial \alpha} \\
& u^{\prime \prime} \equiv \frac{\partial^{2} u}{\partial \rho^{2}}=\frac{1}{A^{2}} \frac{\partial^{2} u}{\partial \alpha^{2}} \\
& \Rightarrow \quad-3 \frac{u}{A}+\frac{\alpha}{A} \frac{\partial u}{\partial \alpha}+\frac{1}{1+\frac{1}{A} \frac{\partial u}{\partial \alpha}+\frac{2 \alpha}{A} \frac{\partial^{2} u}{\partial \alpha^{2}}}=0
\end{aligned}
$$

We further rescale the field $u$ 


$$
\begin{gathered}
v \equiv \frac{u}{A} \\
\frac{1}{A} \frac{\partial u}{\partial \alpha}=\frac{\partial v}{\partial \alpha} \\
\frac{1}{A} \frac{\partial^{2} u}{\partial \alpha^{2}}=\frac{\partial^{2} v}{\partial \alpha^{2}} \\
-3 v+\frac{\partial v}{\partial \alpha}+\frac{1}{1+\frac{\partial v}{\partial \alpha}+2 \alpha \frac{\partial v}{\partial \alpha}}=0 \\
-3 v+\alpha v^{\prime}+\frac{1}{1+v^{\prime}+2 \alpha v^{\prime \prime}}=0
\end{gathered}
$$

Therefore, the coefficient A has no effect on the critical exponents $\nu$ and $\omega$.

Note:

$$
\alpha \equiv \frac{\rho}{A} \quad v \equiv \frac{u}{A} \Rightarrow\left\{\begin{aligned}
& \rho \rightarrow A \alpha \\
& u \rightarrow A v \\
& u^{\prime} \rightarrow v^{\prime} \\
& u^{\prime \prime} \rightarrow \frac{1}{A} v^{\prime \prime}=\frac{1}{A} \frac{\partial^{2} v}{\partial \alpha^{2}}
\end{aligned}\right.
$$

\section{B.2 No $B$ Dependence}

$$
-3 u+\rho u^{\prime}+\frac{1}{B+u^{\prime}+2 \rho u^{\prime \prime}}=0
$$

By the same reasoning, we can prove that the factor $B$ has no effect on the critical exponents. We can do the following substitution:

$$
\alpha \equiv \rho B^{2} \quad v \equiv u B \Rightarrow\left\{\begin{aligned}
\rho & \rightarrow \frac{\alpha}{B^{2}} \\
u & \rightarrow \frac{v}{B} \\
u^{\prime} & \rightarrow B v^{\prime} \\
u^{\prime \prime} & \rightarrow B^{3} v^{\prime \prime}=B^{3} \frac{\partial^{2} v}{\partial \alpha^{2}}
\end{aligned}\right.
$$




$$
-3 v+\alpha v^{\prime}+\frac{1}{1+v^{\prime}+2 \alpha v^{\prime \prime}}=0
$$

\section{B.3 More about $A$ and $B$ dependence}

Let us look at higher orders: $A \frac{1}{\left(1+u^{\prime}+2 \rho u^{\prime \prime}\right)^{2}}, \frac{1}{\left(B+u^{\prime}+2 \rho u^{\prime \prime}\right)^{2}}$

$$
\begin{array}{cc}
\alpha \equiv \frac{\rho}{A} & -3 u+\rho u^{\prime}+\frac{A}{\left(1+u^{\prime}+2 \rho u^{\prime \prime}\right)^{2}}=0 \\
\stackrel{v \equiv \frac{u}{A}}{ } & -3 \frac{u}{A}+\frac{\alpha}{A} \frac{\partial u}{\partial \alpha}+\frac{1}{\left(1+\frac{1}{A} \frac{\partial u}{\partial \alpha}+\frac{2 \alpha}{A} \frac{\partial^{2} u}{\partial \alpha^{2}}\right)^{2}}=0 \\
& -3 v+\alpha v^{\prime}+\frac{1}{\left(1+v^{\prime}+2 \alpha v^{\prime \prime}\right)^{2}}=0
\end{array}
$$

So that the coefficient A has no effect on the critical exponents $\nu$ and $\omega$ even for higher orders.

For the coefficient B, we will have

$$
\Rightarrow \begin{aligned}
& -3 u+\rho u^{\prime}+\frac{1}{\left(B+u^{\prime}+2 \rho u^{\prime \prime}\right)^{2}}=0 \\
& -3 v+\rho v^{\prime}+\frac{1}{B} \frac{1}{\left(1+v \prime+2 \rho v^{\prime \prime}\right)^{2}}=0
\end{aligned}
$$

and

$$
-3 v+\rho v^{\prime}+\frac{1}{B^{n-1}} \frac{1}{\left(1+v \prime+2 \rho v^{\prime \prime}\right)^{n}}=0
$$




\section{APPENDIX C LATTICE GAUGE THEORIES}

Quantum Chromodynamics (QCD) is a non-Abelian gauge theory and is the best candidate for a theory of strong interactions. For now, only QCD is able to explain the experimental fact that the constituents of hadrons behave almost as free at short distance (asymptotic freedom). At long distance, the strong interactions are really strong! The perturbation theory breaks down. We would like not only to understand why "colored" object, such as quark, are not visible (confinement) but also to compute the real particles spectrum. Lattice QCD is a promising approach in dealing with the above problems by regularizing QCD on the space time lattice. In the Lattice QCD, fermion fields reside on the sites and gauge fields reside on the links. The fermion fields carry the color quantum number of the gauge group $S U(3)$. At each site, there are fermion fields $\psi_{\alpha}$, where $\alpha$ is the color index. Gauge fields are the connections of the color coordinate.

Now consider a four-dimensional hyper-cubic lattice on the Euclidean space with lattice spacing $a$. $U_{\nu}(n)$ connects the neighboring lattice sites $n$ and $n+\hat{\mu}$.

$$
U_{\nu}(n)=\exp \left(i g a A_{\nu}^{c}(n) \lambda_{c}\right)
$$

where $\lambda_{c}$ stands for 8 generators of $\mathrm{SU}(3)$ and $g$ is the gauge coupling constant. 


$$
\lambda_{1}=\frac{1}{2}\left(\begin{array}{ccc}
0 & 1 & 0 \\
1 & 0 & 0 \\
0 & 0 & 0
\end{array}\right) \quad \lambda_{2}=\frac{1}{2}\left(\begin{array}{ccc}
0 & -i & 0 \\
i & 0 & 0 \\
0 & 0 & 1 \\
0 & 0 & 0
\end{array}\right) \quad \lambda_{3}=\frac{1}{2}\left(\begin{array}{ccc}
1 & 0 & 0 \\
0 & -1 & 0 \\
0 & 0 & 0 \\
0 & 0 & 0
\end{array}\right) \quad \lambda_{5}=\frac{1}{2}\left(\begin{array}{ccc}
0 \\
1 & 0 & 0
\end{array}\right) \quad \lambda_{6}=\frac{1}{2}\left(\begin{array}{ccc}
0 & 0 & 0 \\
0 & 0 & 0 \\
0 & 0 & 1 \\
0 & 0 & -i \\
0 & i & 0
\end{array}\right) \quad \lambda_{8}=\frac{1}{2 \sqrt{3}}\left(\begin{array}{ccc}
1 & 0 & 0 \\
0 & 0 & 0 \\
0 & 1 & 0 \\
0 & 0 & -2
\end{array}\right)
$$

The plaquette defined in the $\mu-\nu$ plane is :

$$
U_{\mu \nu}=U_{\mu}(n) U_{\nu}(n+\hat{\mu}) U_{\mu}^{\dagger}(n+\hat{\nu}) U_{\nu}^{\dagger}(n)
$$

Recall that the continuum Yang Mills actions $S_{Y M}$ is the following

$$
S_{Y M}=-\frac{1}{2} \int_{0}^{\beta} d \tau \int d^{3} x \operatorname{tr} F^{\mu \nu} F_{\mu \nu}, \quad F_{\mu \nu}^{a}=F_{\mu \nu}^{a} T^{a}=\partial_{\mu} A_{\nu}^{a}-\partial_{\nu} A_{\mu}^{a}-g f^{a b c} A_{\mu}^{b} A_{\nu}^{c}
$$

It can be written as

$$
S(U)=\beta \sum_{n} \sum_{\mu<\nu}\left(1-\frac{1}{3} \operatorname{Retr} U_{\mu \nu}(n)\right)
$$

on the lattice.

And the continuum free Euclidean fermion action is

$$
S=\int d^{d} x \bar{\psi}(\not \partial+m) \psi
$$

The naive lattice fermion action can be obtained by replacing the derivatives by symmetric differences:

$$
\partial_{\mu} \psi_{\alpha}(n)=\frac{1}{2 a}\left(\psi_{\alpha}(n+\hat{\mu})-\psi_{\alpha}(n-\hat{\mu})\right)
$$




$$
S_{\text {naive }}=\sum_{n, \mu} \bar{\psi}(n) \frac{\gamma_{\mu}}{2 a}(\psi(n+\hat{\mu})-\bar{\psi}(n-\hat{\mu}))+m \sum_{n} \bar{\psi}(n) \psi(n)
$$

The fermion propagator can be calculated as

$$
G(p)_{\text {naive }}=\left(m a+i \sum_{\mu} \gamma_{\mu} \sin \left(p_{\mu} a\right)\right)^{-1}
$$

However, there are sixteen poles for the propagator $G(p)_{\text {naive }}$ in the $m=0$ limit

$$
p=(0,0,0,0), \quad p=(0,0,0, \pi), \quad p=(0,0, \pi, 0), \quad \cdots \quad p=(\pi, \pi, \pi, \pi)
$$

This is a theory with 16 mass-less fermions instead of 1 ! And this is the so called fermion doubling problem: the number of mass-less fermions would be $2^{d}$ in a $d$ space-time dimensions.

One way the deal with the doublers is to reduce the number of fermion flavors by using one component "staggered" fermion fields instead of four component Dirac spinors.

The staggered field $\chi(n)$ is defined as

$$
\psi(n)=\eta_{\mu}(n) \chi(n), \quad \eta_{\mu}(n)=(-1)^{n_{1}+n_{2}+\cdots+n_{\mu-1}}
$$

The resulting lattice action for "staggered" fermion action is:

$$
S_{\text {stag }}=\frac{1}{2 a} \sum_{n, \mu} \eta_{\mu}(n) \bar{\chi}(n)\left[U_{\mu}(n) \chi(n+\hat{\mu})-U_{\mu}^{\dagger}(n-\hat{\mu}) \chi(n-\hat{\mu})\right]+m \sum_{n} \bar{\chi}(n) \chi(n)
$$

$S_{\text {stag }}$ is invariant under the chiral transformation as $m \rightarrow 0$.

There are also many different other fermion actions, such as Wilson fermion, Domain wall fermion, $\cdots$. We do not discuss them in details here. 
The expectation value of an observable is:

$$
<O>=\frac{\int D U O(U) e^{-S[U]}}{\int D U e^{-S[U]}}
$$

For a $10^{4}$ lattice, there are about $8 * 4 * 10^{4}=320000$ integrations to be done.

Importance sampling methods: select a sequence of gauge configurations with a probability distribution given by the Boltzmann factor $e^{-S(U)}$.

The Monte Carlo method: Metropolis method and heat bath method.

Molecular dynamics method.

The Metropolis method:

I, Change the gauge configuration $\phi \rightarrow \phi^{\prime}$

$$
P\left(\phi^{\prime} \leftarrow \phi\right)=P\left(\phi \leftarrow \phi^{\prime}\right)
$$

II, Choose a random number $r$ distributed uniformly on $[0,1]$.

III, Calculate $S(\phi)$ and $S\left(\phi^{\prime}\right)$.

IV, Always accept the change if $S(\phi)<S\left(\phi^{\prime}\right)$.

$\mathrm{V}$, Accept the change if $e^{-\Delta S}>r$.

Molecular dynamics method:

I, Define a new Hamiltonian: $H(p, \phi)=\sum_{n} \frac{p_{n}^{2}}{2}+S(\phi)$

where $p_{n}$ is the fictitious momentum conjugate to the field $\phi_{n}$.

II, $H(p, \phi)$ governs the dynamics under the "evolution time" $\tau$.

$$
\dot{\phi}_{n}=p_{n}, \quad \dot{p}_{n}=-\partial S / \partial \phi_{n}
$$

III, The above equations define a trajectory $[p(\tau), \phi(\tau)]$ through phase space.

Iv, The expectation value of an observable can be computed as:

$$
<O>=\frac{1}{T} \int_{\tau_{0}}^{\tau_{0}+T} D \tau O[\phi(\tau)]
$$

Molecular dynamics method for $\mathrm{SU}(\mathrm{N})$ pure gauge: 
Link variable $U_{\mu}(n)=e^{i A_{\mu}(n)}$ can be treated as coordinates.

The equation of motion for $U_{\mu}(n)$ :

$$
\dot{U}_{\mu}(n)=i H_{\mu}(n) U_{\mu}(n), \quad H_{\mu}(n)=\sum_{\alpha} h_{\mu}^{\alpha}(n) T^{\alpha}
$$

where $T^{\alpha}$ are generators of $\mathrm{SU}(\mathrm{N})$ Lie algebra and $h_{\mu}^{\alpha}(n)$ will be chosen from unit Gaussian random values.

We can also get

$$
i \dot{H}_{\mu}(n)=-\left.\frac{\beta}{N} \operatorname{Tr}\left(U_{\mu}(n) V_{\mu}(n)\right)\right|_{T A}
$$

Hybrid method(Metropolis+Molecular dynamics):

I, Choose the coordinates $\left\{\phi_{i}\right\}$ in some arbitrary way.

II, Choose the momenta $\left\{\pi_{i}\right\}$ from the Gaussian ensemble:

$$
P_{G}(\pi)=N_{o} e^{-\frac{1}{2} \sum \pi_{i}^{2}}
$$

III, Calculate $\tilde{\pi}$ according to :

$$
\tilde{\pi}_{i}=\pi_{i}(n)-\frac{\epsilon}{2} \frac{\partial S[\phi]}{\partial \phi_{i}(n)}
$$

IV, Iterate the following form $n$ times:

$$
\phi_{i}(n+1)=\phi_{i}(n)+\epsilon \tilde{\pi}_{i}(n), \quad \tilde{\pi}_{i}(n+1)=\tilde{\pi}_{i}(n)-\epsilon \frac{\partial S[\phi]}{\partial \phi_{i}(n+1)}
$$

$\mathrm{V}$, Accept $\left\{\phi_{i}^{\prime}, \pi_{i}^{\prime}\right\}$ with probability: $p=\min \left(1, e^{-H\left[\phi^{\prime}, \pi^{\prime}\right]} / e^{-H[\phi, \pi]}\right)$.

Lattice Gauge Theories An Introduction, Heinz J. Rothe, page: 305.

The Dirac fields:

I, Grassmann valued:

$$
\left\{\eta_{i}, \eta_{j}\right\}=\eta_{i} \eta_{j}+\eta_{j} \eta_{i}=0
$$


II, The Dirac fields, $\psi$, enter quadratically into the action $S$.

$$
S=\bar{\psi} M \psi+\cdots
$$

The partition function for full QCD with one fermion flavor is:

$$
Z=\int[d U][d \bar{\psi}][d \psi] \exp \left[-S_{G}(U)-\bar{\psi} M(U) \psi\right], \quad M=D+m
$$

Integrating over Grassmann variables:

$$
Z=\int[d U] \exp \left[-S_{G}(U)\right] \operatorname{det} M(U)
$$

Introducing a pseudo-fermion field $\Phi$ :

$$
\operatorname{det} M(U)=\int\left[d \Phi^{*} d \Phi\right] \exp \left[-\Phi^{*} M^{-1} \Phi\right]
$$

The pseudonym action can be written as:

$$
\left.Z=\int[d U]\left[d \Phi^{*}\right][d \Phi] \exp \left[-S_{G}(U)\right]-\Phi^{*} M(U) \Phi\right]
$$

For 2 flavors, one would have:

$$
Z=\int[d U] \exp \left[-S_{G}(U)\right] \operatorname{det}\left[M^{\dagger} M\right], \quad \operatorname{det} D=\operatorname{det} D^{\dagger}
$$

For staggered fermion:

$$
\begin{gathered}
Z=\int[d U] \exp \left[-S_{G}(U)\right] \prod_{f} \operatorname{det}\left[M^{\dagger} M\right]^{N_{f} / 4} \\
\left(M^{\dagger} M\right)^{-N_{f} / 4} \approx r\left(M^{\dagger} M\right)=a_{0}+\sum_{n}=1^{N} \frac{a_{n}}{\left(M^{\dagger} M\right)+b_{n}}
\end{gathered}
$$

where $a_{n}$ and $b_{n}$ are constants.

Rational approximation for $x^{1 / 8}$ : 


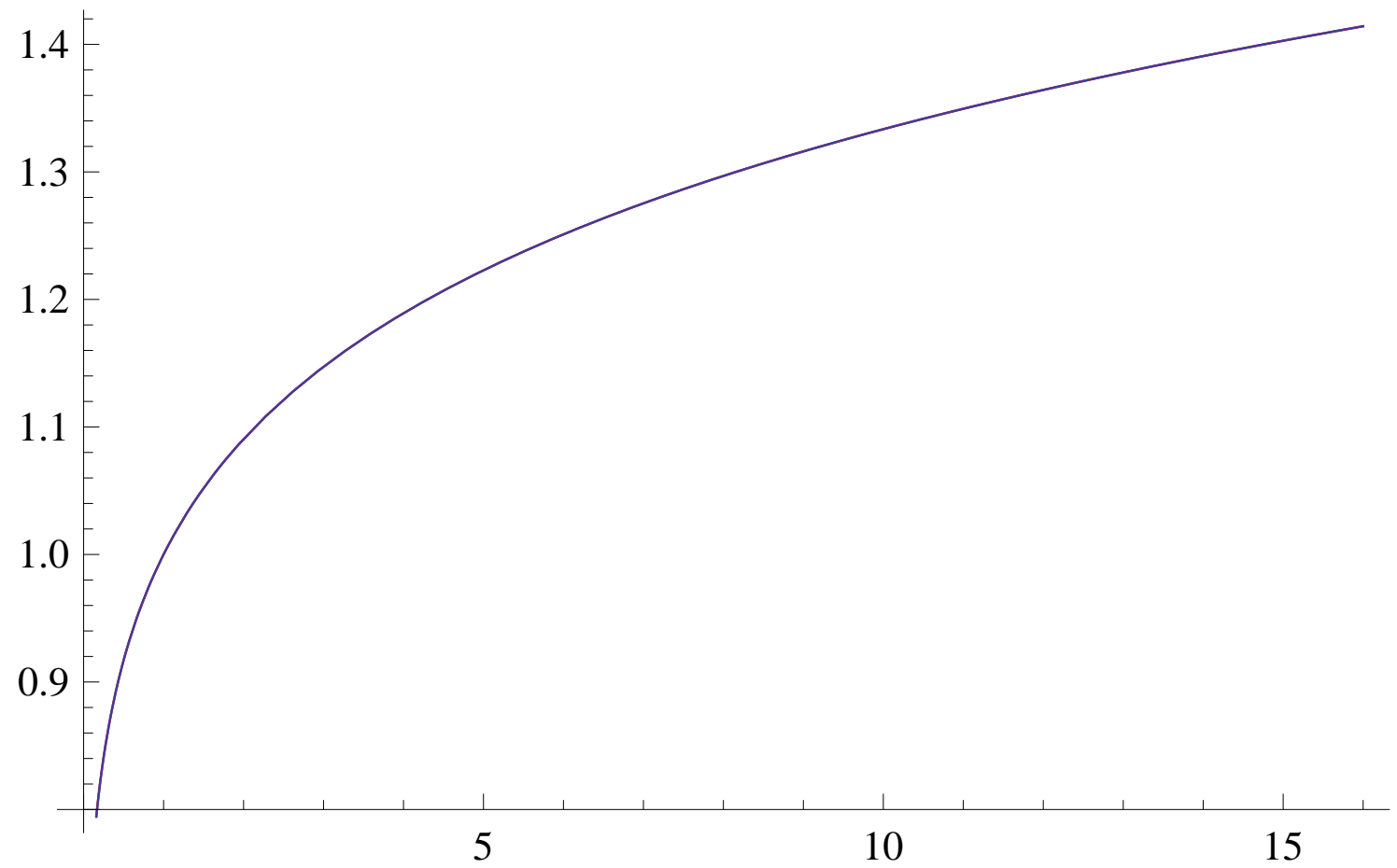

Figure C.1: Test for the rational approximation.

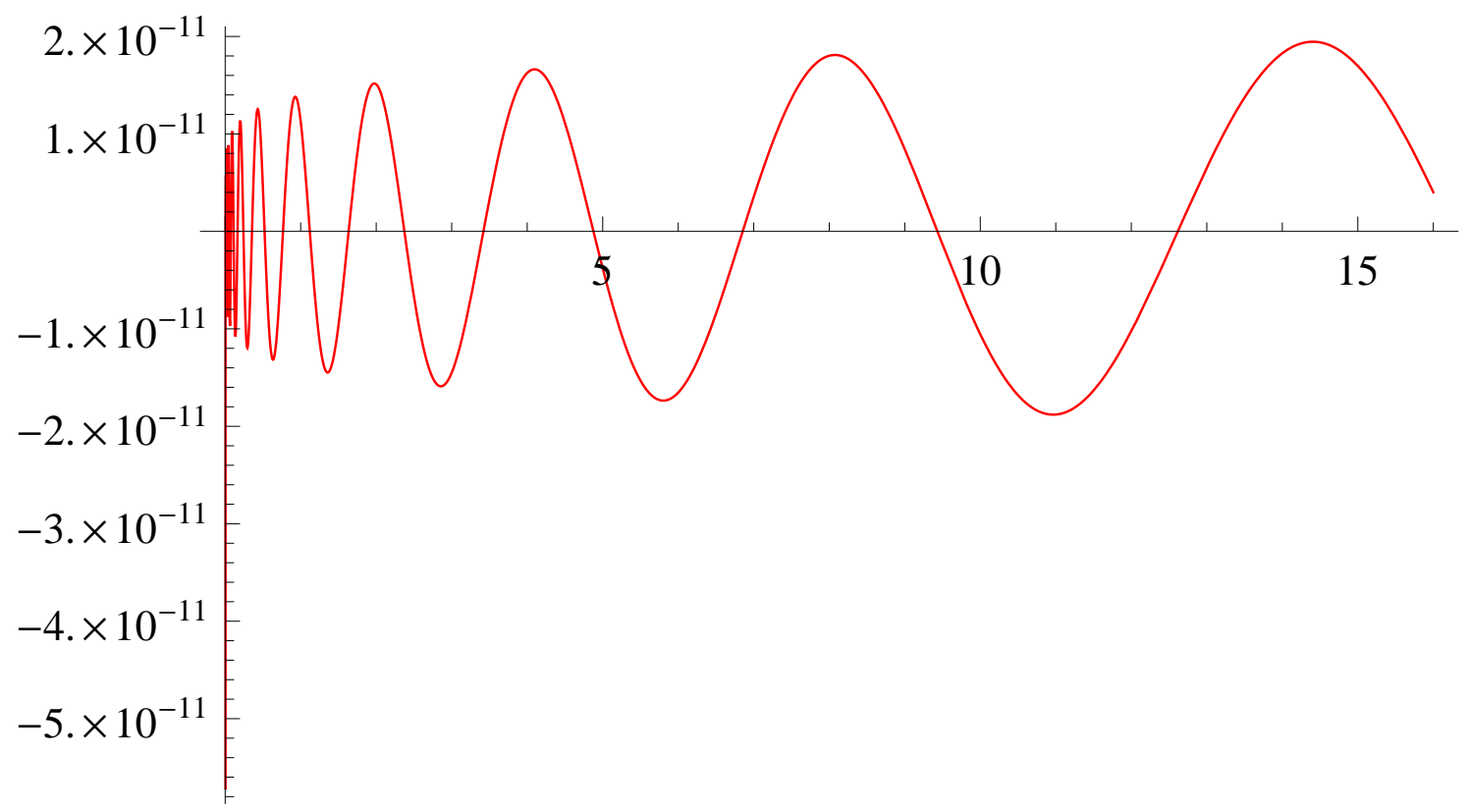

Figure C.2: Test for the rational approximation. 


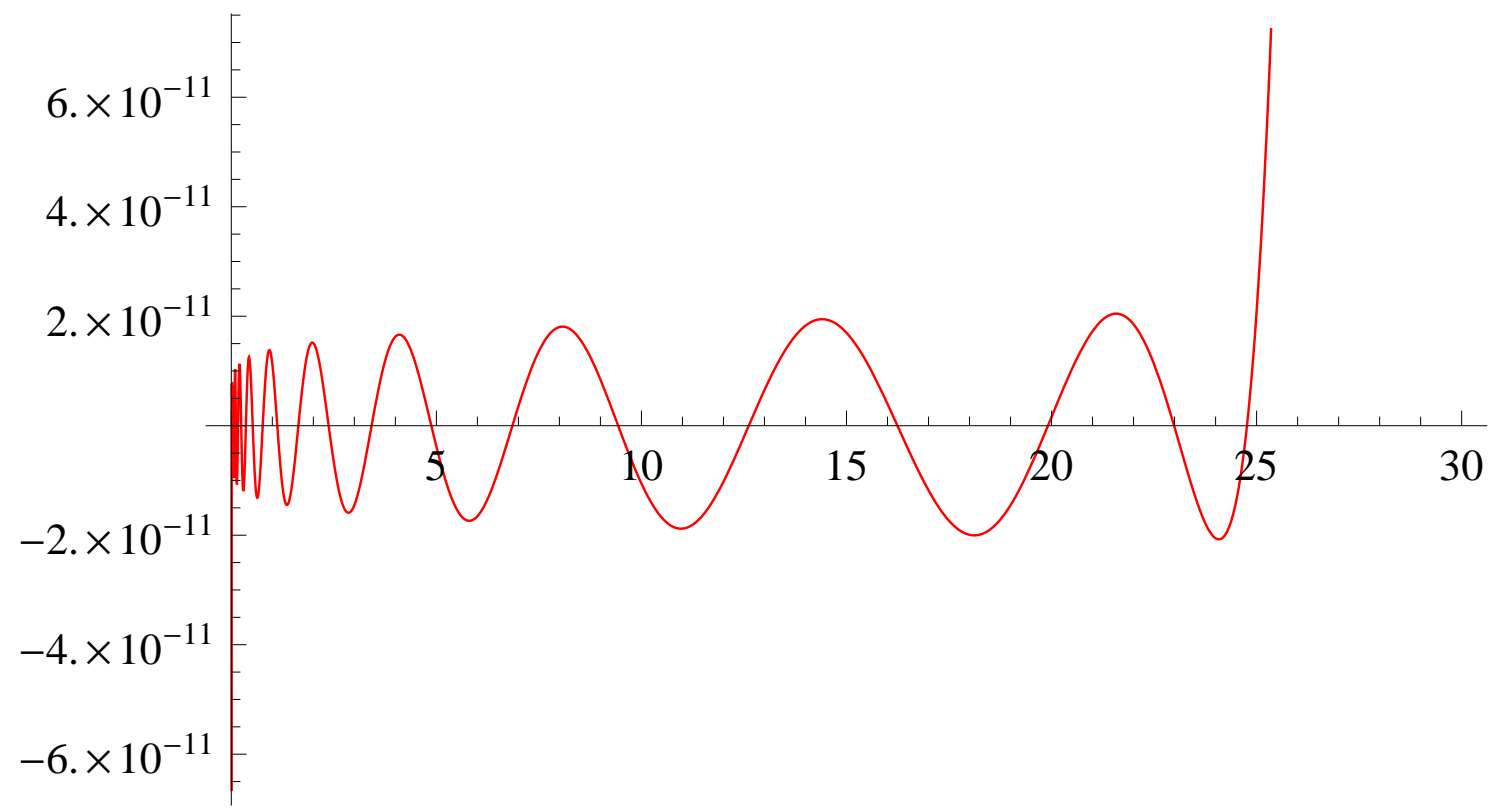

Figure C.3: Test for the rational approximation.

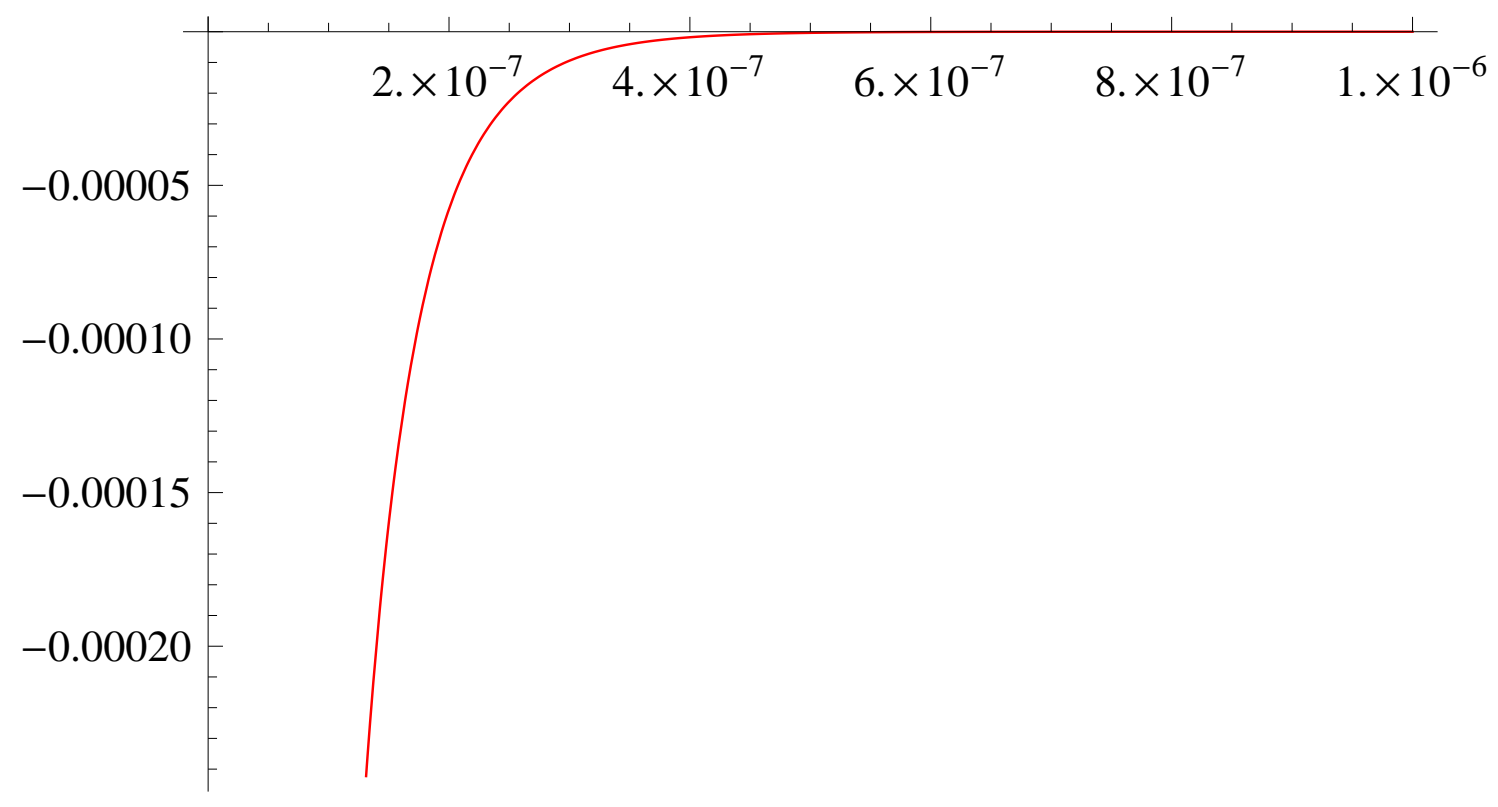

Figure C.4: Test for the rational approximation. 


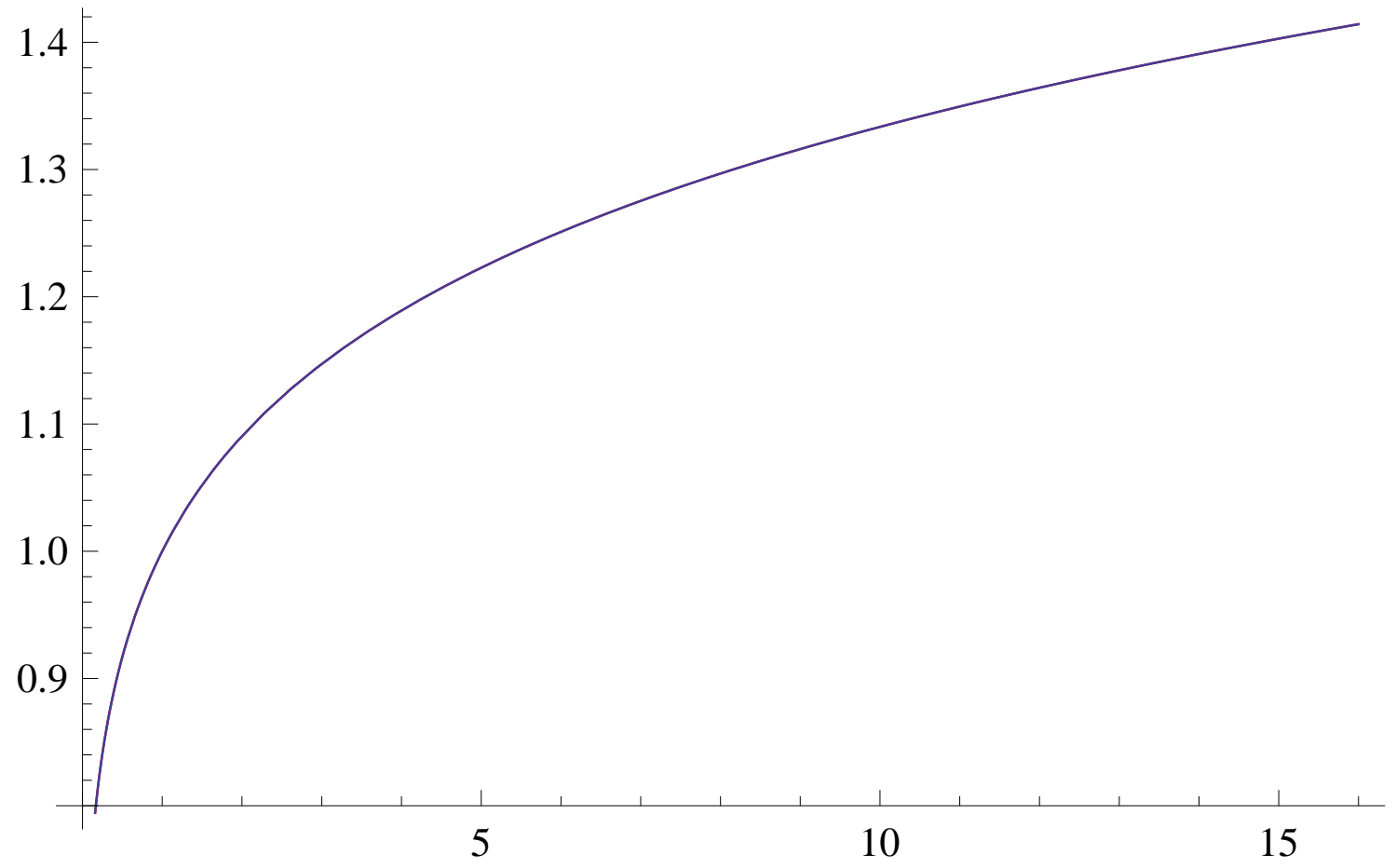

Figure C.5: Test for the rational approximation. 


\section{APPENDIX D ERROR ANALYSIS OF THE BINDER CUMULANT}

In this appendix, we presented several methods on calculating the error of the Binder Cumulant.

\section{D.1 What We Have}

$4 \times N_{\sigma}{ }^{3}$ lattices for a pure $S U(2)$ gauge theory with $N_{\sigma}=2,4,6,8,10,12,14,16$. The $\beta$ range decreases with increasing volume. We generated 50,000 Polyakov loops with 20 different seeds for each $\beta$.

\section{D.2 What We Want}

Calculate the Binder cumulant $g_{4}=1-\frac{<P^{4}>}{\left.3<P^{2}\right\rangle^{2}}$ and corresponding errors.

\section{D.3 What We Have Done}

- Error propagation method. First calculate the $m^{2}$ and $m^{4}$ and the corresponding errors of them. Then use error propagation to calculate $g_{4}$. The error propagation formula is $\left(\left(1 /\left(3<m 2>^{2}\right)\right)^{2} * e r r m 4^{2}+((2 *<m 4>\right.$ )$\left.\left./\left(3<m 2>^{3}\right)\right)^{2} * e r r m 2^{2}\right)^{0} .5$.

- Bootstrap method. We then tried bootstrap method. We first combine all the $5000 \times 20=100000$ data and then randomly pick 20000 out in order to cancel out the correlation. And then calculate $g_{4}$ from the 20000 data. We repeat the above procedure 20 times and then calculate the average and error from the calculated $20 g_{4}$.

- We also calculated the correlation time for one particular set of data (50000). It is around 10 for $N_{\sigma}=4$ and 200 for $N_{\sigma}=16$.

- We fit the 20 ( or less) different $g_{4}(\beta)$ s by following Numerical Recipes in $C++$ page 66\%. : 


\section{D.4 The Proper Way Of Getting The Error}

- Use data from 20 different seeds to calculate $20 g_{4}$. We can simply ignore the correlation among data since what we want is the average value. $\left\langle P^{4}\right\rangle$ is unbiased and $<P^{2}>^{2}$ is biased.

- Jackknife $20 g_{4}$ s to deal with the biased $<P^{2}>^{2}$ and the correlation between $<P^{4}>$ and $<P^{2}>^{2}$. We will get the error. If we want to get the $2 \sigma$ error, then we need to multiply by a factor. See Berg's book on page 78.

- Do the same for the rest of the $\beta$.

- Do the fitting according to Numerical Recipes in $C++$ page $66 \%$. 


\section{REFERENCES}

[1] A. Denbleyker, A. Bazavov, D. Du, Y. Liu, Y. Meurice, et al., "Fisher's zeros, complex RG flows and confinement in LGT models," PoS, vol. LATTICE2011, p. 299, 2011.

[2] D. B. Kaplan, J.-W. Lee, D. T. Son, and M. A. Stephanov, "Conformality lost," Phys. Rev. D, vol. 80, p. 125005, Dec 2009.

[3] D. F. Litim, "Towards functional flows for hierarchical models," Phys. Rev. D, vol. 76, p. 105001, Nov 2007.

[4] E. C. Stueckelberg and A. Petermann, "The normalization group in quantum theory," Helv.Phys.Acta, vol. 24, pp. 317-319, 1951.

[5] E. Stueckelberg and A. Petermann, "Normalization of constants in the quanta theory," Helv.Phys.Acta, vol. 26, pp. 499-520, 1953.

[6] A. Peterman, "Renormalization group and the deep structure of the proton," Phys.Rept., vol. 53, p. 157, 1979.

[7] M. Gell-Mann and F. Low, "Quantum electrodynamics at small distances," Phys.Rev., vol. 95, pp. 1300-1312, 1954.

[8] N. Bogolyubov and D. Shirkov, "Charge renormalization group in quantum field theory," Nuovo Cim., vol. 3, pp. 845-863, 1956.

[9] N. Bogoliubov and D. Shirkov, Introduction to the theory of quantized fields. Interscience monographs in physics and astronomy, Interscience Publishers, 1959 .

[10] H. D. Politzer, "Reliable perturbative results for strong interactions?," Phys. Rev.Lett., vol. 30, pp. 1346-1349, 1973.

[11] D. Gross and F. Wilczek, "Ultraviolet behavior of nonabelian gauge theories," Phys.Rev.Lett., vol. 30, pp. 1343-1346, 1973.

[12] J. Callan, Curtis G., "Broken scale invariance in scalar field theory," Phys.Rev.D, vol. 2, pp. 1541-1547, 1970.

[13] K. Symanzik, "Small distance behavior in field theory and power counting," Commun.Math.Phys., vol. 18, pp. 227-246, 1970. 
[14] K. Symanzik, "Small distance behavior analysis and Wilson expansion," Commun.Math.Phys., vol. 23, pp. 49-86, 1971.

[15] N. Bohr and V. WISSKOPF, Niels Bohr and the Development of Physics, Etc. McGraw-Hill Book Company; Pergamon Press: London, 1955.

[16] K. Wilson, "The renormalization group and critical phenomena," Rev. Mod. Phys., vol. 55, pp. 583-600, 1983.

[17] L. Brown, Renormalization: From Lorentz to Landau (And Beyond). Theoretical Physics Series, SPRINGER VERLAG GMBH, 1993.

[18] D. Gross, "Asymptotic freedom and QCD: A historical perspective," Nucl. Phys. Proc.Suppl., vol. 135, pp. 193-211, 2004.

[19] K. G. Wilson and J. Kogut, "The renormalization group and the $\epsilon$ expansion," Phys.Rept., vol. 12, no. 2, pp. 75 - 199, 1974.

[20] K. G. Wilson, "Non-Lagrangian models of current algebra," Phys. Rev., vol. 179, pp. 1499-1512, Mar 1969.

[21] R. H. Swendsen, "Monte Carlo renormalization group," Phys. Rev. Lett., vol. 42, pp. 859-861, Apr 1979.

[22] S. H. Shenker and J. Tobochnik, "Monte Carlo renormalization-group analysis of the classical Heisenberg model in two dimensions," Phys. Rev. B, vol. 22, pp. 4462-4472, Nov 1980.

[23] J. E. Hirsch and S. H. Shenker, "Block-spin renormalization group in the large-n limit," Phys. Rev. B, vol. 27, pp. 1736-1744, Feb 1983.

[24] D. J. E. Callaway and R. Petronzio, "Monte Carlo renormalization group trajectories in lattice gauge theories," Phys. Lett. B, vol. 145, pp. 381-387, Sept. 1984.

[25] A. Hasenfratz, P. Hasenfratz, U. Heller, and F. Karsch, "Improved Monte Carlo renormalization group methods," Phys. Lett. B, vol. 140, pp. 76-82, May 1984.

[26] G. S. Pawley, R. H. Swendsen, D. J. Wallace, and K. G. Wilson, "Monte Carlo renormalization-group calculations of critical behavior in the simplecubic Ising model," Phys. Rev. B, vol. 29, pp. 4030-4040, Apr 1984. 
[27] G. Murthy and R. Shankar, "Possible tests and improvements for Monte Carlo renormalization-group studies," Phys. Rev. Lett., vol. 54, pp. 1110-1113, Mar 1985.

[28] R. Shankar, R. Gupta, and G. Murthy, "Dealing with truncation in Monte Carlo renormalization-group calculations," Phys. Rev. Lett., vol. 55, pp. 18121815, Oct 1985.

[29] M. Falcioni, G. Martinelli, M. Paciello, G. Parisi, and B. Taglienti, "A new proposal for the determination of the renormalization group trajectories by the monte carlo renormalization group method," Nucl. Phys. B, vol. 265, no. 1, pp. $187-196,1986$.

[30] U. M. Heller, "Monte Carlo renormalization-group investigation of the twodimensional O(4) $\sigma$ model," Phys. Rev. Lett., vol. 60, pp. 2235-2237, May 1988.

[31] A. Gonzalez-Arroyo, M. Okawa, and Y. Shimizu, "Monte Carlo renormalizationgroup study of the four-dimensional $\mathrm{Z}_{2}$ gauge theory," Phys. Rev. Lett., vol. 60, pp. 487-490, Feb 1988.

[32] A. Hasenfratz, "The critical behaviour of the four-dimensional U(1) gauge model; An MCRG study," Phys. Lett. B, vol. 201, pp. 492-496, Feb. 1988.

[33] C. F. Baillie, R. Gupta, K. A. Hawick, and G. S. Pawley, "Monte Carlo renormalization-group study of the three-dimensional Ising model," Phys. Rev. B, vol. 45, pp. 10438-10453, May 1992.

[34] P. de Forcrand et al., "Monte Carlo renormalization group analysis of QCD in two-dimensional coupling space," Nucl.Phys.Proc.Suppl., vol. 83, pp. 872-874, 2000 .

[35] E. Tomboulis and A. Velytsky, "RG decimation study of SU(2) gauge theory," PoS, vol. LAT2006, p. 077, 2006.

[36] E. T. Tomboulis and A. Velytsky, "Improved actions and lattice coarsening effects in MCRG studies in SU(2) LGT," ArXiv e-prints, Sept. 2007.

[37] E. T. Tomboulis and A. Velytsky, "Improving the improved action," Phys. Rev. Lett., vol. 98, p. 181601, May 2007.

[38] E. T. Tomboulis and A. Velytsky, "Renormalization group therapy," Phys. Rev. D, vol. 75, p. 076002, Apr 2007. 
[39] E. T. Tomboulis and A. Velytsky, "String tension and removal of lattice coarsening effects in Monte Carlo renormalization group," International Journal of Modern Physics A, vol. 23, pp. 803-812, 2008.

[40] A. Hasenfratz, "Investigating the critical properties of beyond-QCD theories using Monte Carlo renormalization group matching," Phys. Rev. D, vol. 80, p. 034505, Aug 2009.

[41] A. Hasenfratz, "Conformal or walking? Monte Carlo renormalization group studies of $S U(3)$ gauge models with fundamental fermions," Phys. Rev. D, vol. 82, p. 014506, Jul 2010.

[42] A. Hasenfratz, "Scaling properties of many-fermion systems from MCRG studies," ArXiv e-prints, Nov. 2009.

[43] A. Hasenfratz, "Infrared fixed point of the 12-fermion $S U(3)$ gauge model based on 2-Lattice Monte Carlo renomalization-group matching," Phys. Rev. Lett., vol. 108, p. 061601, Feb 2012.

[44] A. Hasenfratz, "MCRG study of 12 fundamental flavors with mixed fundamentaladjoint gauge action," PoS, vol. LATTICE2011, p. 065, 2011.

[45] G. Petropoulos, A. Cheng, A. Hasenfratz, and D. Schaich, "MCRG study of 8 and 12 fundamental flavors," ArXiv e-prints, Nov. 2012.

[46] S.-k. Ma, "Renormalization group by Monte Carlo methods," Phys. Rev. Lett., vol. 37, pp. 461-464, Aug 1976.

[47] F. Sannino, "Conformal dynamics for TeV physics and cosmology," Acta Phys. Polon. B, Nov. 2009.

[48] T. DeGrand, "Lattice studies of QCD-like theories with many fermionic degrees of freedom," ArXiv e-prints, Oct. 2010.

[49] Y. Liu, Y. Meurice, and H. Zou, "Volume effects in discrete $\beta$ functions," PoS, vol. LATTICE2011, p. 246, 2011.

[50] A. Denbleyker, D. Du, Y. Liu, Y. Meurice, and H. Zou, "Fisher's zeros as the boundary of renormalization group flows in complex coupling spaces," Phys. Rev. Lett., vol. 104, p. 251601, June 2010.

[51] A. Bazavov, A. Denbleyker, D. Du, Y. Liu, Y. Meurice, et al., "Fisher's zeros as boundary of RG flows in complex coupling space," PoS, vol. LATTICE2010, p. $259,2010$. 
[52] Y. Liu and Y. Meurice, "Lines of fisher's zeros as separatrices for complex renormalization group flows," Phys. Rev. D, vol. 83, p. 096008, May 2011.

[53] T. L. Curtright and C. K. Zachos, "Renormalization group functional equations," Phys. Rev. D, vol. 83, p. 065019, Mar. 2011.

[54] A. Denbleyker, D. Du, Y. Liu, Y. Meurice, and A. Velytsky, "Series expansions of the density of states in SU(2) lattice gauge theory," Phys. Rev. D, vol. 78, pp. 054503-+, Sept. 2008.

[55] A. Denbleyker, D. Du, Y. Liu, Y. Meurice, and A. Velytsky, "Approximate forms of the density of states," PoS, vol. LATTICE2008, p. 249, 2008.

[56] A. Denbleyker, Y. Liu, Y. Meurice, and A. Velytsky, "Finite size scaling and universality in $S U(2)$ at finite temperature," PoS, vol. LAT2009, p. 197, 2009.

[57] Y. Meurice, A. Bazavov, B. A. Berg, D. Du, A. Denbleyker, et al., "Fisher zeros and conformality in lattice models," PoS, vol. LATTICE2012, p. 229, 2012 .

[58] S. R. White, "Density matrix formulation for quantum renormalization groups," Phys. Rev. Lett., vol. 69, pp. 2863-2866, Nov 1992.

[59] M. Levin and C. P. Nave, "Tensor renormalization group approach to twodimensional classical lattice models," Phys. Rev. Lett., vol. 99, p. 120601, Sep 2007.

[60] Z. Y. Xie, J. Chen, M. P. Qin, J. W. Zhu, L. P. Yang, and T. Xiang, "Coarsegraining renormalization by higher-order singular value decomposition," Phys. Rev. B, vol. 86, p. 045139, Jul 2012.

[61] W. Lenz, "Beitrge zum Verstndnis der magnetischen Eigenschaften in festen Krpern," Physik. Z., vol. 31, pp. 613-615, 1920.

[62] E. Ising, "Beitrag zur Theorie des Ferromagnetismus," Zeitschrift fur Physik, vol. 31, pp. 253-258, Feb. 1925.

[63] R. Peierls and M. Born, "On Ising's model of ferromagnetism," Proceedings of the Cambridge Philosophical Society, vol. 32, p. 477, 1936.

[64] H. A. Kramers and G. H. Wannier, "Statistics of the Two-Dimensional Ferromagnet. Part I," Phys. Rev., vol. 60, pp. 252-262, Aug 1941.

[65] L. Onsager, "Crystal Statistics. I. A Two-Dimensional Model with an OrderDisorder Transition," Phys. Rev., vol. 65, pp. 117-149, Feb 1944. 
[66] J. D. van der Waals, Over de Continuiteit van den Gas- en Vloeistoftoestand (on the continuity of the gas and liquid state). PhD thesis, Leiden, The Netherlands, 1873.

[67] J. Waals and J. Rowlinson, J.D. van der Waals: on the continuity of the gaseous and liquid states. Studies in statistical mechanics, North-Holland, 1988.

[68] J. van der Waals and J. Rowlinson, On the Continuity of the Gaseous and Liquid States. Dover Books on Physics Series, DOVER PUBN Incorporated, 2004.

[69] J. Dillinger, Low Temperature Physics and Chemistry: Proceedings of the Fifth International Conference on Low Temperature Physics and Chemistry, Held at the University of Wisconsin Madison, Wisconsin, August 26-31, 1957. Proceedings of the international conference on low temperature physics, University of Wisconsin Press, 1958.

[70] M. I. Bagatskii, A. V. Voronel', and V. G. Gusak, "Measurement of the specific heat $\mathrm{C}_{v}$ of argon in the immediate vicinity of the critical point," Soviet Physics JETP, vol. 16, p. 517, 1963.

[71] C. N. Yang and T. D. Lee, "Statistical theory of equations of state and phase transitions. I. theory of condensation," Phys. Rev., vol. 87, pp. 404-409, Aug 1952.

[72] T. D. Lee and C. N. Yang, "Statistical theory of equations of state and phase transitions. II. lattice gas and Ising model," Phys. Rev., vol. 87, pp. 410-419, Aug 1952.

[73] W. Brittin, Statistical Physics, Weak Interactions, Field Theory: Lectures Delivered at The Summer Institute for Theoretical Physics, University of Colorado, Boulder, 1964. Lectures delivered at the Summer Institute for Theoretical Physics, University of Colorado, Boulder, 1964 / ed. by Wesley E. Brittin, University of Colorado Press, 1965.

[74] C. Itzykson, R. B. Pearson, and J. B. Zuber, "Distribution of zeros in Ising and gauge models," Nucl. Phys. B, vol. 220, pp. 415-433, Sept. 1983.

[75] N. V. Vdovichenko, "A calculation of the partition function for a plane dipole lattice," Soviet Physics JETP, vol. 20, pp. 477-488, 1965.

[76] H. Stanley, Introduction to Phase Transitions and Critical Phenomena. Oxford Science Publications, Oxford University Press on Demand, 1971. 
[77] R. Pathria, Statistical Mechanics. Elsevier Science, 1996.

[78] R. Creswick, H. Farach, and C. Poole, Introduction to renormalization group methods in physics. A Wiley-Interscience publication, Wiley, 1992.

[79] B. McCoy and T. Wu, The two-dimensional Ising model. Harvard University Press, 1973.

[80] J. Fröhlich and T. Spencer, "The phase transition in the one-dimensional Ising Model with 1/ $\mathrm{r}^{2}$ interaction energy," Comm. Math. Phys., vol. 84, pp. 87-101, Mar. 1982.

[81] F. Dyson, Selected Papers of Freeman Dyson: With Commentary. American Mathematical Society, American Mathematical Society, 1996.

[82] F. J. Dyson, "Existence of a phase-transition in a one-dimensional Ising ferromagnet," Comm. Math. Phys., vol. 12, pp. 91-107, June 1969.

[83] F. J. Dyson, "Non-existence of spontaneous magnetization in a one-dimensional Ising ferromagnet," Comm. Math. Phys., vol. 12, pp. 212-215, Sept. 1969.

[84] F. J. Dyson, "An Ising ferromagnet with discontinuous long-range order," Comm. Math. Phys., vol. 21, pp. 269-283, Dec. 1971.

[85] P. W. Anderson and G. Yuval, "Exact results in the Kondo problem: equivalence to a classical one-dimensional Coulomb gas," Phys. Rev. Lett., vol. 23, pp. 89-92, Jul 1969.

[86] P. W. Anderson, G. Yuval, and D. R. Hamann, "Exact results in the Kondo problem. II. scaling theory, qualitatively correct solution, and some new results on one-dimensional classical statistical models," Phys. Rev. B, vol. 1, pp. 4464-4473, Jun 1970.

[87] P. W. Anderson and G. Yuval, "Some numerical results on the Kondo problem and the inverse square one-dimensional Ising model," Journal of Physics C Solid State Physics, vol. 4, pp. 607-620, Apr. 1971.

[88] D. J. Thouless, "Long-range Order in one-dimensional Ising systems," Phys. Rev., vol. 187, pp. 732-733, Nov 1969.

[89] F. J. Dyson, "Existence of a phase transition in a one-dimensional Ising ferromagnet," Commun. Math. Phys., vol. 12, pp. 91-107, 1969.

[90] G. A. Baker, "Ising model with a scaling interaction," Phys.Rev.B, vol. 5, pp. 2622-2633, 1972. 
[91] K. G. Wilson, "Renormalization group and critical phenomena. 2. Phase space cell analysis of critical behavior," Phys. Rev., vol. B4, pp. 3184-3205, 1971.

[92] P. M. Bleher and Y. G. Sinai, "Investigation of the critical point in models of the type of Dyson's hierarchical models," Commun. Math. Phys., vol. 33, pp. 23-42, 1973.

[93] P. M. Bleher and Y. G. Sinai, "Critical indices for Dyson's asymptoticallyhierarchical models," Comm. Math. Phys., vol. 45, pp. 247-278, Oct. 1975.

[94] H. van Beyeren, G. Gallavotti, and H. Knops, "Conservation laws in the hierarchical model," Physica, vol. 78, pp. 541-548, Dec. 1974.

[95] G. Gallavotti and H. Knops, "The hierarchical model and the renormalization group," Nuovo Cimento Rivista Serie, vol. 5, pp. 341-368, July 1975.

[96] P. Collet and J. P. Eckmann, "The $\epsilon$ expansion for the hierarchical model," Commun. Math. Phys., vol. 55, pp. 67-96, 1977.

[97] P. Collet, J.-P. Eckmann, and B. Hirsbrunner, "A numerical test of Borel summability in the $\epsilon$-expansion of the hierarchical model," Phys. Lett. B, vol. 71, no. 2, pp. $385-386,1977$.

[98] P. Collet and J. Eckmann, eds., A renormalization group analysis of the hierarchical model in statistical mechanics, vol. 74 of Lecture Notes in Physics, Berlin Springer Verlag, 1978.

[99] H. Koch and P. Wittwer, "A nontrivial renormalization group fixed point for the Dyson-Baker hierarchical model," Comm. Math. Phys., vol. 164, pp. 627647, Aug. 1994.

[100] K. Gawedzki and A. Kupiainen, "Continuum limit of the hierarchical O(N) non-linear $\sigma$-model," Comm. Math. Phys., vol. 106, pp. 533-550, Dec. 1986.

[101] G. A. Baker and G. R. Golner, "Critical and tricritical behavior in the hierarchical model," Phys. Rev. B, vol. 16, pp. 2081-2094, Sep 1977.

[102] D. Kim and C. J. Thompson, "Critical properties of Dyson's hierarchical model," Journal of Physics A Mathematical General, vol. 10, pp. 1579-1598, Sept. 1977.

[103] D. Kim and C. J. Thompson, "Critical properties of Dyson's hierarchical model. II. Essential singularities of the borderline Ising case," Journal of Physics A Mathematical General, vol. 11, pp. 375-384, Feb. 1978. 
[104] D. Kim and C. J. Thompson, "Critical properties of Dyson's hierarchical model. III. The n-vector and Heisenberg models," Journal of Physics A Mathematical General, vol. 11, pp. 385-401, Feb. 1978.

[105] M. D. Missarov and P. G. Stepanov, “ $\epsilon$-Expansions in Dyson's Hierarchical Model," Theoretical and Mathematical Physics, vol. 139, pp. 672-678, May 2004.

[106] J. Godina, Y. Meurice, and M. Oktay, "High accuracy calculations of the critical exponents of Dyson's hierarchical model," Phys.Rev., vol. D59, p. 096002, 1999.

[107] P. M. Bleher and J. G. Sinai, "Investigation of the critical point in models of the type of Dyson's hierarchical models," Comm. Math. Phys., vol. 33, pp. 23-42, Mar. 1973.

[108] P. Collet and J.-P. Eckmann, eds., A renormalization group analysis of the Hierarchical model in statistical mechanics, vol. 74 of Lecture Notes in Physics, Berlin Springer Verlag, 1978.

[109] S. Franz, T. Jörg, and G. Parisi, "Overlap interfaces in hierarchical spin-glass models," Journal of Statistical Mechanics: Theory and Experiment, vol. 2, p. 2, Feb. 2009.

[110] M. Castellana and G. Parisi, "Renormalization group computation of the critical exponents of hierarchical spin glasses," Phys. Rev. E, vol. 82, p. 040105, Oct 2010.

[111] K. R. Ito, "Renormalization group flow of two-dimensional hierarchical Heisenberg model of Dyson-Wilson type," Comm. Math. Phys., vol. 139, no. 1, pp. 45-70, 1991.

[112] T. C. Dorlas, "Renormalisation of a hierarchical $\phi_{3}{ }^{4}$ model," Journal of Physics A Mathematical General, vol. 21, pp. 1753-1758, Apr. 1988.

[113] P. M. Bleher and P. Major, "Renormalization of Dyson's hierarchical vector valued $\phi^{4}$ model at low temperatures," Comm. Math. Phys., vol. 95, pp. 487532, Dec. 1984.

[114] P. Collet and J.-P. Eckmann, "The $\epsilon$-expansion for the hierarchical model," Comm. Math. Phys., vol. 55, pp. 67-96, Feb. 1977.

[115] Y. Meurice and G. Ordaz, "The elusive asymptotic behavior of the hightemperature expansion of the hierarchical Ising model," Journal of Statistical Physics, vol. 82, pp. 343-365, Jan. 1996. 
[116] J. B. McGuire, "The spherical hierarchical model," Comm. Math. Phys., vol. 32, pp. 215-230, Sept. 1973.

[117] T. Hara, T. Hattori, and H. Watanabe, "Triviality of hierarchical Ising model in four dimensions," Comm. Math. Phys., vol. 220, pp. 13-40, 2001.

[118] Y. Meurice, "Nonlinear aspects of the renormalization group flows of Dyson's hierarchical model," J. Phys., vol. A40, p. R39, 2007.

[119] C.-N. Yang and T. D. Lee, "Statistical theory of equations of state and phase transitions. I: Theory of condensation," Phys. Rev., vol. 87, pp. 404-409, 1952.

[120] T. D. Lee and C.-N. Yang, "Statistical theory of equations of state and phase transitions. II: Lattice gas and Ising model," Phys. Rev., vol. 87, pp. 410-419, 1952.

[121] M. Fisher, in Lectures in Theoretical Physics Vol. VIIC. Boulder, Colorado: University of Colorado Press, 1965.

[122] B. Levin, Distribution of Zeros of Entire Functions. Translations of Mathematical Monographs, American Mathematical Society, 1980.

[123] A. Denbleyker, D. Du, Y. Liu, Y. Meurice, and H. Zou, "Fisher's zeros as boundary of renormalization group flows in complex coupling spaces," Phys. Rev. Lett., vol. 104, p. 251601, 2010.

[124] M. N. Barber in Phase Transitions and Critical Phenomena (C. Domb and J. L. Lebowitz, eds.), vol. 8, London: Academic Press, 1983.

[125] J. L. Cardy in Finite Size Scaling (J. L. Cardy, ed.), vol. 2, North-Holland, 1988.

[126] J. J. Godina, Y. Meurice, and M. B. Oktay, "Accurate checks of universality for Dyson's hierarchical model," Phys. Rev. D, vol. 57, pp. R6581-R6584, Jun 1998.

[127] J. J. Godina, Y. Meurice, and M. B. Oktay, "High-accuracy calculations of the critical exponents of Dyson's hierarchical model," Phys. Rev. D, vol. 59, p. 096002, Apr 1999.

[128] W. Janke, D. A. Johnston, and R. Kenna, "Phase transition strength through densities of general distributions of zeroes," Nucl. Phys. B, vol. 682, pp. 618634, Mar. 2004. 
[129] C. Bagnuls and C. Bervillier, "Exact renormalization group equations. An Introductory review," Phys.Rept., vol. 348, p. 91, 2001.

[130] J. Berges, N. Tetradis, and C. Wetterich, "Non-perturbative renormalization flow in quantum field theory and statistical physics," Phys. Rept., vol. 363, pp. 223-386, 2002.

[131] J. Polchinski, "Renormalization and effective Lagrangians," Nucl. Phys. B, vol. 231, pp. 269-295, 1984.

[132] A. Hasenfratz and P. Hasenfratz, "Renormalization group study of scalar field theories," Nucl. Phys., vol. B270, pp. 687-701, 1986.

[133] G. Felder, "Renormalization group in the local potential approximation," Comm. Math. Phys., vol. 111, pp. 101-121, Mar. 1987.

[134] J. Gottker-Schnetmann, "O(N)-invariant hierarchical renormalization group fixed points by algebraic numerical computation and epsilon expansion," 1999.

[135] D. F. Litim, "Optimisation of the exact renormalisation group," Phys. Lett., vol. B486, pp. 92-99, 2000.

[136] M. B. Oktay, "Nonperturbative methods for hierarchical models," 2001. (Ph. D. thesis), UMI-30-18602.

[137] J. J. Godina, L. Li, Y. Meurice, and M. B. Oktay, "High-accuracy critical exponents of $\mathrm{O}(\mathrm{N})$ hierarchical sigma models," Phys. Rev., vol. D73, p. 047701, 2006.

[138] C. Bervillier, A. Juttner, and D. F. Litim, "High-accuracy scaling exponents in the local potential approximation," 2007.

[139] G. Gallavotti, P. Zweifel, and N. A. T. O. S. A. Division, Nonlinear evolution and chaotic phenomena. NATO ASI series: Physics, Plenum Press, 1988.

[140] D. F. Litim, "Critical exponents from optimised renormalisation group flows," Nucl. Phys., vol. B631, pp. 128-158, 2002.

[141] A. Hasenfratz, "Investigating the critical properties of beyond-qcd theories using monte carlo renormalization group matching," Phys. Rev. D, vol. 80, p. 034505, Aug 2009.

[142] J. Pool, A. M. Society, S. for Industrial, and A. Mathematics, Mathematical Aspects of Statistical Mechanics. SIAM-AMS Proceedings, American Mathematical Society, 1972. 
[143] S. Moroz and R. Schmidt, "Nonrelativistic inverse square potential, scale anomaly, and complex extension," Ann. Phys., vol. 325, pp. 491-513, Feb. 2010 .

[144] K. Wilson and J. B. Kogut, "The renormalization group and the epsilon expansion," Phys.Rept., vol. 12, pp. 75-200, 1974.

[145] F. J. Wegner and A. Houghton, "Renormalization group equation for critical phenomena," Phys.Rev., vol. A8, pp. 401-412, 1973.

[146] J. Berges, N. Tetradis, and C. Wetterich, "Nonperturbative renormalization flow in quantum field theory and statistical physics," Phys.Rept., vol. 363, pp. 223-386, 2002.

[147] D. Jungnickel and C. Wetterich, "Flow equations for phase transitions in statistical physics and QCD," pp. 41-113, 1998.

[148] D. Jungnickel and C. Wetterich, "Nonperturbative flow equations in QCD," Prog.Theor.Phys.Suppl., vol. 131, pp. 495-549, 1998.

[149] Y. Ivanchenko and A. Lisyansky, Physics of Critical Fluctuations. Graduate Texts in Contemporary Physics, Springer, 1995.

[150] K. Huang, Quantum Field Theory. Wiley, 2008.

[151] P. Kopietz, L. Bartosch, and F. Schütz, Introduction to the Functional Renormalization Group. Lecture Notes in Physics, Springer, 2010.

[152] D. F. Litim, "Mind the gap," Int.J.Mod.Phys., vol. A16, pp. 2081-2088, 2001.

[153] D. F. Litim, "Universality and the renormalisation group," JHEP, vol. 7, p. 5, July 2005.

[154] T. R. Morris, "Equivalence of local potential approximations," JHEP, vol. 7, p. 27, July 2005.

[155] C. Bervillier, A. Jüttner, and D. F. Litim, "High-accuracy scaling exponents in the local potential approximation," Nucl. Phys. B, vol. 783, pp. 213-226, Nov. 2007.

[156] M. Bonini, M. D’Attanasio, and G. Marchesini, "Perturbative renormalization and infrared finiteness in the Wilson renormalization group: The Massless scalar case," Nucl.Phys., vol. B409, pp. 441-464, 1993. 
[157] C. Wetterich, "Exact evolution equation for the effective potential," Phys. Lett. B, vol. 301, pp. 90-94, 1993.

[158] U. Ellwanger, "FLow equations for N point functions and bound states," Z.Phys., vol. C62, pp. 503-510, 1994.

[159] T. R. Morris, "The exact renormalization group and approximate solutions," Int.J.Mod.Phys., vol. A9, pp. 2411-2450, 1994.

[160] G. R. Golner, "Nonperturbative renormalization-group calculations for continuum spin systems," Phys. Rev. B, vol. 33, pp. 7863-7866, Jun 1986.

[161] K. G. Wilson and M. E. Fisher, "Critical exponents in 3.99 dimensions," Phys. Rev. Lett., vol. 28, pp. 240-243, Jan 1972.

[162] K. G. Wilson, "Renormalization group and critical phenomena. II. phasespace cell analysis of critical behavior," Phys. Rev. B, vol. 4, pp. 3184-3205, Nov 1971.

[163] N. Tetradis and C. Wetterich, "Critical exponents from effective average action," Nucl.Phys., vol. B422, pp. 541-592, 1994.

[164] P. M. Stevenson, "Optimized perturbation theory," Phys. Rev. D, vol. 23, pp. 2916-2944, Jun 1981.

[165] D. F. Litim, "Optimization of the exact renormalization group," Phys.Lett., vol. B486, pp. 92-99, 2000.

[166] D. F. Litim, "Towards functional flows for hierarchical models," Phys. Rev. D, vol. 76, p. 105001, 2007.

[167] T. R. Morris, "On truncations of the exact renormalization group," Phys. Lett. B, vol. 334, pp. 355-362, Aug. 1994.

[168] K. Aoki, K. Morikawa, W. Souma, J. Sumi, and H. Terao, "The effectiveness of the local potential approximation in the Wegner-Houghton renormalization group," Progress of Theoretical Physics, vol. 95, pp. 409-420, Feb. 1996.

[169] K. Aoki, K. Morikawa, W. Souma, J. Sumi, and H. Terao, "Rapidly converging truncation scheme of the exact renormalization group," Progress of Theoretical Physics, vol. 99, pp. 451-466, Mar. 1998.

[170] D. F. Litim, "Critical exponents from optimised renormalisation group flows," Nucl. Phys. B, vol. 631, pp. 128-158, June 2002. 
[171] B. Svetitsky and L. G. Yaffe, "Critical behavior at finite-temperature confinement transitions," Nucl. Phys. B, vol. 210, pp. 423-447, Dec. 1982.

[172] J. Engels, J. Fingberg, and M. Weber, "Finite size scaling analysis of $S U(2)$ lattice gauge theory in (3+1)-dimensions," Nucl. Phys., vol. B332, p. 737, 1990.

[173] J. Fingberg, U. M. Heller, and F. Karsch, "Scaling and asymptotic scaling in the SU(2) gauge theory," Nucl. Phys., vol. B392, pp. 493-517, 1993.

[174] I. L. Bogolubsky, V. K. Mitrjushkin, M. Müller-Preussker, A. V. Sergeev, and H. Stüben, "Polyakov loops and binder cumulants in SU(2) theory on large lattices," Nuclear Physics B Proceedings Supplements, vol. 129, pp. 611-613, Mar. 2004.

[175] A. Papa and C. Vena, "Finite-size scaling and deconfinement transition:," International Journal of Modern Physics A, vol. 19, pp. 3209-3216, 2004.

[176] A. Velytsky, "Finite temperature $S U(2)$ gauge theory:. critical coupling and universality class," International Journal of Modern Physics C, vol. 19, pp. 10791093, 2008.

[177] J. Deckert, S. Wansleben, and J. G. Zabolitzky, "Monte Carlo calculation for the effective couplings of Polyakov loops," Phys. Rev. D, vol. 35, pp. 683-690, Jan 1987.

[178] S. Wansleben, "On the universality class of the deconfinement transition in lattice gauge theories at finite temperature," Phys. Lett. B, vol. 148, no. 6, pp. $461-464,1984$.

[179] K. G. Wilson, "Confinement of quarks," Phys. Rev. D, vol. 10, pp. 2445-2459, Oct 1974.

[180] M. Hasenbusch, K. Pinn, and S. Vinti, "Critical exponents of the threedimensional Ising universality class from finite-size scaling with standard and improved actions," Phys. Rev. B, vol. 59, pp. 11471-11483, May 1999.

[181] Y. Meurice, "How to control nonlinear effects in Binder cumulants," ArXiv e-prints, Dec. 2007.

[182] K. Binder, "Critical properties from monte carlo coarse graining and renormalization," Phys. Rev. Lett., vol. 47, pp. 693-696, Aug 1981.

[183] A. M. Ferrenberg and D. P. Landau, "Critical behavior of the three-dimensional Ising model: A high-resolution Monte Carlo study," Phys. Rev. B, vol. 44, 
pp. 5081-5091, Sep 1991.

[184] M. Campostrini, A. Pelissetto, P. Rossi, and E. Vicari, "Two-point correlation function of three-dimensional $O(N)$ models: The critical limit and anisotropy," Phys. Rev. E, vol. 57, pp. 184-210, Jan 1998.

[185] M. A. Clark and A. D. Kennedy, "Accelerating staggered-fermion dynamics with the rational hybrid Monte Carlo algorithm," Phys. Rev. D, vol. 75, p. 011502, Jan 2007.

[186] M. A. Clark and A. D. Kennedy, "Accelerating dynamical-fermion computations using the Rational Hybrid Monte Carlo algorithm with multiple pseudofermion fields," Phys. Rev. Lett., vol. 98, p. 051601, Jan 2007.

[187] M. A. Clark and A. D. Kennedy, "The RHMC algorithm code," 2005. 